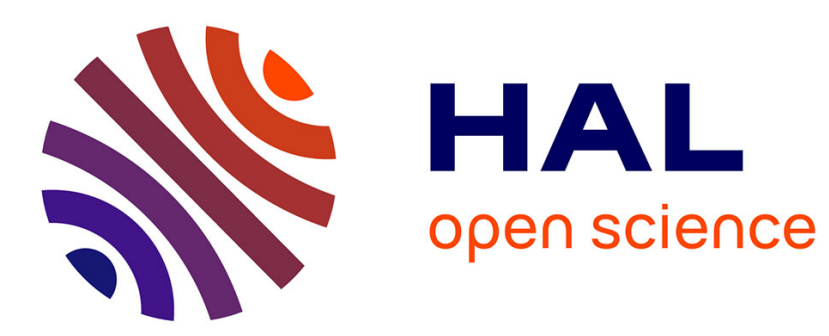

\title{
Detailed chemical kinetic models for the low-temperature combustion of hydrocarbons with application to gasoline and diesel fuel surrogates
}

Frédérique Battin-Leclerc

\section{- To cite this version:}

Frédérique Battin-Leclerc. Detailed chemical kinetic models for the low-temperature combustion of hydrocarbons with application to gasoline and diesel fuel surrogates. Progress in Energy and Combustion Science, 2008, 34, pp.440-498. 10.1016/j.pecs.2007.10.002 . hal-00288708

\section{HAL Id: hal-00288708 \\ https://hal.science/hal-00288708}

Submitted on 18 Jun 2008

HAL is a multi-disciplinary open access archive for the deposit and dissemination of scientific research documents, whether they are published or not. The documents may come from teaching and research institutions in France or abroad, or from public or private research centers.
L'archive ouverte pluridisciplinaire HAL, est destinée au dépôt et à la diffusion de documents scientifiques de niveau recherche, publiés ou non, émanant des établissements d'enseignement et de recherche français ou étrangers, des laboratoires publics ou privés. 
DETAILED CHEMICAL KINETIC MODELS FOR THE LOW TEMPERATURE

COMBUSTION OF HYDROCARBONS WITH APPLICATION TO

GASOLINE AND DIESEL FUEL SURROGATES

F.BATTIN-LECLERC

Département de Chimie-Physique des Réactions, UMR n7630 CNRS, INPL-ENSIC,

1 rue Grandville, BP 20451, 54001 NANCY Cedex, France

Tél. 33(0)3.83.17.51.25 - Fax 33(0)3.83.37.81.20

e-mail : Frederique.Battin-Leclerc@ensic.inpl-nancy.fr

Full-length review article 


\section{ABSTRACT}

This paper presents a review of gas-phase detailed kinetic models developed to simulate the low temperature oxidation and autoignition of gasoline and diesel fuel components (alkanes, ethers, esters, alkenes, cycloalkanes, aromatics, including from 4 atoms of carbon) and of mixtures of several of them, which have been proposed as surrogates. The recently proposed models are summarized, as well as the experimental results available for their validation. A comparison between the major models in terms of considered elementary steps and associated rate constants is also proposed.

Keywords: [Detailed kinetic model], [combustion], [autoignition], [low temperature oxidation], [gasoline], [diesel fuel], [bio-fuel], [surrogate]. 


\section{NOMENCLATURE}

CV: closed vessel

DIPE: di-isopropyl-ether

ETBE: ethyl-tert-butyl-ether

FR: flow reactor

HCCI: homogeneous charge compression ignition

JSR: jet stirred reactor

$\mathrm{k}$ : $\quad$ rate constant, $\mathrm{k}=\mathrm{A} \mathrm{T}^{\mathrm{n}} \exp (-\mathrm{Ea} / \mathrm{RT})$

MON: motor octane number

MTBE: methyl-tert-butyl-ether

NTC: negative temperature coefficient

P: $\quad$ pressure

$\mathrm{P}_{\mathrm{c}}$ : $\quad$ pressure after compression in a $\mathrm{RCM}$

PRF: primary reference fuels, i.e. n-heptane and iso-octane (1,2,4-trimethylpentane)
R: $\quad$ gas constant

RCM: rapid compression machine

RON: research octane number

ST: $\quad$ shock tube

$\mathrm{T}: \quad$ temperature

$\mathrm{T}_{\mathrm{c}}$ : temperature after compression in a rapid compression machine

TAME:tert-amyl-methyl-ether

$\phi: \quad$ equivalence ratio, i.e. for the reaction

$\mathrm{C}_{\mathrm{n}} \mathrm{H}_{\mathrm{m}}+(\mathrm{n}+\mathrm{m} / 4) \mathrm{O}_{2} \rightarrow \mathrm{n} \mathrm{CO} \mathrm{CO}_{2}+\mathrm{m} / 2 \mathrm{H}_{2} \mathrm{O}$

$\phi=\frac{\left(\frac{\% \mathrm{C}_{\mathrm{n}} \mathrm{H}_{\mathrm{m}}}{\% \mathrm{O}_{2}}\right)}{\left(\frac{\% \mathrm{C}_{\mathrm{n}} \mathrm{H}_{\mathrm{m}}}{\% \mathrm{O}_{2}}\right)_{\text {stoichiométric }}}=\frac{\left(\frac{\% \mathrm{C}_{\mathrm{n}} \mathrm{H}_{\mathrm{m}}}{\% \mathrm{O}_{2}}\right)}{\frac{1}{\mathrm{n}+\mathrm{m} / 4}}$

$\tau: \quad$ residence time (space time) in a JSR 


\section{CONTENT LIST}

1/ INTRODUCTION

2/ ALKANES, ETHERS AND (METHYL AND ETHYL) ESTERS __ 10

2.1/ Main chemical features of their oxidation__ 10

2.2/ Detailed chemical models of low temperature oxidation __ 13

2.2.a/ Mechanisms written without computer help ___ 13

2.2.b/ Computer aided mechanism generation $\longrightarrow 15$

2.3/ Experimental results available for validation 18

2.4/ Comparison between the major models in terms of elementary steps and associated rate constants ___ 25

2.4.a/ Reactions of alkyl radicals $(\mathrm{R} \bullet)$

2.4.b/ Reactions of peroxyalkyl radicals (ROO•)

$2.4 . c /$ Reactions of hydroperoxyalkyl radicals $(\bullet \overline{\mathrm{QOOH}})$

2.4.d/ Reactions of peroxyhydroperoxyalkyl radicals $(\cdot \mathrm{OOQOOH})[33$

2.4.e/ Secondary reactions of hydroperoxides ___ 34

2.4.f/ Secondary reactions of cyclic ethers — 35

2.4.g/ Classes of reactions and kinetic data specific to the oxidation of acyclic ethers 36

2.4.h/ Classes of reactions and kinetic data specific to the oxidation of methyl and ethyl esters __ 36

2.5/ Conclusion on the modelling of the oxidation of alkanes, ethers and methyl and ethyl esters ___ 37

3/ ALKENES _ 38

3.1/ Main chemical features of their oxidation___ 39

3.2/ Low temperature oxidation models and experimental results available for their validation ___ 41

3.4/ Elementary steps and associated rate constants specific to the reactions of alkenes

3.4.a/ Additions to the double bond___ 44

3.4.b/ Reactions of alkenyl and hydroxyalkyl radicals __ 45

3.4.c/ Reactions of peroxyradicals___ 46

3.4.d/ Reactions of hydroperoxyradicals ___ 47

3.4.e/ Secondary reactions ___ 48

$3.5 /$ Conclusion on the modelling of the oxidation of alkenes ___ 48

4/ CYCLOALKANES _ 49

4.1/ Main chemical features of their oxidation___ 49

4.2/ Low temperature oxidation models and experimental results available for their validation ___ 51

4.3/ Comparison between the major models in terms of elementary steps and associated rate constants ___ 53

4.3.a/ Reactions of cyclo-alkylperoxy radicals __ 53

4.3.b/ Reactions of cyclo-hydroperoxyalkyl radicals _ـ 55

4.3.c/ Reactions of cyclo-hydroperoxyalkylperoxy radicals ___56

4.4/ Conclusion on the modelling of the oxidation of cycloalkanes_ 57

5/ AROMATIC COMPOUNDS _ 57

5.1/ Main chemical features of their oxidation___ 58

5.2 / Detailed chemical models of oxidation ___ 60

5.3/ Experimental results available for validation ___ 63

5.4/ Comparison between the major models in terms of elementary steps and associated rate constants ___ 66

5.4.a/ Additions to the aromatic cycles and derived reactions $\quad-66$

5.4.b/ Reactions of phenyl radicals __ 68 
5.4.c/ Reactions of benzyl radicals 69

5.4.d/ Reactions of cyclopentadienyl radicals 72

5.5/ Conclusion on the modelling of the oxidation of aromatic compounds 73

6/ APPLICATIONS TO SURROGATE MIXTURES ___ 74

6.1/ Main features about reaction coupling __ 75

6.2/ Detailed chemical models of the low temperature oxidation __ 76

6.3/ Experimental results available for validation ___ 81

6.4/ Comparison between the major models in terms of elementary steps and associated rate constants ___ 83 6.4.a/ Mixtures of alkanes $\quad-83$

6.4.b/Alkane/alkene blends___ 84

6.4.c/ Mixtures containing an aromatic compound ___ 85

$6.5 /$ Conclusion on the modelling of the oxidation of mixtures ___ 86

8/ CONCLUSION___ 87

ACKNOWLEDGEMENTS _ 94

REFERENCES _ 


\section{1/ INTRODUCTION}

As reviewed by Westbrook et al. [1] computer simulation of a wide range of phenomena in internal combustion engines has been a particularly fertile research area over the past 30 years. From the chemical point of view, the understanding of knock phenomena and the search for anti-knock additives for fuels used in spark ignition engines [2-4], the modelling of the formation of soot in diesel engines [5-6], the recent development of HCCI (Homogeneous Charge Compression Ignition) engines [7] and the reduction of the emission of toxic gas or pollutants $(\mathrm{CO}, \mathrm{NOx}$, aldehydes, dienes...) in every type of engine have led to an increasing need for the development of kinetic models. More and more sophisticated detailed chemical kinetic models, based on a large number of elementary reactions, have thus been proposed and for a large and widening variety of model fuels.

The need to develop more efficient, but cleaner, engines and fuels is of increasing importance for the automotive and oil industries. Car usage has a significant impact on climate change, with about $12 \%$ of the overall EU emissions of carbon dioxide (CO2), the main greenhouse gas, coming from the fuel consumed by passenger cars [8]. Regulations have been proposed to reduce the emission of this gas from the fuel consumed by combustion engine vehicles. In Europe, the target to be achieved by 2012 is $120 \mathrm{~g} \mathrm{CO}_{2} / \mathrm{km}$ for the average new car fleet [8]. The expected dramatic world-wide increase in the number of combustion engine vehicles (compared to 2000, the number of vehicles could more than double by 2050 [9]) is another reason to promote energy conservation efforts. However a shortage of oil is not foreseen within the next two or three decades, because new oil sources continue to be found and the emergence of better oil field technology enables more oil to be recovered from existing oil wells [9].

Figure 1 illustrates well the importance of tailored detailed kinetics to model the 
combustion in HCCI engines. Pressure curves have been computed by 3D simulations coupled with detailed kinetic models by means of look-up tables for diesel and HCCI engines using two model fuels of n-heptane and a n-decane/ $\alpha$-methylnaphthalene mixture [10]. In the case of diesel engine, only small differences were observed in the autoignition and remaining combustion processes between both model fuels, while in the case of HCCI engine, the same autoignition delay is predicted with both model fuels, but the pressure rise after ignition is slower for the mixture indicating a lower heat release rate.

\section{FIGURE 1}

While gasoline and diesel fuel have a "near-continuous spectrum" of hydrocarbon constituents, surrogates composed of a limited number of components have to be defined in order to develop detailed kinetic models. The limitation in the number of components in surrogates is mainly due to the present availability of detailed kinetic models, but should further persist in order to avoid an explosion of the size of the models. This limitation prevents surrogates reproducing the "near-continuous spectrum" of components in fuels and could induce misleading or inaccurate predicted results. Table 1 presents the composition of a typical European gasoline and shows that its constituents can be divided in six families, each having a carbon number ranging mainly from 4 to 10; they are linear alkanes (n-paraffins), branched alkanes (iso-paraffins), ethers, cyclic alkanes (naphtenes), alkenes (olefins) and aromatic compounds. In the USA, gasolines contain more branched alkanes $(\sim 45 \%)$ and ethers $(\sim 11 \%)$, but less alkenes $(\sim 5 \%)$ and aromatic compounds $(\sim 30 \%)$ [11]. An example of typical diesel fuel is composed of $30.9 \%$ paraffins, $23.7 \%$ alkylcyclohexanes, $15.1 \%$ alkyldecalines, $9.2 \%$ alkylbenzenes and $19.25 \%$ polycyclic naphtenoaromatic compounds, with constituents containing from 10 to 20 atoms of carbon [11].

\section{TABLE 1}


Environmental concerns have led to the increased interest in the future use of fuels, containing a larger fraction of components which have been derived from biomass, such as alcohols (mainly ethanol) in gasoline and methyl or ethyl esters in biodiesel [12-13]. It should be mentioned that the presence of additional oxygenated compounds could reduce the formation of soot in diesel engines [14], but may also promote the formation of some toxic pollutants, such as aldehydes [15-16]. While full life-cycle analyses in order to quantify the extent to which liquid biofuels reduce the impact on global climate are yet to be made [17], methyl and ethyl esters is the seventh family of fuel components, which needs to be taken into account in detailed kinetic models.

The purpose of this review is to analyse the gas-phase detailed kinetic models which have been recently developed to model the oxidation and autoignition of the components of the seven families and of mixtures of several of them, which have been proposed as surrogates. In order to address compounds which are actually representative of those included in gasoline and diesel fuel, only those containing more than four atoms of carbon will be considered. Furthermore, in order to study conditions which are close to those observed in engines, only models which have been tailored to reproduce phenomena occurring at relatively low temperature, i.e. below 900$1000 \mathrm{~K}$, will be reviewed. A more comprehensive and well documented review of detailed chemical kinetic models for the intermediate to high-temperature oxidation, ignition and combustion of alkanes, cycloalkanes, alkenes and aromatic compounds has been recently published by Simmie [18].

The chemistry of nitrogen containing compounds is out of the scope of this review and the kinetic models considering the effect of NOx on combustion [19-22] will not be considered here, despite their interest for the development of HCCI engines by using exhaust gas recirculation (EGR). 
As the chemistry of the oxidation of ethers and esters is usually treated using rules very close to those considered for alkanes, this review contains only five sections, (linear and branched) alkanes, ethers and (methyl and ethyl) esters, alkenes, cycloalkanes, aromatic compounds and application to surrogate mixtures. Each section is structured with the following, when relevant:

- A summary of the main chemical features concerning the oxidation of these compounds.

- A short description of the low temperature models recently proposed, as well as the experimental results available for their validation; only experimental data obtained in a system which can be modelled with a simple physical model and post 1993 studies will be taken into account. Previous models and experimental data have been comprehensively reviewed by Griffiths in 1995 [23].

- A comparison between the major models in terms of considered elementary steps and associated rate constants; only the steps important at low temperature will be reviewed.

The operating conditions for validating a model depend on the sought objectives of the application and the quality of this validation is a function of the amount of experimental data available for comparison with simulations. The comparison between low temperature mechanisms is made possible by the fact that they are based on a limited number of classes of elementary steps. The number of available low temperature mechanisms is also much smaller than that of high temperature mechanisms due to the need to consider many more species and reactions at low temperature. Table 2 presents a comparison between the sizes of low and high temperature mechanisms of several alkanes $[24]^{1}$. It shows that this difference increases strongly with the number of atoms of carbon included in the reactant.

\footnotetext{
${ }^{1}$ Only addresses of web sites from which models, thermochemical data or software can be obtained are given in references.
} 
TABLE 2

Despite being of great importance to ensure the consistency between the rate parameters of a direct and reverse elementary reaction and to estimate the heat release rate, the thermodynamic properties, enthalpies of formation, entropies and heat capacities, used by the different models will not be reviewed here. In most models, the thermodynamic properties for all the species, for which there were no available data in the literature (e.g. [25-26]), were calculated by softwares such as THERM [27] or THERGAS [28] using group additivity rules developed by Benson [29].

The models able to simulate the low temperature oxidation phenomena consider also the typical classes of reactions of high temperature mechanisms [30]. As it is difficult to define classes of elementary steps for reactions involving very small or multi-unsaturated compounds, most detailed mechanisms for the oxidation of hydrocarbons containing more than four atoms of carbon also include a sub-mechanism containing the reactions of smaller species. The development of detailed kinetic models for species representative of components of gasoline and diesel fuel has thus been made possible by the knowledge base which has been previously gained concerning the reactions of light hydrocarbons. Particularly valuable work has been undertaken for two decades by Tsang [31-35] and by Baulch et al. [36-38], who have compiled and evaluated the rate constants of elementary steps important to the oxidation of species containing up to 4 atoms of carbon. The rate constants of the reactions of small species often depend more greatly on pressure and the formalism proposed by Troe [39] has allowed this pressure dependence to be easily taken into account in models. Nevertheless, as reviewed by Simmie [18], the major models [40-43] developed to reproduce the oxidation of methane have been validated against a large spectrum of experiments (species profiles and ignition delay times in shock waves, laminar flame species profiles, laminar flame speeds, temperature and stable species 
concentration profiles in flow reactors), but only at temperatures above $900 \mathrm{~K}$ and large uncertainties still remain on the reactions and rate constants which are used at lower temperature.

The development of the software library of CHEMKIN [44], which can be used to model a wide range of reactors, has also contributed to the increased development of kinetic models. The CHEMKIN input data format has become a standard for describing the reactions, rate parameters, thermodynamic data and transport properties of species, and so this has favoured the exchange of models between kineticists.

\section{2/ ALKANES, ETHERS AND (METHYL AND ETHYL) ESTERS}

As noticed by Simmie [18], alkanes are by far the best studied class of compounds for which reliable and detailed chemical kinetic models for combustion exist. That explains why the search for correlations between fuel chemical structure and octane number rating in spark-ignition engines has mostly been done for alkanes and why n-heptane was often considered as a serviceable surrogate of diesel fuel [1].

\section{1/ Main chemical features of their oxidation}

The first low temperature reaction channels for the oxidation of alkanes were proposed by Knox in 1967 [45] and by Fish in 1968 [46]. The understanding of this mechanism was then improved successively by Pollard [47], Cox and Cole [48] and Walker and Morley [49]. Figure 2 shows a simplified scheme of the main reactions, which are now usually admitted to model the oxidation of an alkane (RH). Except at very high temperature (above $1200 \mathrm{~K}$ ), the reaction is initiated by the $\mathrm{H}$-abstraction from the alkane by oxygen molecules to give alkyl $(\cdot \mathrm{R})$ and hydroperoxy $(\cdot \mathrm{OOH})$ radicals. At low temperature (around 500-600 K), alkyl radicals react rapidly with oxygen molecules to give peroxyalkyl radicals (ROO•) which can by several reactions as shown in figure 2 , lead to the formation of peroxide species and small radicals which 
react with alkane molecules by metatheses to regenerate alkyl radicals. The propagation of the reaction is a chain reaction, in which hydroxyl radicals $(\bullet \mathrm{OH})$ are the main chain carriers.

\section{FIGURE 2}

The formation of peroxides is extremely important, because they include an $\mathrm{O}-\mathrm{OH}$ bond, which can easily be broken and lead to the formation of two radicals, which can in their turn react with alkane molecules to give alkyl radicals. These degenerate branching steps involve a multiplication of the numbers of radicals, which in a chain reaction induces an exponential acceleration of reaction rates leading in some conditions to spontaneous autoignition. The reversibility of the addition of alkyl radicals to oxygen molecules (reaction (1) in figure 2), when the temperature increases to the benefit of the formation of alkenes (reaction (2)), leads to a reduction of the overall reaction rate and is the main cause of the appearance of the negative temperature coefficient (NTC) regime. This distinctive feature of the oxidation of hydrocarbons signifies a zone of temperature in which the global rate of the reaction decreases with temperature.

The existence of the NTC zone explains another specificity of the oxidation of alkanes the possible occurrence of cool flame phenomenon at temperatures several hundred degrees below the minimum autoignition temperature. During a cool flame, or multiple cool flames, the temperature and the pressure increase strongly over a limited temperature range (typically up to $500 \mathrm{~K})$, but the reaction stops before combustion is complete due to the decrease in reactivity in the NTC zone. Cool flames play an important part in spontaneous phenomena as they are the first stage of two stage ignition [50].

The competition between the channels leading to peroxide species and those producing less reactive products, such as alkenes or cyclic ethers, accounts for (at least in part) why the reactivity of alkanes decreases when the level of branching of the molecule increases and 
increases when the length of the included linear chain increases, as shown by the octane numbers $(\mathrm{RON} \text { and } \mathrm{MON})^{1}$ given in Table 2 for some alkanes.

With further increase in temperature, other reactions (such as $\mathrm{H}_{2} \mathrm{O}_{2} \rightarrow 2 \mathrm{OH} \bullet$ and $\mathrm{H} \bullet+\mathrm{O}_{2}$ $\rightarrow \bullet \mathrm{OH}+\bullet \mathrm{O} \bullet$ ) ensure the multiplication of the number of radicals and are responsible for the propagation of combustion in spark ignited engines and for autoignition in diesel engines. Above 900-1000 K, reaction (3), the decomposition to give a smaller alkyl radical and a 1-alkene molecule is the preponderant fate of most alkyl radicals containing more than three atoms of carbon. H-abstractions followed by isomerizations and successive decompositions of alkyl radicals until the relatively well-known chemistry of $\mathrm{C}_{1}-\mathrm{C}_{2}$ species [18] is reached, constitute the high temperature mechanism as first proposed by Westbrook and Dryer [51] and by Warnatz [52].

Due to their low reactivity (RON usually above 100 [11]), $\mathrm{C}_{5}-\mathrm{C}_{6}$ ethers (e.g. methyl tertbutyl ether (MTBE), tert-amyl-methyl ether (1,1-Dimethylpropyl methyl ether, TAME), ethyl tert-butyl ether (ETBE) or di-isopropyl-ether (DIPE)) have been proposed as octane improvers in gasolines. The chemistry of the oxidation of saturated branched ethers seems to be very close to that of alkanes, as shown by the mechanistic studies of the oxidation of MTBE, ETBE and TAME made by the team of Baronnet [53-54] and the modelling study of the oxidation of dimethyl ether (DME) made by the team of Dryer [55-56]. The only specificity of ethers is a molecular reaction involving the transfer of a $\mathrm{H}$-atom bound to an atom of carbon in $\beta$ position of the atom of oxygen to give an alcohol molecule and an alkyl radical as proposed by Choo et al. [57]. This molecular reaction was proven to be very sensitive to wall effects [58]. The presence of an atom of oxygen also favors the decomposition of radicals derived from ethers,

\footnotetext{
1 Research Octane Number (RON) or Motor Octane Number (MON) are octane ratings depending on the used experimental method and measure of how resistant gasoline is to auto-ignition (knocking).
} 
which occurs at a lower temperature than in the case of alkanes. This easier decomposition, as well as the molecular reaction, produces alkenes, such as iso-butene, that have a strong inhibiting effect, lowering the possibility of formation of hydroperoxide species and which explains the lower reactivity of these compounds [53].

Fuels derived from renewable agricultural fats and oils have the potential to supplant a fraction of petroleum products in diesel engines combustion [13]. Vegetal oils mainly include triglycerides (98\%), with small amounts of mono and diglycerides. The fatty acid composition of rapeseed oil, which is of importance in Europe, is $64.4 \%$ of oleic (C18:1 monounsaturated), 22.3\% linoleic (C18:2 omega-6 polyunsaturated), 8.2\% alpha-linolenic (C18:3 omega-3 polyunsaturated), 3.5\% palmitic (C16:0 saturated) and 0.9\% stearic (C18:0 saturated) acids [59]. Methyl or ethyl esters can be obtained from vegetal oils through a catalytic transesterification reaction with methanol or ethanol, respectively [13]. Comparing results obtained in static reactors at subatmospheric pressures by previous authors, Baronnet and Brocard [60] have noticed that the cool flame behaviour of $\mathrm{C}_{2}-\mathrm{C}_{5}$ esters strongly depends on their structure. While they are observed for all ethyl esters, cool flames can only be obtained for methyl esters containing at least five atoms of carbon. More recently, Dagaut et al. [61] have shown that nhexadecane could be a good surrogate model fuel for the oxidation of rapeseed oil methyl ester in a jet-stirred reactor at temperatures between 900 and $1200 \mathrm{~K}$, atmospheric pressure and equivalence ratios from 0.25 to 1.5 .

\section{2/ Detailed chemical models of low temperature oxidation}

It is possible to distinguish two types of mechanism, those of computer aided generation and those which have been manually developed.

\section{2.a/ Mechanisms written without computer help}

A significant milestone in the modelling of low temperature oxidation of large alkanes 
was the appearance in 1975 of the Shell model developed by Halstead et al. [62]. This generalized model was based on formal reactions including initiation, chain propagation, degenerate branching and termination steps, with kinetic parameters fitted empirically. Following this, progress was made in the development of a priori reduced mechanisms (to be distinguished from a posteriori reduced mechanisms obtained by reduction techniques from detailed mechanisms), including elementary steps, but involving globalized species (alkanes were represented by $\mathrm{RH}$, all alkyl radicals by "R•", all peroxy radicals by "ROO•"...) such as the models of Cox and Cole [48], Hu and Keck [63] and the unified one of Griffiths et al. [23]. This last model was particularly well detailed for a reduced model, as it distinguished the different types of alkyl radicals and of isomerizations of peroxy radicals. The model of $\mathrm{Hu}$ and Keck [63] has been recently extended by Tanaka et al. [64] to produce a model that included 32 species and 55 reactions with a good prediction of the major autoignition features of n-heptane, iso-octane and mixtures. The development of the older reduced models has greatly helped the writing of the below-described detailed kinetic models.

Since their pioneering detailed modelling of the oxidation of n-butane at low and intermediate temperatures in 1988 [65], the team of Westbrook and Pitz in Livermore has performed an impressive work concerning the modelling of the oxidation and the autoignition of alkanes at low temperature. They have studied isomers of pentane [66-68] and hexane [69], but the largest progress was made for n-heptane [70], iso-octane [70] and the isomers of heptane [72], the mechanisms of which were developed in a systematical way using well-defined classes of reactions. This approach is very close to that of computer aided generation. These mechanisms were built in a stepwise fashion starting with small hydrocarbons and progressing to larger ones. That explains the very large size of these mechanisms: that for n-heptane includes 2450 reactions among 550 chemical and that of iso-octane involves 3600 reactions among 860 
species. The Livermore group has also been the first to propose a detailed modelling of the low temperature oxidation of two methyl esters, methyl formate and methyl butanoate [73]. Some qualitative agreement was observed when simulating experimental data obtained in closed vessels at subatmospheric pressure [74-75], but computations consistently indicated an overall reactivity higher by a factor of 10 to 50 compared to experiments.

Several mechanisms that cannot be analyzed in terms of classes of reactions have also be proposed. A mechanism for n-butane has been written by Kojima [76] and one for n-pentane by Ribaucour et al. [77]. Concerning ethers, modelling of the oxidation of TAME, ETBE and MTBE has been published by Böhm et al. [78-79]. In the case of esters, a mechanism of the oxidation of methyl butanoate has been proposed by Gaïl et al. [80] with validation over a wide range of operating conditions, from temperatures of $500 \mathrm{~K}$. In light of the scarcity of models concerning this class of compounds, let us also quote the mechanisms developed by Metcalfe et al. [81] for the high temperature oxidation of methyl butanoate and ethyl propanoate with validation using their shock tube data obtained at temperatures between 1100 and $1670 \mathrm{~K}$.

\section{2.b/ Computer aided mechanism generation}

The large number of reactions involved in low temperature oxidation mechanisms, with primary reactants of increased size, has led several teams to develop an approach based on an automatic generation of reactions. More details on automatic generation of mechanisms can be found in the well documented review of Tomlin et al. [82]. All the systems developed for low temperature oxidation mechanisms use a logical programming based on the definition of classes of elementary steps, as first proposed by Chinnick (1987) for pyrolysis [83].

In the early 90s, Chevalier et al. [84-86] have created a program for the automatic generation of mechanisms for the description of the low temperature oxidation of higher 
hydrocarbons. Their program was written in LISP and used a $\mathrm{C}_{0}-\mathrm{C}_{4}$ reaction base; but unfortunately their publications do not reveal many technical details. Mechanisms for the oxidation of n-heptane, n-decane and n-hexadecane were generated in order to model ignition delay times.

In order to find a trend between the production rate of branching agents and the blending octane number, two software for the generation of mechanisms for the low temperature oxidation of linear and branched alkanes were developed by Morley [87] and Blurock [88], respectively. The REACTION software developed by Blurock has recently been improved by Moréac et al. [89] and used to generate a low temperature oxidation detailed model for n-heptane and ndecane. The mechanism generation is based on the classes of reactions defined by Curran et al. [70]. This mechanism involves 506 species and 3684 reactions.

The first well validated oxidation mechanisms obtained with a computer aided method have been published by the team of Ranzi and Faravelli in Milano since 1995. In line with their previous work on pyrolysis [90-91], this team has produced semi-detailed mechanisms of the oxidation of alkanes [92-93]. An automatic generator of reactions (MAMOX) provides primary mechanisms (involving only the reactions of initial molecular reactants and their derived radicals) which are then lumped by grouping together the alkyl, peroxy, hydroperoxyalkyl and peroxy hydroperoxyalkyl radicals having the same carbon number and by considering that decomposition reactions can be globalized into single equivalent reactions whose stoichiometries are only weak functions of process temperature [94]. Secondary mechanisms (involving the reactions of molecular products formed in the primary mechanism) are also based on lumped reactions involving fractional stoichiometric coefficients and an extensively validated $\mathrm{C}_{0}-\mathrm{C}_{4}$ data base which also contains reactions of simple aromatic compounds such as benzene, toluene or methylnaphthalene [95]. Mechanisms are developed iteratively, i.e. a mechanism for n-heptane is 
based on previous mechanisms for n-pentane and n-hexane. Four well documented studies were presented, each including a detailed primary mechanism for n-pentane [94], n-heptane [96], iso-octane [97] and n-dodecane [92], respectively. Due to the large size of the $\mathrm{C}_{0}-\mathrm{C}_{4}$ data base, the mechanism for n-heptane involves more than 100 species and 2000 reactions and that of iso-octane 145 species and about 2500 reactions. An extension of this system has been made to model the oxidation of ethers (MTBE, ETBE, DIPE, TAME) $[92,98]$; but while they have been used successfully to model data obtained from $750 \mathrm{~K}$, these mechanisms include only hightemperature reactions.

Following an idea of Côme applied to thermal decompositions in the 80s [99-100], a team in Nancy has also been developing a system of automatic generation of reactions for the gasphase oxidation of hydrocarbons for several years [101]. This work has benefited from the development of THERGAS software initiated by Scacchi also in the mid 80s for the calculation of thermochemical data [28]. The software for the automatic generation of kinetic models (EXGAS), which has been based on the initial algorithm of Haux et al. [100], is now completely automatic for linear and branched alkanes (EXGAS-ALKANES) [24]. It provides reaction mechanisms made of three parts, as shown in figure 3:

- A comprehensive primary mechanism based only on elementary steps.

- $\mathrm{A} \mathrm{C}_{0}-\mathrm{C}_{2}$ reaction base, including all the reactions involving radicals or molecules containing less than three carbon atoms [102].

- $\quad$ A lumped secondary mechanism [103], which in order have a manageable size, involves lumped reactants (the molecules formed in the primary mechanism, with the same molecular formula and the same functional groups, are lumped into one unique species without distinguishing between the different isomers) and includes global reactions which produce, in the smallest number of steps, molecules or radicals whose reactions are included in the reaction 
bases.

During the generation of the mechanism, the thermochemical data of each species and the rate constant for each reaction are produced automatically by using THERGAS [28] and KINGAS [103] software, respectively. The kinetic data are either calculated for the elementary steps by means of thermochemical kinetics or estimated by means of correlations.

\section{FIGURE 3}

It is worth noting that contrary to the work performed in Livermore and in Milano, these mechanisms were not developed iteratively. Therefore the development of a mechanism for n-decane does not require the generation of the n-nonane mechanism as a prerequisite and so the size of these mechanisms is relatively restricted as shown in Table 2. Another important point about EXGAS is that the system of generation gives a large degree of choice for tailoring the sought mechanisms according to the conditions of study. All the generic reactions in the primary and the secondary mechanisms are not systematically activated; they can be chosen in a menu [104]. This system has been used to generate mechanisms for the low temperature oxidation of n-butane [24, 103], the isomers of pentane [24], 2-methyl-pentane [24], the isomers of heptane[24, 104], iso-octane $[24,104]$ and n-decane $[24,106]$. An extension of this system has also been made to model the oxidation of ethers (MTBE, ETBE) using comprehensive low temperature mechanisms [107].

\section{3/ Experimental results available for validation}

The experimental results concerning the autoignition and the oxidation below $900 \mathrm{~K}$ of alkanes from $\mathrm{C}_{4}$, which have been published since 1993, are shown in Tables 3, 4 and 5.

\section{TABLES 3 - 5}

For autoignition, two types of experimental facilities have been mainly used: high pressure shock tubes and rapid compression machines. In a shock tube, ignition delay time is 
usually measured behind a reflected shock wave, while the corresponding temperature is calculated from the incident shock wave velocity with an estimated error of around $20 \mathrm{~K}$. Four high pressure shock tubes have been used to work with air-hydrocarbon mixtures for pressures behind a reflected shock wave of up to 60 bar and then to observe autoignition at temperatures from $660 \mathrm{~K}$. The first results were obtained by the team of Adomeit [118-119,127] in Aachen for n-heptane, iso-octane and n-decane. The more recent studies of Davidson et al. $[121,126]$ in Standford, Herzler et al. [120] in Duisburg and Zhukov et al. [128] in Moscow were mostly in good agreement with these older results [118,127] and supported the observation of a pronounced low temperature NTC region. The study of Herzler et al. [120] has extended the range of studied equivalence ratios towards small values (up to 0.1), which are of interest for the development of HCCI engines. Zhukov et al. have also studied n-pentane [113] and n-hexane [114] for pressures up to 530 bar; for n-hexane, the experimental results were in good agreement with simulations using the model of Curran et al. [70].

Between 1993 and 2007 (the time period of interest in this review), six rapid compression machine facilities have been used to measure autoignition delay times for alkanes and, in some cases, to analyze pre-ignition products; they are:

1. at the University of Leeds with a compression time of $22 \mathrm{~ms}$ [111-112],

2. at the University of Lille with a compression time of $60 \mathrm{~ms}[108,110,116,122]$,

3. at the University of Galway with a compression time of $17 \mathrm{~ms}$ [115],

4. at M.I.T in Cambridge (USA) with a compression time of 19-30 ms [117],

5. at the University of Michigan with a compression time of about $100 \mathrm{~ms}$ [123-125,142]

6. at Case Western Reserve University in Cleveland with a compression time of 20-40 ms $[129,143]$.

The apparatuses of M.I.T and Case Western Reserve University have been designed to be used at 
pressures above 40 bar. In the six rapid compression machines, ignition delay time is measured starting from the end of the compression. The major problem in such an apparatus is the knowledge of the temperature $\left(\mathrm{T}_{\mathrm{c}}\right)$ of the compressed gas. It is usually calculated for an adiabatic core gas from the initial pressure $\left(\mathrm{P}_{0}\right)$ and temperature $\left(\mathrm{T}_{0}\right)$, the compressed pressure $\left(\mathrm{P}_{\mathrm{c}}\right)$ which can be experimentally measured and the ratio of specific heats $\left(\gamma=C_{p} / C_{v}\right)$ by the following equation [116],

$$
\int_{\mathrm{T} 0}^{\mathrm{Tc}} \frac{\gamma}{\gamma-1} \frac{\mathrm{dT}}{\mathrm{T}}=\ln \frac{\mathrm{P}_{\mathrm{c}}}{\mathrm{P}_{0}}
$$

While a simplified method has been proposed by Tanaka et al. [64] to consider wall heat transfer as the result of a volume expansion, several experimental studies have demonstrated that the details of the heat transfer in a rapid compression machine are quite complex, both with respect to the geometry and over the time history of the experiments [72,144-145]. This is of particular importance for the compounds with the longest ignition delay times and it has been shown that simulations with a simple physical model encounter problems in reproducing the experimental results obtained in Leeds for highly branched alkanes, which show a region of temperature around $750 \mathrm{~K}$, in which no autoignition was experimentally observed [112,146]. The experimental results obtained in Galway for highly branched alkanes show also a region of temperature in which no autoignition may be observed, or with a strongly pronounced NTC [115]. In addition, temperatures gradients have been measured in the combustion chamber of such an apparatus [146], which can greatly influence the potential measurement of products. With its longest compression time involving less turbulence formation, the machine of Lille has by far produced the largest number of results which have been successfully used to validate kinetic models for many components of gasoline, as well as for mixtures. Simulations display a satisfactory agreement when using results from this apparatus, albeit assuming adiabaticity even after long ignition delay times. 
Most studies in a shock tube or a rapid compression machine have been focused on species included in gasoline, isomers of pentane and heptane and iso-octane; however three independent studies have been devoted to n-decane [127-129] with good agreement in the measured delay times. It is worth noting for n-decane, the satisfactory agreement obtained between results measured in a rapid compression machine [129] and in a shock tube [127]. A similar agreement between data obtained in these two different types of experimental facilities was also observed for iso-octane $[119,123,126]$.

A last type of gas-phase apparatus, a spherical, stainless-steel vessel, has been used by Chandraratna and Griffiths (1994) [109] to investigate the conditions at which autoignition occurs in lean premixed n-butane + air mixtures. As reviewed by Pollard [47] and Griffiths [23], closed vessels have been much used before 1993 to study the low temperature oxidation of hydrocarbons and other gaseous organic compounds. Measurements of autoignition delay times in such an apparatus can be strongly sensitive to wall reactions, as shown for n-butane by Cherneskey and Bardwell in coated reactors [147].

Let us quote also experiments concerning the autoignition and the burning rate of fuel droplets in a heated gas, in some cases under microgravity. The obtained results can be a further test of kinetic mechanisms, even if the complexity of the physical model is strongly increased. A review on this topic has been published by Aggarwal in 1998 [148] and more recent results have been obtained for n-decane at a gas temperature below $900 \mathrm{~K}$ [149-150].

Autoignition data are of interest for the validation of kinetic models, because they are closely related to the phenomena occurring in engines. Nevertheless, as noted by Mittal et al. [151], any evaluation of a kinetic scheme by reference to ignition delay times must be treated with some caution when the kinetic uncertainties are not taken into account, since equivalent results may be predicted using very different sets of important parameters. It is then important to 
consider for validation different sources of data, including results obtained in reactors for slow oxidation.

A more limited number of experiments concerning the slow oxidation at temperature below $900 \mathrm{~K}$ have been published; the teams of D'Anna in Napoli [133], Cernansky in Drexel University [68,138-141] and Dryer in Princeton [136] have used adiabatic pressurized flow reactors and the teams of Dagaut in Orléans [131-132,137] (based on a reactor design first proposed by Matras and Villermaux [152]) and of D'Anna in Napoli [133-135] have developed pressurized isotherm jet-stirred reactors. The main advantage of the data obtained in reactors is to give information about not only the global reactivity of the system, as conversions of reactant and ignition delay times do, but also on the selectivities of the obtained products by using adapted analytical techniques, such as gas chromatography with mass spectrometry, flame ionization or thermal conductivity detection. For n-heptane, with a large dilution in inert gas, Dagaut et al. [131] have detected about fifty species, including large alkenes (heptenes) and cyclic ethers (substituted tetrahydrofurans) in a system which can be reproduced by the simplest physical model. It is worth noting that the study of Lenhert et al. [140-141] and by D'Anna [133] in flow reactors were concerned by the oxidation of n-dodecane and the autoignition of n-tetradecane, respectively, with these large compounds being representative of those included in diesel fuel.

The mechanisms described above have been validated using a large part of these experimental results. As it is of little interest to compare model performance in terms of the best reproduction of experimental data, only one example of the agreement obtained is presented in figures 4-6 in the case of n-heptane. These figures show that the models of Livermore [70], Milano [96] and Nancy [24] for the oxidation of n-heptane perform very similarly when reproducing data obtained in a shock tube [118], a jet-stirred reactor [132] and a rapid 
compression machine [116] (validation not found for the model of Milano). The three models reproduce very well the position of the NTC area and its variation with pressure which shifts towards higher temperature when pressure increases. This shift is due to the influence of pressure on the equilibrium of the addition reactions of molecular oxygen to the alkyl and hydroperoxyalkyl radicals [70]. It is interesting to note that ignition delay times obtained in a shock tube and in a rapid compression machine at the same pressure (average 3.2 bar) correspond to the same simulated curve as shown in figure 4 with the model of Nancy. However, the simulations made for the lowest pressure range (from 3 to 4 bar) correspond to the least satisfactory agreement obtained by the model of Nancy and are not presented by other authors. In a rapid compression machine, cool flame delay times (first ignition time) have also been experimentally measured and are well simulated by the models of Livermore and Nancy (see figure 6).

\section{FIGURES 4-6}

Validation is slightly less satisfactory for iso-octane [24,71,97], showing that some problems still remain for modelling highly branched alkanes. Well validated detailed modelling studies of the oxidation of n-decane and n-dodecane are still scarce [24,93]. The paper of Herzler et al. [120] concerning the autoignition of n-heptane in lean mixtures ( $\phi$ from 0.1 to 0.4 ) in a shock tube, shows that the mechanism of Curran et al. [70] reproduces the general trends in the temperature and equivalence ratio dependence of the ignition delay times well, but that the absolute values disagree by a factor of 2 . The same modelling results were obtained in Nancy [153], which can lead one to think that an important reaction in lean mixtures is not well understood. More experimental results in lean mixtures would therefore be valuable.

Simulations of the conditions under which autoignition occurs in lean premixed n-butane/air mixtures in a closed vessel were performed by the team of Griffiths using the 
models of Nancy and Livermore and compared to experimental data [109]. While the qualitative features are well accounted for, both models overpredict the autoignition temperatures at low concentrations of fuel [154].

The model of Milano has been successfully used to reproduce results of autoignition [149] and burning rates [150] of fuels droplets of $n$-decane in a heated gas at constant pressure [155]. The variation of the cool flame and the total ignition delay times with the ambient temperature was properly predicted.

Tables 6 presents the experimental results concerning the autoignition and the oxidation below $900 \mathrm{~K}$ of ethers and esters from $\mathrm{C}_{4}$, which have been published since 1993 and shows that these results are much less numerous than those for alkanes. The most investigated ether is MTBE, which is the only one for which autoignition delay times in a shock tube have been measured [119], but not yet modelled. No data have been obtained in a rapid compression machine. Several studies in pressurized isotherm jet-stirred reactors have been published $[98,107,156]$; the models of Milano and Nancy can correctly reproduce the measurements made by the team of Dagaut above $850 \mathrm{~K}$, but at lower temperature a catalytic reaction needs to be taken into account to explain the early formation of alcohol. Data have also been obtained in a pyrex closed vessel [54]. In this type of reactor, the model of Nancy fails to simulate the results for ETBE, which were found to be sensitive to wall effects, although it can satisfactorily predict the older data obtained by Brocard et al. for MTBE [53].

\section{TABLE 6}

To our knowledge, no experimental measurements concerning the oxidation of ethyl esters or the ignition of esters in a shock tube or a rapid compression machine have been published since 1993. Despite the environmental interest of this topic, the amount of experimental data concerning methyl esters is still very limited. Methyl butanoate is the most 
studied compound of this family, with measurements using flow and jet-stirred reactors performed by the teams of Dryer [80] and Dagaut [80,157], respectively, and leading to a reasonable agreement with the modelling of Gail et al. [80]. Flow reactor experiments were performed between 500 and $900 \mathrm{~K}$, but no NTC behaviour was observed. Jet-stirred experiments were also carried out in order to study the influence of an unsaturation in $\mathrm{C}_{4}$ methyl esters [157]: both fuels have similar reactivity, but methyl crotonate combustion produces much higher levels of soot precursors (acetylene, propyne, 1,3-butadiene). Although slightly outside the scope of this paper, recent measurements in coflowing laminar non-premixed flames for methyl butanoate, methyl isobutyrate and ethyl propionate [158] and in opposed-flow diffusion flames for saturated and unsaturated $\mathrm{C}_{4}$ methyl esters $[80,157]$ can also be quoted.

2.4/ Comparison between the major models in terms of elementary steps and associated rate constants

Whether they were generated by a computer aided system or by careful human analysis, the mechanisms of Livermore, Milano (the detailed primary mechanisms were analysed) and Nancy have all been developed in a systematic way by applying well defined classes of reactions. Table 7 presents a comparison of the different classes of reactions considered by the three teams.

\section{TABLE 7}

While the classes of reactions involved in the high temperature primary mechanisms (initiations, H-abstractions from alkane molecules, isomerizations and decompositions by $\beta$-scission of alkyl radicals) are the same whatever the team and will not be reviewed here, there are more differences concerning the low temperature primary and the secondary mechanisms. As the secondary reactions of alkenes are mainly important at high temperature, this paper will only focus on the reactions involved in the low temperature primary mechanism, on the secondary 
reactions of hydroperoxides and cyclic ethers and on the specificities of the reactions of ethers and esters.

In the models of Livermore, the rate coefficients of the reverse of each reaction were obtained by fitting of the rate constants computed from the thermochemistry. In Milano and Nancy, reverse reactions were considered only for additions to oxygen molecules and isomerizations, with specified rate constants in the team of Ranzi and Faravelli and with rate parameters automatically deduced by the solver from those of the direct reaction using thermodynamic data in EXGAS models. This latter method ensured the best consistency between kinetics and thermochemistry.

\section{4.a/ Reactions of alkyl radicals $(\mathrm{R} \bullet)$}

At low temperature, the main reactions of alkyl radicals take place with oxygen molecules. The current understanding of these reactions for small alkyl radicals, such as ethyl or propyl radicals, is in agreement with the fact that the $\mathrm{R} \bullet+\mathrm{O}_{2}$ reaction proceeds through a barrierless addition pathway to form the $\mathrm{RO}_{2} \bullet$ adduct, which will either be stabilized or react via a concerted elimination of $\cdot \mathrm{HO}_{2}$ to give the conjugated alkene [159]. The potential energy surfaces, calculated quantum mechanically by DeSain et al. [159], for the reactions of n-propyl radicals with oxygen molecules are displayed in figure 7 . An accurate representation of this type of reaction would require parameterizing the rate constants as a function of temperature and pressure, which has not yet been done for alkyl radicals containing a large number of carbon atoms. This explain why deviations were observed when modelling experimental data obtained at low pressures, as already mentioned for n-heptane.

\section{FIGURE 7}

Benson [29] proposed an average value of the rate constant of the addition of alkyl radicals to oxygen molecules of $2 \times 10^{12} \mathrm{~cm}^{3} \mathrm{~mol}^{-1} \mathrm{~s}^{-1}$ at $650 \mathrm{~K}$ and several studies have shown that 
this reaction displays a negative temperature dependence which is not is not the result of an energy barrier [160-161]. Lower values of the rate constant were measured for branched radicals (iso-butyl, neo-pentyl) than for straight-chain ones [160]. The rate constant of the reverse reaction is also of importance and can be deduced from microscopic reversibility.

At Livermore, the rate constant of the addition of alkyl radicals to oxygen molecules was assumed to be dependent on the type of alkyl radicals: $\mathrm{k}=4.52 \times 10^{12} \mathrm{~cm}^{3} \mathrm{~mol}^{-1} \mathrm{~s}^{-1}$ for primary radicals, $\mathrm{k}=7.54 \times 10^{12} \mathrm{~cm}^{3} \mathrm{~mol}^{-1} \mathrm{~s}^{-1}$ for secondary radicals and $\mathrm{k}=1.42 \times 10^{13} \mathrm{~cm}^{3} \mathrm{~mol}^{-1} \mathrm{~s}^{-1}$ for tertiary radicals [70]. In the detailed primary mechanisms of Milano, the values were taken to be equal to $9 \times 10^{11} \mathrm{~cm}^{3} \mathrm{~mol}^{-1} \mathrm{~s}^{-1}$ for all heptyl radicals [96] and $1 \times 10^{12} \mathrm{~cm}^{3} \mathrm{~mol}^{-1} \mathrm{~s}^{-1}$ for other alkyl radicals $[92,94,97]$. At Nancy, a unified approach has been proposed whatever the size of alkyl radicals [24], but taking into account the branching level through an additivity method :

$$
\mathrm{k}_{\mathrm{add}}=\mathrm{n}_{\mathrm{p}} \cdot \mathrm{k}_{\mathrm{p}}+\mathrm{n}_{\mathrm{s}} \cdot \mathrm{k}_{\mathrm{s}}+\mathrm{n}_{\mathrm{t}} \cdot \mathrm{k}_{\mathrm{t}}+\mathrm{n}_{\mathrm{q}} \cdot \mathrm{k}_{\mathrm{q}}
$$

with :

$\mathrm{n}_{\mathrm{p}}$ : number of primary groups $\left(\mathrm{CH}_{3}\right)$ linked to the radicalar atom of carbon,

$\mathrm{n}_{\mathrm{s}}$ : number of secondary groups $\left(\mathrm{CH}_{2}\right)$ linked to the radicalar atom of carbon,

$\mathrm{n}_{\mathrm{t}}$ : number of tertiary groups $(\mathrm{CH})$ linked to the radicalar atom of carbon,

$\mathrm{n}_{\mathrm{q}}$ : number of quaternary groups $(\mathrm{C})$ linked to the radicalar atom of carbon,

$$
\begin{array}{ll}
\mathrm{k}_{\mathrm{p}}=8.0 \times 10^{18} \mathrm{~T}^{-2.5} \mathrm{~cm}^{3} \cdot \mathrm{mol}^{-1} \cdot \mathrm{s}^{-1}, & \mathrm{k}_{\mathrm{s}}=9.0 \times 10^{18} \mathrm{~T}^{-2.5} \mathrm{~cm}^{3} \cdot \mathrm{mol}^{-1} \cdot \mathrm{s}^{-1}, \\
\mathrm{k}_{\mathrm{t}}=1.5 \times 10^{18} \mathrm{~T}^{-2.5} \mathrm{~cm}^{3} \cdot \mathrm{mol}^{-1} \cdot \mathrm{s}^{-1}, & \mathrm{k}_{\mathrm{q}}=1.0 \times 10^{18} \mathrm{~T}^{-2.5} \mathrm{~cm}^{3} \cdot \mathrm{mol}^{-1} \cdot \mathrm{s}^{-1} .
\end{array}
$$

This leads to values of about $2 \times 10^{12} \mathrm{~cm}^{3} \mathrm{~mol}^{-1} \mathrm{~s}^{-1}$ for heptyl radicals (in agreement with the values proposed by Benson [29]) and between $1.1 \times 10^{11} \mathrm{~cm}^{3} \mathrm{~mol}^{-1} \mathrm{~s}^{-1}$ and $2.8 \times 10^{12} \mathrm{~cm}^{3} \mathrm{~mol}^{-1} \mathrm{~s}^{-1}$ for octyl radicals at $600 \mathrm{~K}$. For these parameters, which have by far the highest sensitivity coefficient in the analyses of Buda et al. [24], there is clearly an important discrepancy between the low values used in Milano and Nancy and the higher ones used in Livermore, and no new experimental 
study of this reaction has been published since the end of the 80 s.

As they consider that the contribution of the reaction leading to $\cdot \mathrm{HO}_{2}$ radicals and alkenes decreases significantly with increasing number of atoms of carbon in the alkyl radical, due to an increased possibility of stabilisation of the adduct by vibrations, Curran et al. [70] neglect this class of reaction for alkyl radicals containing more than 4 atoms of carbon. As discussed by Walker and Morley [49], the mechanism of this reaction has been the subject of considerable controversy and the values of these rate parameters are not well known for alkyl radicals heavier than ethyl radicals. Nevertheless, the values used in Milano and Nancy are not so different. Ranzi et al. [92,94,96- 97] considered A-factors ranging from $1.5 \times 10^{11} \mathrm{~cm}^{3} \mathrm{~mol}^{-1} \mathrm{~s}^{-1}$ to $2.5 \times 10^{11} \mathrm{~cm}^{3} \mathrm{~mol}^{-1} \mathrm{~s}^{-1}$ (per abstractable $\mathrm{H}$-atom) and an activation energy between 1.5 and 6.2 $\mathrm{kcal} / \mathrm{mol}$. Buda et al. [24] proposed A-factors depending on the type of abstracted $\mathrm{H}$-atoms and ranging from $2.7 \times 10^{11} \mathrm{~cm}^{3} \mathrm{~mol}^{-1} \mathrm{~s}^{-1}$ to $1 \times 10^{12} \mathrm{~cm}^{3} \mathrm{~mol}^{-1} \mathrm{~s}^{-1}$ (per abstractable H-atom, for alkyl radicals containing more than four atoms of carbon) and an activation energy equal to $5 \mathrm{kcal} / \mathrm{mol}$ [162].

\section{4.b/ Reactions of peroxyalkyl radicals (ROO•)}

There are two types of reactions of peroxyalkyl radicals, isomerizations by internal transfer of H-atom and disproportionations with other radicals.

\section{4.b. $\alpha /$ Isomerizations}

An example of isomerization by internal transfer of an $\mathrm{H}$-atom is presented in figure 8 . Isomerizations with a transition state ring including from 5 to 8 members were taken into account in Livermore, from 5 to 7 members in Milano and from 4 to 8 members in Nancy.

\section{FIGURE 8}

The first values for the rate parameters of a whole series of isomerizations of peroxy radicals were obtained by Baldwin et al. [163] in the team of Hull by studying the chemistry 
associated with neopentylperoxy radicals when neopentane is added to slowly reacting mixtures of $\mathrm{H}_{2}+\mathrm{O}_{2}$ between 673 and $773 \mathrm{~K}$. This indirect measurement was dependent on the equilibrium constant of the addition of alkyl radicals with oxygen, the thermochemistry of which was a subject of controversy [161] in the late 80 s. Hughes et al. [164] in the team of Pilling have later studied the isomerization of neopentylperoxy radicals between 660 and $750 \mathrm{~K}$ using the laser flash photolysis/laser induced fluorescence technique and rescaled the values of Baldwin et al. by using a more accurate value for the equilibrium constant of the addition of alkyl radicals with oxygen [161]. Quantum mechanical calculations by Chan et al. [165] give activation energies between 5 and $7 \mathrm{kcal} / \mathrm{mol}$ higher than those proposed by the team of Pilling [161] and have not been considered in any models.

In Livermore [70], A-factors were calculated using RADICALC [166], a computer code that implements transition state theory and calculates the change in entropy when the radical moves to the transition state. In Milano, A-factors only depended on the size of the transition state ring. In Nancy, A-factors were mainly based on the changes in the number of internal rotations as the reactant moves to the cyclic transition state and were estimated using [53]:

$$
A=e^{1} \frac{k_{B} T}{h} \times r p d \times \exp \left[\frac{\left(\Delta n_{i . r o t .}^{*}\right) \times 3,5}{R}\right] s^{-1}
$$

with :

$$
\begin{array}{lll}
\mathrm{h} \quad \text { Planck constant, } & \mathrm{k}_{\mathrm{B}} \quad \text { Boltzmann constant, } \\
\mathrm{rpd} \quad \text { reaction path degeneracy }=\text { number of } & \Delta n_{i . r o t .}^{\neq} \text {change in the number of internal } \\
\text { identical abstractable } \mathrm{H} \text { atoms, } & \text { rotations as reactant moves to the } \\
& \text { transition state. }
\end{array}
$$

As proposed by Benson [29], activation energies for isomerization were set equal to the sum of the activation energy for $\mathrm{H}$-abstraction from the substrate by analogous radicals and the 
strain energy of the cyclic transition state.

Table 8 compares the values of A-factors and activation energies used by the groups of Livermore [70] Milano [92,94] and Nancy [24], with the ones recommended by the team of Pilling [161]. The parameters used by the group of Nancy were directly derived from the values proposed by the team of Pilling, while those of Livermore and Milano have been adjusted in order to reproduce experimental results.

\section{TABLE 8}

\section{4.b. $\beta /$ Disproportionations}

Above $600 \mathrm{~K}$, the rate constants of isomerizations are high enough for reactions of peroxy radicals with other radicals to be of negligible importance, as shown by the sensitivity analyses of Curran et al. [70] and Buda et al. [24]. This class of reaction was therefore not considered by Ranzi et al. [94] and only the reactions with $\cdot \mathrm{HO}_{2}$ radicals to give $\mathrm{ROOH}$ and $\mathrm{O}_{2}$, were taken into account by Buda et al. [24]. Deriving from parameters proposed for $\mathrm{CH}_{3} \mathrm{OO}$ • radicals [167], the rate constants of the disproportionation of peroxyalkyl radicals with $\cdot \mathrm{HO}_{2}$ radicals were very close in Livermore and Nancy: $1 \times 10^{11} \mathrm{~cm}^{3} \mathrm{~mol}^{-1} \mathrm{~s}^{-1}$ in Livermore [70] and to $2 \times 10^{11} \exp (2600 / \mathrm{T}) \mathrm{cm}^{3} \mathrm{~mol}^{-1} \mathrm{~s}^{-1}$ in Nancy [24].

\section{4.c/ Reactions of hydroperoxyalkyl radicals $(\bullet Q O O H)$}

There are three types of reactions of hydroperoxyalkyl radicals, addition to oxygen molecules, decomposition to give cyclic ethers and decompositions to give acyclic species.

\section{4.c. $\alpha /$ Addition to oxygen molecules}

The three research groups have taken the rate expressions of this class of reaction as identical to those for the addition of alkyl radicals to oxygen molecules.

\section{4.c. $\beta /$ Decomposition to give cyclic ethers}

An example of formation of cyclic ether is given in figure 9, in the case of the formation 
of a tetrahydrofuran, i.e. a cyclic ether including 5 atoms. The formation of oxirans, oxetans and tetrahydropyrans, containing 3,4 or 6 atoms, respectively, is also possible. This process competes directly with the addition of hydroperoxyalkyl radicals to oxygen, which leads ultimately to the formation of hydroperoxides, and has an inhibiting effect on the global reactivity. The rate constants of these reactions are thus very sensitive parameters, but a direct measurement is only available for the formation of $a$ four membered ring during the oxidation of neo-pentane [163] $\left(\mathrm{k}=2.0 \times 10^{11} \exp (-7830 / \mathrm{T}) \mathrm{s}^{-1}\right)$ and the parameters used for other cycles rely only on estimations. The models of Livermore and Nancy include the formation of cyclic ethers rings containing from three to six atoms. In the model of Milano the formation of six membered rings is neglected. Table 9 compares the values of A-factors and activation energies used by the three groups $[24,71,92]$.

\section{FIGURE 9}

\section{TABLE 9}

In the models of Milano [96,97], apart from the recent study of n-dodecane [92], A-factors were kept constant at $2.0 \times 10^{11} \mathrm{~s}^{-1}$, whatever the size of the ring. In the Livermore models [70], the n-dodecane model of Milano and the Nancy mechanisms [24], the loss in entropy due to the loss in free rotors when forming the cycle was taken into account leading to a decrease in the A-factor when increasing the size of the formed ring. Nevertheless, in the models of Livermore and Milano, there was a consistent decrease of the A-factor by 8 and 6.3, respectively, for each increase of one atom in the ring, while the decrease in the models in Nancy varied from 6 to 25. In the three research groups, activation energies have mainly been fitted to reproduce the distribution of ethers experimentally measured. Nevertheless, these values were consistent with a decrease of the ring strain energy when increasing the size of the cycle. This is not the case for the values calculated by quantum mechanical methods by Chan et al. [168], 
which lead to an activation energy of $15 \mathrm{kcal} / \mathrm{mol}$ for the formation of oxiranes, $24 \mathrm{kcal} / \mathrm{mol}$ for that of oxetanes, $16 \mathrm{kcal} / \mathrm{mol}$ for that of furans and $18 \mathrm{kcal} / \mathrm{mol}$ for that of pyrans.

\section{4.c. $\gamma /$ Decomposition to give acyclic species}

Figure 10 illustrates the different possibilities of decomposition. A hydroperoxyalkyl radical can decompose to give an alkene and a $\bullet \mathrm{HO}_{2}$ radical by breaking of a $\mathrm{C}-\mathrm{O}$ bond; this reaction was considered by the three groups. The breaking of a $\mathrm{C}-\mathrm{C}$ bond can lead either to an alkene and a smaller hydroperoxyalkyl radical, as also considered by the three groups, or to an unsaturated hydroperoxide molecule and a smaller alkyl radical, as taken into account only in Nancy. In the models of Livermore and Milano, the smaller hydoperoxyalkyl radical obtained is immediately decomposed to give an aldehyde and an $\bullet \mathrm{OH}$ radical. The breaking of an $\mathrm{O}-\mathrm{O}$ bond is only possible when the atom of carbon bound to an atom of oxygen bears also the radical center, which is only the case in the models of Nancy, due to the possibility of an isomerization involving a transition state including a four-membered ring. This channel leads to the formation of an aldehyde or a ketone and an $\bullet \mathrm{OH}$ radical. Table 10 compares the values of A-factors and activation energies used by the three groups $[24,71,92]$.

\section{FIGURE 10}

\section{TABLE 10}

The formation of an alkene and an $\cdot \mathrm{HO}_{2}$ radical is an important channel in the models of Livermore, because it is the only way for the formation of alkenes including the same number of carbon atoms as the reactant. The rate parameters of this reaction in Livermore were deduced using thermochemistry from those of the reverse reaction of the addition of $\bullet \mathrm{HO}_{2}$ radicals to alkenes, as proposed by Chen and Bozzelli [169] for ethylene, propene and isobutene. The rate constant used in Nancy was taken from Baldwin et al. [170] and has been deduced also from that of the reverse reaction of the addition of $\cdot \mathrm{HO}_{2}$ radicals to small alkenes. 
Because of their importance in the high-temperature oxidation mechanism, the rate constant of the decomposition involving the breaking of a $\mathrm{C}-\mathrm{C}$ bond are relatively well known $[33,34,84,171]$ and a good agreement is observed between the values used in Livermore and Nancy. The value for the decomposition by breaking of an O-O bond used in Nancy was taken from Chevalier et al. [84].

\section{4.d/ Reactions of peroxyhydroperoxyalkyl radicals $(\cdot \mathrm{OOQOOH})$}

In the same way as peroxy radicals, peroxyhydroperoxyalkyl radicals can isomerizes by an internal transfer of an H-atom. In the system of Nancy, the dihydroperoxyalkyl radicals $\left(\cdot \mathrm{U}(\mathrm{OOH})_{2}\right)$ obtained can react by decomposition to give cyclic ethers, alkenes, ketones and aldehydes including a hydroperoxide function. But, during their study of the oxidation of n-heptane, Glaude et al. [104] have noticed that 51 hydroperoxyalkyl radical isomers $(\cdot \mathrm{QOOH})$ were produced leading to the formation of 51 peroxyhydroperoxyalkyl radical isomers $(\bullet \mathrm{OOQOOH})$ by addition of oxygen, 162 dihydroperoxyalkyl radical isomers $\left(\cdot \mathrm{U}(\mathrm{OOH})_{2}\right)$ by isomerizations of these $\cdot \mathrm{OOQOOH}$ radicals and numerous hydroperoxide species which decomposed rapidly by degenerate branching steps and led to smaller species. The nature of these hydroperoxide species had no kinetic importance, as their decomposition was governed by the breaking of the $\mathrm{O}-\mathrm{OH}$ bond. Therefore, in line with the work of Livermore and Milano, the models of Nancy can be simplified on request by the izomerisation and the decomposition being globalized into a single step. This step leads to the formation of a ketohydroperoxide $\left(\mathrm{C}_{\mathrm{n}-1} \mathrm{H}_{2 \mathrm{n}-1} \mathrm{OOHCO}\right)$ with a rate constant equal to that for isomerization. But, while in Livermore and Milano, only the isomerization abstracting an $\mathrm{H}$-atom bound to the atom of carbon bound to an oxygen atom (see figure 11) was considered, all the possible isomerizations were taken into account in Nancy. In Livermore, isomerizations of $\bullet \mathrm{OOQOOH}$ radicals deriving from the initial $\mathrm{H}$-abstraction of a tertiary $\mathrm{H}$-atom from the parent fuel and involving the transfer of alternative 
$\mathrm{H}$-atoms (i.e. not bound to the atom of carbon bound to an oxygen atom, as this reaction is not possible) have been considered. A promoting effect of this reaction on the computed ignition rates has been shown [72].

In the three research groups, the rate constants of these isomerizations were taken equal to those of peroxy radicals. In the case of an isomerization involving the transfer of an $\mathrm{H}$-atom bound to a carbon atom bound to an oxygen atom, as shown in figure 11, the activation energy has been reduced by $3 \mathrm{kcal} / \mathrm{mol}$ in Livermore [70] and by $2 \mathrm{kcal} / \mathrm{mol}$ in Nancy [24], in order to take into account the fact that this $\mathrm{H}$-atom can be more easily removed.

\section{FIGURE 11}

\section{4.e/ Secondary reactions of hydroperoxides}

Hydroperoxide species are degenerate branching agents, which can easily decompose by breaking an $\mathrm{O}-\mathrm{OH}$ bond and induce an increase of the amounts of $\bullet \mathrm{OH}$ radicals which ensure the propagation of the reaction. The reactivity of alkanes is directly related to the amount of hydroperoxides formed and the rate constant of the decomposition of these compounds is then an important parameter.

The values used by the three research groups have been derived from a measurement of Sahetchian et al. [172] for the decomposition of hydroperoxides and were the following:

- $\mathrm{k}=1.5 \times 10^{16} \exp (-21000 / \mathrm{T}) \mathrm{s}^{-1}$ for every hydroperoxides in Livermore [70],

- $\mathrm{k}=2 \times 10^{14} \exp (-20500 / \mathrm{T}) \mathrm{s}^{-1}$ for hydroperoxides deriving from $\mathrm{n}$-pentane [94], $\mathrm{k}=1 \times 10^{15} \exp (-20500 / \mathrm{T}) \mathrm{s}^{-1}$ for hydroperoxides deriving from $\mathrm{n}$-heptane [96] and n-dodecane [92] and $\mathrm{k}=1 \times 10^{16} \exp (-20500 / \mathrm{T}) \mathrm{s}^{-1}$ for hydroperoxides deriving from iso-octane [97], in Milano,

- $\mathrm{k}=1.5 \times 10^{16} \exp (-21200 / \mathrm{T}) \mathrm{s}^{-1}$ for hydroperoxides deriving from initial linear reactant and $\mathrm{k}=0.3 \times 10^{16} \exp (-21200 / \mathrm{T}) \mathrm{s}^{-1}$ for hydroperoxides deriving from initial branched 
reactant, in Nancy [24].

As explained previously, in order to limit the size of the mechanism, the secondary mechanisms developed in Milano and Nancy did not contain elementary steps, but globalized reactions. The lower value of the rate constant of branched compounds proposed in Nancy reflects the fact that the decomposition of hydroperoxides deriving from branched alkanes leads to less reactive molecules and radicals than in the case of straight-chain alkanes.

\section{4.f/ Secondary reactions of cyclic ethers}

In the mechanisms of the three groups, the reactions of cyclic ethers proceed first by an $\mathrm{H}$-abstraction. In Livermore [70], $\mathrm{H}$-abstractions by $\cdot \mathrm{HO}_{2}$ and $\bullet \mathrm{OH}$ radicals have been considered; while in Nancy, $\bullet \mathrm{H}$ atoms, $\bullet \mathrm{HO}_{2}, \cdot \mathrm{OH}, \cdot \mathrm{CH}_{3}, \cdot \mathrm{CH}_{3} \mathrm{O}_{2}$ and $\bullet \mathrm{C}_{2} \mathrm{H}_{5}$ radicals did react. In Milano, the abstracting radicals were all the radicals for which the abstraction reaction competes with their $\beta$-scission decomposition.

The radicals obtained can decompose to give either smaller acyclic oxygenated molecules or radicals and alkenes or alkyl radicals. In Milano and Livermore, the H-abstractions and the decompositions were globalized into a single step. There was a specificity in Nancy for this secondary mechanism: the radicals obtained from cyclic ethers including more than 3 atoms can also react with oxygen as shown in figure 12 . The reactions with oxygen involved the classical sequence of oxygen addition, isomerization, second oxygen addition, second isomerization and beta-scission to led to the formation of hydroperoxydes, degenerate branching agents, which decomposed to give $\cdot \mathrm{OH}$ free radicals and several molecules or radicals whose reactions were included in the $\mathrm{C}_{0}-\mathrm{C}_{2}$ reactions base [162]. This additional degenerate branching step increased the reactivity of the system and is a source of $\mathrm{CO}_{2}$ at low temperature.

\section{FIGURE 12}

In Livermore, the rate constants for the $\mathrm{H}$-abstractions depended on the type of abstracted 
$\mathrm{H}$-atom (primary, secondary, tertiary) and on whether the atom of carbon bound to it is bound or not to an atom of oxygen. In Milano and Nancy, due to the lumping of the primary products, such a detailed treatment was not possible.

\section{4.g/ Classes of reactions and kinetic data specific to the oxidation of acyclic ethers}

The only class of reactions specific to ethers is a molecular reaction involving a four-membered transition state and leading to the formation of an alcohol and an alkene molecule as shown in figure 13a. The rate constants used in the models were in line with previous work of the literature $[57,173]$ and were the following :

- $\mathrm{k}=2.0 \times 10^{14} \exp (-29440 / \mathrm{T}) \mathrm{s}^{-1}$ for MTBE and TAME and $\mathrm{k}=2.0 \times 10^{14} \exp (-28940 / \mathrm{T}) \mathrm{s}^{-1}$ for ETBE and DIPE, in Milano [92],

- $\mathrm{k}=1.6 \times 10^{14} \exp (-29690 / \mathrm{T}) \mathrm{s}^{-1}$ for MTBE and $\mathrm{k}=1.6 \times 10^{14} \exp (-30170 / \mathrm{T}) \mathrm{s}^{-1}$ for ETBE, in Nancy [107].

\section{FIGURE 13}

While they are a class of reactions included in high temperature models, the decompositions of alkyl radicals by $\beta$-scission involving the breaking of the relatively weak C-O bond are of importance even at low temperature. These reactions have an activation energy from 1 to $3 \mathrm{kcal} / \mathrm{mol}$ lower than that involving the breaking of a C-C bond $[98,107]$. In Milano, these decompositions were globalized into a single step to give $\mathrm{C}_{1}-\mathrm{C}_{4}$ species [98]; in Nancy, they can lead to the formation of alkoxy radicals $(\mathrm{RO} \bullet)$ which can also easily decompose to give an alkyl radical and a ketone or an aldehyde, with a low activation energy, i.e. $15 \mathrm{kcal} / \mathrm{mol}$ [107].

\section{4.h/ Classes of reactions and kinetic data specific to the oxidation of methyl and ethyl esters}

The few models [74,80-81] available for the oxidation of esters have been based on the same classes of reactions as for alkanes. The only class of reactions specific to esters is 
molecular reactions. While this class of reaction has been found negligible for methyl esters [81], the unimolecular elimination involving a six-membered transition state and leading to the formation of ethylene and an acid molecule has been proven to be of importance for ethyl esters by Metcalfe et al. [81] and Schwartz et al. [158]. An example of this reaction is shown for methyl propanoate in figure 13b; the rate constant proposed by Metcalfe et al. [81] was: $\mathrm{k}=4.0 \times 10^{12} \exp (-25163 / \mathrm{T}) \mathrm{s}^{-1}$.

As in the case of ethers, the presence of oxygen atoms involves differences in the activation energies of some $\beta$-scission decompositions. This has been discussed by El-Nahas et al. [174]. Changes can also be expected for the rate constants of isomerisations of peroxy radicals and the formation of cyclic ethers.

\section{5/ Conclusion on the modelling of the oxidation of alkanes, ethers and methyl and ethyl esters}

Several models exist for the oxidation of alkanes representative of those contained in gasoline, notably n-heptane and iso-octane. However, the modelling of the oxidation of compounds representative of those present in diesel fuel has not been much developed. The models proposed for alkanes containing from 4 to 8 carbon atoms can correctly reproduce most of the results published in the literature, apart from those obtained in very lean mixtures or with highly branched compounds. There is a lack of experimental data for very rich mixtures (equivalence ratio above 3) in well validated experimental systems.

Considering the discrepancies of the models in terms of classes of reactions and rate constants, and the weakness of the basis on which these rate parameters have been estimated, the good results obtained by the existing models are almost surprising. Differences in the used thermochemical properties, which were not reviewed here, probably partly compensate for the difference in kinetic data. The only reliable estimations of rate parameters were deduced from a few excellent studies by the teams of Walker and Pilling performed before 1995. 
If an increased accuracy of the prediction of the models is needed, additional experimental and theoretical work on the elementary steps described above would be of great interest. This will be particularly true when not only a calculation of global reactivity, such as what is given by autoignition delay times, will be required, but also the prediction of minor pollutants which can have some impact on the chemistry of the earth's atmosphere: alkenes, dienes, aldelydes, acids. More studies are especially needed to define better the rate constants of the reactions of different types of alkyl radicals with oxygen molecules by addition or through the formation of alkenes, the isomerisation of peroxy radicals, the formation and the consumption of cyclic ethers and the decomposition of hydroperoxides. This should be achieved by calculations based on quantum mechanics [175-178] or master equation [179] methods and confirmed by some well designed experimental results.

The models and experimental results are far less abundant in the case of ethers and esters than in the case of alkanes. No model can reproduce autoignition delay times for these compounds and experimental data are available only for MTBE. More studies about ETBE, which can be considered as a semi-renewable compound, since the raw material used for its production - ethanol - derives from biomass [180], and about esters more representative of those actually present in biodiesel could certainly be of great interest. Since the experimental investigation and the development of detailed mechanisms for the low temperature oxidation of saturated and unsaturated esters containing up to 19 atoms of carbon will certainly be difficult, useful information should certainly be gained by using lighter compounds, e.g. $\mathrm{C}_{6}-\mathrm{C}_{10}$ methyl esters, as model molecules.

\section{3/ ALKENES}

The presence of a double bond in alkene molecules has led kineticists to make an 
additional step in the complexity of the chemistry of low temperature oxidation. The radicals directly deriving from the reactant are no longer of a single type, as alkyl radicals from alkanes, but of at least three types, as shown in Figure 14, alkylic and allylic alkenyl radicals being obtained by $\mathrm{H}$-abstraction and hydroxyalkyl radicals being obtained by addition of $\bullet \mathrm{OH}$ radicals to the double bond. This explains the scarcity of models related to alkenes, representative of the compounds present in gasoline, even if these unsaturated compounds are the major products of the oxidation of alkanes, with yields of $65 \%$ for butyl radicals and about $50 \%$ for larger alkyl radicals [49], and some of them have an important kinetic effect as they can lead to resonance stabilized radicals. Experiments are also made more difficult when using these unsaturated reactants as the possibilities of polymerisation are favoured.

\section{FIGURE 14}

\section{1/ Main chemical features of their oxidation}

The largest contribution to the understanding of the reactions of alkenes at low temperature has been made by the team of Hull. A study of the addition of alkenes from $\mathrm{C}_{2}$ to $\mathrm{C}_{5}$ to slowly reacting mixtures of $\mathrm{H}_{2}+\mathrm{O}_{2}$ at $750 \mathrm{~K}$ allowed Baldwin and Walker [181] to review the elementary reactions specific to the oxidation of alkenes in 1981. Further improvements of the proposed rate constants were progressively proposed [182-185] and reviewed in 1997 by Morley and Walker [49]. A large number of rate constants for the reactions of alkenes have also been proposed by Tsang for propene [35].

The typical reactions which are important in the low temperature of alkenes from $\mathrm{C}_{4}$ are illustrated in figure 15 by a rate of reaction analysis performed for the oxidation of 1-pentene at $800 \mathrm{~K}$, with a detailed kinetic model [186] which will be further described next.

\section{FIGURE 15}

The reactions occuring from the initial alkene molecules are of two types, H-abstractions 
by oxygen molecules or by $\bullet \mathrm{OH}$ radicals and additions of atoms or radicals across the double bond.

When $\mathrm{H}$-abstraction occurs at the atom of carbon in a $\beta$ position to the double bond, electron delocalisation occurs in the emerging alkenyl radical (allylic 1-penten-3-yl radical in figure 15) with several significant consequences. The enthalpy of reaction of the initiations with oxygen molecules leading to allylic radicals is about $15 \mathrm{kcal} / \mathrm{mol}$ lower than those leading to alkyl radicals, but the A-factors are lower by about a factor of 10 due to the reduction of the entropy of activation, caused by the loss of one rotation in the delocalized radical [185]. The stability of allylic radicals favours recombination reactions, with $\mathrm{HO}_{2}$ radicals especially, which is a source of hydroperoxides. As mentioned by Knyazev and Slagle [187], due to the electron delocalization, an allylic radical can lead to the formation of two peroxyalkenyl radicals. Lodhi and Walker [183] have proposed a mechanism by which peroxyallyl radicals react by an internal addition to the double bond and lead to the formation of formaldehyde and $\bullet \mathrm{CH} 2 \mathrm{CHO}$ radicals. The stability of allylic radicals explains why alkenes always have a larger octane number than the alkane of same structure; e.g. $\mathrm{RON}$ is 24.8 for $\mathrm{n}$-hexane $(\mathrm{MON}=26)$ and $76.4(\mathrm{MON}=$ 63.4) for 1-hexene, $92.7(\mathrm{MON}=80.8)$ for 2-hexene and $94(\mathrm{MON}=80.3)$ for 3-hexene (the octane number is the highest for the isomers which can lead to two different allylic radicals) [11]. While the difference between RON and MON (sensitivity) is weak for alkanes as shown in Table 2, it is far larger for alkenes. The sensitivity is related to the temperature dependence of the rate of the reactions leading to autoignition [188].

Below $1000 \mathrm{~K}, \mathrm{H}$-abstractions from atoms of carbon engaged in the double bond can be neglected. The H-abstractions, occurring at the atom of carbon in a $\gamma$ or further position to the double bond, involve the formation of alkylic alkenyl radicals (1-penten-4-yl and 1-penten-5-yl radicals in figure 15), which have a reactivity very close to that of alkyl radicals, with 
nevertheless, a possibility of the formation of dienes and of cyclization. It is worth noting that unsaturated ethers could be obtained if the mechanism proposed for alkyl radicals is taken into account, as shown in figure 15.

Additions across the double bond occur mainly with $\bullet \mathrm{H}$ atoms and $\bullet \mathrm{OH}$ and $\bullet \mathrm{HO}_{2}$ radicals. For asymmetric alkenes, two adducts are formed for each radical undergoing addition. Additions of $\bullet \mathrm{H}$ atoms lead to alkyl radicals (2-pentyl radicals in figure 15; 3-pentyl was not shown, because its rate of production was too small). Walker et al. [182] have shown that the addition of $\cdot \mathrm{HO}_{2}$ radicals does not lead to the formation of hydroperoxyalkyl radicals, but directly to the formation of oxiranes and $\bullet \mathrm{OH}$ radicals. Kinetic evidence for the formation of oxiranes and alkoxy radicals ( $\mathrm{RO} \bullet$ ) from the reactions of alkenes and $\mathrm{RO}_{2} \bullet$ radicals has been found by Stark [189]. The addition of $\bullet \mathrm{OH}$ radicals lead to hydroxyalkyl radicals (1-hydroxypenten-2-yl and 2-hydroxy-penten-1-yl radicals in figure 15), the reactivity of which is close to that of alkyl radicals and lead to a potential formation of hydroxy cyclic ethers. The isomerization of hydroxyalkylperoxy radicals can occur through the mechanism proposed by the team of Waddington [190] and leads to the formation of aldehydes and $\bullet \mathrm{OH}$ radicals, as shown in figure 16.

\section{FIGURE 16}

These different channels lead to the formation of hydroperoxide species, the decomposition of which promotes the reactivity in the same way as during the oxidation of alkanes. The reversibility of the addition of oxygen to alkylic alkenyl and hydroxyalkyl radicals, which react very much as alkyl radicals, is responsible for the existence of a negative temperature coefficient (NTC) regime.

\section{2/ Low temperature oxidation models and experimental results available for their validation}

The first detailed kinetic models for an alkene heavier than ethylene were proposed for 
propene. The team of Livermore was again leading the way in 1989, with a mechanism considering only one addition to oxygen [191], followed ten years later by the team of Nancy using a version of EXGAS extended to alkenes [192]. The purpose of these two mechanisms was to reproduce results obtained in a static reactor from 580 to $740 \mathrm{~K}$ by Wilk et al. [193]. The mechanism of Nancy showed that the observed NTC was mainly due to the reversibility of the addition to oxygen of the adduct, $\cdot \mathrm{C}_{3} \mathrm{H}_{6} \mathrm{OH}$ which, via a mechanism similar to that of alkyl radicals and involving two additions to oxygen, yielded degenerate branching agents. Table 11 summarizes the experimental data available for the model validation in the case of alkenes containing more than three carbon atoms.

\section{TABLE 11}

Concerning $\mathrm{C}_{4}$ alkenes, a low temperature mechanism for the oxidation of 1-butene, 2-butene and iso-butene has been proposed by Pitz et al. [194] to model combustion products in an engine. Two mechanisms have been proposed by Dagaut and Cathonnet [195] and by Baugé et al. [196] to model the oxidation of iso-butene in jet stirred reactors. But, while they aimed at modelling results below $900 \mathrm{~K}$, these two mechanisms considered only high temperature reactions.

The first modelling study concerning alkenes of $\mathrm{C}_{5}$ has been published by Ribaucour et al. [77]. A mechanism including 888 reactions and 179 species has been proposed to reproduce autoignition delay times measured in a rapid compression machine [197]. The model allowed the experimental values of autoignition delay times to be satisfactorily enough modelled, but the NTC zone was not really captured. The abstractions of alkylic $\mathrm{H}$-atoms, the isomerizations of the peroxy radicals deriving from the successive additions of hydroxyl radicals and oxygen molecules or the formations of unsaturated cyclic-ethers have not been considered in this model.

A modelling of the oxidation of 1-pentene has very recently been published by the team 
of Ranzi and Faravelli [201-202] with validation using data in a rapid compression machine [197], but the building of the mechanism was not much detailed.

In recent years, the team of Nancy has improved the new version of EXGAS extended to alkenes in order to model the oxidation of alkenes, representative of those present in gasoline. Touchard et al. [186] have proposed mechanisms for 1-pentene and 1-hexene, which include 3385 reactions involving 837 species and 4526 reactions involving 1250 species, respectively. These mechanisms were validated using data measured for the autoignition of 1-pentene [197] and 1-hexene [199] in a rapid compression machine, showing well the difference of reactivity between the two compounds (RON is 90.9 for 1-pentene and 76.4 for 1-hexene [11]) as displayed in figure 17, and for the oxidation of 1-pentene in a flow tube [198].

\section{FIGURE 17}

Vanhove et al. [199] have measured cool flame and autoignition delay times, as well as the formation of 25 pre-ignition products for the 3 linear isomers of hexene in a rapid compression machine. Data in a jet-stirred reactor have also been measured for the oxidation of 1-hexene by Yahyaoui et al. [200] at temperatures from $750 \mathrm{~K}$; simulations performed using the high temperature mechanism proposed by the authors strongly underestimate the formation of products below $850 \mathrm{~K}$. The data measured by Vanhove et al. [199] for 2-hexene and 3-hexene and by Tanaka et al. [117] for linear isomers of heptene have not yet been modelled. To our knowledge, no data for heavier alkenes are available.

As shown in figure 18 for 1-hexene, the models of Nancy reproduce within a factor 2.5 the formation of dienes, saturated cyclic ethers and $\mathrm{C}_{6}$ aldehydes, but deviate more for the formation of unsaturated cyclic ethers and smaller aldehydes, and predict the formation of hydroxy cyclic ethers, which has not yet been experimentally demonstrated. Concerning unsaturated cyclic ethers, Vanhove et al. [199] have analysed 2-methyl-4-vinyl-oxetane, 2-vinyl- 
tetrahydrofuran and 2-ethyl-2,5-dihydrofuran (the structures of which are given in figure 19) during the oxidation of 1-hexene. The formation of 2-methyl-2,5-dihydrofuran was observed by Prabhu et al. during the oxidation of 1-pentene [198]. These unsaturated cyclic ethers were amongt those proposed by the models of 1-pentene and 1-hexene of Nancy [203], but the predicted quantities were much overestimated.

\section{FIGURES 18-19}

\section{4/ Elementary steps and associated rate constants specific to the reactions of alkenes}

The purpose of the following section is to review the differences in elementary steps and rate constants existing in the mechanisms for the low temperature oxidation of alkenes and to make a comparison to what has been previously described for alkanes. The data will be taken from the work of Nancy [186,192,203-204], as it is the best documented source of comprehensive models for alkenes representative of those included in gasoline. The rate constants for the reactions important at high temperature, molecular reactions (ene and retro-ene reactions), unimolecular and bimolecular initiations, H-abstractions from alkenes by small radicals, isomerization of alkylic radicals, decompositions by $\beta$-scissions, can be found elsewhere [205-208].

An up-date of THERGAS software for the estimation of thermochemical data [28] was also necessary, as many groups related to unsaturated compounds were missing in the tables of Benson [29] and some bond dissociation energies had to be re-evaluated; this was made using mainly the work of Ritter and Bozzelli [25], Knyazev and Slagle [187], Tsang [209], Luo [210] and Denisov and Denisova [211].

\section{4.a/ Additions to the double bond}

Table 12 presents the rate constants used for the additions of small radicals to the double bond. These values were derived from data proposed for propene [35,91]. In the case of the 
additions of $\cdot \mathrm{HO}_{2}$ radicals, the direct formation of oxiranes and $\bullet \mathrm{OH}$ radicals has been considered, while in the case of the addition of $\bullet \mathrm{H}$ atoms and $\bullet \mathrm{OH}$ and $\cdot \mathrm{CH}_{3}$ radicals the formation of the two adducts has been taken into account. Several studies [192,195-196,212] have indicated that when temperature increases the adduct cannot be stabilized, but breaks down into decomposition products. A direct addition/decomposition leading to aldehydes (e.g. formaldehyde and butanal in the case of 1-pentene) and alkyl radicals (e.g. methyl and butyl radicals in the case of 1-pentene) has then been written (for temperatures above $800 \mathrm{~K}$ ), but the transition between the two channels was not well defined and should be better investigated.

\section{TABLE 12}

\section{4.b/ Reactions of alkenyl and hydroxyalkyl radicals}

As for alkyl radicals, the main low temperature reactions of alkenyl and hydroxyalkyl radicals take place with oxygen molecules. The rate parameters of these reactions are summarized in Table 13. These radicals can all react by addition with oxygen molecules to form peroxy radicals, or by oxidation to gives dienes, hydroxyalkenes, aldehydes or ketones and $\bullet \mathrm{HO}_{2}$ radicals. These elementary reactions have not been much studied, and the proposed rate parameters had to be mostly estimated. As shown in figure 15 for 1-penten-3-yl radicals, the addition to oxygen of the allylic alkenyl radicals can lead to two different peroxy radicals; a branching ratio of 0.5 being considered for both channels.

\section{TABLE 13}

Allylic radicals can also react by termination steps, mainly by combination with $\cdot \mathrm{HO}_{2}$ radicals to give an hydroperoxide $\left(\mathrm{k}=1.0 \times 10^{15} \mathrm{~T}^{-0.8} \mathrm{~mol}^{-1} \mathrm{~cm}^{3} \mathrm{~s}^{-1}\right.$ [192]), the decomposition of which is a source of unsaturated aldehydes, such as acrolein. In the case of long chain alkenes, the possible cyclization of alkylic alkenyl radicals was taken into account, with an A-factor of $1.4 \times 10^{11} \mathrm{~s}^{-1}$ and an activation energy of $\mathrm{E}=16.2 \mathrm{kcal} / \mathrm{mol}$ as proposed by Gierczak et al. [216]. 


\section{4.c/ Reactions of peroxyradicals}

The same two types of reactions as for peroxyalkyl radicals, isomerizations by internal transfer of $\mathrm{H}$-atom and disproportionations with other radicals, have been considered. The rate constants of disproportionations have been taken to be equal to those of peroxyalkyl radicals previously presented (paragraph 2.4.b. $\beta$ ).

In the case of isomerizations, the rate parameters were obtained by the same method as presented previously (paragraph 2.4.b. $\alpha$ ) with slight adjustments:

- The activation energy of the abstraction of a hydrogen atom from an atom of carbon linked to an atom of oxygen in hydroxyperoxyalkyl radicals was considered to be $2 \mathrm{kcal} / \mathrm{mol}$ lower than from an atom of carbon linked to only atoms of carbon or of hydrogen, as in the case of hydroperoxyperoxyalkyl radicals.

- For the isomerization of hydroxyperoxyalkyl radicals involving the transfer of an H-atom from the $\mathrm{OH}$ group, the cyclic transition state decomposed and gave two aldehydes and $\bullet \mathrm{OH}$ as proposed by Stark and Waddington [190] with an energy for the abstraction of the H-atom of $18.6 \mathrm{kcal} / \mathrm{mol}$, as proposed by Ranzi et al. [95] for the reaction of $\mathrm{CH}_{3} \mathrm{O}_{2} \bullet$ radicals with methanol.

- The activation energy of the abstraction of a hydrogen atom leading to an allylic radical was lowered by $2-3 \mathrm{kcal} / \mathrm{mol}$ compared to that forming an alkyl radical.

- The strain energy of the cyclic transition states containing two oxygen atoms and a double bond was taken as equal to $15 \mathrm{kcal} / \mathrm{mol}$ (for a five membered ring), $10 \mathrm{kcal} / \mathrm{mol}$ (for a six membered ring), $1 \mathrm{kcal} / \mathrm{mol}$ (for a seven membered ring) and $0 \mathrm{kcal} / \mathrm{mol}$ (for an eight membered ring) according to the differences of ring correction between saturated and unsaturated cycles proposed by Benson [29].

A third kind of reaction has been considered for allylic radicals, the internal 
addition/decomposition proposed by Lodhi and Walker [183] with, $\mathrm{k}=1.7 \times 10^{9} \mathrm{~T} \exp (13200 / \mathrm{T})$ $\mathrm{mol}^{-1} \mathrm{~cm}^{3} \mathrm{~s}^{-1}$ ). Figure 20 illustrates this reaction for 1-penten-3yl-peroxy radicals. As for isomerizations, the A-factor was estimated from the changes in the number of internal rotations ( 2 rotations are lost) when the reactant moves to give the transition state and the activation energy from the sum of the energy for the addition and the strain energy of the four membered cyclic transition state.

\section{FIGURE 20}

A more accurate estimation of the parameters related to the reaction of peroxy radicals deriving from alkenes using theoretical calculations and a better consideration of steric effects, would certainly be useful to improve the modelling of the formation of products during the oxidation of large alkenes.

\section{4.d/ Reactions of hydroperoxyradicals}

The same three types of reactions have been considered as for hydroperoxyalkyl radicals deriving from alkanes, addition to oxygen molecules, decomposition to give cyclic ethers and decompositions to give acyclic species, using the same rate constants with slight changes:

- Cyclic ethers including rings, which contain from 3 to 6 members, a double bond or an alcohol function can also be obtained. Molecules containing a three-membered ring and a double bond inside the ring or just close to it cannot be formed. The rate constants for the formation of saturated cycles were close to those presented previously, with an activation energy $1 \mathrm{kcal} / \mathrm{mol}$ higher for the cycles bearing an alcohol function. The rate constants for the formation of an unsaturated cycle (e.g. 2-ethyl-2,5-dihydrofuran) and of a cycle conjugated to a double bond (e.g. 2-vinyl-tetrahydrofuran) are given in table 14. A-factors for the formation of unsaturated cycles $\left(\mathrm{A}_{\text {unsat. }}\right)$ were estimated from those of saturated cycles $\left(\mathrm{A}_{\text {sat. }}\right)$, by considering that an unsaturated free radical contains one less internal rotation than 
the corresponding saturated radical and can then lose one less rotation to give the transition state, i.e. $\log \left(A_{\text {unsat. }}\right)=0.76+\log \left(A_{\text {sat. }}\right)$. Activation energies were estimated from the differences of enthalpies of formation between cycloalkanes and cycloalkenes [29]. As the work of Pedley et al. [217] showed that the strain energy of methylenecyclopentane (5.7 $\mathrm{kcal} / \mathrm{mol}$ ) was very close to that of cyclopentene $(5.9 \mathrm{kcal} / \mathrm{mol}[29])$, the same values have been used for cycles conjugated to a double bond.

\section{TABLE 14}

- For decompositions giving acyclic species, when an enol group (- $\mathrm{C}=\mathrm{C}(\mathrm{OH})-)$ was formed, it was supposed to isomerize instantaneously to give an aldehyde/ketone group $(-\mathrm{CH}-\mathrm{C}(=\mathrm{O})-)$. Table 15 summarizes the rate coefficients specific to the decompositions of hydroperoxyalkenyl radicals; for the other types of reactions, the same values have been used as for hydroperoxyalkyl radicals.

TABLE 15

\section{4.e/ Secondary reactions}

At low and high temperatures, a weak point of the model of Nancy is that no reaction of consumption of dienes has been written. Cyclic ethers with a double bond or with an alcohol function were treated according to the same rules as unsubstituted and saturated cyclic ethers; the formation of acid from cyclic ethers bearing an alcohol function has not been considered. Unsaturated aldehydes were treated according to the same rules as saturated aldehydes, including the abstraction of the aldehydic H-atom followed by a decomposition of the obtained alkoxy radicals.

\section{5/ Conclusion on the modelling of the oxidation of alkenes}

Contrary to alkanes, there are only very few modelling studies of the low temperature oxidation of alkenes representative of those included in gasoline. Only one group has proposed a 
mechanism for 1-hexene and no model has been proposed for the other straight-chain isomers of hexene and for heptenes, despite experimental data having been produced for these compounds. Low temperature models for branched species, even for the smallest one, iso-butene, are missing. Experimental data concerning branched alkenes are also much too scarce, as well as measurements obtained for equivalence ratios outside the 0.4-1 range.

Concerning the existing models of the low temperature oxidation of 1-alkene, while they can satisfactorily enough predict the global reactivity, especially the ignition delay times, they encounter important problems to give an actual repartition of the products: the formation of unsaturated and hydroxy cyclic ethers is overestimated while an important amount of aldehydes is missing. This discrepancy is probably due to the fact that the types of elementary steps and the evaluation of rate constants have been too closely extrapolated from those used for alkanes. Theoretical calculations should allow a better understanding of the reactions channels and to more accurately estimate the related rate parameters. That could be of importance for the addition of $\mathrm{OH} \bullet$ radicals to the double bond, for the reactions of the peroxy radicals deriving from allylic alkenyl radicals, for the isomerization of hydroxyperoxyalkyl radicals (mechanism of Waddington) and for the formation of the new types of cyclic ethers. New experimental measurements on these elementary steps would also be valuable.

\section{4/ CYCLOALKANES}

With the possible increasing importance of fuels derived from non-conventional oil, such as Canadian oil sands [218], the content of cycloalkanes in diesel fuel is expected to increase [219]. That explains why the oxidation of this class of hydrocarbons has been the subject of several studies in the recent years.

\section{1/ Main chemical features of their oxidation}

As for alkanes and alkenes, the first picture of the oxidation of cycloalkanes has been 
drawn by the team of Walker. From 1989, they have studied separate additions of cyclohexane $(753 \mathrm{~K})[220]$ and cyclopentane $(673-783 \mathrm{~K})$ [221] to slowly reacting mixtures of $\mathrm{H}_{2}$ and $\mathrm{O}_{2}$. At about the same time, motivated by industrial safety problems, an experimental study of the oxidation of cyclohexane at $635 \mathrm{~K}$ in a static reactor was performed by the team of Baronnet [222], who proposed the first modelling study of this reaction [223].

These studies have shown that the low temperature chemistry of the oxidation of cycloalkanes is very close to that of alkanes. Figure 21 shows an example of the scheme which is nowadays proposed for cyclohexane. While cycloalkyl radicals also react with oxygen molecules to give cycloalkylperoxyradicals, the yield of formation of the conjugated cycloalkene is larger than for the alkyl radical of the same size under the experimental conditions used by the team of Walker [49]. That is one reason why a cycloalkane always has a larger octane number than the linear alkane of same size; e.g. $\mathrm{RON}$ is 24.8 for $\mathrm{n}$-hexane and 83 for cyclohexane $(\mathrm{MON}=77.2)$ [11]. By successive losses of $\mathrm{H}$-atoms, cyclohexene leads rapidly to the formation of benzene. Isomerizations of cycloalkylperoxyradicals by internal transfer of $\mathrm{H}$-atom are possible, but are hindered by the fact that the involved transition state is bicyclic. Disproportionation between cyclohexylalkoxy radicals was proposed to explain the observed formation of cyclohexanol and cyclohexanone [222]. The reactions of hydroperoxycycloalkyl radicals can produce aldehydes, such as hexenal during the oxidation of cyclohexane, and bicyclic ethers, such as 1,2-epoxycyclopentane, 1,2-epoxycyclohexane or 1,4-epoxycyclohexane [49]. The addition to oxygen of hydroperoxy cycloalkyl radicals can lead to hydroperoxide molecules. This low temperature chemistry of cycloalkanes is in agreement with the existence of the NTC zone during the oxidation of these compounds.

FIGURE 21 
Due to great similarities in their oxidation chemistry between alkanes and cycloalkanes, the three main teams having performed a modelling of the oxidation of cycloalkanes were also those of Livermore, Milano and Nancy.

Up-dating the classes of reactions and the kinetic data proposed for alkanes [70], the team of Westbrook and Pitz has proposed models for the oxidation of cyclohexane (5859 reactions) [224] and methylcyclohexane (7026 reactions) [225]. The team of Ranzi and Faravelli has first proposed a globalized mechanism to model the oxidation of cyclohexane [226], with 2 globalized steps to reproduce the high temperature decomposition and 6 reactions to take into account the low temperature behaviour, but a second more detailed mechanism, with 12 sensitive rate constants estimated through quantum mechanics, has been more recently proposed [227]. The team of Nancy has improved EXGAS software to take into account cyclic reactants [101] and has generated a model for cyclohexane [228] (2446 reactions). Due to the progress made in EXGAS to model the oxidation of alkenes, the low temperature reactions of alkenyl radicals obtained by $\beta$-scission decompositions have been comprehensively considered, but were shown to be mostly of negligible importance.

Table 16 summarizes the little experimental data available for model validation in the case of cycloalkanes. The most studied compound of this family is cyclohexane, which has been studied in rapid compression machines in Lille [229] and in Cambridge (USA) [117], in a jet-stirred reactor by the team of Dagaut (only above $750 \mathrm{~K}$ ) [230-231] and in a pyrex static reactor at low pressure by the team of Baronnet [222]. An important overprediction of the reactivity has been obtained when attempting to model these last results with the model of Nancy. The low temperature oxidation of methylcyclohexane has been studied in two rapid compression machines [117,225] and in a shock tube [232]. The autoignition delay times 
obtained in the rapid compression machine of Galway were well captured by the model of Livermore [225], while those obtained in a shock tube were considerably overestimated. Some measurements have been made in a jet-stirred reactor in Orléans for n-propylcyclohexane [231], but only at temperatures above $950 \mathrm{~K}$. At Drexel University [138], the formation of carbon monoxide has been followed during the oxidation of n-butylcyclohexane in a pressurized flow reactor between 600 and $800 \mathrm{~K}$; these data are still to be modelled. Let us also quote the high temperature $(1160 \mathrm{~K})$ data obtained by Zeppieri et al. [233] for methylcyclohexane in an atmospheric flow tube, as a wide range of products have been analysed. While data have been obtained at high temperature for the autoignition of tricyclodecane (tetrahydrodicyclopentadiene), a component of JP-10 jet fuel [234], there is no published study of the low temperature oxidation of polycyclic alkanes.

\section{TABLE 16}

Figures 22 and 23 show a comparison between the models of Livermore, Milano and Nancy concerning their abilty to reproduce the data of Lille for the autoignition of cyclohexane in a rapid compression machine and for the formation of oxygenated products during the preignition phase, respectively. The three models reproduce well the occurrence of cool flames and of the negative temperature coefficient zone between 725 and $800 \mathrm{~K}$, but the models of Livermore and Milano do not reproduce the formation of a cool flame at $790 \mathrm{~K}$, while the model of Nancy overestimates the ignition delay times between 750 and $850 \mathrm{~K}$. The three models simulate rather correctly the formation of hexenal (not shown in the figure for the model of Milano, but overestimated by a factor 2), which is the main $\mathrm{C}_{6}$ oxygenared products, and of bicyclic ethers. The model of Nancy does not take into account the formation of 1,3-epoxycyclohexane, which was considered as unstable by the team of Walker [220], that of Livermore underestimates the production of 1,2-epoxycyclohexane and that of Milano 
overestimates the formation of 1,4-epoxycyclohexane.

FIGURES 22-23

4.3/ Comparison between the major models in terms of elementary steps and associated rate constants

The chemistry involved in the oxidation of cycloalkanes being close to that proposed for alkanes, the groups of Livermore [224-225], Milano [227] and Nancy [228] have mainly considered the same types of reactions with close kinetic parameters to what is described in paragraph 2.4. We shall then only review here the differences which had to be taken into account due to the presence of the cycle, mainly in the case of isomerizations and the formations of bicyclic ethers.

\section{3.a/ Reactions of cyclo-alkylperoxy radicals}

The presence of the cycle has an important impact on the rate parameters of isomerizations which are presented for the three teams in table 17. The presence of the cycle increases the strain energy of the transition state and the conformational aspects of the cyclic structure disfavours some channels, as the isomerization of cyclohexanylperoxy radicals involving a 7 membered transition state ring, as shown in figure 24 [235].

\section{FIGURE 24}

While in Milano, quantum mechanical calculations were used to determine these rate constants, in Livermore and Nancy, estimations were based on the work of the team of Walker. In Livermore, these parameters have been mainly deduced from the values proposed in their model of iso-octane (see table 8) using corrections obtained by comparison between the values proposed by Handford-Stryring and Walker for cyclohexane [235] and those proposed by Morley and Walker for acyclic alkanes [49] (i.e. A x 0.62, Ea $+0.645 \mathrm{kcal} / \mathrm{mol}$, for a 5 membered transition ring, A x 3.72, Ea $+3.226 \mathrm{kcal} / \mathrm{mol}$, for a 6 membered transition ring, $\mathrm{A} \times$ 
3.47, Ea $+5.305 \mathrm{kcal} / \mathrm{mol}$, for a 7 membered transition ring). This method of correction should be easily applicable to other substituted cyclohexanes. In Nancy, rate parameters were obtained using the method described in paragraph 2.4.b.a. For A-factors, the loss of one rotation was considered in every case; the A-factor of the isomerizations involving a transition state with a 7 members ring was divided by 200 to take into account the ratio of molecules which are in the "boat" form, instead of in the "chair" one (i.e. $0.5 \%$ [49]). While the activation energy for $\mathrm{H}$-abstraction from the substrate by analogous radicals was not changed compared to what is presented in Table 8, the strain energy had to be estimated for bicyclic transition states apart from that of the 4 membered transition ring which was assumed unchanged. The strain energy of the 6 membered transition ring was deduced from the experimental enthalpy of bicyclo[3,3,1]nonane [217]. Those of 5 and 7 membered transition rings were calculated using A-factors, the rate constant of the isomerization involving a 6 membered transition ring and the ratio between the rates of these 3 channels measured by Gulati and Walker at $753 \mathrm{~K}$ [220]. Table 17 shows that the activation energies used by the three research groups for cycloalkanes are much larger than for acyclic compounds (up to $9.5 \mathrm{kcal} / \mathrm{mol}$ difference) leading to lower rate constants. While the 3 models agree with Handford-Styring and Walker [235] that the isomerization involving a 6 membered transition ring is the most favoured, discrepancies are found for the value of this rate constant at $753 \mathrm{~K}$. The values of Livermore and Milano are about two and twenty times larger, respectively, than the values of Nancy and Hull which are similar. This induces important differences between Livermore, Milano and Nancy in the ratio between the constants of the 3 main possible channels. Differences in thermochemical properties which are not reviewed here can partly compensate for these differences in rate constants and explain why the predictions of products are not so far off, as shown in figure 23.

TABLE 17 
The lower reactivity of cyclic alkylperoxy radicals towards isomerizations can lead to an increasing influence of disproportionations. While this class of reaction was not considered in Milano, the disproportionations of alkylperoxy radicals and $\bullet \mathrm{HO}_{2}$ radicals has been taken into account in Livermore and Nancy with the same rate constant as for acyclic alkanes, as well as the disproportionations between two peroxyalkyl radicals, which can be of two kinds as proposed by Lightfoot et al. [167]:

- Disproportionation of two alkylperoxy radicals to give two alkoxy (RO•) radicals, which then react by decomposition, and an oxygen molecule, with $\mathrm{k}=1.4 \times 10^{16} \mathrm{~T}^{-1.62} \exp (-936 / \mathrm{T})$ $\mathrm{cm}^{3} \cdot \mathrm{mol}^{-1} \cdot \mathrm{s}^{-1}$ in Livermore and with $\mathrm{k}=6.3 \times 10^{10} \exp (364 / \mathrm{T}) \mathrm{cm}^{3} \cdot \mathrm{mol}^{-1} \cdot \mathrm{s}^{-1}$ in Nancy (only for cyclic radicals),

- Disproportionation of two alkylperoxy radicals to give an alcohol (e.g. cyclohexanol), a ketone (e.g. cyclohexanone) or an aldehyde and an oxygen molecule; this channel has only been considered in Nancy with $\mathrm{k}=1.4 \times 10^{10} \exp (364 / \mathrm{T}) \mathrm{cm}^{3} \cdot \mathrm{mol}^{-1} \cdot \mathrm{s}^{-1}$.

In the case of cyclohexane, based on the recent work of Carstensen et al. [236] on the reaction of ethyl radicals with oxygen, the teams of Livermore and Milano have also considered the direct elimination of cyclohexene and $\cdot \mathrm{HO}_{2}$ radicals with an A-factor equal to $3.85 \times 10^{12}$ $\mathrm{cm}^{3} \mathrm{~mol}^{-1} \mathrm{~s}^{-1}$ in Livermore and to $7.7 \times 10^{11} \mathrm{~cm}^{3} \mathrm{~mol}^{-1} \mathrm{~s}^{-1}$ in Milano and an activation energy of 29 $\mathrm{kcal} / \mathrm{mol}$. This reaction has not been considered in Nancy, while Silke et al. [224] found ignition delay times to be highly sensitive to the rate expression in use for this reaction. This reaction would not be so important in the case of acyclic peroxy radicals for which the activation energies of isomerizations are lower.

\section{3.b/ Reactions of cyclo-hydroperoxyalkyl radicals}

The presence of the cycle can also modify the kinetics of the decompositions of hydroperoxyalkyl radicals to give bicyclic ethers, the rate parameters of which are given in Table 
18 for the models of cyclohexane of the three teams. In Milano, the rate constants of these decompositions have been calculated by quantum mechanical calculations and the rate parameters used in Livermore have been based on the values proposed in Milano. In Nancy, the activation energies have also been evaluated from quantum mechanical calculations, while A-factor have been mainly based on the changes in the number of losses of internal rotations induced by the formation of the cyclic ether (here one rotation is lost in every case). Important discrepancies between the three teams are also observed here, especially for the formation of 1,2-epoxycyclohexane.

\section{TABLE 18}

For the decompositions of cyclo-hydroperoxyalkyl radicals to give others products, the teams of Livermore and Nancy have mainly used the same classes of reactions and rate parameters as for acyclic compounds. In Milano, quantum mechanical calculations have been used to determine the products obtained and to derive the rate parameters for complex decompositions involving the breaking of several bonds.

\section{3.c/ Reactions of cyclo-hydroperoxyalkylperoxy radicals}

As for acyclic compounds the isomerization/decompositions of hydroperoxyalkylperoxy radicals have been considered. In the model of cyclohexane of Livermore, all the isomerizations have been considered and not only those abstracting an H-atom bound to the atom of carbon bound to an oxygen atom, as was done for acyclic alkanes (see paragraph 2.4.d). The decompositions of the obtained $\bullet \mathrm{U}(\mathrm{OOH})$ radicals have been considered in detail. All the rate parameters for isomerizations have been deduced from the values proposed in their model of iso-octane (see table 8) using the corrections obtained by comparison between the values proposed by Handford-Stryring and Walker for cyclohexane [235] and those proposed by Morley and Walker for acyclic alkanes [49] and applying a reduction of $3 \mathrm{kcal} / \mathrm{mol}$ to the 
activation energy, e.g. for a 5 membered transition state, $\mathrm{A}=0.62 \times 1 \times 10^{11}=6.2 \times 10^{10} \mathrm{~s}^{-1}$ per $\mathrm{H}$-atom and $\mathrm{Ea}=26.850+0.645-3=24.495 \mathrm{kcal} / \mathrm{mol}$. The reduction of $3 \mathrm{kcal} / \mathrm{mol}$ has been proven to have a very large influence on the ignition delay times. In Milano and Nancy, the same rules have been applied as for acyclic compounds, with the rate parameters being derived from those proposed for cyclohexanyl peroxy radicals.

\section{4/ Conclusion on the modelling of the oxidation of cycloalkanes}

Three models of the oxidation of cyclohexane have emerged in the last two years with satisfactory predictions of the experimental data obtained in a rapid compression machine and in a jet-stirred reactor. Unfortunately, there are no data available below $750 \mathrm{~K}$ and in the negative temperature coefficient zone for this last type of apparatus. It is also worth noting that in this recent modelling work, quantum mechanical calculations have been used by several teams to calculate the rate parameters of important channels of the oxidation of cyclohexane Although there is an understanding of the low temperature oxidation of cyclohexane, at least in a range of equivalence ratios limited to $0.5-1.5$, there is no model for the low temperature oxidation of cyclopentane and only one for a substituted cyclo-alkane (methyl cyclohexane). The study of alkyl-cyclohexanes bearing an alkyl chain containing more than one atom of carbon would be interesting, as the lengthening of the substituted alkyl chain would increase the possibilities of isomerization and the complexity of the mechanism. The study of the effect of a second cycle, such as in decalin or tetalin should also be undertaken, as these compounds are found in diesel fuels. In parallel to the development of new models, it will also be fundamental to obtain new experimental data for the oxidation at low temperature for cyclopentane, decalin, tetralin and substituted cycloalkanes (ethyl, propyl, butyl-cyclohexane).

\section{5/ AROMATIC COMPOUNDS}

The phasing out of tetraethyl lead as an anti-knock additive in gasoline has led to an 
increasing content of aromatic compounds, which are now the most important family included in this fuel, as shown in Table 1. This is due to their high octane number, e.g. RON is 120 (MON is 103.5) for toluene and between 116 and 118 for xylenes (MON from 100.0 to 115) [11]. Nevertheless, it is worth mentioning the problems of toxicity involved by the presence of benzene at a concentration above $1 \%$.

\section{1/ Main chemical features of their oxidation}

Due to the presence of the aromatic cycle, the chemistry involved in the oxidation of aromatic compounds strongly differs from that previously described for alkanes, ethers, alkenes and cycloalkanes. Using their experimental results obtained in the Princeton flow tube, the team of Brezinsky has made a pioneering work for the understanding of the mechanism of the oxidation of aromatic hydrocarbons. A review paper has been published in 1986 [237] and has described most of the reaction channels that are still used in the models developed today. These reactions are proposed as a high-temperature mechanism for a temperature range of 875-1500 K, but apart from some alkyl benzenes, there are very few studies concerning aromatic compounds at lower temperatures.

Figure 25 presents the main channels of the oxidation of benzene and toluene as proposed by Brezinsky [237]. Benzene can mainly react by $\mathrm{H}$-abstractions with $\mathrm{OH}$ - radicals to give phenyl radicals or with $\mathrm{O}$-atoms to give phenol or resonance stabilised phenoxy radicals and H-atoms [238]. The main reactions of phenyl radicals considered by Brezinsky were those giving phenoxy radicals, by reaction with $\mathrm{O}$-atoms, $\mathrm{HO}_{2}$ radicals or oxygen molecules. The reaction of phenyl radicals with oxygen molecules giving phenoxy radicals and O-atoms, which is a key step as it is a branching reaction, is not the only possible channel. Other channels can also be considered at low temperature as will be discussed in paragraph 5.4.b. Phenol can react by $\mathrm{H}$-abstraction to give phenoxy radicals, which can decompose to give carbon monoxide and 
cyclopentadienyl radicals, through a two or three-step process [239]. The resonance stabilised cyclopentadienyl radicals react mainly by termination steps to give cyclopentadiene by combination with $\mathrm{H}$-atoms or cyclopentadionyl radicals by reaction with $\cdot \mathrm{HO}_{2}$ radicals or O-atoms. The opening of the cycle occurs by the decomposition of cyclopentadionyl radicals to give butadienyl radicals and carbon monoxide. Only two cyclic compounds were found by the team of Brezinsky in their study of the oxidation of benzene in a flow reactor at $1115 \mathrm{~K}$, namely: phenol and cyclopentadiene [240].

\section{FIGURE 25}

Toluene can mainly react by $\mathrm{H}$-abstractions to give benzyl radicals, with $\mathrm{O}$-atoms to produce cresols or cresoxy radicals [238] or by ipso-addition of $\mathrm{H}$-atoms to form benzene and methyl radicals [241]. Resonance stabilised cresoxy radicals do not have many possibilities of reaction, apart from the combination with $\mathrm{H}$-atoms to give cresols. The fact that benzyl radicals are resonance stabilised has two consequences, first a large number of radicals can be involved in the H-abstractions from toluene and second they mainly react by termination steps. Benzyl alcohol, bibenzyl and ethyl benzyl are produced by combinations between benzyl radicals and $\cdot \mathrm{OH}$ radicals, themselves and methyl radicals, respectively. Benzaldehyde is obtained by reaction of benzyl radicals with $\cdot \mathrm{HO}_{2}$ radicals or with O-atoms and mainly reacts by $\mathrm{H}$-abstraction to give benzoyl radicals, which decomposes easily to yield $\mathrm{CO}$ and phenyl radicals. The additional cyclic compounds found by the team of Brezinsky in their study of the oxidation of toluene in a flow reactor at $1180 \mathrm{~K}$ [242] were benzaldehyde, benzyl alcohol, ethyl benzene, styrene, (ortho, meta, para) cresols and bibenzyl. A study of the team of Walker on the addition of toluene to slowly reacting mixtures of $\mathrm{H}_{2}$ and $\mathrm{O}_{2}$ at $773 \mathrm{~K}$ has allowed them to support the formation of benzylperoxy radicals, its isomerization by internal H-atom transfer and the decomposition to give benzaldehyde and $\mathrm{OH}$ radicals [243]. 
Alkyl benzenes including a side-chain containing more than one carbon atom can react by $\mathrm{H}$-abstractions to give resonance stabilized 1-phenylalkyl radicals, but also other phenylalkyl radicals, with O-atoms to produce alkyl phenols or alkyl phenoxy radicals [238] or by ipsoaddition of $\mathrm{H}$-atoms to form benzene and an alkyl radical including the same number of carbon atoms as the side-chain. At high temperature, phenylalkyl radicals readily decompose by $\beta$-scission to give styrene or substituted styrenes, especially. At low temperature, phenylalkyl radicals can react with oxygen molecules like alkyl radicals, including the formation and the isomerization of peroxy radicals and inducing the formation of phenyl cyclic ethers, alkenylbenzenes and aromatic aldehydes and ketones. For instance, during their study of the oxidation of n-butylbenzene in a rapid compression machine, Roubaud et al. [244] have detected the cyclic ethers shown in figure 26.

\section{FIGURE 26}

Using the same methodology and an extension of the similar types of reactions, the team of Brezinsky has also proposed a mechanism for 1-methylnaphthalene [245].

\section{2/ Detailed chemical models of oxidation}

The two aromatic compounds for which the largest number of models has been developed are the simplest ones, benzene and toluene. Due to the low reactivity of these compounds, there is no real low temperature model for their oxidation. To our knowledge no model of the oxidation of neat benzene or toluene has been satisfactorily validated at temperature below $850 \mathrm{~K}$. Consequently, we will review here models validated in a higher range of temperature than for other hydrocarbons.

The two first detailed models of the oxidation of benzene were those developed in the early 90s by Bittker [246] and Emdee et al. [247] which were both based on the qualitative scheme proposed by Brezinsky [237]. The lowest temperature for which these models were 
validated was $1100 \mathrm{~K}$ using the flow tube data of Lovell et al. [240]. The next models were proposed by Lindstedt and Skevis [248], Zhang and McKinnon [249], Tan and Frank [250], Shandross et al. [251] and Richter et al. [252], but were mainly interested in modelling the results of Bittner and Howard obtained in a low-pressure laminar near-sooting benzene flame [253]. Since 2000, four models have appeared with the aim of modelling the oxidation of benzene in a lower temperature range and were each developed by Alzueta et al. in Lyngby [254], by Ristori et al. in Orléans [255], by Da Costa et al. in Nancy [256] and by SchöbelOstertag et al. in Stuttgart [257]. The lowest temperature for which these models were validated was $850 \mathrm{~K}$. Each of the four teams has modelled its own experimental data obtained in a continuous reactor, which will be described further in this paper. Alzueta et al. [254] and Da Costa et al. [256] have also modelled the results of Lovell et al. [240] in a flow tube.

The two first detailed models of the oxidation of toluene were developed by Emdee et al. [247] and Linsdstedt and Maurice [258]. The lowest temperature for which these models were validated was $1200 \mathrm{~K}$ using the flow tube data of Brezinsky et al. [242]. The model of Emdee et al. was continuously improved by the team of Brezinsky [259-260]. Two recent up-dates of this mechanism have also been made by other teams. The first one was proposed by Andrae et al. [261] in Stockholm (49 species and 229 reactions in a submechanism planned to be used with the model of iso-octane of Livermore [70]) and validated using new shock tube data obtained at high pressure between 1050 and $1250 \mathrm{~K}$ [126]. The second one was mentioned by Chaos et al. [262] in Princeton to model their results obtained in a flow reactor between 850 and $950 \mathrm{~K}$, but was not described in detail. While models for the oxidation of toluene have been recently developed by Djurisic et al. [263] and by Pitz et al. [264] using the data of the team of Brezinsky for their validation in the lowest range of temperatures, two models aiming at being used in a lower temperature range have also been recently proposed by Dagaut et al. (120 species and 920 
reactions) [265] and Bounaceur et al. (128 species and 1036 reactions) [266]. The teams of Orléans and Nancy have validated them at temperatures from $873 \mathrm{~K}$, each using their own jet-stirred reactor data. Bounaceur et al. [266] have also modelled the results of Brezinsky et al. [242] in a flow tube.

Very few models have been developed for monoaromatic compounds heavier than toluene. The team of Orléans has published mechanisms for the oxidation of para-xylene [267], meta-xylene [268] and n-propylbenzene [269] validated at temperatures from $900 \mathrm{~K}$ using their data obtained in a jet-stirred reactor. Mechanisms for the three isomers of xylenes were also proposed by the team of Nancy [270] and were able to well reproduce the difference of reactivity between these three compounds measured by the team of Brezinsky in a flow tube below $1200 \mathrm{~K}$ [271-272], as shown in figure 27. These models did not contain low temperature reactions involving isomerizations of peroxy radicals.

\section{FIGURE 27}

The only real low temperature oxidation model for the oxidation of alkylaromatics heavier than xylene was proposed by Ribaucour et al. [273] to reproduce results obtained for butylbenzene in a rapid compression machine. This model was derived from the mechanism of n-butane extracted from the n-heptane mechanism of Livermore [70] by considering that one $\mathrm{H}$-atom of the n-butane molecule is substituted by an unreactive aromatic nucleus. The particular kinetics associated with benzylic-type radicals, which can be obtained by $\mathrm{H}$-abstractions from the atom of carbon next to the phenyl group, has been added. The full mechanism included 1149 reactions of 197 species, among them 105 aromatic species. This model satisfactorily reproduced experimental cool flame and ignition delay times, as well the selectivity of the main observed products, including the formation of the cyclic ethers shown in figure 26.

As 1-methylnaphthalene can be considered as a model molecule of polyaromatic 
compounds present in diesel fuel, two detailed kinetic mechanisms have been proposed by Pitsch [274] and by Bounaceur et al. [10], respectively. Figure 28 presents the primary reactions taken into account by the team of Nancy based on the experience gained by modelling the oxidation of benzene and toluene and shows that the formation of a wide range of bicyclic aromatic compounds had to be considered.

\section{FIGURE 28}

\section{3/ Experimental results available for validation}

The experimental results concerning the autoignition and the oxidation below $1200 \mathrm{~K}$ of benzene and toluene are presented in Table 19 and those of heavier aromatic compounds in Table 20. Due to the low reactivity of aromatic compounds, the range of investigated temperatures has been extended up to $1200 \mathrm{~K}$ and, as they represent an important part of the available experimental data, papers by the team of Brezinsky published a few years before 1993 have also been quoted.

\section{TABLES 19 - 20}

The only experiental study concerning the ignition of benzene was performed in the rapid compression machine of Cleveland [275]. Apart some results on the ignition of toluene obtained in rapid compression machines in Cambridge (USA) [117], Leeds [111] and Cleveland [275] and in a shock tube in Standford [126] and on that of 1-methylnaphthalene measured in a shock tube in Aachen [127], the main experimental results on the low temperature oxidation of aromatic compounds have been obtained by the team of Minetti in Lille [244-277] in a rapid compression machine between 600 and $900 \mathrm{~K}$. These authors have defined two groups of species. The first group includes toluene, m-xylene, p-xylene and 1,3,5-trimethylbenzene which ignite only above $900 \mathrm{~K}$ and 16 bar. The second group is composed of o-xylene, ethylbenzene, 1,2,3-trimethylbenzene, 1,2,4-trimethylbenzene, n-propylbenzene, 2-ethyltoluene and 
n-butylbenzene, which ignite at much lower temperature and pressure. Results under lean conditions ( $\phi$ from 0.3 to 0.5 ) have been very recently obtained by the same team in the case of n-butylbenzene [282]. Apart from the results on n-butylbenzene from Lille, which were well simulated by Ribaucour et al. [273], and the results on 1-methylnaphthalene from Aachen, which were successfully modelled by Pitsch [274] and by Bounaceur et al. [10], no mechanism able to reproduce satisfactorily low temperature ignition data for aromatic compounds has yet been published. In the case of toluene, the mechanism of Nancy overestimated the delay times obtained in Lille [277] by a factor of about 2, but did predict ignition at pressures below 16 bar [286]. Davidson et al. [126] have shown that the models of Pitz et al. [264] and Dagaut et al. [269] significantly overpredict the data measured for toluene at Standford. Mittal and Sung [275] have tested several models for the oxidation of toluene (Klotz et al. [259], Sivaramakrishnan et al. [260], Djurisic et al. [263], Pitz et al. [264], Bounaceur et al. [266], Dagaut et al. [269]) and have shown that they all fail to reproduce the experimental results obtained in Cleveland.

Even if only temperatures above $900-1000 \mathrm{~K}$ have been studied, the team of Dagaut and that of Brezinsky have made impressive progress by measuring gas chromatographically the profiles of species during the oxidation of a wide range of aromatic compounds in an isothermal jet-stirred reactor and an adiabatic flow-tube, respectively. A recurrent problem in the measurements made in the flow tube used by the team of Brezinsky is the definition of the origin of reaction times. Nevertheless, these results have been used with success by many teams to validate most of the models proposed for benzene, toluene, xylenes and 1-methylnaphthalene. The flow-tube results concerning ethylbenzene, styrene, n-propylbenzene and n-butylbenzene are still to be modelled. The results of Orleans were mainly modelled by the team of Dagaut, but the results of Marchal concerning toluene and 1-methylnaphthalene [278] were successfully modelled by Bounaceur et al. [10,286]. It is worth noting that experimental results concerning 
indane, the simplest naphthenoaromatic hydrocarbon, have been reported in Orléans [285], but not yet modelled.

Five other teams have been recently involved in the study of the slow oxidation of benzene or toluene in continuous reactors at moderate temperature. Chaï and Pfefferle [276] used a micro-jet reactor coupled to a time-of-flight mass spectrometer allowing them to detect a wide range of molecules and radicals during the oxidation of benzene. They have thus analysed the formation of oxygenated species, such as cyclopentadienone (see figure 29a), cyclopentenone, phenoxy radicals, phenol, cyclopentenone and cyclohexenone radicals, benzaldehyde, benzoquinone (see figure 29b) and phenyl peroxide. These results were modelled by Alzueta et al. [254], with important discrepancies concerning phenol, benzoquinone and cyclopentadienone. Alzueta et al. [254] have used a plug flow reactor and followed the consumption of benzene and the formation of carbon oxides, the same apparatus being also used to investigate the pyrolysis and the oxidation of phenol and para-benzoquinone [287]. The team of Nancy [256] has used a jet-stirred reactor with chromatographic analyses to study the oxidation of benzene and toluene, the detected cyclic species being the same as in the work of Brezinsky's team. As relatively high concentrations of reactant were used, significant conversions of benzene or toluene (above $50 \%$ ) were reached around $900 \mathrm{~K}$. In order to reproduce conditions encountered in the cold zones of a waste incinerator, Schöbel-Ostertag et al. [257] have used chromatographic analyses to follow the species profiles in a heated laminar flow reactor in which benzene was injected downstream of the burned gas from a near stoichiometric flame of methane + air. Due to the presence of radicals from the flame entering the reactor, an important conversion of benzene was observed at temperatures as low as $850 \mathrm{~K}$. While the other studies of the oxidation of toluene have been carried out at atmospheric pressure, the team of Dryer in Princeton has worked with a variable pressure flow reactor operated at 12.5 bar, allowing them to also observe a noticeable conversion 
of reactants at temperatures as low as $850 \mathrm{~K}$ [262]. Under these conditions, the consumption of oxygen was underestimated by the models of Dagaut et al. [269], Bounaceur et al. [266] and Sivaramakrishnan et al. [260], confirming the fact that the reaction scheme of this aromatic species is still not well understood at high pressure and low temperature.

\section{FIGURE 29}

5.4/ Comparison between the major models in terms of elementary steps and associated rate constants

As for straight-chain hydrocarbons containing less than three atoms of carbon, it is difficult to describe well-defined types of reactions in the case of the oxidation of monocyclic aromatic compounds. Nevertheless the following part aims at comparing the channels and rate constants used for the additions to the aromatic cycles and the reactions of phenyl, benzyl and cyclopentadienyl radicals by the models proposed to simulate the oxidation of benzene and toluene at the lowest temperatures, i.e. the models of Alzueta et al. [254], Ristori et al. [255], Da Costa et al. [256] and Schöbel-Ostertag et al. [257] for benzene and those of Andrae et al. [261], Dagaut et al. [265] and Bounaceur et al. [266] for toluene. As they have been the basis of many mechanisms in the literature, the reactions proposed by Emdee et al. [247] for the oxidation of benzene, which are included in the mechanism of Andrae et al. [261], will also be taken into account.

\section{4.a/ Additions to the aromatic cycles and derived reactions}

The addition of O-atoms to benzene has been considered by the five models of benzene with a rate constant close to that measured by Nicovich et al. [238] over the temperature range 298-950 K, $\mathrm{k}=2.78 \times 10^{13} \exp (-2470 / \mathrm{T}) \mathrm{cm}^{3} \mathrm{~mol}^{-1} \mathrm{~s}^{-1}$. While Schöbel-Ostertag et al. [257] have considered the formation of phenol; the other models included the formation of phenoxy radicals and $\mathrm{H}$-atoms. The addition of $\mathrm{O}$-atoms to toluene to give cresoxy radicals has been considered 
by Andrae et al. [261] and by Bounaceur et al. [266] with a rate constant close to that proposed by Nicovich et al. [238], $\mathrm{k}=2.56 \times 10^{13} \exp (-1910 / \mathrm{T}) \mathrm{cm}^{3} \mathrm{~mol}^{-1} \mathrm{~s}^{-1}$; this reaction was neglected by Dagaut et al. [265].

The decomposition of phenoxy radicals with the ejection of a carbon monoxide molecule was considered by all models with a rate constant close to that recommended by Baulch et al. [38] of $\mathrm{k}=7.4 \times 10^{11} \exp (-22070 / \mathrm{T}) \mathrm{s}^{-1}$; a similar type of reaction has been assumed for cresoxy radicals by Andrae et al. [261] and by Bounaceur et al. [266]. Terminations have also been proposed for these two resonance stabilized radicals. Combinations with $\mathrm{H}$-atoms to give phenol or cresol have been written in all models. Reactions of phenoxy radicals with O-atoms to give benzoquinone and H-atoms have been considered by Alzueta et al. [254], Da Costa et al. [256] and Schöbel-Ostertag et al. [257]. Alzueta et al. [254] have written the formation of the ortho and para isomers, the other authors considered only one isomer. As proposed by Buth et al. [288], Alzueta et al. [254] and Da Costa et al. [256] have also written the minor channel leading to cyclopentadienyl radicals and carbon dioxide. The disproportionations of phenoxy and cresoxy radicals with $\mathrm{HO}_{2} \bullet$ radicals to give an alcohol and an oxygen molecule, which were only considered in Nancy and in Lyngby, have been shown to have an important retarding effect near $900 \mathrm{~K}$ under jet-stirred reactor conditions [256,266]. A recent theoretical treatment [289] of the reaction of phenoxy and $\mathrm{HO}_{2} \bullet$ radicals has shown the formation of phenoxy-OOH adducts with the $\mathrm{OOH}$ group in ortho or para positions. These adducts decompose to give mainly phenol and oxygen molecules or benzoquinones and water. While there is some consensus between the different teams on the main reactions of formation and consumption of phenoxy radicals, the reactions of cresoxy radicals are still uncertain and would need additional studies.

While the addition of $\mathrm{H}$-atoms to benzene to give cyclohexadienyl radicals has only been considered by Da Costa et al. [256], the addition of H-atoms to toluene to give benzene and 
methyl radicals have been written in the three models with a rate constant close to that recommended by Baulch et al. [38] of $\mathrm{k}=5.8 \times 10^{13} \exp (-4070 / \mathrm{T}) \mathrm{cm}^{3} \mathrm{~mol}^{-1} \mathrm{~s}^{-1}$.

The additions of $\cdot \mathrm{OH}$ radicals to benzene and toluene to give phenol and cresol, respectively, have only been considered by the team of Nancy. It is worth noting that this addition followed by the addition to oxygen of the adduct mainly controls the chemistry of the decomposition of aromatic compounds under atmospheric conditions [290]. One could wonder if such reactions, which are similar to those considered for alkenes, could be of importance during the oxidation of benzene and toluene at much lower temperatures than what has been investigated during the validation of the present models. Such conditions could be met when studying the oxidation of mixtures of an aromatic compound and a more reactive hydrocarbon.

\section{4.b/ Reactions of phenyl radicals}

While phenyl radicals can react by additions to unsaturated molecules (e.g. Dagaut et al. [265] have considered additions to $\mathrm{C}_{2} \mathrm{H}_{2}, \mathrm{C}_{2} \mathrm{H}_{4}, \mathrm{C}_{4} \mathrm{H}_{2}, \mathrm{C}_{4} \mathrm{H}_{4}$ ) or by combinations with some radicals (e.g. Da Costa et al. [256] have considered combinations with O-atoms, $\bullet O H$, methyl, $\mathrm{HCO} \bullet$, vinyl, ethyl, $\mathrm{HO}_{2} \bullet$, propargyl and phenyl radicals), a rate of reaction analysis at $923 \mathrm{~K}$ under the jet-stirred reactor conditions [256] has shown that the dominant consumption channels of phenyl radicals were with oxygen molecules. The five models have considered the branching reaction involving the formation of phenoxy radicals and O-atoms with a rate constant close to that proposed by Frank et al. [291], $\mathrm{k}=2.6 \times 10^{13} \exp (-3080 / \mathrm{T}) \mathrm{cm}^{3} \mathrm{~mol}^{-1} \mathrm{~s}^{-1}$. The models of Alzueta et al. [254], Da Costa et al. [256] and Schöbel-Ostertag et al. [257] also included the formation of benzoquinone and $\mathrm{H}$-atoms with a rate constant proposed by Frank et al. [291], $\mathrm{k}=$ $3.0 \times 10^{13} \exp (-4520 / \mathrm{T}) \mathrm{cm}^{3} \mathrm{~mol}^{-1} \mathrm{~s}^{-1}$. A doubt remains about the structure of the molecule of benzoquinone obtained. Frank et al. [291] and Schöbel-Ostertag et al. [257] considered parabenzoquinone and Alzueta et al. [254] and Da Costa et al. [256] the ortho-isomer. A mechanism 
for the oxidation of para-benzoquinone was developed by Alzueta et al. [287]. To reproduce their experimental results at $923 \mathrm{~K}$, Da Costa et al. [256] have also considered the formation of phenylperoxy radicals with a rate constant estimated to $\mathrm{k}=3.2 \times 10^{19} \mathrm{~T}^{-2.5} \mathrm{~cm}^{3} \mathrm{~mol}^{-1} \mathrm{~s}^{-1}$, in agreement with the value measured by $\mathrm{Yu}$ and Lin [292] at $473 \mathrm{~K}$ and the decomposition of these peroxy radicals to give o-benzoquinone and $\mathrm{H}$-atoms or cyclopentadienone and $\mathrm{HCO}$ • radicals.

A more theoretical treatment of the reaction of phenyl with oxygen molecules has been undertaken by several teams. Recent quantum mechanical calculations have been performed by Bozzelli et al. [293-294], Sirjean et al. [295] and Tokmakov et al. [296] by using GAUSSIAN [297]. To illustrate the obtained results, figure 30 presents the reaction mechanisms leading to o-benzoquinone and cyclopentadienone as calculated by Sirjean et al. [295]. The rate constants proposed by Bozzelli et al. [293] were incorporated in the mechanism for toluene proposed by Pitz et al. [264]. The most complete scheme was computed by Tokmakov et al. [296], who proposed as major products cyclopentadienyl radical + carbon dioxide, pyranyl + carbon monoxide, o-benzoquinone $+\mathrm{H}$, and 2-oxo-2,3-dihydrofuran-4-yl $\left(\mathrm{C}_{4} \mathrm{OH} \mathrm{H}_{3} \mathrm{O}\right)+$ acetylene. While the presence of benzoquinone and cyclopentadienone has been detected by Chaï and Pfefferle [275], no evidence of the formation of products deriving from pyranyl and oxodihydrofuranyl radicals has been found in the different experimental studies of the oxidation of benzene published in the literature.

\section{FIGURE 30}

\section{4.c/ Reactions of benzyl radicals}

At low temperature, the main reactions of resonance stabilized benzyl radicals are termination steps and addition to oxygen molecules. The termination with $\mathrm{HO}_{2} \bullet$ radicals has been shown to have an important promoting effect at $893 \mathrm{~K}$ under jet-stirred reactor conditions 
[266]. The teams of Stockholm [261] and Orléans [265] have considered the direct formation of benzyl alkoxy and $\cdot \mathrm{OH}$ radicals, while the team of Nancy [266] has written the formation of benzyl hydroperoxide molecules, which further decompose to give the same products; theoretical calculations by Davis et al. [298] have shown that the distinction between the two channels becomes academic above $800-900 \mathrm{~K}$. The three teams have used a rate constant close to that proposed by Hippler et al. [299] over the temperature range of $1200-1500 \mathrm{~K}, \mathrm{k}=5 \times 10^{12}$ $\mathrm{cm}^{3} \mathrm{~mol}^{-1} \mathrm{~s}^{-1}$, and have written (i) the decomposition of benzoxy radicals to give benzaldehyde and H-atoms (enthalpy of reaction of $15.9 \mathrm{kcal} / \mathrm{mol}$ ). The teams of Nancy and Stockholm have also considered (ii) the formation of formaldehyde and phenyl radicals (enthalpy of reaction of $23.6 \mathrm{kcal} / \mathrm{mol}$ ). In Nancy, the same rate was taken into account for both channels, $\mathrm{k}_{\mathrm{i}}=\mathrm{k}_{\mathrm{ii}}=$ $2 \times 10^{13} \exp (13900 / \mathrm{T}) \mathrm{s}^{-1}$. In Stockholm, the following values were used: $\mathrm{k}_{\mathrm{i}}=1.3 \times 10^{14} \exp (550 / \mathrm{T})$ $\mathrm{s}^{-1}$ as proposed by Brezinsky et al. [242] (the same value as in Orléans) and $\mathrm{k}_{\mathrm{ii}}=4 \times 10^{13}$ $\exp (1010 / \mathrm{T}) \mathrm{s}^{-1}$. This important discrepancy between the rate constants used in the different models and the lack of consistency with the enthalpies of reaction make new experiments or calculations giving more information about the decomposition of benzoxy radicals particularly needed.

At low temperature, the other important combination takes place between benzyl radicals themselves to produce bibenzyl, but was not taken into account by Dagaut et al. [265]. Andrae et al. [261] and Bounaceur et al. [266] have used the rate constant proposed by Müller-Markgraf and Troe [300] over the temperature range 300-1500 K, $\mathrm{k}=2.51 \times 10^{11} \mathrm{~T}^{0.4} \mathrm{~cm}^{3} \mathrm{~mol}^{-1} \mathrm{~s}^{-1}$. The terminations with methyl and $\cdot \mathrm{OH}$ radicals to give ethyl benzene and benzylalcohol, respectively, are of minor importance.

The reactions of benzyl radicals with oxygen molecules were not of great influence under jet-stirred reactor conditions [266], but they were much more important during the preignition 
phase in a shock tube [261], as they can be a source of reactive radicals. Andrae et al. [261] have written two branching steps (i) the formation of O-atoms, phenyl radicals and formaldehyde and, a minor channel, (ii) the production of O-atoms and benzoxy radicals, with the following rate constants, $\mathrm{k}_{\mathrm{i}}=6.32 \times 10^{11} \exp (7300 / \mathrm{T}) \mathrm{cm}^{3} \mathrm{~mol}^{-1} \mathrm{~s}^{-1}$ and $\mathrm{k}_{\mathrm{ii}}=6.32 \times 10^{12} \exp (21600 / \mathrm{T}) \mathrm{cm}^{3} \mathrm{~mol}^{-1} \mathrm{~s}^{-1}$ as proposed by Brezinsky et al. [242]. As stated by the authors [261], $\mathrm{k}_{\mathrm{i}}$ has been tuned to match shock tube data for neat toluene [126] and for n-heptane/toluene mixtures [301]. Dagaut et al. [265] have also written (iii) the formation of benzaldehyde and $\bullet \mathrm{OH}$ radicals, with $\mathrm{k}_{\mathrm{iii}}=\mathrm{k}_{\mathrm{ii}}$. Bounaceur et al. [266] have considered reaction (ii) with the rate constant proposed by Brezinsky et al. [242]. The idea of a fourth channel, (iv) the formation of benzyl hydroperoxy radicals, which can isomerise and decompose to give benzaldehyde and $\bullet \mathrm{OH}$ radicals, is supported by experimental results of Ellis et al. [243] and theoretical calculations of Clothier et al. [302]. This sequence of reactions, which was taken into account by Lindstedt and Maurice [258], was included in the model of Nancy with the rate constant for the addition to oxygen molecule proposed by Fenter et al. [303] over the temperature range $298-398 \mathrm{~K}, \mathrm{k}_{\mathrm{iv}}=4.6 \times 10^{11} \exp (190 / \mathrm{T})$ $\mathrm{cm}^{3} \mathrm{~mol}^{-1} \mathrm{~s}^{-1}$. The rate parameters for the isomerization were calculated using the method described in paragraphs 2.4.B. $\alpha$ and 3.4.c, $\mathrm{k}_{\text {Nancy }}=3.4 \times 10^{9} \mathrm{~T} \exp (-18900 / \mathrm{T}) \mathrm{s}^{-1}$. This rate constant, which was not a sensitive parameter under the conditions of Bounaceur et al. [266], was much lower than that proposed by Ellis et al. [243] at $773 \mathrm{~K}\left(\mathrm{k}_{\text {Nancy }}=6.3 \times 10^{1} \mathrm{~s}^{-1}\right.$ instead of $\mathrm{k}_{\text {Hull }}=2.8 \times 10^{3} \mathrm{~s}^{-1}$ ), with a much higher activation energy than that calculated by Clothier et al. $[302](\mathrm{Ea} / \mathrm{R}=14570 \mathrm{~K})$.

More investigations about the rate constant of the reactions of benzyl radicals with $\mathrm{HO}_{2} \bullet$ radicals and oxygen molecules are greatly needed to better model the oxidation of toluene at low temperature and high pressure. 


\section{4.d/ Reactions of cyclopentadienyl radicals}

Zhong and Bozzelli [304] have theoretically investigated the reactions of resonance stabilized cyclopentadienyl radicals with oxygen radicals, hydrogen and oxygen atoms and $\bullet \mathrm{OH}$ and $\cdot \mathrm{HO}_{2}$ radicals. The combinations with $\mathrm{H}$-atoms to produce cyclopentadiene have been considered in all models with a rate constant close to that proposed by Allara et al. [171] for that type of reaction, $\mathrm{k}=1 \times 10^{14} \mathrm{~cm}^{3} \mathrm{~mol}^{-1} \mathrm{~s}^{-1}$. Dagaut et al. [265] have considered its pressure dependence. But the main reactions at low temperature are with $\bullet \mathrm{HO}_{2}$ radicals and oxygen molecules. This last reaction was not taken into account in Orléans and Princeton. In Nancy and Lyngby, it was considered to produce (i) vinyl ketene $\left(\mathrm{CH}_{2}=\mathrm{CHCH}=\mathrm{C}=\mathrm{O}\right)$ and $\cdot \mathrm{HCO}$ radicals, with the rate constant calculated by Zhong and Bozzelli [304] at 1 bar over the temperature range $900-1300 \mathrm{~K}, \mathrm{k}_{\mathrm{i}}=1.2 \times 10^{19} \mathrm{~T}^{-2.48} \exp (-5520 / \mathrm{T}) \mathrm{cm}^{3} \mathrm{~mol}^{-1} \mathrm{~s}^{-1}$. In Stuttgart the formation of (ii) cyclopentadienone and $\cdot \mathrm{OH}$ radicals was written with a rate constant, $\mathrm{k}_{\mathrm{ii}}=2.3 \times 10^{12}$ $\exp (-18228 / \mathrm{T}) \mathrm{cm}^{3} \mathrm{~mol}^{-1} \mathrm{~s}^{-1}$ [257]. The calculations of Zhong and Bozzelli [304] have shown the ratio $\mathrm{k}_{\mathrm{ii}} / \mathrm{k}_{\mathrm{i}}$ to be equal to $1 / 5000$ at $900 \mathrm{~K}$. Apart from that of Schöbel-Ostertag et al. [257], all the models have considered the combinations of cyclopentadienyl and $\cdot \mathrm{HO}_{2}$ radicals leading to cyclopentadionyl and $\mathrm{OH}$ radicals. Alzueta et al. [254] have used the rate constant calculated by Zhong and Bozzelli [304], $\mathrm{k}=6.3 \times 10^{29} \mathrm{~T}^{-4.69} \exp (-5863 / \mathrm{T}) \mathrm{cm}^{3} \mathrm{~mol}^{-1} \mathrm{~s}^{-1}\left(\mathrm{k}=1.3 \times 10^{13} \mathrm{~cm}^{3} \mathrm{~mol}^{-1} \mathrm{~s}^{-1}\right.$ at $900 \mathrm{~K}$ ), the other teams have used estimated values, $\mathrm{k}=3 \times 10^{12} \mathrm{~cm}^{3} \mathrm{~mol}^{-1} \mathrm{~s}^{-1}$ in Nancy [256] and $\mathrm{k}=3 \times 10^{13} \mathrm{~cm}^{3} \mathrm{~mol}^{-1} \mathrm{~s}^{-1}$ in Princeton and in Orléans [247, 255]. It is worth noting that the reactions of consumption of vinyl ketene, cyclopentadione and cyclopentadionyl radicals are still very uncertain.

Two other combinations should be quoted as they lead to the formation of aromatic compounds: the combinations with methyl radicals, which is a source of benzene, and that with cyclopentadienyl radicals, which can produce naphthalene. Lifshitz et al. [305] have studied the 
thermal reaction of methylcyclopentadiene, including the formation of benzene, which takes place only from radical intermediates that have a methylene group connected to the five-membered ring. Melius et al. [306] have calculated the potential energy surface for the formation of naphthalene from two cyclopentadienyl radicals and have proposed that it occurs in two steps: the formation of $\mathrm{H}$-atoms and dihydrofulvalenyl $\left(\cdot \mathrm{C}_{10} \mathrm{H}_{9}\right)$ radicals followed by the decomposition of these radicals to give naphthalene and $\mathrm{H}$-atoms. While this channel has been proven to be important under flame conditions [307], the formation of dihydrofulvalene $\left(\mathrm{C}_{10} \mathrm{H}_{10}\right.$, see figure 29c) should be also envisaged at low temperature [256,308].

\section{5/ Conclusion on the modelling of the oxidation of aromatic compounds}

While numerous validated models have been proposed for the oxidation of pure benzene and toluene above $850 \mathrm{~K}$, there is a complete lack of such mechanisms for lower temperatures. That is related to a deficit in experimental data under these conditions because of the low reactivity of these aromatic species. Autoignition data for toluene are not well simulated below $900 \mathrm{~K}$, mainly due to problems in the understanding of the reactions of benzyl radicals. Experimental data obtained for mixtures of benzene and toluene with more reactive hydrocarbons should be used to test these models below $900 \mathrm{~K}$. Low temperature (600-900 K) ignition data have been published for xylenes, ethyl benzene, trimethylbenzenes, ethyltoluenes and butyl-benzene, but this last compound is the only alkylbenzene, the low temperature oxidation of which has been modelled. Even high temperature validated models are relatively scarce for alkylaromatics, detailed mechanisms being only available for xylenes, n-propylbenzene and 1-methylnaphthalene. Intermediate temperature (950-1350 K) oxidation data have been obtained for a naphthenoaromatic compound, indane, but have not yet been modelled. Low temperature data for the ignition of this last compound would also be very valuable. As for the other families of compounds reviewed in the previous parts of this paper, 
there is a need for experimental data to validate the models in an extended range of equivalence ratios, i.e. below 0.5 and above 3 .

The extension of the validity of the models for the oxidation of aromatic compounds towards lower temperatures will require more experimental and theoretical information about some potentially important elementary steps under these conditions: the addition of $\bullet \mathrm{OH}$ radicals to aromatic rings, the reactions between phenylic-type (such as phenyl, naphthyl, etc) and benzylic-type (such as benzyl, 1-phenyl-1-alkyl, phenylbenzyl, etc) radicals with $\cdot \mathrm{HO}_{2}$ radicals or oxygen molecules, the reactions of oxy-aromatic (such as phenoxy, cresoxy, naphthoxy) or alkoxy-aromatic (such as benzoxy) radicals. For polyalkylbenzenes such as xylenes, the kinetics of the isomerization involving the transfer of a H-atom from one branch to another one in peroxy radicals [244] should be investigated. A better understanding of the chemistry of $\mathrm{C}_{5}$ oxygenated compounds, such as cyclopentadienone, would also be of interest.

\section{6/ APPLICATIONS TO SURROGATE MIXTURES}

The procedure for the development of chemical surrogates for gasoline and diesel fuel can certainly be inspired from the strategy well stated by Ranzi et al. [309-310] in two papers describing the formulation of a JP-8 surrogate fuel. This strategy was the following:

"1. Feasibility. Candidates in the formula must have known detailed kinetic mechanisms.

2. Simplicity. Mainly limited for computational capabilities to normal paraffins with less than 12 carbons, monocyclic paraffins with less than 8 carbons, and simple aromatics such as benzene, alkyl-benzenes and naphthalene.

3. Similarity. The surrogate is required to match practical fuels on both physical and chemical properties: (i) volatility (boiling range and flash point), (ii) sooting tendency (smoking point and luminous number), (iii) combustion property (heat of combustion, flammability limits and laminar premixed mass burning rate). 
4. Cost and availability."

Of course, in the case of gasoline surrogates, branched paraffins and alkenes should also be taken into account. In the case of diesel surrogates, the presence of parraffins including more than 12 atoms of carbons and naphtheno-aromatics might be envisaged. For the development of HCCI engines, the autoignition properties of surrogates are also of particular importance. Two recent papers written by authors from various US laboratories have assessed the current state of research concerning surrogates for gasoline [311] and diesel fuel [312] and outline the main development needed for the related experimental database and modelling tools.

\section{1/ Main features about reaction coupling}

There are two main theories concerning the interactions between fuels during the oxidation of mixtures. The first one, which was taken as a basis by Klotz et al. [259] during their study of the oxidation of toluene/butane blend at $1200 \mathrm{~K}$, postulated that chemical interactions were limited to an effect on the common pool of free radicals. That means that retarding influence of the addition of toluene during alkane oxidation would be mainly due to a decrease of the concentrations of small radicals, such as $\mathrm{H}$ - and $\mathrm{O}$-atoms and $\bullet \mathrm{OH}$ and $\bullet \mathrm{HO}_{2}$ radicals, which can be trapped by $\mathrm{H}$-abstractions from toluene to give unreactive benzyl radicals. The second theory considers "cross term" reactions to account for a possible fuel interactivity. A pioneering study of the low temperature oxidation of alkanes mixtures was performed by Westbrook, Warnatz and Pitz in 1988 [313] for iso-octane/n-heptane mixtures It proposed "cross term" reactions between heptyl radicals and iso-octane and between iso-octyl radicals and n-heptane, but found them of little influence because the concentrations of alkyl radicals were small. A wide range of "cross term" reactions have been used in more recent models, e.g. H-abstractions from a parent fuel $(\mathrm{RH})$ by phenyl, benzyl and $\mathrm{R}^{\prime} \mathrm{O}_{2} \bullet$ radicals which are derived from another R'H parent fuel, and will be detailed further in this paper. An experimental 
demonstration of the occurrence of "cross term" reactions has been given by Vanhove et al. [314]. They have detected, during the preignition phase of a 1-hexene/toluene mixture, the formation of 1-buten-3yl-benzene and 1-hepten-3yl-benzene which can only be obtained by combinations of radicals deriving from both fuels (benzyl radicals from toluene and allyl and 1hexene-3-yl radicals from 1-hexene).

\section{2/ Detailed chemical models of the low temperature oxidation}

As the model of a mixture is based on the combination of models for each of its components, the main groups active in modelling the oxidation of mixtures are those who have already worked on models of neat fuels, i.e. mainly the teams of Livermore, Milano, Nancy and Stockholm.

Normal-heptane and iso-octane are primary reference fuels (PRF) for octane rating in spark-ignited internal combustion engines; their octane numbers are 0 and 100, respectively. Reference fuels, which have intermediate octane numbers, are obtained from mixtures of these two compounds and are used to measure the octane number of actual gasoline in CFR engines. For a given octane number, the modelling of the reference mixture is important to understand the chemical phenomena which govern the autoignition of relevant gasoline. That explains why the n-heptane/iso-octane blend (PRF mixture) has long been the most studied mixture.

In Livermore, based on the classes of reactions and the estimations of rate expressions defined to model the oxidation of n-heptane [70] (see paragraph 2.2.a), a model for the low temperature oxidation of PRF mixtures [315] has been written and validated using experimental data obtained in a flow reactor [136] and in a shock tube [119]. The more recent model for the oxidation of iso-octane [70] included that for $n$-heptane and can then be used to model the oxidation of PRF mixtures. In this model, the chemical interactions between n-heptane and iso-octane were only induced by an action on the common pool of free radicals. Still more 
recently, the team of Westbrook and Pitz has published a model for a surrogate for gasoline containing n-heptane, iso-octane, 1-pentene, toluene and methylcyclohexane [316-317]. This model was based on the mechanism for the low temperature oxidation PRF mixtures [70], which initially included high temperature reactions for 1-pentene. Their recent models for toluene [264] (see paragraph 5.2) and methylcyclohexane [225] (see paragraph 4.2), which are previously described in this paper, as well as "cross term" reactions, were added to produce a global mechanism consisting of 1214 species and 5401 reactions. This model was used to reproduce satisfactorily experimental data obtained in a HCCI engine for mixtures of these five components and in a shock tube for n-heptane/iso-octane/toluene blends [121].

In Stockholm, a model for the low temperature oxidation of PRF mixtures was proposed based on that of Livermore [70], but including additional 131 "cross term" reactions, and validated on experimental data obtained in a shock tube [119], a rapid compression machine [117] and the measurements of the authors in a HCCI engine [318]. At the same time, a model for the oxidation of n-heptane/toluene blends was obtained by merging the model for toluene oxidation of Dagaut et al. [265] (see paragraph 5.2) and the newest version for n-heptane of Livermore [70] and by adding 12 "cross term" reactions. This model was used to reproduce data obtained in the same HCCI engine [318]. In the more recent paper describing their model for the oxidation of toluene, Andrae et al. [261] have also presented a new model for the oxidation of PRF/toluene mixtures, which was obtained by merging the model of Livermore for PRF mixtures [70] with their new model for toluene written for this study (see paragraph 5.2) and by adding 167 "cross term" reactions. This model (1083 species in 4535 reactions) was used with success to reproduce the data obtained in shock tubes at high pressure for n-heptane/toluene blends [301] and for PRF/toluene mixtures [121].

Three other models have been derived from the work of Livermore. Dubreuil et al. [22] 
from Orléans have satisfactorily modelled their results obtained in a jet-stirred reactor for the oxidation of the n-heptane/toluene blend with a mechanism made by merging that of Livermore for n-heptane [70] and that of Nancy for toluene [266] (see paragraph 5.2). No "cross term" reaction was described. Vanhove et al. [319] in Lille have simulated their own results obtained in a rapid compression machine for binary and tertiary mixtures containing iso-octane, 1-hexene and toluene [314]. This tertiary mixture can be considered as a good gasoline surrogate candidate. They have used the mechanism of Livermore for iso-octane [70], that of Nancy for 1-hexene [186] and an in-house one for toluene. "Cross term" reactions were mentioned: $\mathrm{H}$-abstractions between peroxyalkyl or hydroxyperoxyalkyl radicals and iso-octane or 1-hexene molecules and $\mathrm{H}$-abstractions from toluene by allylic alkenyl radicals. Chaos et al. [262] in Princeton have coupled a minimized model derived from the PRF mechanism of Livermore [70] with their recent one for toluene written for this study (see paragraph 5.2). They have used it to simulate their own results obtained in a flow reactor, as well as measurements made for PRF [119], n-heptane/toluene [301] and PRF/toluene [121] mixtures in shock tubes. No "cross term" reactions were considered.

In Milano, a semi-detailed model for the low-oxidation of PRF mixtures was obtained by merging their models for n-heptane [96] and iso-octane [97] (see paragraph 2.2.b). This model [136], involving about 150 species and 3000 reactions, was validated using experimental data obtained in a flow reactor in Princeton, in a rapid compression machine in Lille and in a jet-stirred reactor in Orléans [320]. By adding their mechanisms for MTBE and ETBE (see paragraph 2.2.b) to their model of n-heptane, the group of Ranzi and Faravelli has been able to reproduce the retarding effect of the addition of these ethers on the oxidation of n-heptane in a jet-stirred reactor [92], as was experimentally measured in Orléans [321]. Based on their semidetailed model for the low temperature oxidation of n-dodecane [92], which already contains 
reactions for simple aromatic compounds (see paragraph 2.2.b), on a semi-detailed sub-mechanism for the low-oxidation of iso-cetane $(2,2,4,4,6,8,8$-heptamethylnonane) and on high temperature lumped reactions for methylcyclohexane, models for the oxidation of n-dodecane/iso-cetane, n-dodecane/methylcyclohexane and n-dodecane/1-methylnaphthalene blends have been proposed [140-141]. The validation of the models for these binary mixtures, which can be considered as surrogates for diesel fuel, was performed using experimental data obtained in a flow reactor. A model for a surrogate of kerosene containing n-dodecane, isooctane, methylcyclohexane, benzene and toluene has been written [309] and validated under flame conditions [322]. Models for still more complex fuels have been obtained by adding high temperature lumped reactions for n-tetradecane and decalin [310]. Only the "cross term" reactions involving the $\mathrm{H}$-abstraction from the parent fuel by isobutyl and isobutenyl radical, which come from the iso-octane decomposition, were systematically considered.

In Nancy, a model for the low temperature oxidation of PRF mixtures was generated using EXGAS software (see paragraph 2.2.b). The first version [323] was validated using data obtained in a jet-stirred reactor [320] and an improved version (529 species and 2477 reactions) [24] was generated to model ignition data obtained in a rapid compression machine [136] and in a shock tube [119]. No "cross term" reaction was taken into account. In order to test a surrogate for gasoline, Pires da Cruz et al. [324] have merged this last model for PRF mixtures with that for the oxidation of toluene previously developed in Nancy [266] (see paragraph 5.2), with the addition of 21 “cross term" reactions. Validation for n-heptane/toluene blends was performed using experimental results obtained in a rapid compression machine [314], a jet-stirred reactor [22] and a HCCI engine [318]. Simulations for iso-octane/toluene mixtures were made in order to reproduce data measured in a rapid compression machine [314] and a HCCI engine [318]. Tests for PRF/toluene blends were made against data obtained in a shock tube [119] and in a 
rapid compression machine [117]. The reactivity of n-heptane/toluene was underestimated at low temperature (below $800 \mathrm{~K}$ ) and the rate constant of the decomposition of $\mathrm{H}_{2} \mathrm{O}_{2}$ molecules needed to by increased by a factor of 4 in order to fit results above 40 bar. For the PRF/toluene blends in a rapid compression machine, figure 31 shows that the sensitivity of the reactivity to small variations of the iso-octane to n-heptane ratio with a fixed amount of toluene is well captured by the mechanism [324], although the simulated ignition delay differences between fuels are smaller than the experimental ones [117]. A surrogate for diesel fuel has also been investigated: a mechanism for the oxidation of n-decane/1-methylnaphtalene mixtures [10] has been obtained by merging the low temperature mechanism for n-decane, as generated by EXGAS software, with that for 1-methylnaphtalene developed in the same study, with the addition of the two types of “cross term" reactions shown in figure 32. Qualitative validation was made against experimental autoignition delay times measured in a pressurized chamber at 3 bar in which a suspended droplet was suddenly brought to a temperature from $600 \mathrm{~K}$ to $750 \mathrm{~K}$ [149]. Models for n-heptane/MTBE and n-heptane/ETBE blends [107] were also generated by using EXGAS software and successfully tested against the experimental results obtained in a jet-stirred reactor [321]; "cross term" reactions were neglected. The new version of EXGAS designed to generate models for the oxidation of alkenes (see paragraph 3.2) was used to produce a mechanism for an iso-octane/1-hexene blend [325], which was satisfactorily used to model data obtained in a rapid compression machine [314]; for this last mechanism, "cross term" reactions were automatically generated.

\section{FIGURES 31- 32}

Böhm et al. [79] have qualitatively reproduced their experimental results obtained in a static reactor [54] for n-pentane/ether mixtures by incorporating their models for ethers in the n-heptane oxidation mechanism of Chevalier et al. [85] (see paragraph 2.2.a). No "cross term" 
reactions were mentioned.

\section{3/ Experimental results available for validation}

The experimental results concerning the autoignition and the oxidation below $900 \mathrm{~K}$ of mixtures of compounds containing more than 4 atoms of carbon are shown in Tables 21 to 23 . The two types of mixtures the most often investigated are binary mixtures containing either PRF (Table 21) or an alkane and an aromatic compound (Table 22). In the mid 90s, due to the interest in octane improvers, few studies have been focused on binary mixtures of an alkane and an ether molecules (Table 21). In the five last years, binary mixtures (Table 22), as well as ternary and quaternary blends (Table 23), containing an alkene molecule, have also been investigated. Only two studies of Drexel University have dealt with blends containing a cyclo-alkane.

\section{TABLES 21-23}

Concerning autoignition, the rapid compression machine of Lille was used to study an impressive range of blends containing PRF [136], 1-hexene, benzene or toluene [314], but not the PRF/toluene mixture, which has often been proposed as a valuable gasoline surrogate candidate. The machine of Cambridge (USA) has been used to investigate PRF, n-heptane/toluene and PRF/toluene blends [117] and that of Cleveland in the case of toluene/diisobutylene (2,4,4-trimethyl-2-pentene) and iso-octane/toluene mixtures [328]. Results in shock tubes are less abundant. The high-pressure shock tube of Aachen was used to study PRF mixtures [121], measurements concerning PRF/toluene mixtures were made in Standford [119] and the apparatus of Duisburg was used to investigate PRF mixtures [326], n-heptane/toluene [301] and n-heptane/toluene/iso-octane/di-iso-butylene blends [330]. The two recent studies concerning di-iso-butylene, which has a similar structure to iso-octane, are of particular interest due to the great lack of results concerning branched alkenes.

Apart from the results obtained in the flow reactor of Princeton by the team of Dryer for 
the oxidation of PRF mixtures [136] and PRF/toluene blends [262] and those published by the team of D'Anna of Napoli for the oxidation of n-heptane/toluene mixtures in a jet-stirred reactor [134], most data obtained in a continuous flow reactor for the oxidation of mixtures were obtained by the team of Dagaut in Orléans in an isothermal jet-stirred reactor $[22,320$ $321,327,329]$ and by the team of Cernansky in a flow reactor [138-141]. Mainly focused on the search of gasoline surrogates, the team of Dagaut has studied PRF mixtures [320], mixtures of n-heptane with ethers [321] and with toluene [22] and blends containing iso-octane, 1-hexene or toluene, including a quaternary iso-octane /1-hexene/ETBE/toluene mixture [327,329]; a high temperature model has been proposed by this team to reproduce these last results. While the other studies have been only performed from $800 \mathrm{~K}$, the oxidation of PRF, n-heptane/MTBE, n-heptane/ETBE and n-heptane/toluene mixtures has been investigated from $550 \mathrm{~K}$, i.e. including the complete NTC zone, and has shown that the addition of iso-octane, ethers and toluene has a strong retarding effect on the reactivity of n-heptane. Mainly interested in the surrogates of diesel and jet fuels, the team of Cernansky [138-141] has studied the oxidation of n-decane/iso-octane, n-dodecane/iso-cetane, n-dodecane/methylcyclohexane and n-dodecane/ 1-methylnaphthalene binary mixtures and of tertiary blends, containing n-decane, n-butylcyclohexane or methylcyclohexane and n-butylbenzene or toluene, using the control cool down method (CDD), in which the reactor was stabilized to a specified maximum temperature and then allowed to cool at a fixed rate and a constant pressure and residence time. The results concerning tertiary blends are still to be modelled

A study of the effect of the addition of TAME, MTBE, ETBE on the induction periods of the cool flames of n-pentane oxidation has been carried out by the team of Baronnet [54] in a static reactor at low pressure for very rich mixtures. The strong non-linear increase of the induction period with the added amount of ether well demonstrates the anti-knock effect of these 
compounds.

6.4/ Comparison between the major models in terms of elementary steps and associated rate constants

The types of considered "cross term" reactions depend on the nature of the mixtures. We will distinguish "cross term" reactions for alkane mixtures, for alkane/alkene blends and for mixtures containing an aromatic compound. The rate parameters used by the three teams for the reactions involving peroxy radicals or species deriving from toluene are summarized in Tables 24 and 25, respectively. The "cross term" reactions involving $\mathrm{H}$-abstraction from alkanes, cycloalkanes and alkenes by alkyl and alkenyl radicals are in most cases negligible below $900 \mathrm{~K}$ and are therefore not detailed here.

\section{TABLES 24-25}

\section{4.a/ Mixtures of alkanes}

"Cross term" reactions for alkane mixtures have been considered in Stockholm [126], Livermore [316-317] and Nancy [103]. In Stockholm, "cross term" reactions involved in the oxidation of PRF mixtures have been comprehensively taken into account [126]. They have written all the $\mathrm{H}$-abstractions from n-heptane by iso-octyl and iso-octylperoxy radicals and from iso-octane by linear or branched heptyl and heptylperoxy radicals; the reactions of branched $\mathrm{C}_{7}$ radicals are not really "cross term" reactions, as these species are derived from iso-octane. The users of the mechanisms of Stockholm should be aware that the rate coefficients for "cross term" reactions have been reevaluated between the first [318] and the second [126] papers. The more realistic rate coefficients used in the last paper lead to a strong decrease of the influence of these reactions.

In the model of Livermore for a surrogate of gasoline [317], some "cross term" reactions between PRF that were missing in their first mechanism [70] and that were proposed by Andrae 
et al. [318] were included using rate coefficients modified to be consistent with their previous rate rules [317]. The H-abstractions from n-heptane, iso-octane and methylcyclohexane by small alkylperoxy radicals were considered, as well as reactions between alkylperoxy radicals for various alkyl groups of different fuel components. "Cross term" reactions between the species of the base PRF mechanism and methylcyclohexane were also incorporated.

In Nancy, the software EXGAS is designed to consider systematically the "cross-term" reactions: each time a radical is created, it is submitted to all the possible generic propagations without considering its reactant of origin. That means that "cross term" reactions involving $\mathrm{H}$-abstractions from parent a fuel $(\mathrm{RH})$ by $\mathrm{R}^{\prime} \bullet$ and $\mathrm{R}^{\prime} \mathrm{O}_{2} \bullet$ radicals could be taken into account on request [104]. But these reactions have been shown to have a negligible influence in the case of alkane blends and their generation was usually not performed. The rate parameters for alkylperoxy radicals, which were close to those used in Livermore and Stockholm, were taken from Chevalier et al. [84].

\section{4.b/ Alkane/alkene blends}

The oxidation of alkane/alkene mixtures has only been modelled in Livermore [316-317] and Nancy [325]. In Livermore, the H-abstractions from 1-pentene by small alkylperoxy radicals and from n-heptane, iso-octane and methylcyclohexane by 1-penten-3-yl radicals have been written.

In the Nancy model for iso-octane/1-hexene blends, two types of "cross term" reactions have been generated and found to be of negligible importance [325]:

- The H-abstractions from iso-octane or 1-hexene by peroxy radicals.

In the mechanisms concerning a single class of hydrocarbon, only the peroxy radicals deriving directly from the reactant (i.e. those which can be obtained through only 2 elementary steps: an $\mathrm{H}$-abstraction or an addition of $\mathrm{OH}$ to the double bond followed by an addition to an oxygen 
molecule) can react by $\mathrm{H}$-abstraction. Applying this rule in the present case, only iso-octyl peroxy $\left(\mathrm{C}_{8} \mathrm{H}_{17} \mathrm{OO} \bullet\right)$, 1-hexenylperoxy $\left(\mathrm{C}_{6} \mathrm{H}_{11} \mathrm{OO} \bullet\right)$ or hydroxyhexylperoxy $\left(\mathrm{C}_{6} \mathrm{H}_{12} \mathrm{OHOO} \bullet\right)$ radicals were involved.

- The combinations of the allylic radicals deriving from 1-hexene, 1-hexen-3-yl radicals and 3-hexen-2-yl radicals, with $\mathrm{tC}_{4} \mathrm{H}_{9}$ and $\mathrm{iC}_{3} \mathrm{H}_{7}$ radicals, with a rate constant $\mathrm{k}=4.2 \times 10^{12}$ $\mathrm{cm}^{3} \mathrm{~mol}^{-1} \mathrm{~s}^{-1}$

Amongst the radicals obtained from the decompositions by beta-scission of iso-octyl or derived radicals, only $\mathrm{tC}_{4} \mathrm{H}_{9}$ and $\mathrm{iC}_{3} \mathrm{H}_{7}$ radicals were taken into account for combinations because they cannot decompose by beta-scission involving the breaking of a C-C bond and are consequently less reactive than other radicals.

\section{4.c/ Mixtures containing an aromatic compound}

"Cross term" reactions for mixtures containing an aromatic compound have been considered, in Stockholm [126], Livermore [316-317] and Nancy [10-324]. The three teams have considered the H-abstractions from toluene by alkyl and alkylperoxy radicals, with notable differences in the used rate constants in the case of alkylperoxy radicals. In Nancy, only the $\mathrm{H}$-abstraction from toluene with methylperoxy radicals has been found of importance [324]. The teams of Stockholm [126] and Livermore [316-317] have also written the H-abstractions from benzaldehyde by peroxyalkyl radicals and the Stockholm team [126] has further written H-abstractions from PFRs by phenyl radicals. The team of Livermore [316-317] has taken into account the H-abstractions from 1-pentene by 1-penten-3-yl radicals and from 1-pentene and methylcyclohexane by benzyl radicals. Finally, the team of Nancy [324] has also considered the $\mathrm{H}$-abstractions from toluene by resonance stabilized iso-butenyl, iso-octenyl and heptenyl radicals and the combinations between benzyl and alkyl radicals. In the case of the n-decane/1-methylnaphthalene blends [10], the H-abstractions from n-decane by phenylbenzyl 
radicals have been written, as well as the combinations between phenylbenzyl and decyl radicals (see figure 32), this last reaction having the same rate constant as that with benzyl radicals.

\section{5/ Conclusion on the modelling of the oxidation of mixtures}

While kinetic mechanisms for the oxidation of gasoline surrogates have been first restricted to PRF, these few last years have seen the emergence of numerous models for a widening range of mixtures, as well as an important production of experimental data to validate them, even if, as for pure fuels, the range of investigated equivalence ratios would need to be extended. This effort has been motivated by the strong interest of the automotive industry in the development of HCCI engines.

The studies concerning gasoline surrogates have been focused towards blends made of an alkane mixed with an alkene or an aromatic compound, mainly toluene, and towards mixtures containing more than two components. Several models are now available for the oxidation of PRF/toluene mixture, but they suffer from the uncertainties in the mechanism of the oxidation of toluene at low temperature and high pressure, e.g. in Stockholm, the rate constant of the reaction of benzyl radicals and oxygen molecules had to be tuned in order to get satisfactory results, while in Nancy, an arbitrary variation of the A-factor of the decomposition of $\mathrm{H}_{2} \mathrm{O}_{2}$ molecules was made. A five component surrogate (PRF/toluene/methylcyclohexane/1-pentene) has even been proposed, but with high temperature chemistry for the alkene oxidation.

Models for diesel fuel surrogates have also just appeared, e.g. n-dodecane/iso-cetane or n-dodecane/1-methylnaphthalene, even if the experimental data available for their validation are still very scarce.

In mixtures containing alkanes or alkenes, the proposed "cross term" reactions have been found to be of negligible importance when cross effects occur quasi-exclusively via the radicals pool. For mixtures containing toluene, H-abstractions from toluene, as well as combinations 
involving benzyl radicals could be of some importance and may at least explain the formation of minor products, as shown by the experimental work of Vanhove et al. [314].

\section{8/ CONCLUSION}

This review has assessed the recent gas-phase detailed kinetic models developed to simulate the low temperature (below $900 \mathrm{~K}$ ) oxidation and autoignition of the components of gasoline and diesel fuel (alkanes, ethers, esters, alkenes, cycloalkanes and aromatics, each including 4 or more atoms of carbon) and of the mixtures of several of them, which have been proposed as surrogates for actual fuels.

Concerning pure compounds, this analysis has shown that numerous models have been proposed for alkanes, with a wide range of experimental data available to validate them. This abundance of models diminishes when other families of components are considered. Branched compounds and those containing more than ten carbon atoms have been far less thoroughly studied. For alkenes containing more than five carbon atoms, only the reactions of straight-chain 1-pentene and 1-hexene have been modelled, and discrepancies with experimental results for the formation of the products were obtained. There is no model for the low temperature oxidation of branched alkenes. Models have been proposed for cyclohexane and methylcyclohexane, but there are no simulations concerning cyclopentane or other substituted cycloalkanes. Important problems remain in the modelling of the oxidation of aromatic compounds, especially at low temperature and high pressure. This is particularly true for toluene, which is frequently considered in gasoline surrogates. Experimental and modelling studies are also missing for naphtheno-aromatic compounds. The range of validity of models in term of equivalence ratio is narrow and close to stoichiometry. Validated modelling for equivalence ratios below 0.5 , which can be of importance in HCCI engines, and above 3, which can be observed in some parts of the combustion chamber of diesel engines, are very scarce, combined with a lack of experimental 
data. Kinetic models can qualitatively explain the differences in RON between various compounds. However the observed variations of sensitivity (the difference between RON and MON) with the type of fuel are still not well understood, e.g. sensitivity is far larger for alkenes than for alkanes

The literature is relatively rich in detailed kinetic models for the oxidation of mixtures of components of gasolines, but far poorer for species included in diesel fuel. These models can in most cases satisfactorily capture the evolution of autoignition delay times with temperature and pressure, and the formation of the major products (carbon oxides, ethylene). Validated models for multicomponent mixtures representative of gasoline (e.g. n-heptane/iso-octane/toluene or isooctane/1-hexene//toluene blends) and to a lesser extent diesel fuel, are starting to become available. The question about the optimum number of components which should be considered in a surrogate for it to be well representative of an actual fuel, without increasing unreasonably the size of the model, is still open. It is certainly important that the components of a surrogate are carefully chosen so that they are well representative of the species present in the actual fuel. Models for each component of the surrogate should first be well defined separately and validated on a wide range of experimental conditions. In most types of mixtures, "cross term" reactions have been found to be negligible. Co-oxidation between reactants occurs quasi-exclusively via the radical pool. For mixtures containing aromatic compounds, "cross term" reactions involving resonance stabilized radicals could be of some importance, at least to explain the formation of minor products.

In the case of both pure compounds and multicomponent mixtures, it is important that the experimental database will still be increasing, with results obtained in systems, which are physically well characterised, such as rapid compression machines, shock tubes, jet-stirred or flow reactors. More detailed measurements of reaction products would also be of help for a 
better understanding of the reaction channels. The development of new techniques allowing the measurement of the concentration of radical species (e.g. $\mathrm{HO}_{2} \bullet$ radicals) in these apparatus would also be of great interest.

As it is important that no determining reaction is omitted, the development of detailed kinetic models mainly involves the inclusion of a comprehensive reactions base and the systematic writing of relevant elementary steps according to given rules. This necessary method of development means that low temperature models include hundreds of species and thousands of reactions, which hinders their use for practical applications and even prohibits their incorporation into a reactive flow solver, even for 1D computation. However, the fact that detailed models are based on elementary steps should also allow them to be used in a predictive way to give clues in cases where experimental investigations are difficult. Comprehensive models are also of interest for a better identification of the elementary steps which govern combustion reactions.

When analysing major models of the literature in term of the considered types of elementary steps and in the used rate coefficients, it is striking to see the important differences existing even for simple compounds such as alkanes. The differences in kinetic data are certainly also related to differences in thermochemical properties. That explains why similar agreement with experimental data can be observed when comparing simulations using various models. These differences would however probably lead to important deviations between models when trying to model the formation of minor products, such as heavy alkenes, dienes, aromatic compounds, aldehydes, ketones, alcohols and acids, which can be toxic to human health and are of great importance in air pollution, as they are involved in the formation of urban smog and acid rain. If a satisfactory prediction of the formation of these species is desired, the degree of accuracy of the models should be increased and fundamental studies on the involved elementary 
steps would be needed, including experimental work and theoretical calculations. The parameterizing of the rate constants as a function of temperature and pressure (P-T parameterization) should also be used for an increasing range of multi-channel reactions, e.g. the reactions of oxygen molecules with phenyl radicals or alkyl radicals containing more than four atoms of carbon. The mechanism of the degradation of the first oxidation products, such as hydroperoxides, alkenes, cyclic ethers and aldehydes, needs also to be better understood.

New determinations of rate constants, based on both recent experimental techniques and calculations using quantum mechanical and master equation methods, should certainly lead to a reduction of the existing uncertainties. That would be of particular interest for the following elementary steps which are important in low temperature oxidation models:

- The reactions with oxygen molecules of alkyl, alkenyl, allylic and benzylic radicals including more than four carbon atoms, with a careful P-T parameterization for the concurrent channels, e.g. the formation of peroxy radicals and that of alkenes and $\cdot \mathrm{HO}_{2}$ radicals.

- The formation of cyclic ethers including bicyclic compounds, with a characterisation of the possible products in the case of alkenes.

- The reactions of large allylic and derived radicals, which are taken at the moment to be those of allyl radicals.

- The reactions with $\cdot \mathrm{HO}_{2}$ radicals, especially with allylic and benzylic radicals.

- The additions of $\bullet \mathrm{OH}$ radical to a double bond or to a phenyl ring.

- The low temperature reactions of cyclic compounds, with a determination of the influence of the presence of an alkyl chain or of a strained cycle.

- The reactions of oxy radicals, especially during the oxidation of aromatic species (e.g. phenoxy and cresoxy radicals). 
More accurate determinations of rate constants will hopefully allow a better agreement to be obtained between the models which will be developed in the future. Nevertheless, differences will certainly remain due to the manner in which these models are developed. If we consider the groups of Livermore, Milano and Nancy, which have produced the biggest part of the models quoted in this paper, they each have a specific way of doing it. The "hand-made" work of Livermore has led to the thoroughly validated mechanisms for a few key reactants (up to $\mathrm{C}_{8}$ ) and mixtures of them which are important in gasoline. At Milano, the coupling of automatic generation with a clever work of lumping has allowed them to study several compounds, as well as mixtures, representative of those included in diesel fuels. The completely automated method used in Nancy was well suited to propose models for alkenes, the complexity of their chemistry making manual and semi-manual methods difficult to be used. This last system could also be of interest to rapidly write mechanisms for alkanes or alkenes never investigated before or to systematically test changes in generic reactions and rate constants. With these three systems being rather complementary, it could be tempting to amalgamate the best pieces from each of them. That could certainly be of interest as shown by Dubreuil et al. [22], who have coupled the mechanism of n-heptane of Livermore [70] with that of toluene of Nancy [266]. Thist should however be carefully done, as the models produced by each system are self-consistent, i.e. in each group, the thermochemical data, the reaction base for small species and the primary and secondary mechanisms have been tailored to work together.

The uncertainties in the rate constants of the key reactions of importance in low temperature oxidation mechanisms can certainly be still reduced by a large factor after additional studies. However, unavoidable errors on these parameters would probably always limit the possible accuracy which can be reached. An optimization procedure, as that used in the GRIMech database for the oxidation of methane [40], could certainly be extended to the case of 
heavier hydrocarbons, but it is feared that this procedure, which changes some parameters compared to their average recommended value, would lead to large discrepancies when leaving the range of conditions used for the optimization and would then hinder the use of the obtained models in a predictive way. Nevertheless, detailed kinetic models can only gain to be validated on the widest possible range of experimental conditions. That involves a better organisation of the collaborations between model developers and (experimental and theoretical) data providers, preferentially at an international level. The sharing of experimental data and models, such as that proposed in the PrIMe (Process Informatics Models) network described by Frenklach [331], should be strongly encouraged. The development of kinetic mechanisms should also be more and more seen as a part of a multiscale approach, which needs theoretical data obtained at the quantum level, but also aims at providing models to be used in engines simulations by oil and automotive industrial researchers.

Their large size explains why an important effort has been made for several years to reduce detailed schemes. The methods of reduction have been well reviewed in 1997 by Tomlin et al. [81] and summarized in 2007 by Law [332]. The majority of the methods developed for mechanism reduction falls into two categories, that is, skeletal reduction that eliminates unimportant species and reactions, and time-scale reduction that moderates the stiffness. A first method of the skeletal reduction is the elimination of unimportant species and reactions using sensitivity analysis, principal components analysis [333] or rate of reaction analysis. The detailed mechanisms of Livermore for iso-octane (860 species) [70] has been reduced by Lu and Law [334] and Saylam et al. [335] to skeletal mechanisms consisting of 233 and 300 species, respectively. Automated procedures have been applied by Porter et al. [336] to reduce the reaction mechanism of Nancy describing the oxidation of cyclohexane [228] from 499 species to 60 necessary species. The level of reduction is a function of the modelling abilities that are 
sought to be kept in the reduced model: the skeletal model of Saylam et al. [335] is still relatively large, but it is able to represent the formation of heavy alkenes and cyclic ethers.

A second method is the lumping of different isomers into a single species, e.g. the four heptylperoxy radicals are represented by $\mathrm{C}_{7} \mathrm{H}_{15} \mathrm{OO} \bullet$ or $\mathrm{ROO} \bullet$. This method has been widely and successfully used in Milano by Ranzi et al. [92] to obtain semi-detailed mechanisms, as well as tested in Nancy [337]. As it cannot be applied to the reactions of small species, this method does not allow very important reduction factors for reactants containing less than 10 atoms of carbon. Nevertheless, it is well suited to reduce primary or secondary mechanisms and can be of great interest when treating species of large size, such as those included in diesel fuel [93], or mixtures of several compounds.

The methods of reduction based on the time-scales involved in the kinetic equations include the quasi-steady-state approximation (QSSA) [338], the computational singular perturbation (CSP) method [339] and the low-dimensional manifold (ILDM) method [340]. Few applications of these techniques to model the low temperature oxidation of large hydrocarbons have been published. By introducing QSSA, Soyhan et al. [341] have reduced a skeletal n-heptane/iso-octane mechanism (63 species and 386 reactions) deriving from the work of Chevalier [84] to 19 species and 16 global reactions. By using the CSP method from the detailed mechanisms of Livermore for n-heptane (561 species) [70], Valorani et al. [342] have obtained a skeletal mechanism consisting of 124 species.

Detailed or reduced models can also be used to run large numbers of simulations in order to derive fitted functions or repro-models (mostly polynomials) [338, 343] or look-up tables in order to store target data under a wide range of conditions (pressure, temperature, mixture compositions) and to use them to run reactive flow simulations. Look-up tables, including chemical progress rate, cool flame ignition delay time and heat release [344] or mole fractions of 
major species [345], have been recently built from the mechanisms of Nancy for n-heptane and n-decane/1-methylnaphthalene blends. They have been used satisfactorily to run real engine CFD 3D computations and to obtain the results shown in figure 1 [10].

\section{ACKNOWLEDGEMENTS}

This review work has greatly benefited from the experience gained with the team developing EXGAS software, and especially with Dr. Valérie Warth, Prof. Guy-Marie Côme, Prof Gérard Scachi, Prof. René Fournet, Dr. Pierre-Alexandre Glaude, Dr. Roda Bounaceur and Dr. Olivier Herbinet. The author wishes to thank particularly Dr. François Baronnet for his helpful advices in the writing of this paper, as well as Dr. William J. Pitz for sending the mechanism for gasoline surrogate of Naik et al. [317], Dr. Charles K. Westbrook for providing the original of a figure and Dr. Richard Porter for improving the written English of this paper. 


\section{REFERENCES}

[1] Westbrook CK, Mizobuchi Y, Poinsot TJ, Smith PJ, Warnatz J. Computational combustion. Proc Combust Inst 2005;30:125-57.

[2] Litzinger TA. A review of experimental studies of knock chemistry in engines. Prog Energy Combust Sci 1990;162:155-167.

[3] Liberman M A, Ivanov MF, Peil OE, Valiev DM, Eriksson L-E. Numerical modeling of the propagating flame and knock occurrence in spark-ignition engines. Combust Sci and Tech 2005; 177:151-82.

[4] Mehl M, Faravelli T, Ranzi E, Lucchini T, Onorati A, Giavazzi F et al. Kinetic modeling of knock properties in internal combustion engines. 2006;SAE 2006-01-3239.

[5] McEnally CS, Pfefferle LD, Atakan B, Kohse-Höinghaus K. Studies of aromatic hydrocarbon formation mechanisms in flames: Progress towards closing the fuel gap. Prog Energy Combust Sci 2006;32:247-94.

[6] Richter H, Howard JB. Formation of polycyclic aromatic hydrocarbons and their growth to soot - a review of chemical reaction pathways. Prog Energy Combust Sci 2000;26:565608.

[7] Aceves SM, Flowers DL, Martinez-Frias J, Smith JR, Westbrook CK, Pitz WJ et al. A Sequential Fluid-Mechanic Chemical-Kinetic Model of Propane HCCI Combustion. 2001;SAE 2001-01-1027.

[8] Results of the review of the Community Stategy to reduce $\mathrm{CO}_{2}$ emissions from passenger cars and light-commercial vehicles. Communication from the commission to the council and the European Parliament. Commission of the European Communities, Brussels, 2007

[9] Kalghatgi GT. Sustainable automotive fuels for the future. Proceedings of the $3^{\text {rd }}$ European Combustion Meeting, Chania, 2007. 
[10] Bounaceur R, Glaude PA, Fournet R, Battin-Leclerc F, Jay S, Pires da Cruz A. Kinetic modelling of a surrogate diesel fuel applied to 3D auto-ignition in HCCI engines. Int $\mathrm{J}$ Vehicle Design 2007;44:124-42.

[11] Guibet JC. Fuels and engines. Paris, Publications de l'Institut Français du Pétrole, Editions Technip, 1999.

[12] Agarwal AK. Biofuels (alcohols and biodiesel) applications as fuels for internal combustion engines. Prog Energy Combust Sci 2007;33:233-71.

[13] Graboski MS, McCornick RL. Combustion of fat and vegatable oil derived fuels in diesel engines. Prog Energy Combust Sci 1998;24:125-64.

[14] Westbrook CK, Pitz WJ, Curran HJ. Chemical kinetic modeling of the effects of oxygenated hydrocarbons on soot emissions from diesel engines. J Phys Chem A 2006;110:6912-22.

[15] Jacobson MZ. Effect of ethanol (E85) versus gasoline vehicles on cancer mortality in the United States. Environ Sci Technol 2007;41:4150-57.

[16] Bünger J, Krahl J, Baum K, Schröder O, Müller M, Westphal G, Ruhnau P, Schulz TG, Hallier E. Cytotoxic and mutagenic effects, particle size and concentration analysis of diesel engine emissions using biodiesel and petro diesel as fuel. Arch Toxicol 2000;74:490-98.

[17] Tomlin AS. Can combustion technologies reduce our impact on both the global climate and local air quality? Proceedings of the $3^{\text {rd }}$ European Combustion Meeting, Chania, 2007.

[18] Simmie JM. Detailed chemical kinetic models for the combustion of hydrocarbon fuels. Prog Energy Combust Sci 2003;29:599-634.

[19] Frassoldati A, Faravelli T, Ranzi E. Kinetic modeling of the interactions between NO and hydrocarbons at low temperature. Combust Flame 2003;132:188-207. 
[20] Frassoldati A, Faravelli T, Ranzi E. Kinetic modeling of the interactions between NO and hydrocarbons at high temperature. Combust Flame 2003;135:97-112.

[21] Moréac G, Dagaut P, Roesler JF, Cathonnet M. Nitric oxide interactions with hydrocarbon oxidation in a jet-stirred reactor at 10 atm. Combust Flame 2006;145:512-20.

[22] Dubreuil A, Foucher F, Mounaïm-Rousselle C, Dayma G, Gagaut P. HCCI combustion: effect of NO in EGR. Proc Combust Inst 2007;31:2879-86.

[23] Griffiths JF. Reduced kinetic models and their application to practical combustion systems. Prog Energy Combust Sci 1995;21:25-107.

[24] Buda F, Bounaceur R, Warth V, Glaude PA, Fournet R, Battin-Leclerc F. Progress towards an unified detailed kinetic model for the autoignition of alkanes from $\mathrm{C}_{4}$ to $\mathrm{C}_{10}$ between 600 and 1200 K. Combust Flame 2005;142:170-86. http://www.ensic.inplnancy.fr/DCPR/Anglais/GCR/exgas_distribution.htm.

[25] Burcat A, Ruscic B. Third millennium ideal gas and consensed phase thermochemical database for combustion with updates from active thermochemical tables. Argonne National Laboratory report 2005. http://garfield.chem.elte.hu/Burcat/burcat.html .

[26] Allendorf MD. HiTempThermo database. 2006. http://www.ca.sandia.gov/HiTempThermo/index.html.

[27] Ritter ER, Bozzelli JW. THERM: thermodynamic property estimation for gas phase radicals and molecules. Int J Chem Kinet 1991;23:767-78.

[28] Muller C, Michel V, Scacchi G, Côme GM. A computer program for the evaluation of thermochemical data of molecules and free radicals in the gas phase. J Chim Phys 1995;92:1154-77. http://www.ensic.inpl-nancy.fr/DCPR/.

[29] Benson SW. Thermochemical Kinetics. $2^{\text {nd }}$ ed., John Wiley, New York, 1976.

[30] Lissianski VV, Zamansky VM, Gardiner WC. Combustion chemistry modeling. In Gas- 
phase combustion chemistry, Gardiner WC Ed., Springer-Verlag, New York, 2000.

[31] Tsang W, Hampson RF. Chemical kinetic data base for combustion chemistry. Part 1. Methane and related compounds. J Phys Chem Ref Data, 1986;15:1087-279.

[32] Tsang W. Chemical kinetic data base for combustion chemistry. Part 2. Methanol. J Phys Chem Ref Data 1987;16:471-508.

[33] Tsang W. Chemical kinetic data base for combustion chemistry. Part 3. Propane. J Phys Chem Ref Data 1988;17:887-951.

[34] Tsang W. Chemical kinetic data base for combustion chemistry. Part 4. Isobutane. J Phys Chem Ref Data 1990;19:1-68.

[35] Tsang W. Chemical kinetic data base for combustion chemistry. Part 5. Propene. J Phys Chem Ref Data 1991;20:221-73.

[36] Baulch DL, Cobos CJ, Cox RA, Esser C, Franck P, Just Th et al. CEC group on evaluation of kinetic data for combustion modeling. J Phys Chem Ref Data 1992;21:411.

[37] Baulch DL, Cobos CJ, Cox RA, Franck P, Hayman GD, Just Th et al. CEC group on evaluation of kinetic data for combustion modeling. J Phys Chem Ref Data 1994;23:847.

[38] Baulch DL, Bowman CT, Cobos CJ, Cox RA, Just Th, Kerr JA et al. Evaluated kinetic data for combustion modeling: supplement II. J Phys Chem Ref Data 2005;34:757-1397.

[39] Troe J. Fall-off curves of unimolecular reaction. Ber Buns Phys Chem 1974;78:478-88.

[40] Smith GP, Golden DM, Frenklach M, Moriarty NW, Eiteneer B, Goldenberg M, et al. http://www.me.berkeley.edu/gri mech/

[41] Konnov AA. Detailed reaction mechanism for small hydrocarbons combustion. Release 0.5. 2000. http://homepages.vub.ac.be/ akonnov.

[42] Tan Y, Dagaut P, Cathonnet M, Boettner JC. Oxidation and ignition of methane-propane and methane-ethane-propane mixtures: experiments and modelling. Combust Sci and 
Tech 1994;103:133-51.

[43] Hughes KJ, Turányi T, Clague AR, Pilling MJ. Development and testing of a comprehensive chemical mechanism for the oxidation of methane. Int $\mathrm{J}$ Chem Kinet 2001;33:513-38. http://www.chem.leeds.ac.uk/Combustion/Combustion.html.

[44] Kee RJ, Rupley FM, Miller JA. Chemkin II. A fortran chemical kinetics package for the analysis of a gas-phase chemical kinetics. Sandia Laboratories Report, SAND 89-8009B 1993. http://www.reactiondesign.com/.

[45] Knox JH. Photochemistry and reaction kinetics. Ashmore PG, Sugden TM, Dainton FS Ed., Cambridge University Press, Cambridge, 1967.

[46] Fish A. Oxidation of organic compounds, vol 2. Advances in Chemistry Series, 76;1968:69.

[47] Pollard RT. Hydrocarbons. In Comprehensive chemical kinetics: gas-phase combustion. Bamford CH, Tipper CFH Eds, 17, Elsevier, Amsterdam, 1977.

[48] Cox RA, Cole JA. Chemical aspects of autoignition of hydrocarbon-air mixtures. Combust Flame 1985;60:109-23.

[49] Walker RW, Morley C. Basic chemistry of combustion. In Comprehensive Chemical Kinetics: low-temperature combustion and autoignition, Pilling MJ Ed., 35, Elsevier, Amsterdam, 1997.

[50] Griffiths JF, Mohamed C. Experimental and numerical studies of oxidation chemistry and spontaneous ignition phenomena. In Comprehensive Chemical Kinetics: low-temperature combustion and autoignition, Pilling MJ Ed., 35, Elsevier, Amsterdam, 1997.

[51] Westbrook CK, Dryer FL. Chemical kinetic modelling of hydrocarbon combustion. Prog Energy Combust Sci 1984;10:1-57.

[52] Warnatz J. The mechanism of high temperature combustion of propane and butane. 
Combust Sci and Tech 1983;34:177-200.

[53] Brocard JC, Baronnet F, O'Neal HE. Chemical kinetics of the oxidation of methyl tertbutyl ether (MTBE). Combust Flame 1983;52:25-35.

[54] El Kadi B, Baronnet F. Study of the oxidation of unsymmetrical ethers (ETBE, TAME) and tentative interpretation of their high octane numbers. J Chim Phys 1995;92:706-25.

[55] Fischer SL, Curran HJ, Dryer FL. The reaction kinetics of dimethyl ether. I: hightemperature pyrolysis and oxidation in flow reactors. Int J Chem Kin 2000; 32:713-40.

[56] Curran HJ, Fischer SL, Dryer FL. The reaction kinetics of dimethyl ether. II: lowtemperature oxidation in flow reactors. Int J Chem Kin 2000;32:741-59.

[57] Choo KY, Golden DM, Benson SW. Very low-pressure pyrolysis (VLPP) of t-butylmethyl ether. Int J Chem Kin 1974;6:631-41.

[58] Brocard JC, Baronnet F. Effets de parois dans la pyrolyse du methyl tert-butyl eyher (MTBE). J Chim Phys 1987;84:19-25.

[59] Demirbas A. Biodiesel production from vegatable oils via catalytic and non-catalytic supercritical transesterification methods. Prog Energ Combust Sci 2005;31:466-87.

[60] Baronnet F, Brocard JC. Cool flames and molecular structure of organic compounds. Oxidat Commun 1983;4:83-95.

[61] Dagaut P, Gail S, Sahasrabudhe M. Rapeseed oil methyl ester oxidation over extended ranges of pressure, temperature and equivalence ratio: Experimental and modeling kinetic study. Proc Combust Inst 2007;31:2955-61.

[62] Halstead MP, Kirsch LJ, Prothero A, Quinn CP. A mathematical model for hydrocarbon ignition at high pressure. Proc R Soc Lond A 1975;346:51-5.

[63] $\mathrm{Hu} \mathrm{H}$, Keck JC. Autoignition of adiabatically compressed combustible gas mixtures. 1987;SAE 96:872210. 
[64] Tanaka S, Ayala F, Keck J. A reduces chemical kinetic model for HCCI combustion of primary reference fuels in a rapid compression machine. Combust Flame 2003;133:467-81.

[65] Pitz WJ, Wilk RD, Westbrook CK, Cernansky NP. The oxidation of n-butane at low and intermediate temperatures: an experimental and modeling study. Paper $n^{\circ}$ WSSCI 88-51, Western States Sections / The Combustion Institute Spring Meeting, 1988.

[66] Ribaucour M, Minetti R, Sochet LR, Curran HJ, Pitz WJ, Westbrook CK. Ignition of isomers of pentane: an experimental and kinetic modeling study. Proc Combust Inst 2000;28:1671-78.

[67] Curran HJ, Pitz WJ, Westbrook CK, Hisham MWM, Walker RW. An intermediate temperature modeling study of the combustion of neopentane. Proc Combust Inst 1996;26:641-49.

[68] Wang S, Miller DL, Cernansky NP, Curran HJ, Gaffuri P, Pitz WJ, Westbrook CK, A Flow Reactor Study of Neopentane Oxidation at 8 atmospheres: Experiments and modeling. Combust Flame 1999;118:415-30.

[69] Curran HJ, Gaffuri P, Pitz WJ, Westbrook CK, Leppard WR. Autoignition chemistry in a motored engine: An experimental and kinetic modeling study. Proc Combust Inst 1996;26:2669-77.

[70] Curran HJ, Gaffuri P, Pitz WJ, Westbrook CK. A Comprehensive study of n-heptane oxidation. Combust Flame 1998;114:149-77.

[71] Curran HJ, Gaffuri P, Pitz WJ, Westbrook CK. A Comprehensive study of iso-octane oxidation. Combust Flame 2002;129:253-80.

http://www-cmls.llnl.gov/?url=science and technology-chemistry-combustion

[72] Westbrook CK, Pitz WJ, Boercker JE, Curran HJ, Griffiths JF, Mohamed C et al. Detailed chemical kinetic reaction mechanisms for autoignition of isomers of heptane under rapid 
compression. Proc Combust Inst 2002;29:1311-18.

[73] Fisher EM, Pitz WJ, Curran HJ, Westbrook CK. Detailed chemical kinetic mechanisms for combustion of oxygenated fuels. Proc Combust Inst 2000;28:1579:86.

[74] Parsons B.I, Danby CJ. The oxidation of hydrocarbons and their derivatives. Part I. The observation of the progress of the reaction by pressure change and by analysis. J Chem Soc 1956:1795-98.

[75] Parsons B.I, Hinshelwood SC. The oxidation of hydrocarbons and their derivatives. Part II. Structural effects in the ester series. J Chem Soc 1956:1798-803S.

[76] Kojima S. Detailed modelling of n-butane autoignition chemistry. Combust Flame 1994;99-87-136.

[77] Ribaucour M, Minetti R, Sochet LR. Autoignition of n-pentane and 1-pentene: experimental data and kinetic modelling. Proc Combust Inst 2000;27:345-51.

[78] Böhm H, Baronnet F, El Kadi B. Chemical kinetic modeling of ETBE oxidation at low temperature. Oxidation Comm 1996;19:25-32.

[79] Böhm H, Baronnet F, El Kadi B. Comparative modeling study on the inhibiting effect of TAME, ETBE and MTBE at low temperature. Phys Chem Chem Phys 2000;2:1929-33.

[80] Gaïl S, Thomson MJ, Sarathy SM, Syed SA, Dagaut P, Diévart P, Marchese AJ, Dryer FL. A wide-range kinetic modeling study of methyl butanoate combustion. Proc Combust Inst 2007;31:305-11.

[81] Metcalfe WK, Dooley S, Curran HJ, Simmie JM, El-Nahas AM, Navarro MV. Experimental and modeling study of $\mathrm{C}_{5} \mathrm{H}_{10} \mathrm{O}_{2}$ ethyl and methyl esters. J Phys Chem 2007;11:4001-14.

[82] Tomlin AS, Turányi T, Pilling MJ. Mathematic tools for the construction, investigation and reduction of combustion mechanisms. In Comprehensive Chemical Kinetics: low- 
temperature combustion and autoignition, Pilling MJ Ed., 35, Elsevier, Amsterdam, 1997.

[83] Chinnick SJ. Computer based elucidation of reaction mechanisms.1987 Ph.D Thesis, University of Leeds.

[84] Chevalier C, Warnatz J, Melenk H. Hydrocarbon ignition: automatic generation of reaction mechanisms and application to modeling of engines knock. Bes Buns Phys Chem. 1990;94:1362-67.

[85] Chevalier C, Pitz WJ, Warnatz J, Westbrook CK, Melenk H. Hydrocarbon ignition: automatic generation of reaction mechanism and applications to modeling of engines knock. Proc Combust Inst 1992;24:93-101.

[86] Nehse M, Warnatz J, Chevalier C. Kinetic modeling of the oxidation of large aliphatic hydrocarbons. Proc Combust Inst 1996;26:773-80.

[87] Morley C. Automatic analysis of the mechanism for the auto-ignition of alkanes and ethers; correlation with octane number. Proceedings of the Anglo-German Combustion Symposium, Queen's College, Cambridge, 1993.

[88] Blurock ES. Reaction; system for modeling chemical reactions. J Chem Inf Comput Sci 1995;35:607-16.

[89] Moréac G, Blurock ES, Mauss F. Automatic generation of a detailed mechanism for the oxidation of n-decane. Combust Sci and Tech 2006;178:2025-38.

[90] Dente ME, Ranzi E. Pyrolysis, theory and industrial practice. In: Albright L, Bryce, Corcoran, eds. San Diego, Academic press, New York, 1983;7.

[91] Dente M, Bozzano G, Faravelli T, Marongiu A, Pierucci S, Ranzi E. Kinetic modeling of pyrolysis processes in gas and condensed phase. Adv Chem Eng 2007;32:51-166.

[92] Ranzi E, Dente M, Goldaniga A, Bozzano G, Faravelli T. Lumping procedures in detailed kinetic modeling of gasification, pyrolysis, partial oxidation and combustion of 
hydrocarbon mixtures. Prog Energy Combust Sci 2001;27:88-139.

[93] Ranzi E, Frassoldati A, Granata S, Faravelli T. Wide-range kinetic modeling study of the pyrolysis, partial oxidation and combustion of heavy n-alkanes. Ind Eng Chem Res $2005 ; 44: 5170-83$.

[94] Ranzi E, Faravelli T, Gaffuri P, Sogaro A. Low-temperature combustion: automatic generation of primary oxidation reactions and lumping procedures. Combust Flame 1995;102:179-92.

[95] Ranzi E, Sogaro A, Gaffuri P, Pennati G, Faravelli T. A wide-range modeling study of methane oxidation. Combust Sci and Tech 1994;96:279-325.

[96] Ranzi E, Gaffuri P, Faravelli T, Dagaut P. A wide-range modeling study of n-heptane oxidation. Combust Flame 1995;103:91-106.

[97] Ranzi E, Faravelli T, Gaffuri P, D’Anna A, Ciajolo A. A wide-range modeling study of Iso-octane oxidation. Combust Flame 1997;108:24-42.

[98] Goldaniga A, Faravelli T, Ranzi E, Dagaut P, Cathonnet M. The oxidation of oxygenated octane improvers : MTBE, ETBE, DIPE and TAME. Proc Combust Inst 1996;26:627-32.

[99] Haux L, Cunin PY, Griffiths M, Côme GM. Construction automatique d'un mécanisme de réaction radicalaire I - Principe. J Chim Phys 1985;82:1027-31.

[100] Haux L, Cunin PY, Griffiths M, Côme GM. Construction automatique d'un mécanisme de réaction radicalaire I - Algorithme général. J Chim Phys 1988;85:739-43.

[101] Warth V, Battin-Leclerc F, Fournet R, Glaude PA, Côme GM, Scacchi G. Computer based generation of reactions mechanisms for gas-phase oxidation. Comput. Chem 2000;24:54160.

[102] Barbé P, Battin-Leclerc F, Côme G.M. Experimental and modelling study of methane and ethane oxidation between 773 and 1573 K. J Chim Phys 1995;92:1666-92. 
[103] Warth V, Stef N, Glaude PA, Battin-Leclerc F, Scacchi G, Côme GM. Computed aided design of gas-phase oxidation mechanisms: Application to the modelling of normal-butane oxidation. Combust. Flame, 1998;114:81-102.

[104] Glaude PA, Battin-Leclerc F, Fournet R, Warth V, Côme GM, Scacchi G. Construction and simplification of a model of the oxidation of alkanes. Combust. Flame, 2000;122:45162.

[105] Glaude PA, Warth V, Fournet R, Battin-Leclerc F, Côme GM, Scacchi G. Modelling of n-heptane and iso-octane gas-phase oxidation at low temperature by using computer-aided designed mechanisms. Bull Soc Chim Belg 1997;106:343-48.

[106] Battin-Leclerc F, Fournet R, Glaude PA, Judenherc B, Warth V, Côme GM et al. Modeling of the gas-phase oxidation of decane from 600 to 1600 K. Proc Combust Inst 2000;28:1597-605.

[107] Glaude PA, Battin-Leclerc F, Judenherc B, Warth V, Fournet R, Côme GM et al. Experimental and modeling study of the gas-phase oxidation of methyl and ethyl tertiary butyl ethers. Combust Flame 2000;121:345-55.

[108] Carlier M, Fittschen C, Minetti R, Ribaucour M, Sochet LR. Experimental and modeling study of oxidation and autoignition of butane at high pressure. Combust Flame 1994;96:201-11.

[109] Chandraratna MR, Griffiths JF. Pressure and concentration dependences of the autoignition temperature for normal butane+air mixtures in closed vessel. Combust. Flame 1994;99: 291-306.

[110] Minetti R, Ribaucour M, Carlier M, Sochet LR. Autoignition delays of a series of linear and branched chain alkanes in the intermediate range of temperature. Combust Sci and Tech 1996;113-114:179-92. 
[111] Griffiths JF, Halford-Maw PA, Rose DJ. Fundamental features of hydrocarbon autoignition in a rapid compression machine. Combust Flame 1993;95:291-306.

[112] Griffiths JF, Halford-Maw PA, Mohamed C. Spontaneous ignition delays as a diagnostic of the propensity of alkanes to cause engine knock. Combust Flame 1997;111:327-37.

[113] Zhukov VP, Sechenov VA, Starikovkii AY. Self-ignition of a lean mixture of n-pentane and air over a wide range of pressures. Combust Flame 2005;140:196-203.

[114] Zhukov VP, Sechenov VA, Starikovkii AY. Ignition delay times in lean n-hexane/air mixture at high pressures. Combust Flame 2004;136:256-59.

[115] Silke EJ, Curran HJ, Simmie JM. The influence of fuel structure on combustion as demonstrated by the isomers of heptane: a rapid compression machine study. Proc Combust Inst 2004;30:2639-47.

[116] Minetti R, Carlier M, Ribaucour M, Therssen E, Sochet LR. A rapid compression machine investigation of oxidation and autoignition of n-heptane : Measurements and modeling. Combust. Flame 1995;102:298-309.

[117] Tanaka S, Ayala F, Keck JC, Heywood JB. Two-stage ignition in HCCI combustion and HCCI control by fuels and additives. Combust Flame 2003;132:219-39.

[118] Ciezki HK, Adomeit G. Shock-tube investigation of self-ignition of n-heptane-air mixtures under engine relevant conditions. Combust Flame1993;93:421-33.

[119] Fieweger K, Blumenthal R, Adomeit G. Self ignition of S.I. engines models fuels: a shock tube investigation at high pressure. Combust Flame 1997;109:599-619.

[120] Herzler J, Jerig L, Roth P. Shock tube study of the ignition of lean n-heptane/air mixtures at intermediate temperatures and high pressures. Proc Combust Inst. 2005;30:1147-53

[121] Gauthier BM, Davidson DF, Hanson RK. Shock tube determination of ignition delay times in full-blend and surrogate fuel mixtures. Combust Flame 2004;139:300-11. 
[122] Minetti R, Carlier M, Ribaucour M, Therssen E, Sochet LR. Comparison of oxidation and autoignition of the two primary reference fuels by rapid compression. Proc Combust Inst 1996;26:747-53.

[123] He X, Donovan MT, Zigler BT, Palmer TR, Walton SM, Wooldridge MS, Atreya A. An experimental and modeling study of iso-octane ignition delay times under homogeneous charge compression ignition conditions. Combust Flame 2005;142:266-75.

[124] He X, Zigler BT, Walton SM, Wooldridge MS, Atreya A. A rapid compression facility study of $\mathrm{OH}$ time histories during iso-octane igntion. Combust Flame 2006;145:552-70.

[125] Walton SM, He X, Zigler BT, Wooldridge MS, Atreya A. An experimental investigation of iso-octane ignition phenomena. Combust Flame 2007;150:246-62.

[126] Davidson DF, Gauthier GM, Hanson RK. Shock tube measurements of iso-octane/air and toluene/air at high pressure. Proc Combust Inst 2005;30:1175-82.

[127] Pfahl U, Fieweger K, Adomeit G. Shock tube investigation of ignition delay times of multicomponent fuel/air mixtures under engines relevant conditions. Subprogram FK4, IDEA-EFFECT, Final Report, 1996.

[128] Zhukov VP, Tsyganov DL, Sechenov VA, Starikovkii AY. N-decane ignition at high pressures. Proceedings of the $2^{\text {nd }}$ European Combustion Meeting, Louvain-la-Neuve, 2005.

[129] Kumar K, Mittal G, Sung CJ. Autoignition of n-decane under high pressure conditions. Proceedings of the $5^{\text {th }}$ US Combustion Institute Meeting, San Diego, 2007.

[130] Dagaut P, Cathonnet M. Oxidation of neopentane in a jet-stirred reactor from 1 to 10 atm: an experimental and detailed kinetic modelling study. Combust Flame 1999;118:191:203.

[131] Dagaut P, Reuillon M, Cathonnet M. High-pressure oxidation of liquid fuels from low to high-temperature. n-heptane and iso-octane. Combust Sci and Tech 1994;95:233-60.

[132] Dagaut P, Reuillon M, Cathonnet M. Experimental study of the oxidation of n-heptane in a 
jet-stirred reactor from low temperature to high temperature and pressures up to $40 \mathrm{~atm}$. Combust Flame 1995;101:132-40.

[133] Cavaliere A, Ciajolo A, D’Anna A, Mercoglioano R, Raguci R. Auto-ignition of n-heptane and n-tetradecane in engine-like conditions. Combust Flame 1993;93:279-86.

[134] Ciajolo A, D’Anna A, Mercoglioano R. Slow-combustion of n-heptane, iso-octane and a toluene/n-heptane mixture. Combust Sci and Tech 1993;90:357-71.

[135] Ciajolo A, D'Anna A. Controlling steps in the low-temperature oxidation of n-heptane and iso-octane. Combust Flame 1998;112:617-22.

[136] Callahan CV, Held TJ, Dryer FL, Minetti R, Ribaucour M, Sochet LR, et al. Experimental data and kinetic modelling of primary reference fuel mixtures. Proc Combust Inst 1996;26: 739-46.

[137] Dagaut P, Reuillon M, Cathonnet M. High pressure oxidation of liquid fuel from low to high temperature. 3. n-Decane. Combust Sci and Tech 1994;103:349-59.

[138] Natelson R, Johnson R, Kurman M, Cernansky N, Miller D. Low temperature oxidation of selected jet fuel and diesel fuel components at elevated pressure. Proceedings of the $5^{\text {th }}$ US Combustion Institute Meeting, San Diego, 2007.

[139] Kurman M, Natelson R, Cernansky N, Miller D. Intermediare species analysis of FischerTropsch JP-8 surrogate components in the low and intermediate temperature reaction regime. Proceedings of the $5^{\text {th }}$ US Combustion Institute Meeting, San Diego, 2007.

[140] Agosta A, Cernansky NP, Miller DL, Faravelli T, Ranzi E. Reference components of jet fuels: kinetic modeling and experimental results. Exp Therm Fluid Sci; 2004;28:701-8.

[141] Lenhert DB, Cernansky NP, Miller DL. Oxidation of large molecular weight hydrocarbons in a pressurized flow reactor. Proceedings of the $4^{\text {th }}$ Joint Meeting of the U.S. Sections, Philadelphia, 2005. 
[142] Donovan MT, He X, Zigler BT, Palmer TR, Wooldridge MS, Atreya A. Demonstration of a free-piston rapid compression facility for the study of high temperature combustion phenomena. Combust Flame 2004;137:351-65.

[143] Mittal G, Sung CJ. A rapid compression machine for chemical kinetics studies at elevated pressures and temperatures. Combust Sci and Tech 2007;179:487-530.

[144] Lee D, Hochgreb S. Rapid compression machines: Heat transfer and suppression of corner vortex. Combust. Flame 1998;114:531-45.

[145] Mittal G, Sung CJ. Aerodynamics inside a rapid compression machine. Combust. Flame 2006;145:160-80.

[146] Clarkson J, Griffiths J, MacNamara JP, Whitaker BJ. Temperature fields during the development of combustion in a rapid compression machine. Combust Flame 2001;125:1162-75.

[147] Cherneskey M, Bardwell J. Surface effects in butane oxidation. Canad J Chem 1960;38:482-92.

[148] Aggarwal SK. A review of spray ignition phenomena: present status and future research. Prog Energy Combust Sci 1998;24:565-600.

[149] Moriue O, Eigenbrod C, Rath HJ, Sato J, Okai K, Tsue M, et al. Effects of dilution by aromatic hydrocarbons on staged ignition behavior of n-decane droplets. Proc Combust Inst 2000;28:969-75.

[150] Xu G, Ikegami M, Honna S, Ikeda K, Ma X, Nagaishi H et al. Inverse influence of initial diameter on droplet burning rate in cold and hot ambiences: a thermal action of flame in balance with heat loss. Int J Heat Mass Transfer 2003;46:1155-69.

[151] Mittal G, Sung CJ, Fairweather M, Tomlin AS, Griffiths JF, Hughes KJ. Significance of thr $\mathrm{HO}_{2}+\mathrm{CO}$ reaction during the combustion of $\mathrm{CO}+\mathrm{H}_{2}$ mixtures at high pressures. Proc 
Combust Inst 2007;31:419-27.

[152] Matras D, Villermaux J. Un réacteur continu parfaitement agité par jets gazeux pour l'étude cinétique de réaction chimiques rapides. Chem Eng Sci 1973;28 :129-37.

[153] Buda F. Mécanismes cinétiques pour l'amélioration de la sécurité des procédés d'oxydation des hydrocarbures. 2006 Ph.D Thesis, INPL, Nancy. http://tel.archivesouvertes.fr/

[154] Battin-Leclerc F, Buda F, Fairweather M, Glaude PA, Giffiths JF, Hughes KJ, Porter R, Tomlin AS. A Numerical study of the kinetic origins of two-stage autoignition and the dependence of autoignition temperature on reactant pressure in lean alkane-air mixtures. Proceedings of the $2^{\text {nd }}$ European Combustion Meeting, Louvain-la-Neuve, 2005.

[155] Cuoci A, Mehl M, Buzzi-Ferraris G, Faravelli T, Manca D, Ranzi E. Autoignition and burning rates of fuel droplets under microgravity. Combust Flame 2005;143:211-26.

[156] Ciajolo A, D’anna A, Kurz M. Low-temperature oxidation of MTBE in a high pressure jetstirred flow reactor. Combust Sci and Tech 1997;123:49-61.

[157] Sarathy SM, Gaïl S, Syed SA, Thomson MJ, Dagaut P. A comparison of saturated and unsaturated $\mathrm{C}_{4}$ fatty acid methyl esters in an opposed flow diffusion flame and a jet stirred reactor. Proc Combust Inst 3007;31:1015-22.

[158] Schwatz WJ, McEnally CS, Pfefferle LD. Decomposition and hydrocarbon growth processes in non-premixed flames. J Phys Chem A 2006;110:6643-48.

[159] DeSain JD, Klippenstein SJ, Miller JA, Taatjes CA. Measurements, Theory, and Modeling of $\mathrm{OH}$ Formation in Ethyl $+\mathrm{O} 2$ and Propyl $+\mathrm{O} 2$ Reactions. J. Phys. Chem. A $2003 ; 107: 4415-27$

[160] Xi Z, Han W-J, Bayes KD. Temperature Dependence of the Rate Constant for the Reaction of Neopentyl Radicals with $\mathrm{O}_{2}$. J. Phys. Chem 1988;92:3450-53. 
[161] Robertson SH, Seakins PW, Pilling MJ. Elementary reactions. In Comprehensive Chemical Kinetics: low-temperature combustion and autoignition, Pilling MJ Ed., 35, Elsevier, Amsterdam, 1997.

[162] Glaude PA. Construction automatique et validation de modèles cinétiques de combustion d'alcanes et d'éthers. 1999 Ph.D Thesis, INPL, Nancy.

[163] Baldwin RR, Hisham MW, Walker RW. Arrhenius parameters of elementary reactions involved in the oxidation of neopentane. J Chem Soc Faraday Trans I 1982;78:1615-27.

[164] Hughes KJ, Halford-Maw PA, Lightfoot PD, Turányi T, Pilling MJ. Direct measurements of the neo-pentyl peroxy-hydroperoxy radical isomerization over the temperature range 660-750 K. Proc Combust Inst 1992;24:645-52.

[165] Chan W-T, Pritchard H.O., Hamilton I.P. Self abstraction in aliphatic hydroperoxyl radicals. J Chem Soc, Faraday Trans 1998;94:2303-6.

[166] Bozzelli JW, Ritter ER. Chemical and physical processes in combustion. The Combustion Institute, Pittsburg, 1993;103:459.

[167] Lightfoot PD, Cox RA, Crowley JN, Destriau M, Hayman GD, Jenkin ME et al. Organic peroxy radicals: kinetics, spectroscopy and tropospheric chemisty. Atmospheric Chemistry a-General Topics 1992;26:1805-961.

[168] Chan W-T, Pritchard H.O., Hamilton I.P. Dissociative ring-closure in aliphatic hydroperoxy radicals. Phys Chem Chem Phys 1999;1:3715-19.

[169] Chen C-J, Bozzellli JW. Kinetic analysis for $\mathrm{HO}_{2}$ addition to ethylene, propene, and isobutene, and thermochemical parameters of alkyl hydroperoxides and hydroperoxide alkyl radicals. J Phys Chem A 2000;104:4997-5012.

[170] Baldwin RR, Dean CE, Walker RW. Relative rate study of the addition of $\mathrm{HO}_{2}$ radicals to $\mathrm{C}_{2} \mathrm{H}_{4}$ and $\mathrm{C}_{3} \mathrm{H}_{6}$. Chem Soc Faraday Trans 2 1986;81:1445-55. 
[171] Allara LR, Shaw RA. compilation of kinetic parameters for the thermal degradation of nalkanes molecules. J Phys Chem Ref Data 1980;3:523-59.

[172] Sahetchian KA, Rigny R, Tardieu de Maleissye J, Batt L, Anwar Khan M, Mathews S. The pyrolysis of organic hydroperoxides (ROOH). Proc Combust Inst 1992;24:637-43.

[173] Daly NJ, Wentrup C. The thermal decomposition of t-butyl ethyl ether. Austral J Chem $1968 ; 21: 1535-39$.

[174] El-Nahas AM, Navarro MV, Simmie JM, Bozzelli JW, Curran HJ, Dooley S, Metcalfe W. Enthalpies of formation, bond dissociation energies and reaction paths for the decomposition of model biofuels: ethyl propanoate and methyl butanoate. J Phys Chem A 2007;111:3727:39.

[175] Truhlar DG, Garrett BC, Klippenstein SJ. Current state of transition-state theory. J Phys Chem 1996;100:12771-800.

[176] Cramer CJ. Essentials of computational chemistry - Theory and models. $2^{\text {nd }}$ ed., John Wiley \& Sons, Chichester, 2004.

[177] Harding LB, Klippenstein SJ, Jasper AW. Ab inition methods for reactive potential surfaces. Phys Chem Chem Phys 2007;9:4055-70.

[178] Fernándo-Ramos A, Miller JA, Klippenstein SJ. Modelling the kinetics of bimolecular reactions. Chem Rev 2006;106:4518-84.

[179] Miller JA, Klippenstein SJ. Master equation methods in gas phase chemicel kinetics. J Phys Chem A 2006;110:10528-44.

[180] Weber de Menezes E, Cataluna R, Samios D, Da Silva R. Addition of an azeotropic ETBE/ethanol mixture in eurosuper-type gasolines. Fuel 2006;2567-77.

[181] Balwin RR, Walker RW. Elementary reactions in the oxidation of alkenes. Proc Combust Inst 1980;18:819-29. 
[182] Balwin RR, Dean CE, Walker RW. Relative rate study of addition of $\mathrm{HO}_{2}$ radicals to $\mathrm{C}_{2} \mathrm{H}_{4}$ and $\mathrm{C}_{3} \mathrm{H}_{6}$. J Chem Soc Faraday Trans 1986;82:1445-57.

[183] Lodhi ZH, Walker RW. Oxidation of allyl radicals: Kinetic parameters for the reactions of allyl radicals with $\mathrm{HO}_{2}$ and $\mathrm{O}_{2}$ between 400 and $480^{\circ} \mathrm{C}$. J Chem Soc Faraday Trans 1991;87(15):2361-65.

[184] Stothard ND, Walker RW. Oxidation chemistry of propene in the autoignition region: Arrhenius parameters for the allyl $+\mathrm{O} 2$ reactions pathways and kinetic data for initiation reactions. J Chem Soc Faraday Trans 1992;88(18):2621-29.

[185] Ingham T, Walker RW, Woolford RE. Kinetic parameters for the initiation reaction RH + $\mathrm{O}_{2} \rightarrow \mathrm{R}+\mathrm{HO}_{2}$. Proc Combust Inst 1994;25:767-74.

[186] Touchard S, Fournet R, Glaude PA, Warth V, Battin-Leclerc F, Vanhove G, Ribaucour M, Minetti R. Modeling of the oxidation of large alkenes at low temperature. Proc Combust Inst 2005;30:1073-81.

[187] Knyazev VD, Slagle IR. Thermochemistry and kinetics of the reaction of 1-methylallyl radicals with molecular oxygen. J Phys Chem A 1998;102:8932-40.

[188] Leppard WR. The chemical origin of fuel octane sensitivity. 1990;SAE 902137.

[189] Stark MS. Epoxidation of alkenes by peroxyl radicals in the gas phase: structure-activity relationships. J Phys Chem A 1997;101:8296-301.

[190] Stark MS, Waddington RW. Oxidation of propene in the gas phase. Int J Chem. Kin $1995 ; 27: 123-51$.

[191] Wilk RD, Cernansky NP, Pitz WJ, Westbrook CK. Propene oxidation at low and intermediate temperatures: a detailed chemical kinetic study. Combust Flame 1989;77:14570.

[192] Heyberger B, Battin-Leclerc F, Warth V, Fournet R, Côme GM, Scacchi G. 
Comprehensive mechanism for the gas-phase oxidation of propene. Combust Flame 2001;126:1780-802.

[193] Wilk RD, Cernansky NP, Cohen RS. An experimental study of propene oxidation at low and intermediate temperatures. Combust Sci and Tech 1987;52:39-58.

[194] Pitz WJ, Westbrook CK, Leppard WR. Autoignition chemistry of $\mathrm{C}_{4}$ olefins under motored engine conditions: a comparison of experimental and modeling results. 1991;SAE paper 912315.

[195] Dagaut P, Cathonnet M. Isobutene oxidation and ignition: experimental and detailed kinetic modelling study. Combust Sci and Tech 1998;137:237-75.

[196] Baugé JC, Battin-Leclerc F, Baronnet F. Experimental and modelling study of oxidation of isobutene. Int J Chem Kinet 1998;30:629-40.

[197] Minetti R, Roubaud A, Therseen E, Ribaucour M, Sochet LR. The chemisty of pre-ignition of n-pentane and 1-pentene. Combust Flame. 1999;118:213-20.

[198] Prabhu SP, Bhat RK, Miller DL, Cernansky NP. 1-pentene oxidation and its interaction with nitric oxide in the low and negative temperature coefficient regions. Combust Flame, 1996;104:377-90.

[199] Vanhove G, Ribaucour M, Minetti R. On the influence of the position of the double bond on the low-temperature chemistry of hexenes. Proc Combust Inst 2005;30:1065-72.

[200] Yahyaoui M, Djebaïli-Chaumeix N, Dagaut P, Paillard C-E. Kinetics of 1-hexene oxidation in a JSR and a shock tube : Experimental and modeling study. Combust Flame 2006;147:67-78.

[201] Mehl M, Faravelli T, Ranzi E, Ciajolo A, D’Anna A, Tregrossi A. A wide range kinetic modeling study of alkene oxidation. Proceedings of the $29^{\text {th }}$ Combustion Meeting. Italian Section of the Combustion Institute (2006). 
[202] Mehl M, Faravelli T, Giavazzi F, Ranzi E, Scorletti P, Tardani A, Terna D. Detailed chemistry promotes understanding of octane numbers and gasoline sensitivity. Energy Fuels 2006;20:2391-98.

[203] Touchard S. Construction et validation de modèles cinétiques détaillés pour la combustion de mélanges modèles des essences. 2005 Ph.D Thesis, INPL, Nancy. http://tel.archivesouvertes.fr/

[204] Heyberger B. Mécanismes de combustion d'alcanes, d'alcènes et de cyclanes. 2002 Ph.D Thesis, INPL, INPL, Nancy.

[205] Heyberger B, Belmekki N, Conraud V, Glaude PA, Fournet R, Battin-Leclerc F. Oxidation of small alkenes at high temperature. Int J Chem Kin 2002;34:666-77.

[206] Battin-Leclerc F. Development of kinetic models for the formation and degradation of unsaturated hydrocarbons at high temperature. Phys Chem Chem Phys 2002;2:2072:78.

[207] Yahyaoui M, Djebaïli-Chaumeix N, Paillard C-E, Touchard S, Fournet R, Glaude PA, Warth V, Battin-Leclerc. Experimental and modelling study of 1-hexene oxidation behind reflected shock waves. Proc Combust Inst 2005;30:1137-45.

[208] Touchard S, Buda F, Dayma G, Glaude PA, Fournet R, Battin-Leclerc F. Experimental and modeling study of the oxidation of 1-pentene at high temperature. Int $\mathrm{J}$ Chem Kin 2005;37:451-63.

[209] Tsang W. Heat of Formation of Organic Radicals by Kinetic Methods. In Energetics of organic free radicals. Eds. Simões JAM, Greenberg A, Liebman JF. 4 Ed. Kluwer Academic Publishers, Dordrecht, 1996.

[210] Luo YR. Handbook of bond dissociation energies in organic compounds. CRC Press, Boca Raton 2003.

[211] Denisov ET, Denisova TG. Handbook of antioxidants. CRC Press, New York 2000. 
[212] Hoyermann K, Sievert R. Die reaktion von OH-radikalen mit propen: I. bestimmung der primärprodukte bei niedrigen druecken. Ber Bunsenges Phys Chem 1979;83:933-39.

[213] Morgan CA, Pilling MJ, Tulloch JM, Ruiz RP, Bayes KD. Direct determination of the equimilbrium constant and thermodynamic parameters for the reaction $\mathrm{C}_{3} \mathrm{H}_{5}+\mathrm{O}_{2} \leftrightarrow \mathrm{C}_{3} \mathrm{H}_{5} \mathrm{O}_{2}$. J Chem Soc Faraday Trans 1982;78(2):1323-1330.

[214] Perrin D, Richard C, Martin R. H2S-promoted thermal isomerization of cis-2-pentene to 1-pentene and trans-2-pentene around 800 K. Int J Chem Kin 1988;20: 621-632.

[215] Miyoshi A, Masui H, Washida N. Rates of Reaction of Hydroxyalkyl Radicals with Molecular Oxygen. J Phys Chem 1990;94:3016-3019.

[216] Gierczak T, Gawlowski J, Niedzielski J. Mutual isomerization of cyclopentyl and 1-Penten-5-y1 radicals. Int J Chem Kin 1986;18:623-637.

[217] Pedley JB, Naylor RD, Kirby SP. Thermochemical data of organic compounds. $2^{\text {nd }}$ ed., Chapman and Hall. London, 1986.

[218] Söderbergh B, Robelius F, Aleklett K. A crash programme scenario for the Canadian oil sands industry. Energy policy 2007;35:1931-47.

[219] Wilson MF, Fisher IP, Kriz JF. Hydrogenation and extraction of aromatics from oil sans distillates and effects on cetane improvements. Energy Fuels 1987;1:540-44.

[220] Gulati SK, Walker RW. Addition of cyclohexane to slowly reacting $\mathrm{H}_{2}-\mathrm{O}_{2}$ mixtures at $480^{\circ} \mathrm{C}$. J Chem Soc Faraday Trans 2 1989;85(11):1799-812.

[221] Handford-Styring S, Walker RW. Addition of cyclopentane to slowly reacting mixtures of $\mathrm{H}_{2}+\mathrm{O}_{2}$ between 673 and $783 \mathrm{~K}$ : reactions of $\mathrm{H}$ and $\mathrm{OH}$ with cyclopentane and of cyclopentyl radicals. J Chem Soc Faraday Trans 2 1995;91:1431-38.

[222] Klaï SE, Baronnet F. Etude de l'oxydation homogène du cyclohexane en phase gazeuse. I. Etude expérimentale. J Chim Phys 1993;90:1929-50. 
[223] Klaï SE, Baronnet F. Etude de l'oxydation homogène du cyclohexane en phase gazeuse. II. Mécanisme réactionnel et modélisation. J Chim Phys 1993;90:1951-98.

[224] Silke EJ, Pitz WJ, Westbrook CK, Ribaucour M. Detailed chemical kinetic modeling of cyclohexane oxidation. J Phys Chem A 2007;111:3761:75.

[225] Pitz WJ, Naik CV, Ni Mhaoldùin T, Westbrook CK, Curran HJ, Orme JP, Simmie JM. Modeling and experimental investigation of methylcyclohexane ignition in a rapid compression machine. Proc Combust Inst 2007;31:267-75.

[226] Granata S, Faravelli T, Ranzi E. A wide range kinetic modeling study of the pyrolysis and combustion of naphthenes. Combust Flame 2003;132:533-44.

[227] Cavalotti C, Rota R, Faravelli T, Ranzi E. Ab initio evaluation of primary cyclo-hexane oxidation reactions rates. Combust Flame 2007;31:201-9.

[228] Buda F, Heyberger B, Fournet R, Glaude P-A, Warth V, Battin-Leclerc F. Modeling of the gas-phase oxidation of cyclohexane. Energy Fuels 2006;20:1450-59.

[229] Lemaire O, Ribaucour M, Carlier M, Minetti R. The Production of Benzene in the LowTemperature Oxidation of Cyclohexane, Cyclohexene, and Cyclohexa-1,3-diene. Combust Flame 2001;127:1971-80.

[230] Voisin D, Marchal A, Reuillon M, Boettner JC, Cathonnet M. Experimental and kinetic modelling study of cyclohexane oxidation in a JSR at high pressure. Combust Sci Technol 1998;138:137-58.

[231] Dagaut P. On the kinetics of hydrocarbons oxidation from natural gas to kerosene and diesel fuel. Phys Chem Chem Phys 2002;4:2079-94.

[232] Vasu SS, Parikh NN, Davidson DF, Hanson RK. Methylcyclohexane oxidation: Shock tube experiments and modeling over a wide range of pressures and temperatures. Proceedings of the $5^{\text {th }}$ US Combustion Institute Meeting, San Diego, 2007. 
[233] Zeppieri Z, Brezinsky K, Glasman I. Pyrolysis studies of methylcyclohexane and oxidation Studies of methylcyclohexane and methylcyclohexane/toluene blends. Combust Flame; 1997;108:266-86.

[234] Davidson DF, Horning DC, Herbon JT, Hanson RK. Shock Tube Measurements of JP-10 Ignition. Proc Combust Inst 2000;28:1687-92.

[235] Handford-Styring SM, Walker RW. Arrhenius parameters for the $\mathrm{HO} 2+$ cyclohexane between 673 and $773 \mathrm{~K}$, and for $\mathrm{H}$ atom in cyclohexylperoxy radicals. Phys Chem Chem Phys 2001;3:2043-52.

[236] Carstensen H, Naik CV, Dean AM. Detailed modelling of the reaction of C2H5+O2. J Phys Chem A 2005;109:2264-81.

[237] Brezinsky K. The high temperature oxidation of aromatic hydrocarbons. Prog Energ Combust Sci 1986;12:1-24.

[238] Nicovich JM, Gump CA, Ravishankara AR. Rates of reactions of $\mathrm{O}\left({ }^{3} \mathrm{P}\right)$ with benzene and toluene. J Phys Chem 1982;86:1684-90.

[239] Colussi AJ, Zabel F, Benson SW. The very low-pressure pyrolysis of phenyl ethyl ether, phenyl allyl ether, and benzyl methyl ether and the enthalpy of formation of the phenoxy radical. Int J Chem Kin 1977;9:161-78.

[240] Lovell AB, Brezinsky K, Glassman I. Benzene oxidation perturbed by $\mathrm{NO}_{2}$ addition. Proc Combust Inst 1988;22:1063-74.

[241] Nicovich JM, Ravishankara AR. Reactions of hydrogen atom with benzene and toluene: kinetics and mechanism. J Phys Chem 1984;88:2534:51.

[242] Brezinsky K, Lintzinger T, Glassman I. The high temperature oxidation of the methyl side chain toluene. Int J Chem Kin 1984;16:1053-74.

[243] Ellis C, Scott M, Walker RW. Addition of toluene and ethylbenzene to mixture of $\mathrm{H}_{2}$ and 
$\mathrm{O}_{2}$ at $773 \mathrm{~K}$. Combust Flame 2003;132:291-304.

[244] Roubaud A, Minetti R, Sochet LR. High pressure auto-ignition and oxidation mechanisms of o-xylene, o-ethyltoluene, and n-butylbenzene between 600 and $900 \mathrm{~K}$. Combust Flame 2000;123:561-71.

[245] Shaddix CR, Brezinsky K, Glassman I. Analysis of fuel decay routes in the hightemperature oxidation of 1-methylnaphthalene. Combust Flame 1997;108:139-57.

[246] Bittker DA. Detailed mechanism for oxidation of benzene. Combust Sci and Tech 1991;79:49-72.

[247] Emdee JL, Brezinsky K, Glassman I. A Kinetic Model for the Oxidation of Toluene near 1200 K. J Phys Chem 1992;96:2151-61.

[248] Lindstedt RP, Skevis G. Detailed kinetic modelling of premixed benzene flame. Combust Flame 1994;99:551-61.

[249] Zhang HY, Mc Kinnon JT. Elementary reaction modelling of high-temperature benzene combustion. Combust Sci and Tech 1995;107:261-300.

[250] Tan Y, Frank P. A detailed comprehensive kinetic model for benzene oxidation using the recent kinetic results. Proc Combust Inst 1996;26:677-84.

[251] Shandross RA, Logwell JP, Howard JB.Destruction of benzene in high-temperature flames: chemistry of benzene and phenol. Proc Combust Inst 1996;26:711-19.

[252] Richter H, Benish TG, Mazyar OA, Green WH, Howard JB. Formation of polycyclic aromatic hydrocarbons and their radicals in a nearly sooting premixed benzene flame. Proc Combust Inst 2000;28:2609-18.

[253] Bittner JD, Howard JB. Composition profiles and reaction mechanisms in a near-sooting premixed benzene/oxygen/argon flame. Proc Combust Inst 1983;18:1105-16.

[254] Alzueta MU, Glarborg P, Dam-Johansen K. Experimental and kinetic modeling study of 
the oxidation of benzene. Int J Chem Kin 2000;32:498-522.

[255] Ristori A, Dagaut P, El Bakali A, Pengloan G, Cathonnet M. Benzene oxidation: Experimental results in a JSR and comprehensive kinetic modeling in JSR, shock-tube and flame. Combust Sci and Tech 2001;167:223-56.

[256] Da Costa I, Fournet R, Billaud F, Battin-Leclerc F. Experimental and modeling study of the oxidation of benzene. Int J Chem Kin 2003;35:503-24.

[257] Schöbel-Ostertag A, Braun-Unkhoff M, Wahl C, Krebs L. The oxidation of benzene under conditions encountered in waste incinerators. Combust Flame 2005;140:359-70.

[258] Lindstedt RP, Maurice LQ. Detailed kinetic modelling of toluene combustion. Combust Sci and Tech 1996;120:119-67.

[259] Klotz SD, Brezinsky K, Glassman I. Modeling the combustion of toluene-butane blends. Proc Combust Inst 1998;27:337-44.

[260] Sivaramakrishnan R, Tranter RS, Brezinsky K. High-pressure, high-temperature oxidation of toluene. Combust Flame 2004;139:340-50.

[261] Andrae JCG, Björnbom P, Cracknell RF, Kalghatgi GT. Autoignition of toluene reference fuels at high pressures modeled with detailed chemical kinetics. Combust Flame. 2007;149:2-24.

[262] Chaos M, Zhao Z, Kazakov A, Gokulakrisnan P, Angioletti M, Dryer FL. A PRF+toluene surrogate fuel model for simulating gasoline kinetics. Proceedings of the $5^{\text {th }}$ US Combustion Institute Meeting, San Diego, 2007.

[263] Djurisic ZM, Joshi AV, Wang H. Detailled kinetic modelling of benzene and toluene combustion. Proceedings of the $2^{\text {nd }}$ Joint Meeting of the US Sections of the Combustion Institute, Oakland, 2001.

[264] Pitz WJ, Seiser R, Bozzelli JW, Da Costa I, Fournet R, Billaud F, Battin-Leclerc F, 
Seshadri K, Westbrook CK. Chemical characterisation of combustion of toluene. Proceedings of the $2^{\text {nd }}$ Joint Meeting of the US Sections of the Combustion Institute, Oakland, 2001.

[265] Dagaut P, Pengloan G, Ristori A. Oxidation, ignition and combustion of toluene: experimental and detailed chemical kinetic modelling. Phys Chem Chem Phys 2002;4:1846-54.

[266] Bounaceur R, Da Costa I, Fournet R, Billaud F, Battin-Leclerc F. Experimental and modeling study of the oxidation of toluene. Int J Chem Kin 2005;37:25-49.

[267] Gaïl S, Dagaut P. Experimental kinetic study of the oxidation of p-xylene in a JSR and comprehensive detailed chemical kinetic modelling. Combust Flame 2005;141:281-97.

[268] Gaïl S, Dagaut P. Oxidation of m-xylene in a JSR Experimental study and detailed chemical kinetic modelling. Combust Sci and Tech 2007;179:813-44.

[269] Dagaut P, Ristori A, El Bakali A, Cathonnet M. Experimental and modeling study of the oxidation of n-propyl benzene. Fuel 2002;81:173-84.

[270] Battin-Leclerc F, Bounaceur R, Belmekki N, Glaude PA. Experimental and modeling study of the oxidation of xylenes. Int J Chem Kin 2006;38:284-302.

[271] Emdee JL, Brezinsky K, Glassman I. High-Temperature Oxidation Mechanisms of m- and p -Xylene. J Phys Chem 1991;95:1626-35.

[272] Emdee JL, Brezinsky K, Glassman I. Oxidation of o-xylene. Proc Combust Inst $1990 ; 23: 77-84$

[273] Ribaucour M, Roubaud A, Minetti R, Sochet LR. The low-temperature autoignition of alkylaromatics: esperimental study and modelling of the oxidation of n-butylbenzene. Proc Combust Inst 2000;28:1701-7.

[274] Pitsch H. Detailed kinetic reaction mechanism for ignition and oxidation of 
a-methylnaphthalene. Proc Combust Inst 1996;26:721-28.

[275] Mittal G, Sung C-J. Autoignition of toluene and benzene at elevated pressures in a rapid compression machine. Combust Flame 2007;150:355-68.

[276] Chai Y, Pfefferle LD. An experimental study of benzene oxidation at fuel-lean and stoichiometric equivalence ratio conditions. Fuel 1998;77:313-20.

[277] Roubaud A, Minetti R, Sochet LR. Oxidation and combustion of low alkyl benzenes at high pressure: comparative reactivity and auto-ignition. Combust Flame 2000;121:535-41.

[278] Marchal A. Etude de la contribution des familles chimiques constitutives des gazoles à la formation de polluants non réglementés. 1997 Ph.D Thesis, Université d'Orléans.

[279] Gail S, Dagaut P. Meta and para-xylene Oxidation: Experimental results in a JSR, comprehensive kinetic modeling. Proceedings of the $2^{\text {nd }}$ European Combustion Meeting, Louvain-la-Neuve, 2005.

[280] Litzinger TA, Brezinsky K, Glassman I. The oxidation of ethyl benzene near $1060 \mathrm{~K}$. Combust Flame 1986;63:251-67.

[281] Litzinger TA, Brezinsky K, Glassman I. Reactions of n-propyl benzene during gas phase oxidation. Combust Sci and Tech 1986;50:117-33.

[282] Crochet M, Vanhove G, Minetti R, Ribaucour M. An experimental and modelling study of the low-temperature autoignition of n-butylbenzene in lean conditions. Proceedings of the $3^{\text {rd }}$ European Combustion Meeting, Chania, 2007.

[283] Brezinsky K, Linteris G, Litzinger TA, Glassman I. High temperature oxidation of n-alkylbenzenes. Proc Combust Inst 1986;21:833-40.

[284] Shaddix CR, Brezinsky K, Glassman I., Oxydation of 1-methylnaphtalene. Proc Combust Inst;24,683:90.

[285] Dagaut P, Ristori A, Pengloan G, Cathonnet M. Kinetic effect of dimethoxymethane on the 
oxidation of indane. Energy Fuel. 2001;15:372-76.

[286] Bounaceur R, Battin-Leclerc F. Previously unpublished data (2004).

[287] Alzueta MU, Oliva M, Glarborg P. Parabenzoquinone pyrolysis and oxidation in a flow reactor. Int J Chem Kin 1998;30:683-97.

[288] Buth R., Hoyermann K., Seeba J. Reaction of phenoxy radicals in the gas phase. Proc Combust Inst 1994;25:841-49.

[289] Skokov S, Kazakov A, Dryer FL. A theoretical study of oxidation of phenoxy and benzyl radicals by $\mathrm{HO}_{2}$. Proceedings of the $4^{\text {th }}$ US Combustion Institute Meeting, Philadelphia, 2007.

[290] Volamer R, Klotz B, Barnes I, Imamura T, Wirtz K, Washida N, Becker KH, Platt U. $\mathrm{OH}$-initiated oxidation of benzene. Part II. Phenol formation under atmospheric conditions. Phys Chem Chem Phys 2002;4:1598-610.

[291] Frank P, Herzler J, Just Th, Wahl C. High-temperature reactions of phenyl oxidation. Proc Combust Inst 1994;25:833-40.

[292] $\mathrm{Yu}$ T, Lin MC. Kinetics of the $\mathrm{C}_{6} \mathrm{H}_{5}+\mathrm{O}_{2}$ reaction at low temeratures. J Am Chem Soc $1994 ; 116: 9571-76$

[293] Bozzelli J, Sebbar N, Pitz W, Bochhorn H. Reaction of phenyl radical with $\mathrm{O}_{2}$ : Thermodynamic properties, important reaction paths and kinetics. Proceedings of the $2^{\text {nd }}$ Joint Meeting of the US Sections of the Combustion Institute, Oakland, 2001.

[294] Sebbar N, Bozzelli J, Bochhorn H. The phenyl $+\mathrm{O}_{2}$ reaction: Thermodynamics and kinetics. Proceedings of the $3^{\text {rd }}$ European Combustion Meeting, Chania, 2007.

[295] Sirjean B, Ruiz-Lopez MF, Glaude PA, Battin-Leclerc F, Fournet R. Theorical study of the thermal decomposition mechanism of phenylperoxy radical. Proceedings of the $2^{\text {nd }}$ European Combustion Meeting, Louvain-la-Neuve, 2005. 
[296] Tokmakov IV, KimG-S, Kislov VV, Mebel AM, Lin MC. The reaction of phenyl radical with molecular oxygen: a G2M study of the potential energy surface. J Phys Chem 2005;109:6114-27.

[297] Frisch MJ, Trucks GW, Schlegel HB, Scuseria GE, Robb MA, Cheeseman JR, et al. Gaussian03, revision B05; Gaussian, Inc.: Wallingford, CT, 2004.

[298] Davis WM, Heck SM, Pritchard HO. Theoretical study of benzyl radical reactivity in combustion systems. J Chem Soc, Faraday Trans 1998;94:2725-28.

[299] Hippler H, Reihs C, Troe J. Shock tube UV absorption of the oxidation of benzyl radicals. Proc Combust Inst 1990;23:37-43.

[300] Müller-Markgraf W, Troe J. Thermal decomposition of benzyl iodide and benzyl radicals in shock waves. J Phys Chem 1988;92:4899-905.

[301] Herzler J, Fikri M, Hitzbleck K, Starke R, Schulz C, Roth P, et al. Shock-tube study of the autoignition of n-heptane/toluene/air mixtures at intermediate temperatures and high pressures. Combust Flame 2007;149:25-31.

[302] Clothier PQE, Shen D, Pritchard HO. Stimulation of diesel-fuel ignition by benzyl radicals. Combust Flame. 1995;101:383-86.

[303] Fenter FF, Nozière B, Caralp F, Lesclaux R. Study of the kinetics and equilibrium of the benzyl-radical association reaction with molecular oxygen. Int $\mathrm{J}$ Chem Kin 1994;26:171:89.

[304] Zhong X, Bozzelli JW. Thermochemical and kinetic analysis of the $\mathrm{H}, \mathrm{OH}, \mathrm{HO}_{2}$, $\mathrm{O}$, and $\mathrm{O}_{2}$ association reactions with cyclopentadienyl radical. J Phys Chem A 1998;102:3537-55.

[305] Lifshitz A, Tamburu C, Suslensky A, Dubnikova F. Decomposition and ring expansion in methylcyclopentadiene: single-pulse shock tube and modelling study. Proc Combust Inst 2005;30:1039-47. 
[306] Melius CF, Colvin ME, Marinov NM, Pitz WJ, Senkan SM. Reaction mechanisms in aromatic hydrocarbon formation involving the $\mathrm{C}_{5} \mathrm{H}_{5}$ cyclopentadienyl moiety. Proc Combust Inst 1996;26:685-92.

[307] Marinov NM, Pitz WJ, Westbrook CK, Vincitore AL, Castaldi MJ, Senkan SM, et al. Aromatic and polycyclic aromatic hydrocarbon formation in a laminar premixed n-butane flame. Combust Flame 1998;114:192-213.

[308] Filley J, McKinnon JT. Dimerization of cyclopentadienyl radical to produce naphthalene. Combust Flame 2001;124:721-23.

[309] Violi A, Yan S, Edding EG, Sarofim AF, Granata S, Faravelli T, Ranzi E. Experimental formulation and kinetic model for JP-8 surrogate mixtures. Combust Sci and Tech 2002;174:399-417.

[310] Ranzi E. A wide-range modelling study of oxidation and combustion of transportation fuels and surrogate mixtures. Energy Fuels 2006;20:1024-32. http://www.chem.polimi.it/CRECKModeling/

[311] Pitz WJ, Cernansky NP, Dryer FL, Egolfopoulos FN, Farrell JT, Friend DG, Pitsch H. Development of an experimental database and chemical kinetic models for surrogate gasoline fuels. 2007;SAE 2007-01-0175.

[312] Farrell JT, Cernansky NP, Dryer FL, Friend DG, Hergart CA, Law CK, McDavid RM, Mueller CJ, Patel AK, Pitsch H. Development of an experimental database and chemical kinetic models for surrogate diesel fuels. 2007;SAE 2007-01-0201.

[313] Westbrook CK, Warnatz J, Pitz WJ. A detailed chemical kinetic reaction mechanism for the oxidation of iso-octane and n-heptane over an extended temperature range and its application to analysis of engines knock. Proc Combust Inst 1988;22:893-901.

[314] Vanhove G, Petit G, Minetti R. Experimental study of the kinetic interactions in the low- 
temperature autoignition of hydrocarbon binary mixtures and a surrogate fuel. Combust Flame 2006;145:521-32.

[315] Curran HJ, Pitz WJ, Westbrook CK, Callahan CV, Dryer FL. Oxidation of automotive primary reference fuels at elevated pressures. Proc Combust Inst 1998;27:379-87.

[316] Naik CV, Pitz WJ, Sjöberg M, Dec JE, Orme J, Curran HJ, et al. Detailed chemical kinetic modelling of a surrogate fuels for gasoline and application to an HCCI engine. Proceedings of the $4^{\text {th }}$ Joint Meeting of the US Sections of the Combustion Institute, Philadelphia, 2005.

[317] Naik CV, Pitz WJ, Westbrook CK, Sjöberg M, Dec JE, Orme J, et al. Detailed chemical kinetic modelling of a surrogate fuels for gasoline and application to an HCCI engine. 2005;SAE 2005-01-3741. Mechanism provided by the authors.

[318] Andrae J, Johansson D, Bjönbom P, Risberg P, Kalghatgi G. Co-oxidation in the autoignition of primary reference fuels and n-heptane/toluene blends. Combust Flame. 2005, $140: 267-86$.

[319] Vanhove G, El Bakali A, Ribaucour M, Minetti R. Detailed thermokinetic modelling of the low-temperature autoignition of a tertiary surrogate petrol fuel. Proceedings of the $3^{\text {rd }}$ European Combustion Meeting, Chania, 2007.

[320] Dagaut P, Reuillon M, Cathonnet M. High pressure oxidation of liquid fuel from low to high temperature. 2. Mixtures of n-heptane and iso-octane. Combust Sci and Tech 1994;103:315-36.

[321] Dagaut P, Koch R, Cathonnet M. the oxidation of n-heptane in the presence of oxygenated octane improvers: MTBE and ETBE. Combust Sci and Tech 1997;122:345-61.

[322] Douté C, Deflau JL, Akrich R, Vovelle C. Chemical structure of atmospheric pressure premixed n-decane and kerosene flame. Combust Sci and Tech 1995;106:327.

[323] Glaude PA, Conraud V, Fournet R, Battin-Leclerc F, Côme GM, Scacchi G et al. Modeling 
the oxidation of mixtures of primary reference automobile fuels. Energy Fuels 2002;16:1186-95.

[324] Pires da Cruz A, Pera C, Anderlohr J, Bounaceur R, Battin-Leclerc F. A complex chemical kinetic mechanism for the oxidation of gasoline surrogate fuels: n-heptane, iso-octane and toluene - Mechanism development and validation. Proceedings of the $3^{\text {rd }}$ European Combustion Meeting, Chania, 2007.

[325] Vanhove G, Minetti R, Touchard S, Fournet R, Glaude PA, Battin-Leclerc F. Experimental and modelling study of the autoignition of 1-hexene/iso-octane mixtures at low temperatures. Combust Flame 2006;145:272-81.

[326] Hartmann M, Fikri M, Starke R, Schulz C. Shock-tube investigation of ignition delay times of model fuels. Proceedings of the $3^{\text {rd }}$ European Combustion Meeting, Chania, 2007.

[327] Yahyaoui M. Etude cinétique de la formation de polluants à partir de mélanges representatifs des essences. 2005 Ph.D Thesis, Université d'Orléans.

[328] Mittal G, Sung CJ. Homogeneous charge compression ignition of binary blends relevant to gasoline surrogates. Proceedings of the $3^{\text {rd }}$ European Combustion Meeting, Chania, 2007.

[329] Yahyaoui M, Djebaïli-Chaumeix N, Dagaut P, Paillard C-E, Gail S. Experimental and modelling study of gasoline surrogate mixtures oxidation in jet stirred reactor and shock tube. Proc Combust Inst 2007;31:385-91.

[330] Fikri M, Herzler J, Starke R, Kalghatgi GT, Roth P, Schulz C. Autoignition of gasoline surrogates mixtures at intermediate temperatures and high pressures. Combust Flame 2007; in press.

[331] Frenklach M. Transforming data into knowledge - Process Informatics for combustion chemistry. Proc Combust Inst 2007;31:125-140.

[332] Law CK. Combustion at a crossroad: status and prospects. Proc Combust Inst 2007;31:1- 
29.

[333] Vajda S, Turányi T. Principal component analysis for reducing the Edelson-Field-Noyes model of the Belousov-Zhabotinsky reaction. J Phys Chem 1986;90:1664-70.

[334] Lu T, Law CK. Linear time reduction of large kinetic mechanisms with directed relation graph: n-heptane and iso-octane. Combust Flame 2006;144:24-36.

[335] Saylam A, Ribaucour M, Pitz WJ, Minetti R. Reduction of large detailed chemical kinetic mechanisms for autoignition using joint analyses od reaction rate and sensitivities. Int $\mathbf{J}$ Chem Kin 2007;39:181-96.

[336] Porter R, Fairweather M, Griffiths JF, Hughes KJ, Tomlin AS. Simulated autoignition temperature and oxidation of cyclohexane using a QSSA reduced mechanism. Proceedings of the $3^{\text {rd }}$ European Combustion Meeting, Chania, 2007.

[337] Fournet R, Warth V, Glaude PA, Battin-Leclerc F, Scacchi G, Côme GM. Automatic reduction of detailed mechanisms of combustion of alkanes by chemical lumping. Int $\mathrm{J}$ Chem Kin 2000;32:36-51.

[338] Brad RB, Tomlin AS, Fairweather M, Griffiths JF. The application of chemical reduction methods to a combustion system exhibiting complex dynamics. Proc Combust Inst 2007;31:455-63.

[339] Lam SH, Goussis DA. The CSP method for simplifying kinetics. Int J Chem Kin 1994;26:461-86.

[340] Mass U, Pope SP. Simplifying chemical kinetics: Intrinsic low-dimensional manifolds in composition space. Combust Flame 1992;88:239-64.

[341] Soyhan HS, Mauss F, Sorusbay C. Chemical kinetic modelling of combustion in internal combustion engines using reduced chemistry. Combust Sci and Tech 2002;174(11\&12):73-91. 
[342] Valorani M, Creta F, Donato F, Najm HN, Goussis DA. Skeletal mechanism generation and analysis for n-heptane with CSP. Proc Combust Inst 2007;21:483-90.

[343] Turányi T. Application of repromodeling for the reduction of combustion mechanisms. Proc Combust Inst 1994;25:948-56.

[344] Colin O, Pires da Cruz A, Jay S. Detailed chemistry-based auto-ignition model including low temperature phenomena applied to 3D engine calculations. Proc Combust Inst 2005;30:2649-56.

[345] Colin O, Pera C, Jay S. Detailed chemistry tabulation based on a FPI approach adapted and applied to 3-D internal combustion engine calculation. Proceedings of the $3^{\text {rd }}$ European Combustion Meeting, Chania, 2007. 
Table 1

Composition of a European commercial gasoline (Guibet, 1997 [11]).

Families of components (\%)

\begin{tabular}{|c|c|c|c|c|c|c|c|}
\hline \multirow{2}{*}{$\begin{array}{c}\text { Number of } \\
\text { atoms of } \\
\text { carbon }\end{array}$} & \multicolumn{3}{|c|}{ Saturated compounds } & \multicolumn{3}{|c|}{ Unsaturated compounds } & \multirow[b]{2}{*}{ Total } \\
\hline & $\begin{array}{l}\text { Linear } \\
\text { alkanes }\end{array}$ & $\begin{array}{c}\text { Branched } \\
\text { alkanes }\end{array}$ & Ethers & $\begin{array}{l}\text { Cyclic } \\
\text { alkanes }\end{array}$ & Alkenes & $\begin{array}{c}\text { Aromatic } \\
\text { compounds }\end{array}$ & \\
\hline 4 & 5.14 & 0.30 & & & 1.49 & & 6.93 \\
\hline 5 & 1.26 & 7.84 & 0.50 & & 10.11 & 0.50 & 19.71 \\
\hline 6 & 0.64 & 6.34 & 3.00 & 1.19 & 5.07 & 1.23 & 17.47 \\
\hline 7 & 0.65 & 3.22 & & 1.05 & 1.56 & 8.11 & 14.59 \\
\hline 8 & 0.48 & 11.47 & & 0.43 & 0.34 & 13.61 & 26.33 \\
\hline 9 & 0.11 & 1.12 & & 0.16 & 0.07 & 9.49 & 10.95 \\
\hline 10 & 0.01 & 0.09 & & 0.09 & 0.02 & 2.80 & 3.01 \\
\hline 11 & & 0.10 & & & & 0.25 & 0.35 \\
\hline 12 & & 0.61 & & & & & 0.61 \\
\hline 13 & & 0.01 & & & & & 0.01 \\
\hline Total & 8.29 & 31.10 & 3.50 & 2.92 & 18.66 & 35.49 & 99.96 \\
\hline
\end{tabular}




\section{Table 2}

Comparison between the size of the low temperature and high temperature mechanisms for alkanes, as generated by EXGAS-ALKANES software [24] and octane numbers (RON and MON) of several alkanes [11].

\begin{tabular}{|c|c|c|c|c|c|c|}
\hline \multirow{2}{*}{ Type of alkane } & \multicolumn{2}{|c|}{ Low temperature } & \multicolumn{2}{|c|}{ High temperature } & \multirow{2}{*}{ RON } & \multirow[b]{2}{*}{ MON } \\
\hline & $\begin{array}{l}\text { Number } \\
\text { of species }\end{array}$ & $\begin{array}{l}\text { Number } \\
\text { of } \\
\text { reactions }^{\mathrm{a}}\end{array}$ & $\begin{array}{l}\text { Number } \\
\text { of species }\end{array}$ & $\begin{array}{l}\text { Number } \\
\text { of } \\
\text { reactions }\end{array}$ & & \\
\hline n-butane & 128 & 731 & 80 & 585 & 95 & 92 \\
\hline n-pentane & 196 & 989 & 97 & 674 & 61.7 & 61.9 \\
\hline iso-pentane & 210 & 1039 & 104 & 716 & 92.3 & 90.3 \\
\hline neo-pentane & 148 & 789 & 98 & 666 & 85.5 & 80.2 \\
\hline 2-methyl-pentane & 325 & 1643 & 127 & 841 & 73.4 & 73.5 \\
\hline n-heptane & 360 & 1817 & 114 & 783 & 0 & 0 \\
\hline iso-octane & 351 & 1684 & 156 & 958 & 100 & 100 \\
\hline n-decane & 530 & 3834 & 145 & 966 & $<0$ & $<0$ \\
\hline
\end{tabular}


Table 3

Summary of the experimental results concerning the autoignition of alkanes from $C_{4}$ to $C_{6}$ below $900 \mathrm{~K}$.

\begin{tabular}{|c|c|c|c|c|c|}
\hline Compounds & $\begin{array}{l}\text { Type of } \\
\text { reactor }^{\mathrm{a}}\end{array}$ & $\begin{array}{c}\text { Temperature } \\
\text { Range }(\mathrm{K})\end{array}$ & $\begin{array}{c}\text { Pressure } \\
\text { Range (bar) }\end{array}$ & $\begin{array}{l}\text { Equivalence } \\
\text { ratio range }\end{array}$ & References \\
\hline \multirow{2}{*}{ n-butane } & $\mathrm{RCM}$ & $700-900$ & $8.9-11.5$ & 1 & $\begin{array}{l}\text { Carlier et al. } \\
\text { (1994) [108] }\end{array}$ \\
\hline & $\mathrm{CV}$ & $550-800$ & $1-6$ & $0.06-0.66$ & $\begin{array}{c}\text { Chandraratna and } \\
\text { Griffiths (1994) [109 }\end{array}$ \\
\hline \multirow{3}{*}{ n-pentane } & \multirow{2}{*}{$\mathrm{RCM}$} & $600-900$ & $6-11$ & 1 & $\begin{array}{l}\text { Minetti et al. } \\
\text { (1996) [110] }\end{array}$ \\
\hline & & $650-900$ & $6-9$ & 1 & $\begin{array}{c}\text { Griffiths et al. } \\
(1993,97)[111-112]\end{array}$ \\
\hline & ST & $867-1534$ & $11-530$ & 0.5 & $\begin{array}{l}\text { Zhukov et al. } \\
(2005) \text { [113] }\end{array}$ \\
\hline Iso-butane & $\mathrm{RCM}$ & 900 & $7.5-9$ & 1 & $\begin{array}{l}\text { Griffiths et al. } \\
\text { (1997) [112] }\end{array}$ \\
\hline Iso-pentane & $\mathrm{RCM}$ & $680-900$ & $8-11$ & 1 & $\begin{array}{l}\text { Minetti et al. } \\
\text { (1996) [110] }\end{array}$ \\
\hline \multirow{2}{*}{ Neo-pentane } & \multirow{2}{*}{$\mathrm{RCM}$} & $680-950$ & $4-11$ & 1 & $\begin{array}{l}\text { Minetti et al. } \\
(1996)[110]\end{array}$ \\
\hline & & $750-900$ & $7.5-9$ & 1 & $\begin{array}{c}\text { Griffiths et al. } \\
\text { (1997) [112] }\end{array}$ \\
\hline Isomers of hexane & $\mathrm{RCM}$ & $680-950$ & $6-9$ & 1 & $\begin{array}{c}\text { Griffiths et al. } \\
(1993,97)[111-112]\end{array}$ \\
\hline n-hexane & $\mathrm{ST}$ & $822-1380$ & $56-244$ & 0.5 & $\begin{array}{l}\text { Zhukov et al. } \\
\text { (2004) [114] }\end{array}$ \\
\hline
\end{tabular}


Table 4

Summary of the experimental results concerning the autoignition of alkanes from $C_{7}$ below $900 \mathrm{~K}$.

\begin{tabular}{|c|c|c|c|c|c|}
\hline Compounds & $\begin{array}{l}\text { Type of } \\
\text { reactor }^{\mathrm{a}}\end{array}$ & $\begin{array}{c}\text { Temperature } \\
\text { Range }(\mathrm{K})\end{array}$ & $\begin{array}{c}\text { Pressure } \\
\text { Range (bar) }\end{array}$ & $\begin{array}{l}\text { Equivalence } \\
\text { ratio range }\end{array}$ & References \\
\hline \multirow{3}{*}{ Isomers of heptane } & \multirow{3}{*}{$\mathrm{RCM}$} & $700-950$ & $7.5-9$ & 1 & $\begin{array}{c}\text { Griffiths et al. } \\
\text { (1997) [112] }\end{array}$ \\
\hline & & $685-868$ & $7.5-9$ & 1 & $\begin{array}{l}\text { Westbrook et al. } \\
(2002)[72]\end{array}$ \\
\hline & & $640-960$ & 15 & 1 & $\begin{array}{l}\text { Silke et al. } \\
(2005)[115]\end{array}$ \\
\hline \multirow{6}{*}{ n-heptane } & \multirow{3}{*}{$\mathrm{RCM}$} & $600-900$ & $6-11$ & 1 & $\begin{array}{l}\text { Minetti et al. } \\
(1995) \text { [116] }\end{array}$ \\
\hline & & $700-960$ & $6-9$ & 1 & $\begin{array}{c}\text { Griffiths et al. } \\
(1993,97)[111-112]\end{array}$ \\
\hline & & $798-878$ & $40-44$ & $0.2-0.5$ & $\begin{array}{l}\text { Tanaka et al. } \\
\text { (2003) [117] }\end{array}$ \\
\hline & \multirow{3}{*}{$\mathrm{ST}$} & $650-1200$ & $3.2-42$ & $0.5-3$ & $\begin{array}{c}\text { Adomeit et al. (1993) } \\
{[118-119]}\end{array}$ \\
\hline & & $720-1100$ & 50 & $0.1-0.4$ & $\begin{array}{l}\text { Herzler et al. } \\
(2005)[120]\end{array}$ \\
\hline & & $850-1280$ & $15-60$ & $0.5-2$ & $\begin{array}{c}\text { Gauthier et al. } \\
(2004) \text { [121] }\end{array}$ \\
\hline \multirow{6}{*}{ Iso-octane } & \multirow{4}{*}{$\mathrm{RCM}$} & $600-900$ & $6-11$ & 1 & $\begin{array}{l}\text { Minetti et al. } \\
(1996) \text { [122] }\end{array}$ \\
\hline & & $750-900$ & $7.5-9$ & 1 & $\begin{array}{c}\text { Griffiths et al. } \\
\text { (1997) [112] }\end{array}$ \\
\hline & & $798-878$ & $40-44$ & $0.2-0.5$ & $\begin{array}{l}\text { Tanaka et al. } \\
\text { (2003) [117] }\end{array}$ \\
\hline & & $900-1020$ & $8.5-16.6$ & $0.25-1.98$ & $\begin{array}{c}\text { He et al. } \\
(2005,0,6,07) \\
{[123-125]}\end{array}$ \\
\hline & \multirow{2}{*}{$\mathrm{ST}$} & $650-1200$ & $13-45$ & 1 & $\begin{array}{c}\text { Fieweger et al. } \\
\text { (1997) [119] }\end{array}$ \\
\hline & & $855-1269$ & $14-59$ & $0.5-1$ & $\begin{array}{c}\text { Davidson et al. } \\
(2005) \text { [126] }\end{array}$ \\
\hline \multirow{3}{*}{ n-decane } & \multirow{2}{*}{$\mathrm{ST}$} & $650-1200$ & $12-50$ & $1-2$ & $\begin{array}{l}\text { Pfahl et al. } \\
(1996) \text { [127] }\end{array}$ \\
\hline & & $800-1100$ & $12-80$ & $0.5-1$ & $\begin{array}{l}\text { Zhukov et al. } \\
(2005) \text { [128] }\end{array}$ \\
\hline & $\mathrm{RCM}$ & $630-706$ & $7-30$ & 0.8 & $\begin{array}{l}\text { Kumar et al. } \\
\text { (2007) [129] }\end{array}$ \\
\hline
\end{tabular}


Table 5

Summary of the experimental results concerning the oxidation of alkanes from $C_{4}$ in continuous reactors below $900 \mathrm{~K}$.

\begin{tabular}{|c|c|c|c|c|c|}
\hline Compounds & $\begin{array}{l}\text { Type of } \\
\text { reactor }^{\mathrm{a}}\end{array}$ & $\begin{array}{l}\text { Temperature } \\
\text { range }(\mathrm{K})\end{array}$ & $\begin{array}{c}\text { Pressure } \\
\text { range (bar) }\end{array}$ & $\begin{array}{l}\text { Equivalence } \\
\text { ratio range }\end{array}$ & References \\
\hline \multirow[b]{2}{*}{ Neo-pentane } & FR & $620-810$ & 8 & 0.3 & $\begin{array}{l}\text { Wang et al. } \\
\text { (1999) [68] }\end{array}$ \\
\hline & JSR & $800-1230$ & $1-10$ & $0.25-2$ & $\begin{array}{c}\text { Dagaut and } \\
\text { Cathonnet (1999) } \\
{[130]}\end{array}$ \\
\hline \multirow{3}{*}{ n-heptane } & JSR & $550-1150$ & $1-40$ & $0.3-1.5$ & $\begin{array}{c}\text { Dagaut et al. } \\
\text { (1994) [131-132] }\end{array}$ \\
\hline & & $550-750$ & $2-10$ & 1 & $\begin{array}{c}\text { Ciajolo et al. } \\
(1993,98) \\
{[133-135]}\end{array}$ \\
\hline & FR & $550-850$ & 12.5 & 1 & $\begin{array}{c}\text { Callahan et al. } \\
\text { (1996) [136] }\end{array}$ \\
\hline \multirow{3}{*}{ Iso-octane } & JSR & $550-1100$ & 10 & $0.3-1.5$ & $\begin{array}{c}\text { Dagaut et al. (1994, } \\
1995) \text { [132] }\end{array}$ \\
\hline & & $550-750$ & $2-10$ & 1 & $\begin{array}{c}\text { Ciajolo et al. } \\
(1993,98) \\
{[134-135]}\end{array}$ \\
\hline & FR & $600-850$ & 12.5 & 1 & $\begin{array}{c}\text { Callahan et al. } \\
\text { (1996) [136] }\end{array}$ \\
\hline \multirow{2}{*}{ n-decane } & JSR & $550-1100$ & 10 & $0.3-1.5$ & $\begin{array}{l}\text { Dagaut et al. } \\
(1994) \text { [137] }\end{array}$ \\
\hline & FR & $600-800$ & 8 & 0.3 & $\begin{array}{l}\text { Cernansky et al. } \\
\text { (2007) [138-139] }\end{array}$ \\
\hline n-dodecane & FR & $600-800$ & 8 & $0.2-0.3$ & $\begin{array}{c}\text { Cernansky et al. } \\
(2004)(2005) \\
{[140-141]}\end{array}$ \\
\hline n-tetradecane & FR & $500-950$ & 3 & 1 & $\begin{array}{c}\text { Cavaliere et al. } \\
\text { (1993) [133] }\end{array}$ \\
\hline
\end{tabular}


Table 6

Summary of the experimental results concerning the autoignition and oxidation of ethers and esters from $\mathrm{C}_{4}$ below $900 \mathrm{~K}$.

\begin{tabular}{|c|c|c|c|c|c|}
\hline Compounds & $\begin{array}{l}\text { Type of } \\
\text { reactor }^{\mathrm{a}}\end{array}$ & $\begin{array}{c}\text { Temperature } \\
\text { range }(\mathrm{K})\end{array}$ & $\begin{array}{c}\text { Pressure } \\
\text { range (bar) }\end{array}$ & $\begin{array}{l}\text { Equivalence } \\
\text { ratio range }\end{array}$ & References \\
\hline \multirow{3}{*}{ MTBE } & ST & $750-1200$ & $13-40$ & 1 & $\begin{array}{c}\text { Fieweger et al. } \\
(1997) \text { [119] }\end{array}$ \\
\hline & \multirow{2}{*}{ JSR } & $800-1150$ & 10 & $0.5-2$ & $\begin{array}{l}\text { Glaude et al. } \\
(2000) \text { [107] }\end{array}$ \\
\hline & & $450-750$ & 7 & 1 & $\begin{array}{l}\text { Ciajolo et al. } \\
\text { (1997)[156] }\end{array}$ \\
\hline \multirow[b]{2}{*}{ ETBE } & JSR & $800-1150$ & 10 & $0.5-2$ & $\begin{array}{l}\text { Glaude et al. } \\
\text { (2000) [107] }\end{array}$ \\
\hline & $\mathrm{CV}$ & $573-873$ & $0.09-0.52$ & 9 & $\begin{array}{c}\text { El Kadi and } \\
\text { Baronnet (1995) } \\
{[54]}\end{array}$ \\
\hline DIPE & JSR & $800-1150$ & 10 & $0.5-2$ & $\begin{array}{c}\text { Goldaniga et al. } \\
1998)[98]\end{array}$ \\
\hline \multirow[t]{2}{*}{ TAME } & $\mathrm{CV}$ & $573-873$ & $0.09-0.52$ & 9 & $\begin{array}{c}\text { El Kadi and } \\
\text { Baronnet (1995) } \\
{[54]}\end{array}$ \\
\hline & JSR & $800-1150$ & 100 & $0.5-2$ & $\begin{array}{c}\text { Goldaniga et al. } \\
1998)[98]\end{array}$ \\
\hline \multirow[b]{2}{*}{ Methyl butanoate } & FR & $500-900$ & 12.6 & $0.35-1.5$ & $\begin{array}{l}\text { Gaïl et al. } \\
\text { (2007) [80] }\end{array}$ \\
\hline & JSR & $800-1300$ & 1 & $1-1.13$ & $\begin{array}{c}\text { Gaïl et al. } \\
(2007) \text { [80] } \\
\text { Sarathy et al. } \\
(2007) \text { [157] }\end{array}$ \\
\hline $\begin{array}{l}\text { Methyl crotonate } \\
\text { (2-butenoate) }\end{array}$ & JSR & $800-1300$ & 1 & 1 & $\begin{array}{l}\text { Sarathy et al. } \\
(2007) \text { [157] }\end{array}$ \\
\hline
\end{tabular}




\section{Table 7}

\section{Comparison between the classes of reactions included in the mechanisms of Livermore [70], Milano [96] and Nancy [24].}

\begin{tabular}{|c|c|c|c|}
\hline Class of reaction & Models of Livermore & Models of Milano & Models of Nancy \\
\hline \multicolumn{4}{|l|}{$\begin{array}{c}\text { Primary mechanism } \\
\text { High temperature }\end{array}$} \\
\hline Unimolecular initiation & yes & yes & yes \\
\hline $\begin{array}{c}\mathrm{H} \text {-abstraction by } \mathrm{O}_{2} \text { and } \\
\text { small radicals }\end{array}$ & yes & yes & yes \\
\hline $\begin{array}{c}\text { Decomposition and } \\
\text { isomerization alkyl }(\mathrm{R} \bullet) \\
\text { radicals }\end{array}$ & yes & yes & yes \\
\hline \multicolumn{4}{|l|}{ Low temperature } \\
\hline $\begin{array}{l}\text { Oxidation of } \mathrm{R} \cdot \text { to give } \\
\text { alkene }+\cdot \mathrm{HO}_{2}\end{array}$ & $\begin{array}{l}\text { only for alkyl radicals } \\
\text { up to } \mathrm{C}_{4}\end{array}$ & for all alkyl radicals & for all alkyl radicals \\
\hline $\begin{array}{c}\text { Addition of } \mathrm{O}_{2} \text { to } \mathrm{R} \cdot \text { and } \\
\cdot \mathrm{QOOH}\end{array}$ & yes & yes & yes \\
\hline Isomerization of $\mathrm{RO}_{2} \bullet$ & yes & yes & yes \\
\hline $\begin{array}{l}\text { Disproportionation of } \\
\mathrm{RO}_{2} \bullet\end{array}$ & $\begin{array}{c}\text { with } \mathrm{HO}_{2} \bullet, \mathrm{CH}_{3} \mathrm{O}_{2} \bullet, \mathrm{R}^{\prime} \bullet \\
\text { and } \mathrm{R}^{\prime} \mathrm{O}_{2} \bullet \\
\text { (formation of } \mathrm{RO} \bullet \text { ) }\end{array}$ & no & only with $\cdot \mathrm{HO}_{2}$ \\
\hline Decomposition of RO• & yes & no & no \\
\hline $\mathrm{RO}_{2} \bullet+\mathrm{H}_{2} \mathrm{O}_{2}$ & yes & no & no \\
\hline $\begin{array}{l}\text { Decomposition of } \cdot \mathrm{QOOH} \\
\text { to give cyclic ethers }\end{array}$ & yes & yes & yes \\
\hline $\begin{array}{c}\text { Other decomposition of } \\
\cdot \text { QOOH to give alkene }+ \\
\cdot \mathrm{HO}_{2}\end{array}$ & $\begin{array}{l}\text { to give alkene }+\cdot \mathrm{HO}_{2} \\
\text { alkene }+ \text { carbony } 1+\cdot \mathrm{OH}\end{array}$ & $\begin{array}{l}\text { to give alkene }+\cdot \mathrm{HO}_{2}, \\
\text { alkene }+ \text { carbony } 1+\cdot \mathrm{OH}\end{array}$ & $\begin{array}{l}\text { to give alkene }+\cdot \mathrm{HO}_{2}, \mathrm{R} \bullet^{\bullet} \\
+\mathrm{ZOOH} \text {, carbonyl }+\cdot \bullet \mathrm{OH}\end{array}$ \\
\hline $\begin{array}{c}\text { Isomerization of } \\
\bullet \mathrm{O}_{2} \mathrm{QOOH}\end{array}$ & to give ketohydroperoxide & to give ketohydroperoxide & $\begin{array}{c}\text { to give ketohydroperoxide } \\
\text { or on request } \mathrm{U}(\mathrm{OOH})_{2} \\
\text { and derived reactions }\end{array}$ \\
\hline \multicolumn{4}{|l|}{ Secondary mechanism } \\
\hline $\begin{array}{l}\text { Decomposition of } \\
\text { peroxides }\end{array}$ & yes & lumped & lumped \\
\hline $\begin{array}{c}\text { H-abstraction from } \\
\text { alkenes }\end{array}$ & yes & & \\
\hline $\begin{array}{c}\text { Decomposition of alkenyl } \\
\text { radicals }\end{array}$ & yes & lumped & lumped \\
\hline $\begin{array}{l}\text { Addition of small radicals } \\
\text { to alkenes }\end{array}$ & yes & lumped & lumped \\
\hline Alkene decomposition & yes & no & no \\
\hline $\begin{array}{c}\text { H-abstraction from cyclic } \\
\text { ethers }\end{array}$ & $\begin{array}{l}\text { yes, followed by ring } \\
\text { opening }\end{array}$ & $\begin{array}{l}\text { lumped, followed by ring } \\
\text { opening }\end{array}$ & $\begin{array}{c}\text { lumped, followed by ring } \\
\text { opening or by addition to } \\
\text { oxygen }\end{array}$ \\
\hline $\begin{array}{l}\mathrm{H} \text {-abstraction from } \mathrm{C}_{4+} \\
\text { aldehydes and ketones }\end{array}$ & no & no & yes \\
\hline
\end{tabular}


Table 8

Comparison between the rate expressions of the isomerizations of peroxy radicals considered in the models of Livermore [70], Milano [92,94] and Nancy [24] and those proposed by the team of Pilling [161].

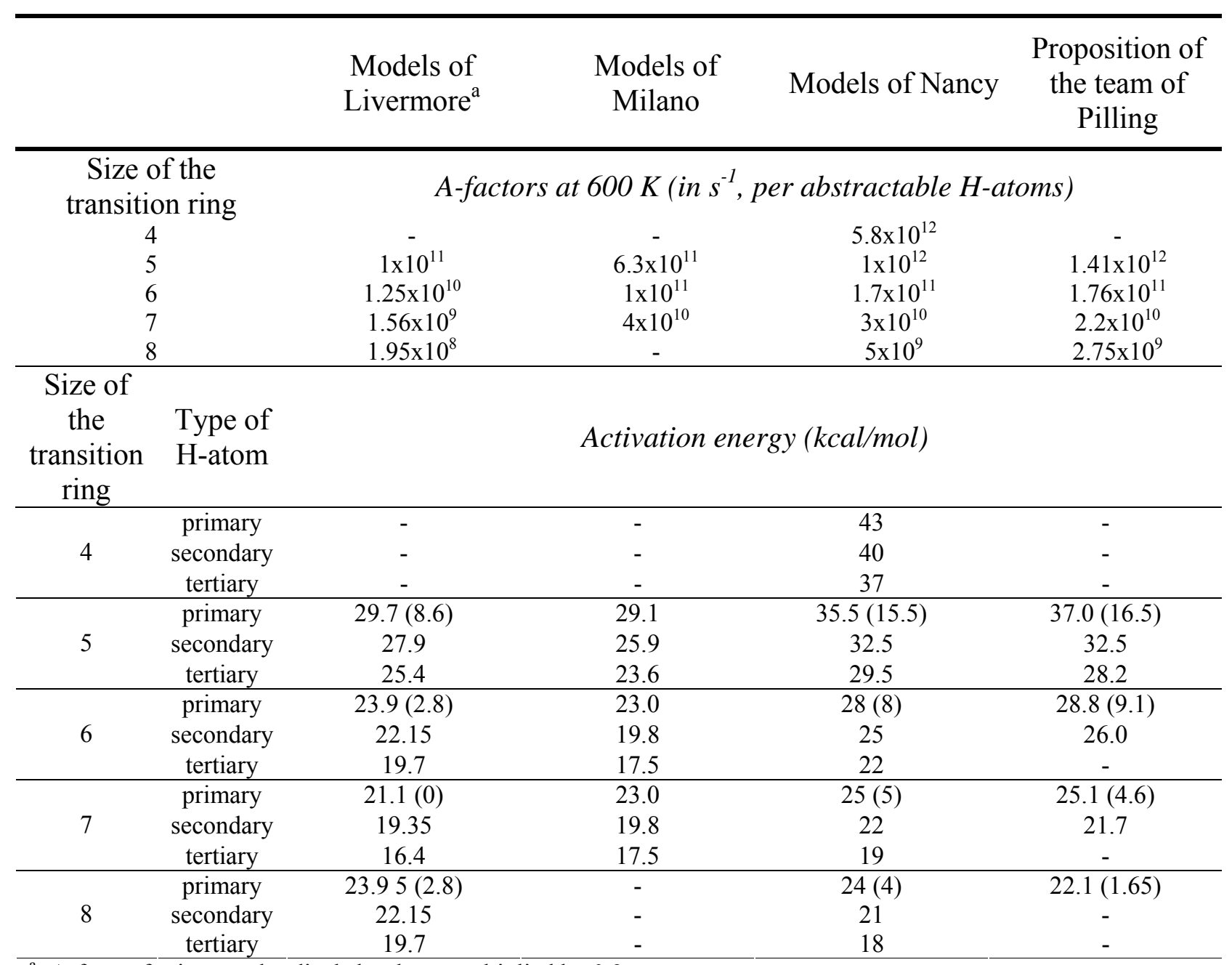

${ }^{\mathrm{a}}$ : A-factor for iso-octyl radicals has been multiplied by 0.3 .

b: The value in brackets is the ring strain energy. 


\section{Table 9}

Comparison between the rate expressions (in $\mathrm{s}^{-1}$, kcal, mol units) of the decompositions of hydroperoxyalkyl radicals to give cyclic ethers considered in the models of Livermore [70], Milano [92] and Nancy [24].

Size of the cyclic ethers Models of Livermore Models of Milano Models of Nancy

\begin{tabular}{cccccccccc}
\hline & $\mathrm{A}$ & $\mathrm{n}$ & $\mathrm{Ea}$ & $\mathrm{A}$ & $\mathrm{n}$ & $\mathrm{Ea}$ & $\mathrm{A}$ & $\mathrm{n}$ & $\mathrm{Ea}$ \\
\cline { 2 - 9 } 3 & $6 \times 10^{11}$ & 0 & 22 & $1 \times 10^{12}$ & 0 & 18 & $6 \times 10^{11}$ & 0 & 17.95 \\
4 & $7.5 \times 10^{10}$ & 0 & 15.25 & $1.6 \times 10^{11}$ & 0 & 17 & $9.1 .10^{10}$ & 0 & 16.6 \\
5 & $9.38 \times 10^{9}$ & 0 & 7 & $2.5 \times 10^{10}$ & 0 & 8.5 & $3.6 .10^{9}$ & 0 & 7 \\
6 & $1.17 \times 10^{9}$ & 0 & 1.8 & - & - & - & $1.7 .10^{8}$ & 0 & 1.95 \\
\hline
\end{tabular}




\section{Table 10}

Comparison between the rate expressions (in $\mathrm{s}^{-1}$, kcal, mol units) of the decompositions of hydroperoxyalkyl radicals to give acyclic species considered in the models of Livermore [70], Milano [94] and Nancy [24].

\begin{tabular}{|c|c|c|c|c|c|c|c|c|c|}
\hline \multirow[t]{2}{*}{ Broken bond } & \multicolumn{3}{|c|}{ Models of Livermore } & \multicolumn{3}{|c|}{ Models of Milano } & \multicolumn{3}{|c|}{ Models of Nancy } \\
\hline & A & $\mathrm{n}$ & $\mathrm{Ea}$ & A & $\mathrm{n}$ & $\mathrm{Ea}$ & A & $\mathrm{n}$ & $\mathrm{Ea}$ \\
\hline $\mathrm{C}-\mathrm{O}$ & & & $18-21.3^{\mathrm{a}}$ & $5 \times 10^{13}$ & 0 & 23 & $8 \times 10^{12}$ & 0 & 26 \\
\hline $\mathrm{C}-\mathrm{C}$ & & & $26.8-31^{\mathrm{a}}$ & $3.2 \times 10^{13}$ & 0 & 22.5 & $2 \times 10^{13}$ & 0 & $26.7-31^{b}$ \\
\hline $\mathrm{O}-\mathrm{O}$ & - & - & - & - & - & - & $1 \times 10^{9}$ & 0 & 7.5 \\
\hline
\end{tabular}

${ }^{\mathrm{a}}$ : Values calculated from the data of the reverse reaction using thermochemistry,

${ }^{\mathrm{b}}$ : Values depending on the type of radical obtained radical $\left(\mathrm{CH}_{3} \bullet: 31\right.$, primary radical: 28.7 , secondary radical: 27.7 , tertiary radical: 26.7$)$. 
Table 11

Summary of the experimental results concerning the autoignition and oxidation of alkenes from $\mathrm{C}_{4}$ below $900 \mathrm{~K}$.

\begin{tabular}{|c|c|c|c|c|c|}
\hline Compounds & $\begin{array}{l}\text { Type of } \\
\text { reactor }^{\mathrm{a}}\end{array}$ & $\begin{array}{l}\text { Temperature } \\
\text { range }(\mathrm{K})\end{array}$ & $\begin{array}{c}\text { Pressure } \\
\text { range (bar) }\end{array}$ & $\begin{array}{l}\text { Equivalence } \\
\text { ratio range }\end{array}$ & References \\
\hline \multirow[t]{2}{*}{ Iso-butene } & \multirow[t]{2}{*}{ JSR } & $800-1230$ & $1-10$ & $0.2-2$ & $\begin{array}{c}\text { Dagaut and } \\
\text { Cathonnet (1998) } \\
{[195]}\end{array}$ \\
\hline & & $833-913$ & 1 & $3-6$ & $\begin{array}{l}\text { Baugé et al. } \\
(1998)[196]\end{array}$ \\
\hline \multirow{2}{*}{ 1-pentene } & $\mathrm{RCM}$ & $600-900$ & $6.8-9.2$ & 1 & $\begin{array}{l}\text { Ribaucour et al. } \\
(2000)[77,197]\end{array}$ \\
\hline & FR & $600-800$ & 6 & 1 & $\begin{array}{l}\text { Prabhu et al. } \\
\text { (1996) [198] }\end{array}$ \\
\hline $\begin{array}{c}\text { Straight-chain isomers } \\
\text { of hexene }\end{array}$ & $\mathrm{RCM}$ & $630-850$ & $6.8-8.5$ & 1 & $\begin{array}{l}\text { Vanhove et al. } \\
(2006) \text { [199] }\end{array}$ \\
\hline 1-hexene & $\begin{array}{l}\text { JSR } \\
\end{array}$ & $750-1150$ & 10 & $0.5-1$ & $\begin{array}{l}\text { Yahyaoui et al. } \\
(2006)[200]\end{array}$ \\
\hline $\begin{array}{c}\text { Straight-chain isomers } \\
\text { of heptene }\end{array}$ & $\mathrm{RCM}$ & 827 & 41.6 & 0.4 & $\begin{array}{l}\text { Tanaka et al. } \\
(2003)[117]\end{array}$ \\
\hline
\end{tabular}




\section{Table 12}

Rate constants for the additions of small radicals to the double bond of alkenes (high pressure limits, in $\mathrm{s}^{-1}$, kcal, mol units).

\begin{tabular}{|c|c|c|c|c|c|c|c|}
\hline \multirow[t]{2}{*}{ Added radicals } & \multirow[t]{2}{*}{ References } & \multicolumn{3}{|c|}{ Addition to $=\mathrm{CH} 2$} & \multicolumn{3}{|c|}{ Addition to $=\mathrm{CH}-$} \\
\hline & & $\mathrm{A}$ & $\mathrm{n}$ & $\mathrm{Ea}$ & A & $\mathrm{n}$ & $\mathrm{Ea}$ \\
\hline$\cdot \mathrm{H}$ & $\begin{array}{c}\text { Tsang (1991) } \\
{[35]}\end{array}$ & $1.32 \times 10^{13}$ & 0 & 1.56 & $1.32 \times 10^{13}$ & 0 & 3.26 \\
\hline$\cdot \mathrm{CH}_{3}$ & $\begin{array}{c}\text { Tsang (1991) } \\
{[35]}\end{array}$ & $1.69 \times 10^{11}$ & 0 & 7.40 & $9.64 \times 10^{10}$ & 0 & 8.00 \\
\hline$\bullet \mathrm{OH}$ & $\begin{array}{c}\text { Wilk et al. (1989) } \\
\text { [191] }\end{array}$ & $1.37 \times 10^{12}$ & 0 & -1.04 & $1.37 \times 10^{12}$ & 0 & -1.04 \\
\hline$\cdot \mathrm{HO}_{2}$ & $\begin{array}{c}\text { Tsang (1991) } \\
{[35]}\end{array}$ & $1.0 \times 10^{12}$ & 0 & 14.20 & - & - & - \\
\hline
\end{tabular}


Table 13

Rate constants for the reactions of oxygen molecules with the radicals deriving from alkenes (in $\mathrm{s}^{-1}$, kcal, mol units).

Additions

\begin{tabular}{|c|c|c|c|c|c|c|c|c|c|c|c|}
\hline \multicolumn{2}{|c|}{ Initial radical } & References & \multicolumn{4}{|c|}{ A } & $\mathrm{n}$ & & \multicolumn{3}{|c|}{$\mathrm{Ea}$} \\
\hline \multicolumn{2}{|c|}{ alkylic alkenyl } & \multicolumn{10}{|c|}{ as for alkyl radicals } \\
\hline \multicolumn{2}{|c|}{ allylic alkenyl } & $\begin{array}{l}\text { Morgan et al. } \\
\text { (1982) [213] }\end{array}$ & \multicolumn{4}{|c|}{$1.2 \times 10^{10}$} & \multicolumn{2}{|l|}{0} & \multicolumn{3}{|c|}{-2.30} \\
\hline \multicolumn{2}{|c|}{ hydroxyalkyl } & \multicolumn{10}{|c|}{ as for alkyl radicals } \\
\hline \multicolumn{12}{|c|}{ Oxidations } \\
\hline & & & \multicolumn{3}{|c|}{$\begin{array}{l}\text { Abstraction of a } \\
\text { primary H-atom } \\
\text { or formation of } \\
\text { an aldehyde }\end{array}$} & \multicolumn{3}{|c|}{$\begin{array}{l}\text { Abstraction of a } \\
\text { secondary H-atom } \\
\text { or formation of an } \\
\text { ketone }\end{array}$} & \multicolumn{3}{|c|}{$\begin{array}{l}\text { Abstraction of a } \\
\text { tertiary H-atom }\end{array}$} \\
\hline $\begin{array}{l}\text { Initial } \\
\text { radical }\end{array}$ & $\begin{array}{c}\text { Abstracted } \\
\text { H-atoms }\end{array}$ & References & $\mathrm{A} \times 10^{11}$ & $\mathrm{n}$ & $\mathrm{Ea}$ & $\mathrm{A} \times 10^{11}$ & $\mathrm{n}$ & $\mathrm{Ea}$ & $\mathrm{A} \times 10^{11}$ & $\mathrm{n}$ & $\mathrm{Ea}$ \\
\hline \multirow[b]{2}{*}{ alkylic } & alkylic & $\begin{array}{l}\text { Warth et al. } \\
\text { (1998) [103] }\end{array}$ & 2.3 & 0 & 5.0 & 7.9 & 0 & 5.0 & 4.5 & 0 & 5.0 \\
\hline & allylic & $\begin{array}{c}\text { Touchard et } \\
\text { al. }(2005) \\
{[186]^{\mathrm{a}}}\end{array}$ & - & - & - & 1.3 & 0 & 2.5 & 0.75 & 0 & 2.5 \\
\hline \multirow{2}{*}{ allylic } & alkylic & $\begin{array}{c}\text { Touchard et } \\
\text { al. }(2005) \\
{[186]^{\mathrm{b}}}\end{array}$ & 2.3 & 0 & 15.2 & 7.9 & 0 & 15.2 & 7.9 & 0 & 15.2 \\
\hline & vinylic & $\begin{array}{l}\text { Stothard and } \\
\text { Walker } \\
\text { (1992) [184] }\end{array}$ & - & - & - & - & - & - & 10.0 & 0 & 22.7 \\
\hline \multirow{2}{*}{$\begin{array}{l}\text { hydroxy } \\
\text { alkyl }\end{array}$} & hydroxy & $\begin{array}{c}\text { Touchard et } \\
\text { al. }(2005) \\
{[186]^{c}}\end{array}$ & 79 & 0 & 5.0 & 7.9 & 0 & 5.0 & - & - & - \\
\hline & alkylic & $\begin{array}{l}\text { Warth et al. } \\
\text { (1998) [103] }\end{array}$ & 2.3 & 0 & 5.0 & 7.9 & 0 & 5.0 & 4.5 & 0 & 5.0 \\
\hline
\end{tabular}

a: A-factors divided by 6 , compared to those of alkyl radicals, to consider the fact that the formation of the conjugated diene from a non resonance stabilized radical involves a loss of an additional free rotation compared to the formation of an alkene from an alkyl radical; a difference of activation energy of $2.5 \mathrm{kcal} / \mathrm{mol}$ compared to alkylic $\mathrm{H}$-atoms has been taken into account.

${ }^{\mathrm{b}}$ : A-factors taken equal to those of alkyl radicals; activation energy derived from the experimental measurement of the ratio between the concentration of 1,3-pentadiene and that of 1,3-butadiene at $753 \mathrm{~K}$ by Baldwin and Walker [181] and from the values proposed by Perrin et al. (1988) [214] for the decomposition of 1-pentene-3-yl radicals to give 1,3-butadiene and methyl radicals.

c: Rate parameters deduced from the measurements of Miyoshi et al. at room temperature [215]. 


\section{Table 14}

Rate expressions (in $\mathrm{s}^{-1}$, kcal, mol units) of the formation of cyclic ethers including an unsaturated ring or a ring conjugated to a double bond [186].

\begin{tabular}{ccccc}
\hline $\begin{array}{c}\text { Size of the } \\
\text { cyclic ethers }\end{array}$ & A & n & Ea & $\begin{array}{c}\text { Difference in strain } \\
\text { energy between the } \\
\text { cycloalkane and the } \\
\text { cycloalkene [29] }\end{array}$ \\
\hline 4 & $1.4 \times 10^{11}$ & 0 & 18.9 & 3.6 \\
5 & $1.2 \times 10^{10}$ & 0 & 6.5 & -0.4 \\
6 & $8.6 .10^{8}$ & 0 & 3.2 & 1.4 \\
\hline
\end{tabular}


Table 15

Rate expressions (in $\mathrm{s}^{-1}$, kcal, mol units) of the decomposition of hydroperoxyalkenyl radicals to give acyclic species.

\begin{tabular}{cccccc}
\hline Broken bond & $\begin{array}{c}\text { Type of initial } \\
\text { radical }\end{array}$ & $\begin{array}{c}\text { Obtained } \\
\text { products }\end{array}$ & $\mathrm{A}$ & $\mathrm{n}$ & $\mathrm{Ea}$ \\
\hline $\mathrm{C}-\mathrm{O}$ & Allylic & Diene $+\mathrm{HO}_{2}{ }^{\bullet}$ & $8 \times 10^{12}$ & 0 & $33.2^{\mathrm{a}}$ \\
\hline $\mathrm{C}-\mathrm{C}$ & Allylic & $\begin{array}{c}\text { Diene }+ \\
\text { hydroperoxyalkyl } \\
\text { radical }\end{array}$ & $1.3 \times 10^{13}$ & 0 & $33.9-35.9^{\mathrm{b}}$ \\
\cline { 2 - 6 } & Alkylic & $\begin{array}{c}\text { Allylic radical }+ \\
\text { hydroperoxyalkene }\end{array}$ & $2 \times 10^{13}$ & 0 & $22.5^{\mathrm{c}}$ \\
\hline $\mathrm{O}-\mathrm{O}$ & Allylic & $\begin{array}{c}\text { Unsaturated } \\
\text { aldehyde or ketone }+ \\
\text { OH• }\end{array}$ & $1 \times 10^{9}$ & 0 & $14.7^{\mathrm{a}}$ \\
\hline
\end{tabular}

\footnotetext{
${ }^{\mathrm{a}}$ : Values deduced from those proposed for hydroperoxyalkyl radicals with an activation energy $7.2 \mathrm{kcal} / \mathrm{mol}$ higher accounting for the equal difference between allylic and alkyl radicals as for the breaking of a C-C bond.

b: Values depending on the type of radical hydroperoxyalkyl (primary radical: 35.9, secondary radical: 34.9, tertiary radical: 33.9) and deduced from the values proposed by Perrin et al. (1988) [214] for the decomposition of 1-pentene-3-yl radicals to give 1,3-butadiene and methyl radicals and from the values used for hydroperoxyalkyl radicals.

${ }^{c}$ : Values deduced from those proposed for the decomposition of 1-pentene-5-yl radicals to give ethylene and allyl radicals proposed by Gierczak et al. (1986) [216].
} 
Table 16

Summary of the experimental results concerning the autoignition and oxidation of cycloalkanes from $\mathrm{C}_{4}$ below $1000 \mathrm{~K}$.

\begin{tabular}{|c|c|c|c|c|c|}
\hline Compounds & $\begin{array}{l}\text { Type of } \\
\text { reactor }^{\mathrm{a}}\end{array}$ & $\begin{array}{c}\text { Temperature } \\
\text { range }(\mathrm{K})\end{array}$ & $\begin{array}{l}\text { Pressure } \\
\text { range (bar) }\end{array}$ & $\begin{array}{l}\text { Equivalence } \\
\text { ratio range }\end{array}$ & References \\
\hline \multirow{4}{*}{ Cyclohexane } & \multirow{2}{*}{$\mathrm{RCM}$} & $600-900$ & $7-14$ & 1 & $\begin{array}{l}\text { Lemaire et al. } \\
\text { (2001) [229] }\end{array}$ \\
\hline & & 827 & 41.6 & 0.4 & $\begin{array}{l}\text { Tanaka et al. } \\
\text { (2003) [117] }\end{array}$ \\
\hline & JSR & $750-1200$ & $1-10$ & $0.5-1.5$ & $\begin{array}{c}\text { Voisin et al. } \\
\text { (1992)[230] } \\
\text { Dagaut } \\
\text { (2002)[231] }\end{array}$ \\
\hline & $\mathrm{CV}$ & 635 & 0.059 & 9 & $\begin{array}{l}\text { Klaï et al. } \\
\text { (1993)[222] }\end{array}$ \\
\hline \multirow{3}{*}{ Methylcyclohexane } & \multirow{2}{*}{$\mathrm{RCM}$} & $680-980$ & $10-20$ & 1 & $\begin{array}{l}\text { Pitz et al. } \\
\text { (2007) [225] }\end{array}$ \\
\hline & & 827 & 41.6 & 0.4 & $\begin{array}{l}\text { Tanaka et al. } \\
(2003) \text { [117] }\end{array}$ \\
\hline & ST & $795-1560$ & $1-50$ & $0.5-2$ & $\begin{array}{l}\text { Vasu et al. } \\
(2007)[232]\end{array}$ \\
\hline n-propylcyclohexane & JSR & $950-1200$ & 1 & 1 & $\begin{array}{c}\text { Dagaut } \\
(2002)[231]\end{array}$ \\
\hline n-butylcyclohexane & FR & $600-800$ & 8 & 0.3 & $\begin{array}{l}\text { Natelson et al. } \\
(2007) \text { [138] }\end{array}$ \\
\hline
\end{tabular}

${ }^{\mathrm{a}}$ : see nomenclature. 


\section{Table 17}

Comparison between the rate expressions of the isomerizations of cyclic peroxy radicals considered in the models of Livermore [224,225], Milano [227] and Nancy [228] and those proposed by Handford-styring and Walker [235].

\begin{tabular}{|c|c|c|c|c|c|}
\hline & & $\begin{array}{l}\text { Models of } \\
\text { Livermore }^{\mathrm{a}}\end{array}$ & $\begin{array}{l}\text { Model of } \\
\text { Milano }\end{array}$ & $\begin{array}{l}\text { Model of } \\
\text { Nancy }\end{array}$ & $\begin{array}{l}\text { Estimation } \\
\text { of the team } \\
\text { of Walker }\end{array}$ \\
\hline $\begin{array}{l}\text { Size of } \\
\text { the } \\
\text { transition } \\
\text { ring }\end{array}$ & $\begin{array}{l}\text { Structure of the } \\
\text { transition state } \\
\text { in the case of } \\
\text { cyclohexane }\end{array}$ & \multicolumn{4}{|c|}{ A-factors at $600 \mathrm{~K}$ (in s-1, per abstractable H-atoms) } \\
\hline 4 & & - & - & $5.8 \times 10^{12}$ & $\begin{array}{cc}- \\
-\end{array}$ \\
\hline 5 & & $\begin{array}{l}1.2 \times 10^{11} / \\
6.2 \times 10^{10}\end{array}$ & $5.5 \times 10^{11}$ & $5.8 \times 10^{12}$ & $8.71 \times 10^{11}$ \\
\hline 6 & & $4.63 \times 10^{10}$ & $3.0 \times 10^{11}$ & $5.8 \times 10^{12}$ & $6.46 \times 10^{11}$ \\
\hline $7^{\mathrm{b}}$ & & $5.5 \times 10^{9}$ & $4.1 \times 10^{11}$ & $2.9 \times 10^{10}$ & $7.59 \times 10^{10}$ \\
\hline \multicolumn{2}{|c|}{ Size of the transition ring } & \multicolumn{4}{|c|}{ Activation energy $(\mathrm{kcal} / \mathrm{mol})$} \\
\hline & 4 & - & - & 35.0 & - \\
\hline & 5 & $31.0 / 27.5(29.7)^{\mathrm{c}}$ & 32.0 (25.9) & $35.3(32.5)$ & 32.4 \\
\hline & 6 & $24.07(22.15)$ & $24.4(19.8)$ & $32.8(25)$ & 29.5 \\
\hline & 7 & 24.35 (19.35) & $29.3(19.8)$ & $25.5(22)$ & 26.8 \\
\hline \multicolumn{2}{|c|}{ Size of the transition ring } & \multicolumn{4}{|c|}{ Rate constant at $753 \mathrm{~K}_{\left(\text {in s }^{-1}\right) \text { for cyclohexane }}$} \\
\hline & 4 & - & - & $5.1 \times 10^{2}$ & - \\
\hline & 5 & $4.8 \times 10^{2}$ & $2.8 \times 10^{2}$ & $1.7 \times 10^{3}$ & $1.4 \times 10^{3}$ \\
\hline & 6 & $1.9 \times 10^{4}$ & $1.3 \times 10^{5}$ & $8.8 \times 10^{3}$ & $7.1 \times 10^{3}$ \\
\hline & 7 & $9.4 \times 10^{2}$ & $2.5 \times 10^{3}$ & $2.9 \times 10^{3}$ & $2.3 \times 10^{3}$ \\
\hline
\end{tabular}

\footnotetext{
${ }^{\mathrm{a}}$ : Value for cyclohexane [224]/value for methyl cyclohexane [225], when they are different.

b: See figure 24.

${ }^{c}$ : The value in parenthesis is for acyclic alkanes (Table 8).
} 


\section{Table 18}

Comparison between the rate expressions (in $\mathrm{s}^{-1}$, kcal, mol units) of the formation of bicyclic ethers deriving from cyclohexane considered in the models of Livermore [224], Milano [227] and Nancy [228].

Size of the cyclic ethers Model of Livermore Model of Milano Model of Nancy

\begin{tabular}{cccccccccc}
\hline & $\mathrm{A}$ & $\mathrm{n}$ & $\mathrm{Ea}$ & $\mathrm{A}$ & $\mathrm{n}$ & $\mathrm{Ea}$ & $\mathrm{A}$ & $\mathrm{n}$ & $\mathrm{Ea}$ \\
\cline { 2 - 9 } 3 & $5.8 \times 10^{12}$ & 0 & 13.4 & $2.9 \times 10^{12}$ & 0 & 15.4 & $2.06 \times 10^{13}$ & 0 & 9.68 \\
4 & $1.4 \times 10^{12}$ & 0 & 20.0 & $1.4 \times 10^{12}$ & 0 & 23.4 & $2.06 \times 10^{13}$ & 0 & $19.6^{\mathrm{a}}$ \\
5 & $8.6 \times 10^{12}$ & 0 & 18.5 & $8.6 \times 10^{12}$ & 0 & 20.7 & $2.06 \times 10^{13}$ & 0 & 17.1 \\
\hline
\end{tabular}

${ }^{\mathrm{a}}$ : The direct formation of 1-hexenal is assumed. 
Table 19: Summary of the experimental results concerning the autoignition and oxidation of benzene and toluene below $1200 \mathrm{~K}$.

\begin{tabular}{|c|c|c|c|c|c|}
\hline Compounds & $\begin{array}{l}\text { Type of } \\
\text { reactor }^{\mathrm{a}}\end{array}$ & $\begin{array}{c}\text { Temperature } \\
\text { range }(\mathrm{K})\end{array}$ & $\begin{array}{l}\text { Pressure } \\
\text { range (bar) }\end{array}$ & $\begin{array}{c}\text { Equivalence } \\
\text { ratio range }\end{array}$ & References \\
\hline \multirow{7}{*}{ Benzene } & $\mathrm{RCM}$ & $920-1100$ & $25-45$ & $0.5-1$ & $\begin{array}{l}\text { Mittal et al. } \\
(2007)[275]\end{array}$ \\
\hline & \multirow{3}{*}{ JSR } & $900-1300$ & 0.46 & 0.1961 .02 & $\begin{array}{c}\text { Chai and Pferfferle } \\
\text { (1998) [276] }\end{array}$ \\
\hline & & $950-1350$ & $1-10$ & $0.3-1.5$ & $\begin{array}{c}\text { Ristori et al. } \\
(2001)[255] \\
\text { Marchal (1997) } \\
{[277]}\end{array}$ \\
\hline & & 923 & 1 & $1.9-3.6$ & $\begin{array}{l}\text { Da Costa et al. } \\
(2003)[256]\end{array}$ \\
\hline & \multirow{2}{*}{ FR } & 1100 & 1 & $0.76-1.3$ & $\begin{array}{l}\text { Lovell et al. } \\
(1988)[240]\end{array}$ \\
\hline & & $900-1450$ & 1 & $0.0016-0.96$ & $\begin{array}{l}\text { Alzueta et al. } \\
(2000) \text { [254] }\end{array}$ \\
\hline & $\begin{array}{c}\text { FR } \\
\text { in the flue gas } \\
\text { of a methane } \\
\text { flame }\end{array}$ & $850-960 \mathrm{~K}$ & 1 & $0.1-1.3$ & $\begin{array}{l}\text { Schöbel-Ostertag et } \\
\text { al. (2005) [257] }\end{array}$ \\
\hline \multirow{9}{*}{ Toluene } & ST & $975-1269$ & $14-59$ & $0.5-1$ & $\begin{array}{l}\text { Davidson et al. } \\
(2005)[126]\end{array}$ \\
\hline & \multirow{4}{*}{$\mathrm{RCM}$} & $850-950$ & $6-9$ & 1 & $\begin{array}{l}\text { Griffiths et al. } \\
\text { (1993) [111] }\end{array}$ \\
\hline & & 900 & $1-24$ & 1 & $\begin{array}{l}\text { Roubaud et al. } \\
(2000) \text { [277] }\end{array}$ \\
\hline & & 827 & 41.6 & 0.4 & $\begin{array}{l}\text { Tanaka et al. } \\
(2003)[117]\end{array}$ \\
\hline & & $920-1100$ & $25-45$ & $0.5-1$ & $\begin{array}{l}\text { Mittal et al. } \\
(2007) \text { [275] }\end{array}$ \\
\hline & \multirow[t]{2}{*}{ JSR } & $1000-1375$ & 1 & $0.5-1.5$ & $\begin{array}{c}\text { Marchal } \\
\text { (1997) [278] } \\
\text { Dagaut et al. } \\
\text { (2002) [231-265] }\end{array}$ \\
\hline & & $873-923$ & 1 & $0.45-0.91$ & $\begin{array}{l}\text { Bounaceur et al. } \\
(2005)[266]\end{array}$ \\
\hline & \multirow[t]{2}{*}{ FR } & 1190 & 1 & $0.69-1.33$ & $\begin{array}{c}\text { Emdee et al. (1992) } \\
\text { [247], Klotz et al. } \\
\text { (1998) [259] }\end{array}$ \\
\hline & & $850-950$ & 12.5 & 1 & $\begin{array}{l}\text { Chaos et al. } \\
(2007)[262]\end{array}$ \\
\hline
\end{tabular}

\footnotetext{
a: see nomenclature
} 
Table 20: Summary of the experimental results concerning the autoignition and oxidation of $\mathrm{C}_{7+}$ aromatic compounds below $1200 \mathrm{~K}$.

\begin{tabular}{|c|c|c|c|c|c|}
\hline Compounds & $\begin{array}{l}\text { Type of } \\
\text { reactor }^{\mathrm{a}}\end{array}$ & $\begin{array}{l}\text { Temperature } \\
\text { range }(\mathrm{K})\end{array}$ & $\begin{array}{l}\text { Pressure } \\
\text { range (bar) }\end{array}$ & $\begin{array}{l}\text { Equivalence } \\
\text { ratio range }\end{array}$ & References \\
\hline \multirow{3}{*}{ Xylenes } & $\mathrm{RCM}$ & $600-900$ & $8-24$ & 1 & $\begin{array}{c}\text { Roubaud et al. } \\
(2000) \text { [244-277] }\end{array}$ \\
\hline & FR & 1093-1199 & 1 & $0.47-1.7$ & $\begin{array}{c}\text { Emdee et al. (1990, } \\
91)[259-260]\end{array}$ \\
\hline & JSR & $900-1300$ & 1 & $0.3-1.5$ & $\begin{array}{c}\text { Gail et al. (2005) } \\
{[247-268,279}\end{array}$ \\
\hline \multirow{2}{*}{ Ethylbenzene } & $\mathrm{RCM}$ & $600-900$ & $8-24$ & 1 & $\begin{array}{l}\text { Roubaud et al. } \\
(2000) \text { [277] }\end{array}$ \\
\hline & FR & 1060 & 1 & $0.64-1.3$ & $\begin{array}{l}\text { Litzinger et al. } \\
\text { (1986) [280] }\end{array}$ \\
\hline Styrene & FR & 1060 & 1 & 0.56 & $\begin{array}{c}\text { Litzinger et al. } \\
(1986) \text { [280] }\end{array}$ \\
\hline Trimethylbenzenes & $\mathrm{RCM}$ & $600-900$ & $8-24$ & 1 & $\begin{array}{l}\text { Roubaud et al. } \\
(2000) \text { [277] }\end{array}$ \\
\hline \multirow{3}{*}{ n-propylbenzene } & $\mathrm{RCM}$ & $600-900$ & $8-24$ & 1 & $\begin{array}{l}\text { Roubaud et al. } \\
(2000) \text { [277] }\end{array}$ \\
\hline & FR & 1060 & 1 & $0.65-1.5$ & $\begin{array}{c}\text { Litzinger et al. } \\
(1986)[281]\end{array}$ \\
\hline & JSR & $900-1250$ & 1 & $0.5-1.5$ & $\begin{array}{l}\text { Dagaut et al. } \\
(2002) \text { [269] }\end{array}$ \\
\hline Ethyltoluenes & $\mathrm{RCM}$ & $600-900$ & $14-19$ & 1 & $\begin{array}{l}\text { Roubaud et al. } \\
(2000) \text { [244] }\end{array}$ \\
\hline \multirow[t]{2}{*}{ n-butylbenzene } & $\mathrm{RCM}$ & $600-960$ & $8-24$ & $0.3-1$ & $\begin{array}{l}\text { Roubaud et al. } \\
\text { (2000) [244-277] } \\
\text { Ribaucour et al. } \\
(2000)[273] \\
\text { Crochet et al. } \\
(2007)[282] \\
\end{array}$ \\
\hline & FR & 1069 & 1 & 0.98 & $\begin{array}{l}\text { Brezinsky et al. } \\
\text { (1986) [283] }\end{array}$ \\
\hline \multirow{3}{*}{ 1-methylnaphthalene } & ST & $900-1500$ & 13 & 1 & $\begin{array}{l}\text { Pfahl et al. } \\
\text { (1996) [127] }\end{array}$ \\
\hline & FR & 1170 & 1 & $0.6-1.5$ & $\begin{array}{l}\text { Shaddix et al. } \\
\text { (1992) [284] }\end{array}$ \\
\hline & JSR & $800-1150$ & 10 & 1 & $\begin{array}{c}\text { Marchal } \\
\text { (1997) }[278]\end{array}$ \\
\hline Indane & JSR & $950-1350$ & 1 & $0.5-1$ & $\begin{array}{l}\text { Dagaut et al. } \\
\text { (2001) [285] }\end{array}$ \\
\hline
\end{tabular}

\footnotetext{
${ }^{\mathrm{a}}$ : see nomenclature.
} 
Table 21: Summary of the experimental results concerning the autoignition and oxidation of binary mixtures containing only saturated compounds from $\mathrm{C}_{4}$ below $900 \mathrm{~K}$.

\begin{tabular}{|c|c|c|c|c|c|}
\hline Compounds & $\begin{array}{l}\text { Type of } \\
\text { reactor }^{\mathrm{a}}\end{array}$ & $\begin{array}{l}\text { Temperature } \\
\text { range }(\mathrm{K})\end{array}$ & $\begin{array}{c}\text { Pressure } \\
\text { range (bar) }\end{array}$ & $\begin{array}{l}\text { Equivalence } \\
\text { ratio range }\end{array}$ & References \\
\hline \multicolumn{6}{|c|}{ Mixtures of alkanes } \\
\hline \multirow{7}{*}{$\begin{array}{c}\text { Mixtures of } \\
\text { n-heptane/iso-octane } \\
(\mathrm{PRF})\end{array}$} & \multirow{3}{*}{$\mathrm{RCM}$} & $680-930$ & $6-9$ & 1 & $\begin{array}{l}\text { Griffiths et al. } \\
(1993)[111]\end{array}$ \\
\hline & & $630-910$ & $12-17$ & 1 & $\begin{array}{c}\text { Callahan et al. } \\
\text { (1996) [136] }\end{array}$ \\
\hline & & $798-878$ & $40-44$ & $0.2-0.5$ & $\begin{array}{l}\text { Tanaka et al. } \\
(2003)[117]\end{array}$ \\
\hline & \multirow{2}{*}{ ST } & $650-1200$ & $12-50$ & 1 & $\begin{array}{c}\text { Fieweger et al. } \\
\text { (1997) [119] }\end{array}$ \\
\hline & & $673-1184$ & 40 & 1 & $\begin{array}{l}\text { Hartmann et al. } \\
(2007)[326]\end{array}$ \\
\hline & JSR & $550-1100$ & 10 & $0.2-1$ & $\begin{array}{c}\text { Dagaut et al. } \\
\text { (1994) [320] } \\
\text { Dubreuil at al. } \\
\text { (2007) [22] }\end{array}$ \\
\hline & FR & $550-900$ & 12.5 & 1 & $\begin{array}{c}\text { Callahan et al. } \\
(1996) \text { [136] }\end{array}$ \\
\hline $\begin{array}{c}\text { Mixtures of } \\
\text { n-decane/iso-octane }\end{array}$ & FR & $600-800$ & 8 & 0.3 & $\begin{array}{l}\text { Kurman et al. } \\
(2007) \text { [139] }\end{array}$ \\
\hline $\begin{array}{c}\text { Mixtures of } \\
\text { n-dodecane/iso-cetane }\end{array}$ & FR & $600-800$ & 8 & 0.3 & $\begin{array}{c}\text { Cernansky et al. } \\
(2004)(2005) \\
{[140-141]} \\
\end{array}$ \\
\hline \multicolumn{6}{|c|}{ Alkane/ether mixtures } \\
\hline $\begin{array}{c}\text { Mixtures of } \\
\text { n-pentane/MTBE }\end{array}$ & $\mathrm{CV}$ & 580 & 0.059 & $7.75-8$ & $\begin{array}{c}\text { El Kadi and } \\
\text { Baronnet (1995) } \\
{[54]}\end{array}$ \\
\hline $\begin{array}{c}\text { Mixtures of } \\
\text { n-pentane/ETBE }\end{array}$ & $\mathrm{CV}$ & 580 & 0.059 & $8-8.5$ & $\begin{array}{c}\text { El Kadi and } \\
\text { Baronnet (1995) } \\
{[54]}\end{array}$ \\
\hline $\begin{array}{c}\text { Mixtures of } \\
\text { n-pentane /TAME }\end{array}$ & $\mathrm{CV}$ & 580 & 0.059 & $8-8.5$ & $\begin{array}{c}\text { El Kadi and } \\
\text { Baronnet (1995) } \\
{[54]}\end{array}$ \\
\hline $\begin{array}{c}\text { Mixture n-pentane } \\
\text { /MTBE }\end{array}$ & JSR & $570-1150$ & 10 & 1 & $\begin{array}{l}\text { Dagaut et al. } \\
\text { (1997) [321] }\end{array}$ \\
\hline $\begin{array}{c}\text { Mixtures of } \\
\text { n-heptane/ETBE }\end{array}$ & JSR & $570-1150$ & 10 & 1 & $\begin{array}{l}\text { Dagaut et al. } \\
(1997)[321] \\
\end{array}$ \\
\hline \multicolumn{6}{|c|}{ Alkane/cyclo-alkane mixtures } \\
\hline $\begin{array}{c}\text { Mixtures of } \\
\text { n-dodecane/ } \\
\text { methylcyclohexane }\end{array}$ & FR & $600-800$ & 8 & 0.3 & $\begin{array}{c}\text { Cernansky et al. } \\
(2004)(2005) \\
{[140-141]}\end{array}$ \\
\hline
\end{tabular}


Table 22: Summary of the experimental results concerning the autoignition and oxidation of binary mixtures containing unsaturated compounds from $\mathrm{C}_{4}$ below $900 \mathrm{~K}$.

\begin{tabular}{|c|c|c|c|c|c|}
\hline Compounds & $\begin{array}{l}\text { Type of } \\
\text { reactor }\end{array}$ & $\begin{array}{l}\text { Temperature } \\
\text { range }(\mathrm{K})\end{array}$ & $\begin{array}{l}\text { Pressure } \\
\text { range (bar) }\end{array}$ & $\begin{array}{c}\text { Equivalence } \\
\text { ratio range }\end{array}$ & References \\
\hline \multicolumn{6}{|c|}{ Alkane/alkene mixtures } \\
\hline \multirow{2}{*}{$\begin{array}{c}\text { Mixtures of } \\
\text { iso-octane/1-hexene }\end{array}$} & MCR & $650-900$ & $12.6-13.9$ & 1 & $\begin{array}{l}\text { Vanhove et al. } \\
(2006) \text { [314] }\end{array}$ \\
\hline & JSR & $750-1150$ & 10 & $0.5-1.5$ & $\begin{array}{c}\text { Yahyaoui } \\
(2005)[327] \\
\end{array}$ \\
\hline \multicolumn{6}{|c|}{ Aromatic/alkene mixtures } \\
\hline \multirow{2}{*}{$\begin{array}{l}\text { Mixtures of } \\
\text { toluene/1-hexene }\end{array}$} & MCR & $650-900$ & $11.8-14.8$ & 1 & $\begin{array}{l}\text { Vanhove et al. } \\
(2006) \text { [314] }\end{array}$ \\
\hline & JSR & $750-1170$ & 10 & $0.5-1.5$ & $\begin{array}{c}\text { Yahyaoui } \\
(2005)[327]\end{array}$ \\
\hline $\begin{array}{c}\text { Mixtures of } \\
\text { toluene/di-iso-butylene }\end{array}$ & MCR & $740-1150$ & $15-45$ & 0.75 & $\begin{array}{c}\text { Mittal and Sung } \\
(2007)[328]\end{array}$ \\
\hline \multicolumn{6}{|c|}{ Alkane/aromatic mixtures } \\
\hline $\begin{array}{c}\text { Mixtures of } \\
\text { n-heptane/benzene }\end{array}$ & MCR & $600-950$ & $3.3-4.9$ & 1 & $\begin{array}{l}\text { Vanhove et al. } \\
(2006)[314]\end{array}$ \\
\hline \multirow{6}{*}{$\begin{array}{c}\text { Mixtures of } \\
\text { n-heptane/toluene }\end{array}$} & ST & $620-1180$ & $10-50$ & $0.3-1$ & $\begin{array}{l}\text { Herzler et al. } \\
(2007)[301]\end{array}$ \\
\hline & \multirow{2}{*}{ MCR } & $600-950$ & $3.9-4.9$ & 1 & $\begin{array}{l}\text { Vanhove et al. } \\
\text { (2006) [314] }\end{array}$ \\
\hline & & 827 & 41.6 & 0.4 & $\begin{array}{l}\text { Tanaka et al. } \\
(2003)[117]\end{array}$ \\
\hline & \multirow{2}{*}{ JSR } & $580-640$ & 7 & 1 & $\begin{array}{c}\text { Ciajolo et D'Anna } \\
\text { (1993) [134] }\end{array}$ \\
\hline & & $550-1000$ & 10 & 0.2 & $\begin{array}{l}\text { Dubreuil at al. } \\
\text { (2007) [22] }\end{array}$ \\
\hline & FR & $550-950$ & 12.5 & 1 & $\begin{array}{l}\text { Chaos et al. } \\
(2007)[262]\end{array}$ \\
\hline \multirow{4}{*}{$\begin{array}{l}\text { Mixture iso-octane/ } \\
\text { toluene }\end{array}$} & \multirow{2}{*}{ MCR } & $600-900$ & $12.0-14.6$ & 1 & $\begin{array}{l}\text { Vanhove et al. } \\
(2006) \text { [314] }\end{array}$ \\
\hline & & $740-1150$ & $15-45$ & 0.75 & $\begin{array}{l}\text { Mittal and Sung } \\
(2007)[328]\end{array}$ \\
\hline & JSR & $750-1170$ & 10 & $0.5-1.5$ & $\begin{array}{c}\text { Yahyaoui } \\
(2005)[327]\end{array}$ \\
\hline & FR & $550-950$ & 12.5 & 1 & $\begin{array}{l}\text { Chaos et al. } \\
(2007) \text { [262] }\end{array}$ \\
\hline $\begin{array}{c}\text { Mixtures of } \\
\text { n-dodecane/ } \\
\text { 1-methylnaphthalene }\end{array}$ & FR & $600-800$ & 8 & 0.3 & $\begin{array}{c}\text { Cernansky et al. } \\
(2004)(2005) \\
{[140-141]}\end{array}$ \\
\hline
\end{tabular}


Table 23: Summary of the experimental results concerning the autoignition and oxidation of tertiary or quaternary mixtures containing compounds from $\mathrm{C}_{4}$ below $900 \mathrm{~K}$.

\begin{tabular}{|c|c|c|c|c|c|}
\hline Compounds & $\begin{array}{l}\text { Type of } \\
\text { reactor }^{\mathrm{a}}\end{array}$ & $\begin{array}{l}\text { Temperature } \\
\text { range }(\mathrm{K})\end{array}$ & $\begin{array}{l}\text { Pressure } \\
\text { range (bar) }\end{array}$ & $\begin{array}{l}\text { Equivalence } \\
\text { ratio range }\end{array}$ & References \\
\hline \multicolumn{6}{|c|}{ Alkanes/aromatic mixtures } \\
\hline \multirow{3}{*}{$\begin{array}{l}\text { Mixture n-heptane/ } \\
\text { iso-octane/toluene }\end{array}$} & ST & $850-1280$ & $15-60$ & $0.5-2$ & $\begin{array}{l}\text { Gauthier et al. } \\
(2004)[121]\end{array}$ \\
\hline & MCR & 827 & 41.6 & 0.4 & $\begin{array}{l}\text { Tanaka et al. } \\
(2003) \text { [117] }\end{array}$ \\
\hline & FR & $550-950$ & 12.5 & 1 & $\begin{array}{l}\text { Chaos et al. } \\
(2007)[262]\end{array}$ \\
\hline $\begin{array}{c}\text { Mixtures of } \\
\text { n-dodecane/ } \\
\text { 1-methylnaphthalene }\end{array}$ & FR & $600-800$ & 8 & 0.3 & $\begin{array}{c}\text { Cernansky et al. } \\
(2004)(2005) \\
{[140-141]} \\
\end{array}$ \\
\hline \multicolumn{6}{|c|}{ Alkanes/alkene/aromatic mixtures } \\
\hline \multirow{2}{*}{$\begin{array}{l}\text { Mixtures of } \\
\text { iso-octane/ } \\
\text { 1-hexene/toluene }\end{array}$} & MCR & $650-900$ & 11.4-13.9 & 1 & $\begin{array}{c}\text { Vanhove et al. } \\
(2006)[314]\end{array}$ \\
\hline & JSR & $800-1130$ & 10 & 1 & $\begin{array}{c}\text { Yahyaoui et al. } \\
\text { (2007) [329] }\end{array}$ \\
\hline $\begin{array}{c}\text { Mixtures of } \\
\text { n-heptane/iso-octane/ } \\
\text { toluene/ } \\
\text { diisobutylene } \\
\end{array}$ & ST & $690-1200$ & $10-50$ & 1 & $\begin{array}{c}\text { Fikri et al. } \\
{[330]} \\
(2007)\end{array}$ \\
\hline \multicolumn{6}{|c|}{ Alkane/alkene/ether/aromatic mixtures } \\
\hline $\begin{array}{c}\text { Mixtures of } \\
\text { iso-octane/ } \\
\text { 1-hexene/ETBE/toluene }\end{array}$ & JSR & $800-1130$ & 10 & 1 & $\begin{array}{l}\text { Yahyoui et al. } \\
\text { (2007) [329] }\end{array}$ \\
\hline \multicolumn{6}{|c|}{ Alkane/cycloalkane/ether/aromatic mixtures } \\
\hline $\begin{array}{c}\text { Mixtures of } \\
\text { n-decane/ } \\
\text { n-butylcyclohexane/ } \\
\text { n-butylbenzene }\end{array}$ & FR & $600-800$ & 8 & 0.3 & $\begin{array}{l}\text { Natelson et al. } \\
\text { (2007) [138] }\end{array}$ \\
\hline $\begin{array}{c}\text { Mixtures of } \\
\text { n-decane/ } \\
\text { n-butylcyclohexane/ } \\
\text { toluene }\end{array}$ & FR & $600-800$ & 8 & 0.3 & $\begin{array}{l}\text { Natelson et al. } \\
\text { (2007) [138] }\end{array}$ \\
\hline $\begin{array}{c}\text { Mixtures of } \\
\text { n-decane/ } \\
\text { methylcyclohexane/ } \\
\text { n-butylbenzene }\end{array}$ & FR & $600-800$ & 8 & 0.3 & $\begin{array}{l}\text { Natelson et al. } \\
\text { (2007) [138] }\end{array}$ \\
\hline $\begin{array}{c}\text { Mixtures of } \\
\text { n-decane/ } \\
\text { methylcyclohexane/ } \\
\text { toluene }\end{array}$ & FR & $600-800$ & 8 & 0.3 & $\begin{array}{l}\text { Natelson et al. } \\
\text { (2007) [138] }\end{array}$ \\
\hline
\end{tabular}


Table 24

Comparison between the rate expressions (in $\mathrm{s}^{-1}$, kcal, mol units) of the "cross term" reactions involving peroxy radicals considered in the models for mixtures of Livermore [71,316-317], Stockhom [261] and Nancy [24,203,325].

\begin{tabular}{|c|c|c|c|c|c|c|c|c|c|}
\hline & \multicolumn{3}{|c|}{ Models of Livermore } & \multicolumn{3}{|c|}{ Models of Stockhom } & \multicolumn{3}{|c|}{ Models of Nancy } \\
\hline \multicolumn{10}{|c|}{$\begin{array}{r}\mathrm{RH}+\mathrm{R}^{\prime} \mathrm{OO} \bullet=\mathrm{R}^{\prime} \mathrm{OOH}+\mathrm{R} \bullet \\
\mathrm{RH} \text { is an alkane, a methylcyclohexane or an alkene molecule, }\end{array}$} \\
\hline Type of abstracted H-atom & A & $\mathrm{n}$ & $\mathrm{Ea}$ & A & $\mathrm{n}$ & $\mathrm{Ea}$ & A & $\mathrm{n}$ & $\mathrm{Ea}$ \\
\hline Primary alkylic & $2.0-2.8 \times 10^{12}$ & 0 & 20.43 & $2.0 \times 10^{12}$ & 0 & 20.43 & $2.0 \times 10^{12}$ & 0 & 20.0 \\
\hline Secondary alkylic & $2.0-2.8 \times 10^{12}$ & 0 & 17.7 & $2.0 \times 10^{12}$ & 0 & 17.7 & $1.5 \times 10^{12}$ & 0 & 17.5 \\
\hline Tertiary alkylic & $2.8 \times 10^{12}$ & 0 & 16.0 & $2.0 \times 10^{12}$ & 0 & 16.0 & $1.5 \times 10^{12}$ & 0 & 15.0 \\
\hline Primary alkylic ${ }^{b}$ & $7.9 \times 10^{3}$ & 2.6 & 17.49 & - & - & - & - & - & - \\
\hline Secondary alkylic ${ }^{b}$ & $4.8 \times 10^{3}$ & 2.6 & 14.91 & - & - & - & - & - & - \\
\hline Tertiary alkylic ${ }^{\mathrm{b}}$ & $3.6 \times 10^{3}$ & 2.6 & 11.53 & - & - & - & - & - & - \\
\hline Secondary allylic & $1.6 \times 10^{11}$ & 0 & 14.9 & - & - & - & $5 \times 10^{11}$ & 0 & 14.55 \\
\hline \multicolumn{10}{|c|}{$\mathrm{ROO} \bullet+\mathrm{ROO}^{\prime} \bullet=\mathrm{RO} \bullet+\mathrm{RO}^{\prime} \bullet+\mathrm{O}_{2}^{\mathrm{c}}$} \\
\hline & $1.4 \times 10^{16}$ & -1.61 & 1.86 & - & - & - & - & - & - \\
\hline
\end{tabular}


Table 25

Comparison between the rate expressions (in $\mathrm{s}^{-1}$, kcal, mol units) of the "cross term" reactions considered in the models for mixtures containing toluene of Livermore [316-317], Stockhom [261] and Nancy [324].

\begin{tabular}{|c|c|c|c|c|c|c|c|c|c|}
\hline & \multicolumn{3}{|c|}{ Models of Livermore } & \multicolumn{3}{|c|}{ Models of Stockhom } & \multicolumn{3}{|c|}{ Models of Nancy } \\
\hline \multicolumn{10}{|c|}{$\mathrm{RH}+\mathrm{R}^{\prime} \bullet=\mathrm{R} \mathrm{R}^{\prime}+\mathrm{R} \bullet$} \\
\hline \multirow[t]{2}{*}{$\begin{array}{c}\text { Type of abstracted } \\
\text { H-atom } \\
\end{array}$} & A & $\mathrm{n}$ & $\mathrm{Ea}$ & A & $\mathrm{n}$ & $\mathrm{Ea}$ & A & $\mathrm{n}$ & Ea \\
\hline & \multicolumn{9}{|c|}{$\mathrm{RH}$ is an toluene molecule, $\mathrm{R}^{\prime} \bullet$ is an alkyl or allylic radical, A per abstractable $\mathrm{H}$-atom } \\
\hline Primary alkyl & - & - & - & $3.3 \times 10^{10}$ & 0 & 12 & - & - & - \\
\hline Secondary alkyl & - & - & - & $3.3 \times 10^{10}$ & 0 & 12 & - & - & - \\
\hline Tertiary alkyl & - & - & - & $3.3 \times 10^{10}$ & 0 & 12 & - & - & - \\
\hline Allylic & $1 \times 10^{11}$ & 0 & 15.5 & - & - & - & $1.6 \times 10^{12}$ & 0 & 15 \\
\hline
\end{tabular}

$\mathrm{RH}$ is an alkane or a methylcyclohexane molecule, $\mathrm{R}$ '• is an benzyl radical, A per abstractable $\mathrm{H}$-atom

\begin{tabular}{|c|c|c|c|c|c|c|c|c|c|}
\hline Primary alkyl & $1.0 \times 10^{11}$ & 0 & 20.9 & - & - & - & 1.6 & 3.3 & 19.84 \\
\hline Secondary alkyl & $0.5 \times 10^{11}$ & 0 & 17.9 & - & - & - & 1.6 & 3.3 & 18.17 \\
\hline Tertiary alkyl & $1.0 \times 10^{11}$ & 0 & 15.4 & - & - & - & 1.6 & 3.3 & 17.17 \\
\hline Primary alkyl $^{\mathrm{a}}$ & $7.55 \times 10^{-1}$ & 3.46 & 15.5 & - & - & - & - & - & - \\
\hline Secondary alkyl ${ }^{\mathrm{a}}$ & $7.55 \times 10^{-1}$ & 3.46 & 15.5 & - & - & - & - & - & - \\
\hline Tertiary alkyl $^{\mathrm{a}}$ & $3.0 \times 10^{-10}$ & 6.36 & 10.9 & - & - & - & - & - & - \\
\hline
\end{tabular}

$\mathrm{RH}$ is an alkane molecule, $\mathrm{R}^{\prime} \bullet$ is phenyl radical, A per abstractable H-atom

\begin{tabular}{|c|c|c|c|c|c|c|c|c|c|}
\hline Primary & - & - & - & $\begin{array}{c}1- \\
1.5 \times 10^{11 \mathrm{~b}}\end{array}$ & 0 & $\begin{array}{l}15.0^{-} \\
15.5^{\mathrm{b}}\end{array}$ & - & - & - \\
\hline Secondary & - & - & - & $\begin{array}{c}0.5- \\
6 \times 10^{11 \mathrm{~b}}\end{array}$ & 0 & $\begin{array}{l}10.0^{-} \\
15.5^{\mathrm{b}}\end{array}$ & - & - & - \\
\hline Tertiary & - & - & - & $1 \times 10^{11}$ & 0 & 11.0 & - & - & - \\
\hline
\end{tabular}

$\mathrm{RH}$ is an alkene molecule, $\mathrm{R}^{\prime} \bullet$ is an benzyl radical, per abstractable $\mathrm{H}$-atom ${ }^{\mathrm{c}}$

\begin{tabular}{llllllll}
\hline $1 \times 10^{11}$ & 0 & 17.0 & $1 \times 10^{11}$ & 0 & 17.0 & - & - \\
\hline \\
\hline
\end{tabular}

$\mathrm{RH}$ is toluene, A per abstractable $\mathrm{H}-$ atom $^{\mathrm{c}}$

$\begin{array}{lllllllll}3.3 \times 10^{3} & 2.5 & 12.3 & 1.3 \times 10^{11} & 0 & 14 & 1.3 \times 10^{13} & 0 & 12\end{array}$

$\mathrm{RH}$ is benzaldehyde, A per abstractable $\mathrm{H}_{-a^{2}}{ }^{\mathrm{c}}$

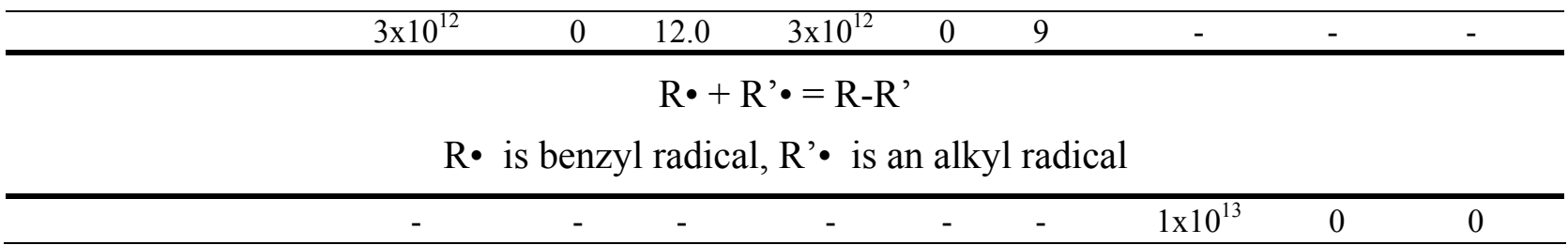

\footnotetext{
a: For methylcyclohexane.

$\mathrm{b}$ : The values depend on the radicals obtained.

': R' is methyl, ethyl, propyl and butyl in Livermore, heptyl and iso-octyl in Stockholm and methyl in Nancy.
} 


\section{FIGURES CAPTIONS}

Figure 1: Simulated influence of the fuel composition (n-heptane or n-decane/ $\alpha$-methylnaphthalene mixture) on the simulated pressure evolutions in (a) a low load direct injection diesel engine and in (b) a split injection HCCI engine [10].

Figure 2: Simplified scheme for the primary mechanism of oxidation of alkanes.

Figure 3: Schematic structure of EXGAS software.

Figure 4: Comparison between the validation of the models of Livermore [70], Milano [96] and Nancy [24] for n-heptane in a shock tube $(\phi=1$ [118], rapid compression machine data at $P_{c}$ from 3 to 4 bar [116] are also shown on the graph of Nancy). Symbols correspond to experiments and lines to simulations.

Figure 5: Comparison between the validation of the models of Livermore [70], Milano [96] and Nancy [24] for n-heptane in a jet-stirred reactor $(0.1 \% \mathrm{n}$-heptane, 10 atm, $\phi=1$, $\tau=1 \mathrm{~s}[132])$. Symbols correspond to experiments and lines to simulations.

Figure 6: Comparison between the validation of the models of Livermore [70], Milano [96] and Nancy [24] for n-heptane in a rapid compression machine $\left(\mathrm{P}_{\mathrm{c}}\right.$ from 3.7 bar to 4.6 bar , $\phi=1[116])$. Symbols correspond to experiments and lines to simulations.

Figure 7: Schematic potential energy surfaces for the reactions of n-propyl radicals with oxygen molecules according to DeSain et al. [159].

Figure 8: Isomerization by internal transfer of $\mathrm{H}$-atom from 2-heptylperoxy radicals.

Figure 9: Formation of a cyclic ether from 2-hydroperoxy-5-heptyl radicals.

Figure 10: Examples of decompositions of hydroperoxy octyl radicals.

Figure 11: Example of an isomerization involving the transfer of an $\mathrm{H}$-atom bound to a carbon atom bound to an oxygen atom.

Figure 12: Secondary reactions considered in the mechanism of Nancy for a cyclic ether 
deriving from n-heptane [162].

Figure 13: Examples of molecular reaction in the case of (a) MTBE and (b) ethyl propanoate.

Figure 14: Examples of alkenyl and hydroxyalkyl radicals illustrated in the case of 1-pentene

Figure 15: Low-temperature mechanism oxidation of alkenes exemplified by a rate of reaction analysis of the oxidation of a stoichiometric 1-pentene/air mixture at $800 \mathrm{~K}$ [186].

Figure 16: Isomerization of hydroxypentylperoxy radicals according to the mechanism of Waddington [190].

Figure 17: Validation of the models Nancy [186] for (a) 1-pentene and (b) 1-hexene using results obtained in the rapid compression machine of Lille $\left(\mathrm{P}_{\mathrm{c}}\right.$ from 6.8 bar to 10.9 bar, $\phi=1[197,199]$ ). Symbols (white dots for cool flame and black ones for autoignition) correspond to experiments and lines to simulations.

Figure 18: Comparison between the experimental (light grey) [199] and the predicted (dark grey) [203] selectivity of products during the pre-ignition period of 1-hexene under the conditions of figure $17 \mathrm{~b}\left(\mathrm{~T}_{\mathrm{c}}=707 \mathrm{~K}\right)$.

Figure 19: Unsaturated cyclic ethers observed by Vanhove et al. [199] during the pre-ignition period of 1-hexene in a rapid compression machine.

Figure 20: Internal addition/decomposition of 1-penten-3yl-peroxy radicals extrapolated from the mechanism proposed by Lodhi and Walker [183].

Figure 21: Low temperature mechanism oxidation of cycloalkanes as proposed by the team of Milano for cyclohexane [227].

Figure 22: Comparison between the validation of the models of Livermore [224], Milano [227] and Nancy [228] for the oxidation of cyclohexane in a rapid compression machine ( $\mathrm{P}_{\mathrm{c}}$ from 7 to 9 bar, $\phi=1$ [229]) for autoignition and cool flame delay times vs. temperature. Symbols correspond to experiments and lines to simulations. 
Figure 23: Comparison between the validation of the models of Livermore [224], Milano [227] and Nancy [228] for the oxidation of cyclohexane in a rapid compression machine ( $\mathrm{P}_{\mathrm{c}}$ from 7 to 9 bar, $\phi=1$ [229]) for the formation of oxygenated products at $722 \mathrm{~K}$. Symbols correspond to experiments and lines to simulations.

Figure 24: Internal isomerization of peroxycyclohexanyl radicals involving a 7 membered transition state ring.

Figure 25: Mechanism for the oxidation of benzene and toluene as proposed by Brezinsky [237].

Figure 26: Cyclic ethers detected by Roubaud et al. [244] during the oxidation of n-butylbenzene in a rapid compression machine prior autoignition $\left(\mathrm{T}_{\mathrm{c}}=691 \mathrm{~K}\right)$.

Figure 27: Comparison between the experimental [271-272] (symbols) and simulated [270] (lines) conversions of the three isomers of xylene in a flow reactor $(\mathrm{T}=1155 \mathrm{~K}, \mathrm{P}=$ 1 atm and $\phi$ close to 1$)$.

Figure 28: Primary mechanism of the oxidation of 1-naphthalene as proposed by Bounaceur et al. $[10]$.

Figure 29: Structure of some species involved in the oxidation of benzene: (a) cyclopentadienone, (b) ortho-benzoquinone and (c) dihydrofulvalene.

Figure 30: Possible pathways leading to o-benzoquinone and cyclopentadienone from phenyperoxy radicals according to Sirjean et al. (numbers are relative free energies (298 K, kcal/mol) calculated at the B3LYP/6-311++G** level) [295].

Figure 31: Experimental [117] and simulated [324] pressure change $(\Delta \mathrm{p})$ curves for fuels A (26 $\%$ n-heptane, $74 \%$ toluene), B (5\% iso-octane, $21 \%$ n-heptane, $74 \%$ toluene) and C (10\% iso-octane, $16 \%$ n-heptane, $74 \%$ toluene) in the rapid compression machine of Cambridge (USA) $\left(\mathrm{T}_{\mathrm{c}}=827 \mathrm{~K}, \mathrm{P}_{\mathrm{c}}=41.6\right.$ bar, $\left.\phi=0.4\right)$. 
Figure 32: Examples of "cross term" reactions during the oxidation of n-decane/1-methylnaphthalene mixture: (a) H-abstractions by phenylbenzyl radicals from n-decane (the 5 isomers of decyl radicals can be obtained), (b) combinations between phenylbenzyl and decyl radicals [10]. 
Figure 1
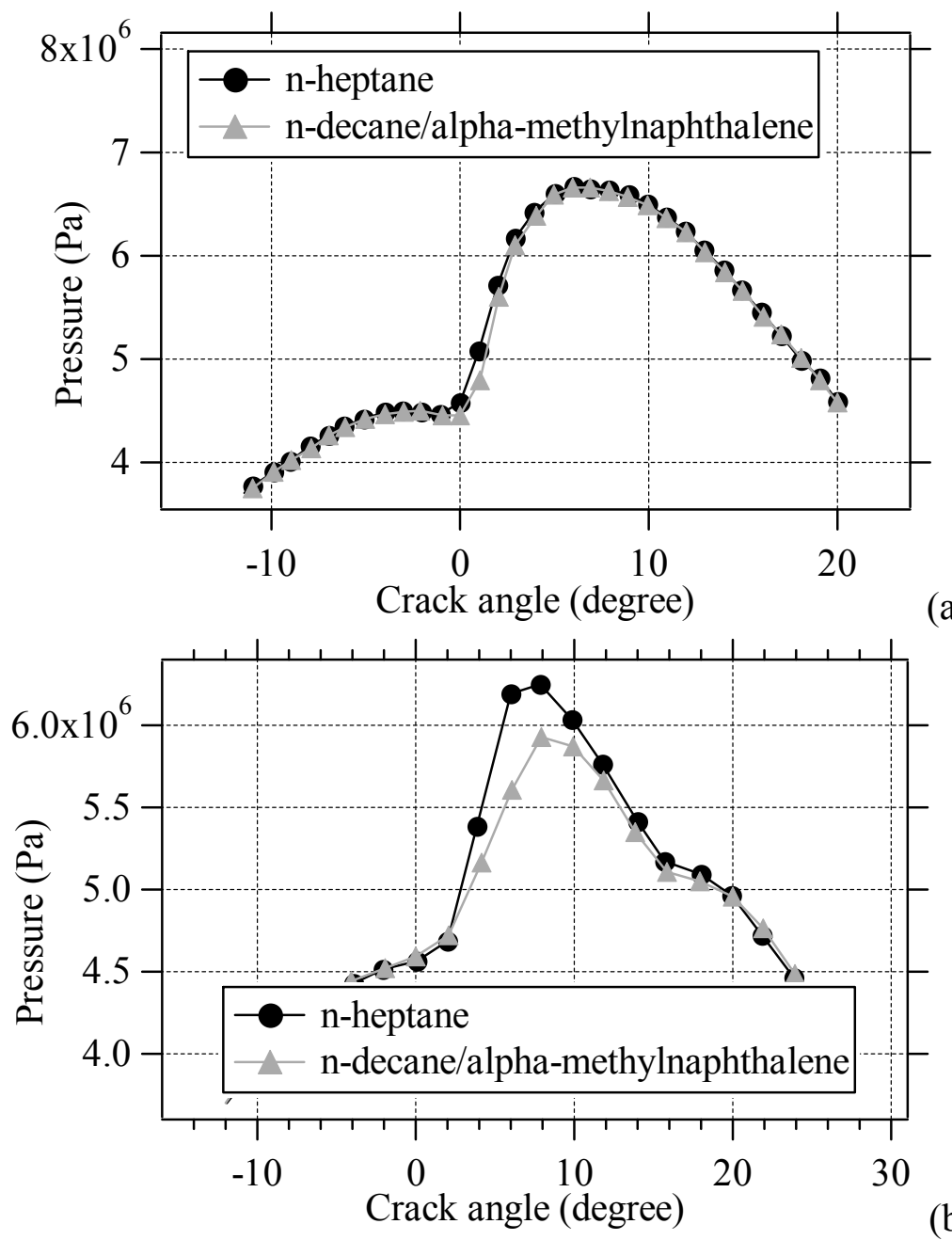

Figure 1: Simulated influence of the fuel composition (n-heptane or n-decane/ $\alpha$-methylnaphthalene mixture) on the simulated pressure evolutions in (a) a low load direct injection diesel engine and in (b) a split injection HCCI engine [10]. 


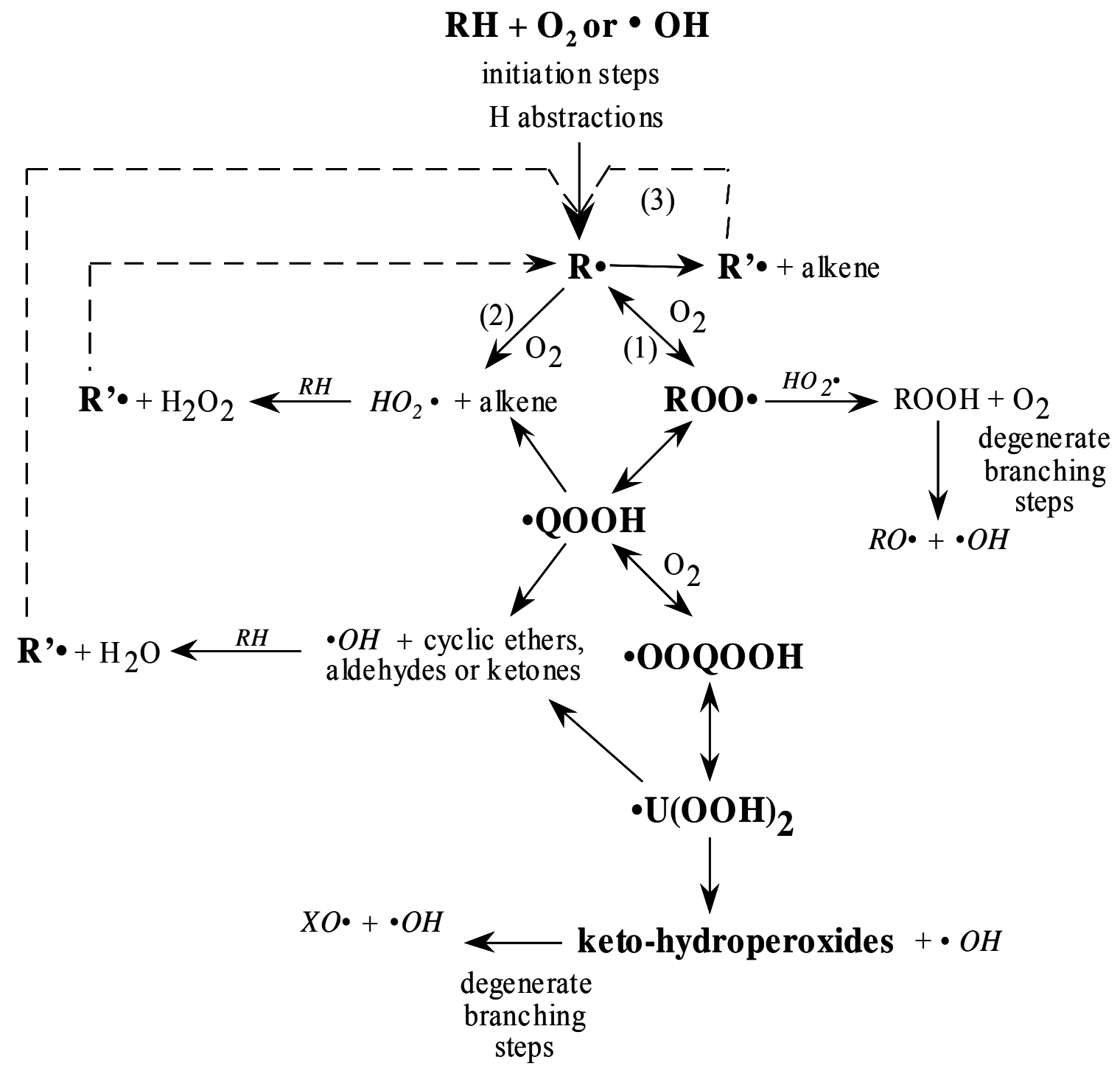

Figure 2: Simplified scheme for the primary mechanism of oxidation of alkanes. 
Figure 3

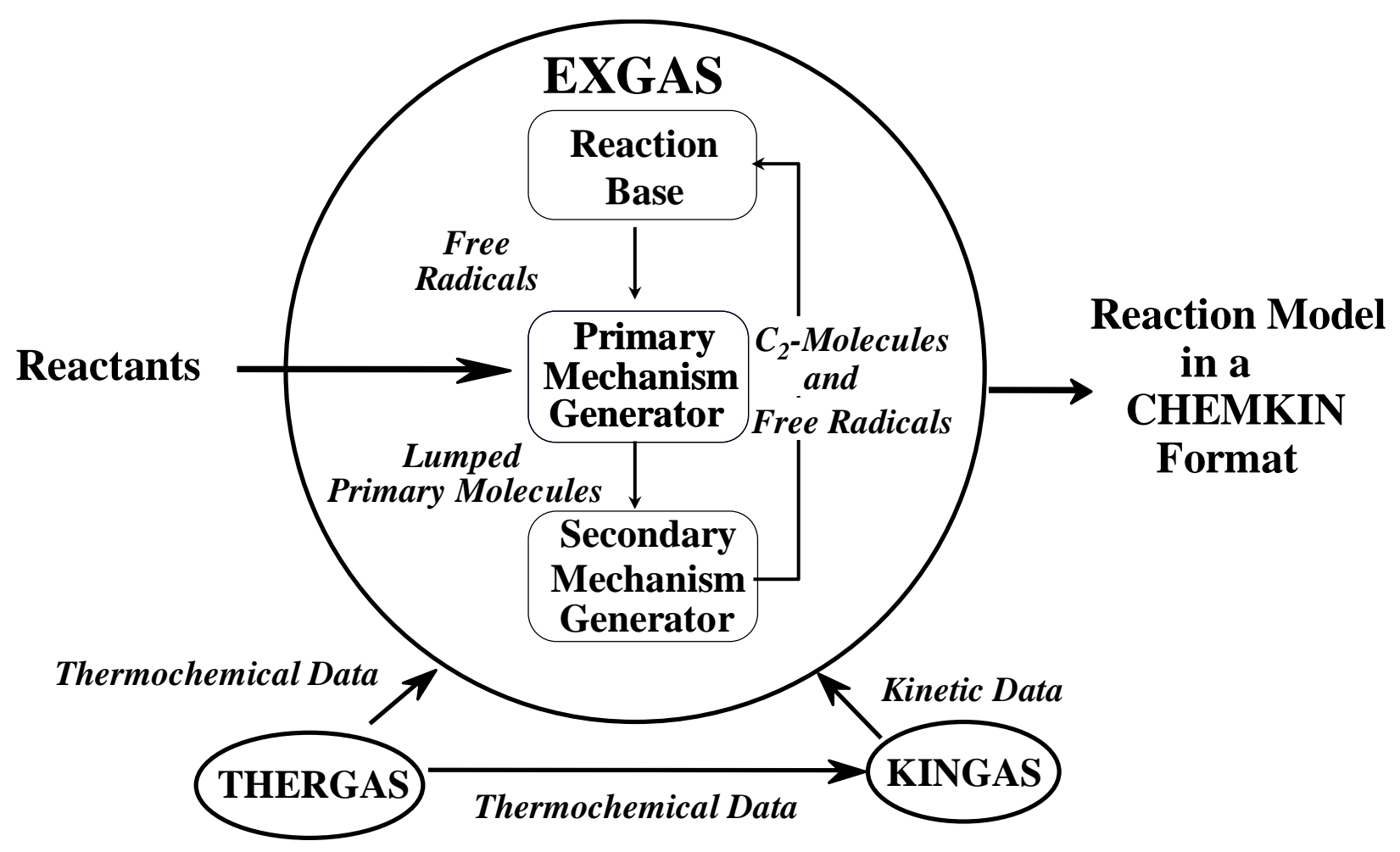

Figure 3: Schematic structure of EXGAS software. 
Figure 4
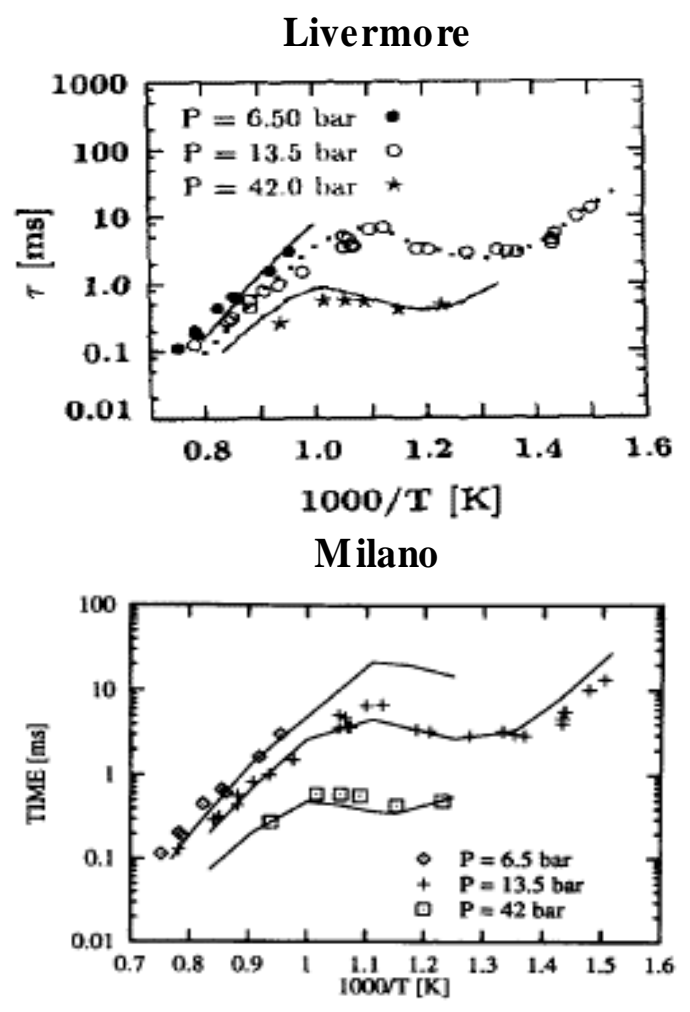

Nancy

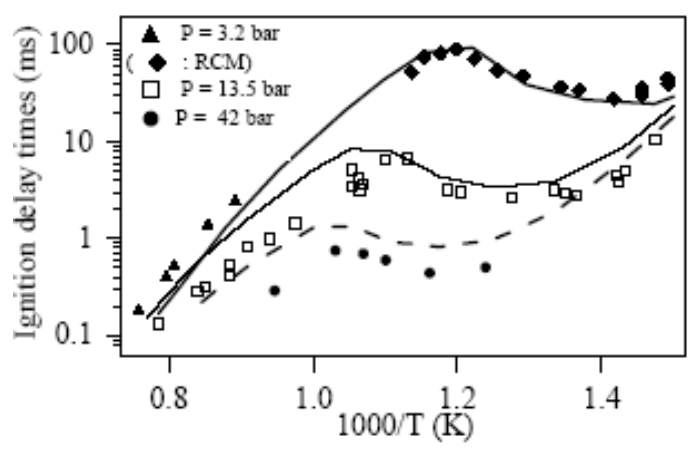

Figure 4: Comparison between the validation of the models of Livermore [70], Milano [96] and Nancy [24] for n-heptane in a shock tube $(\phi=1$ [118], rapid compression machine data at $\mathrm{P}_{\mathrm{c}}$ from 3 to 4 bar [116] are also shown on the graph of Nancy). Symbols correspond to experiments and lines to simulations. 
Figure 5

\section{Livermore}

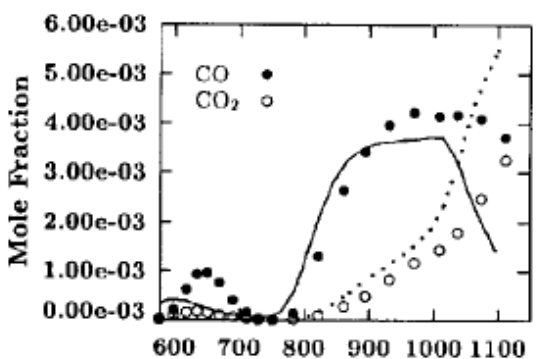

Milano

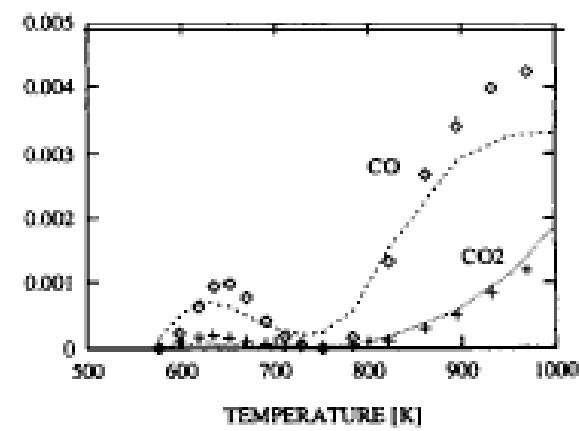

Nancy

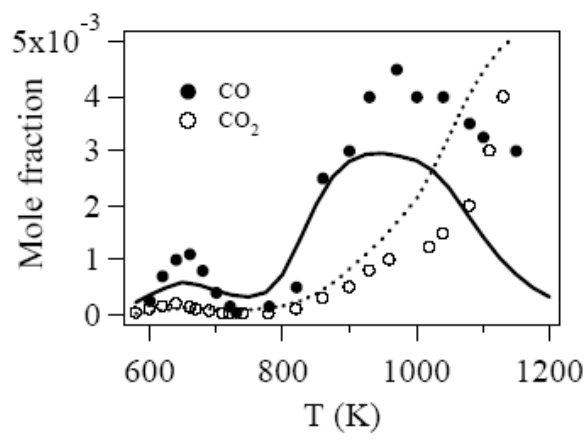

Figure 5: Comparison between the validation of the models of Livermore [70], Milano [96] and Nancy [24] for $n$-heptane in a jet-stirred reactor $(0.1 \% \mathrm{n}$-heptane, $10 \mathrm{~atm}, \phi=1, \tau=1 \mathrm{~s}$ [132]). Symbols correspond to experiments and lines to simulations. 
Figure 6

\section{Livermore}

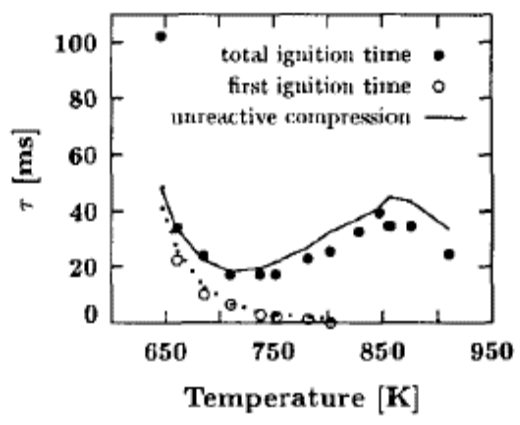

Nancy

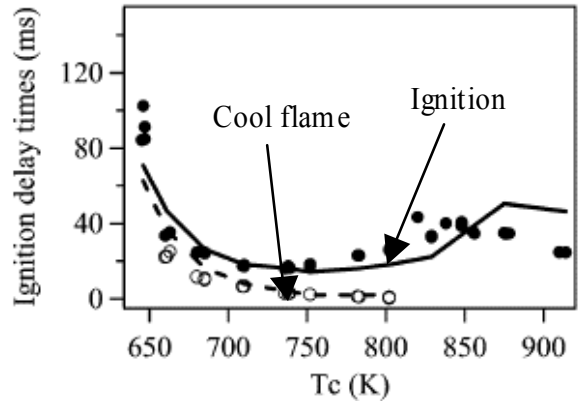

Figure 6: Comparison between the validation of the models of Livermore [70] and Nancy [24] for $n$-heptane in a rapid compression machine ( $\mathrm{P}_{\mathrm{c}}$ from 3.7 bar to 4.6 bar , $\phi=1$ [116]).

Symbols correspond to experiments and lines to simulations. 
Figure 7

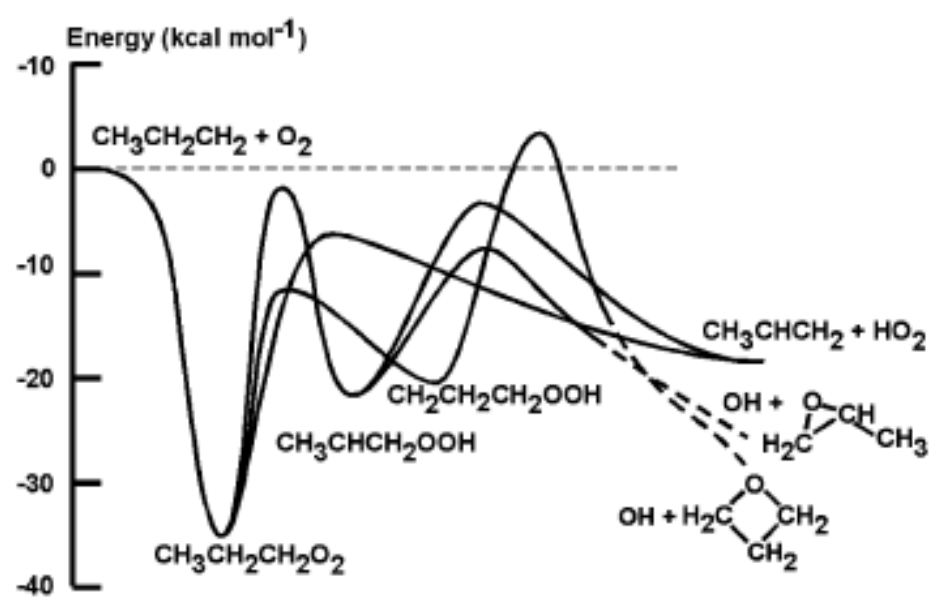

Figure 7: Schematic potential energy surfaces for the reactions of n-propyl radicals with oxygen molecules according to DeSain et al. [159]. 
Figure 8

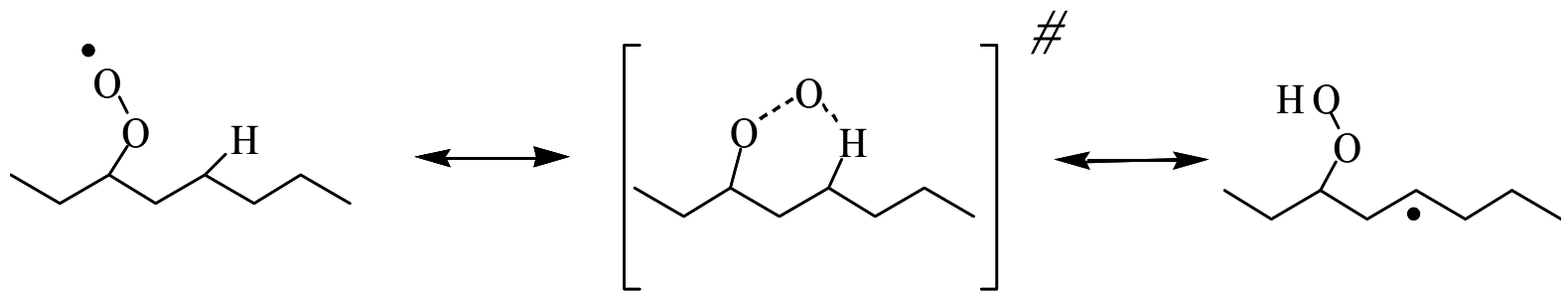

Figure 8: Isomerization by internal transfer of $\mathrm{H}$-atom from 2-heptylperoxy radicals. 
Figure 9

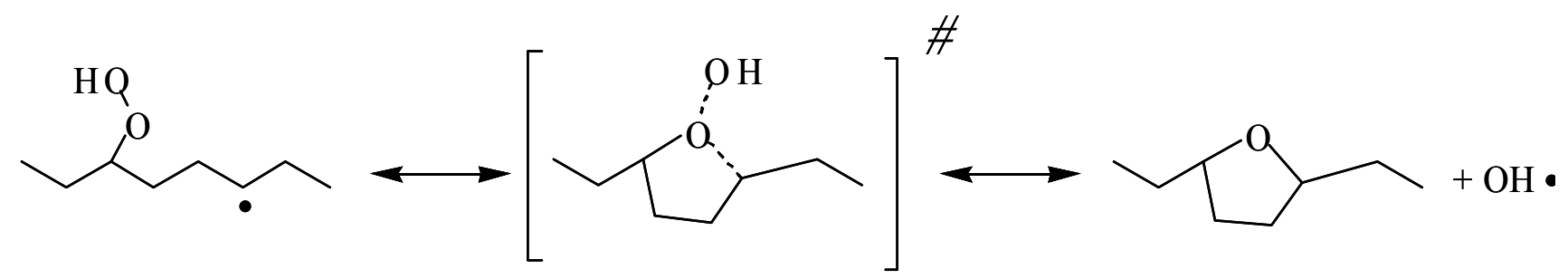

Figure 9: Formation of a cyclic ether from 2-hydroperoxy-5-heptyl radicals. 
Breaking of a C-O bond

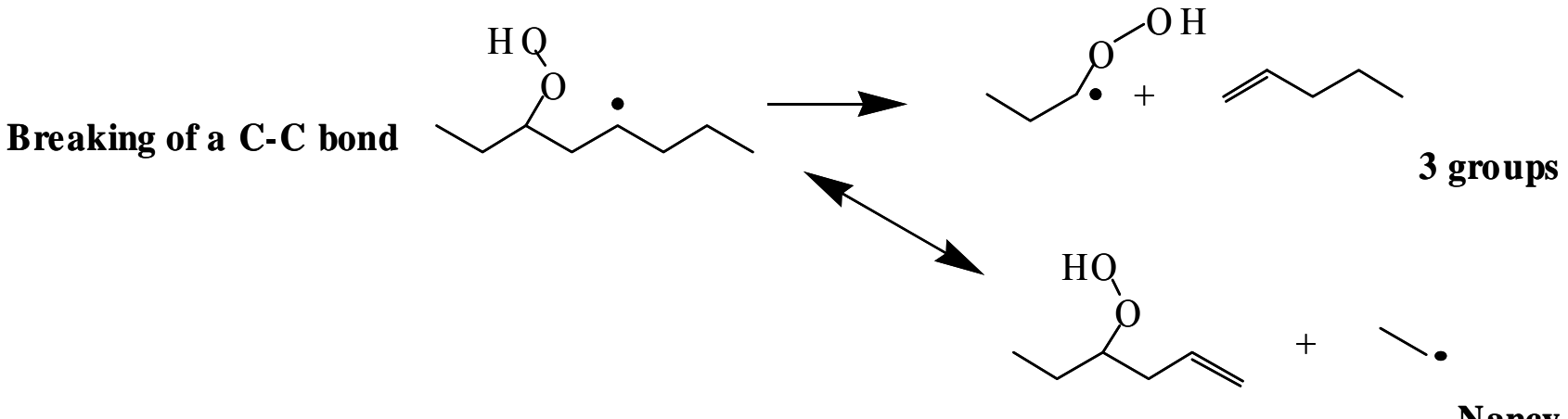

Breaking of a 0-O bond

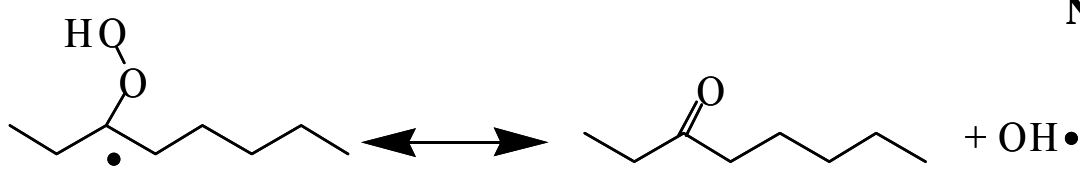

Nancy

Figure 10: Examples of decompositions of hydroperoxy octyl radicals. 
Figure 11

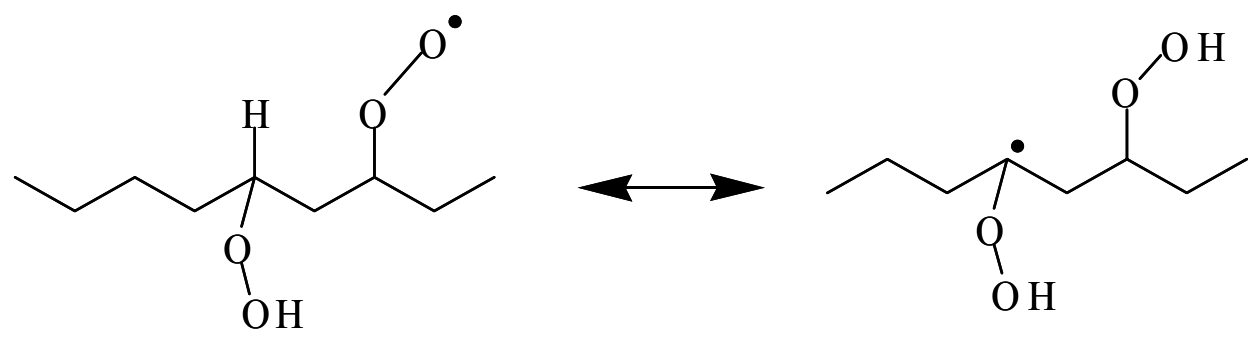

Figure 11: Example of an isomerization involving the transfer of an $\mathrm{H}$-atom bound to a carbon atom bound to an oxygen atom. 

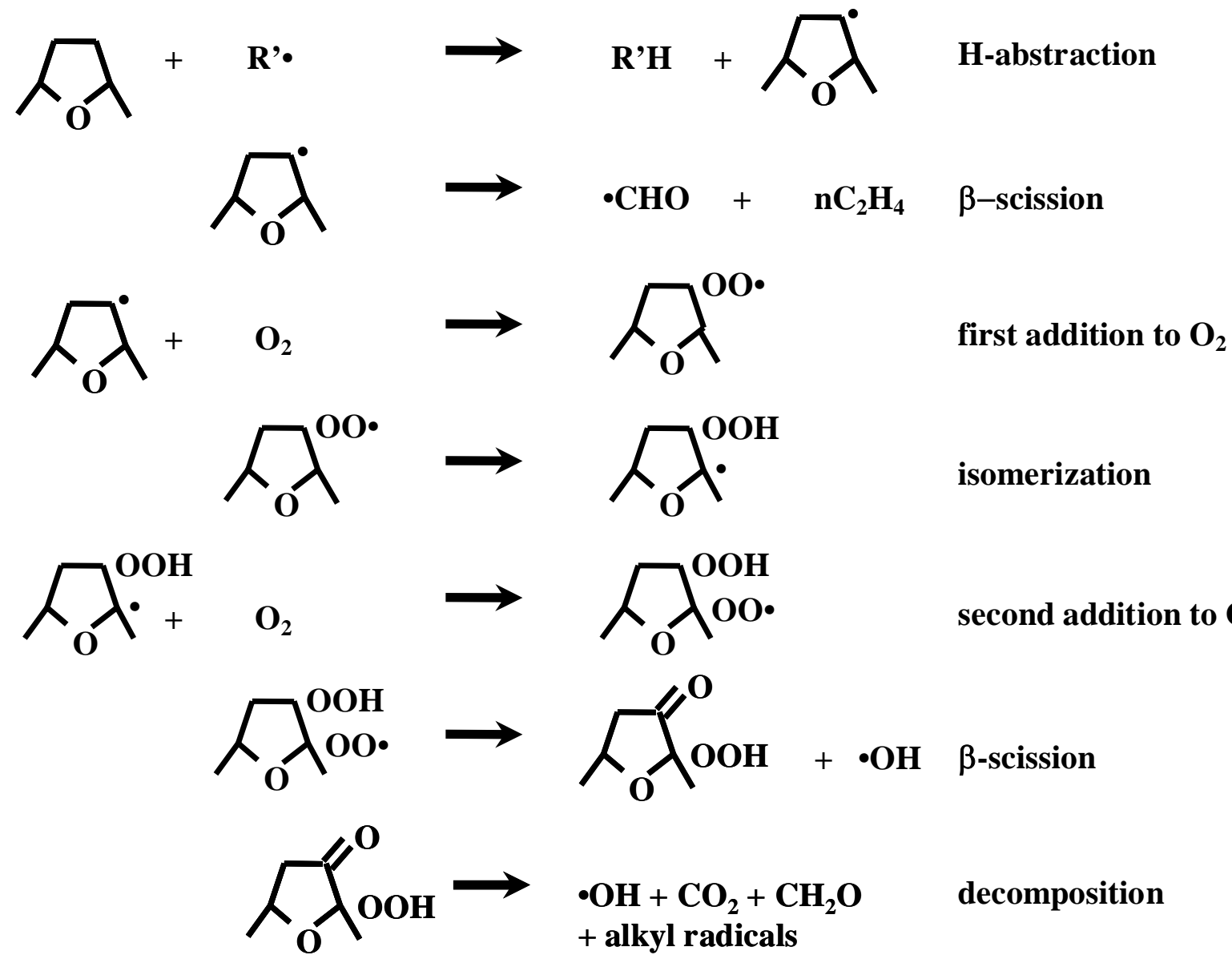

Figure 12: Secondary reactions considered in the mechanism of Nancy for a cyclic ether deriving from n-heptane [162]. 
Figure 13

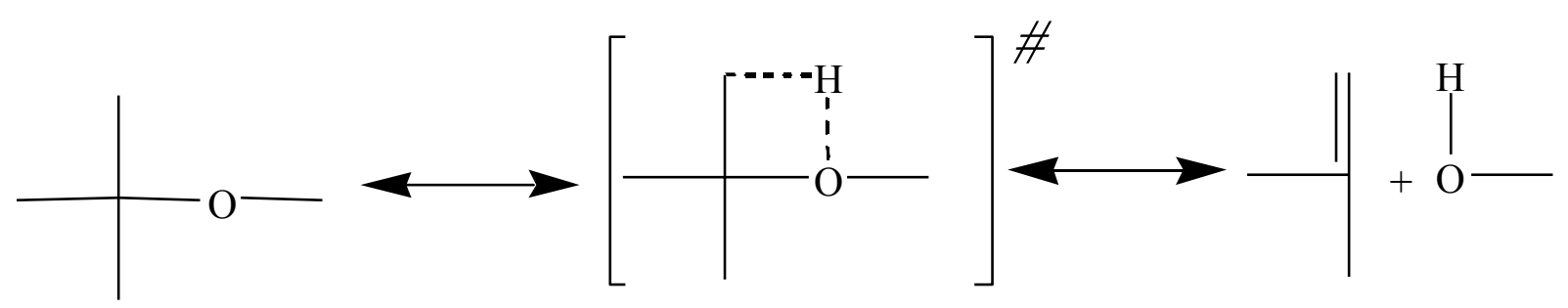

(a)

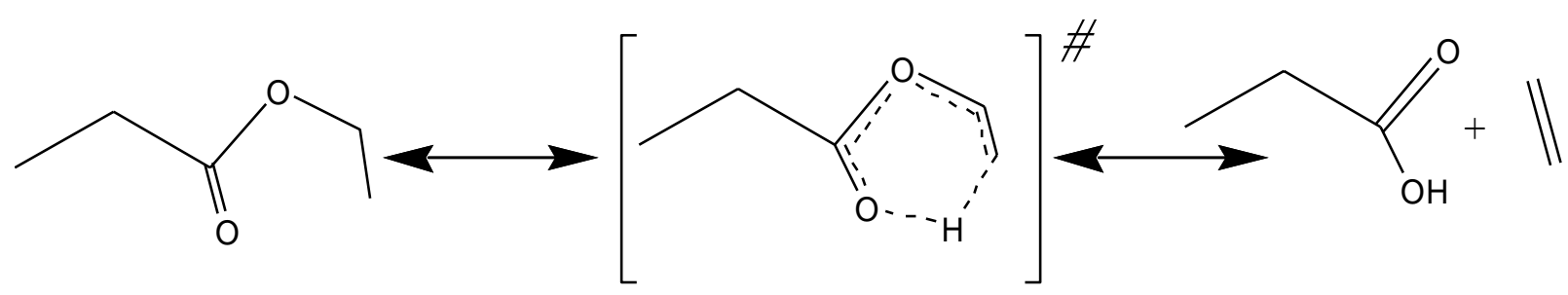

(b)

Figure 13: Examples of molecular reaction in the case of (a) MTBE and (b) ethyl propanoate. 
Figure 14
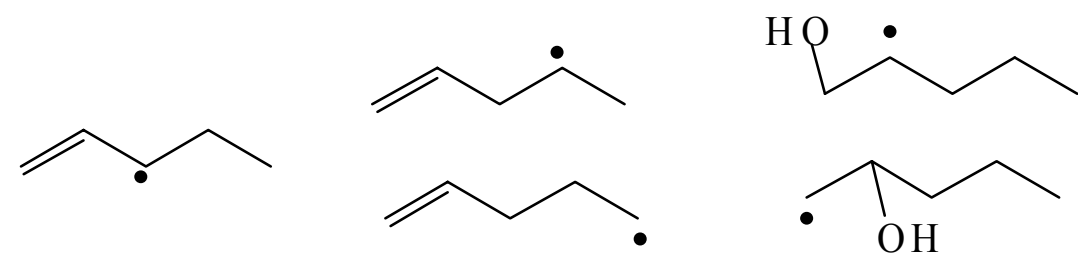
Allylic alkenyl radicals
Alkylic alkenyl radicals

Figure 14: Examples of alkenyl and hydroxyalkyl radicals illustrated in the case of 1-pentene. 


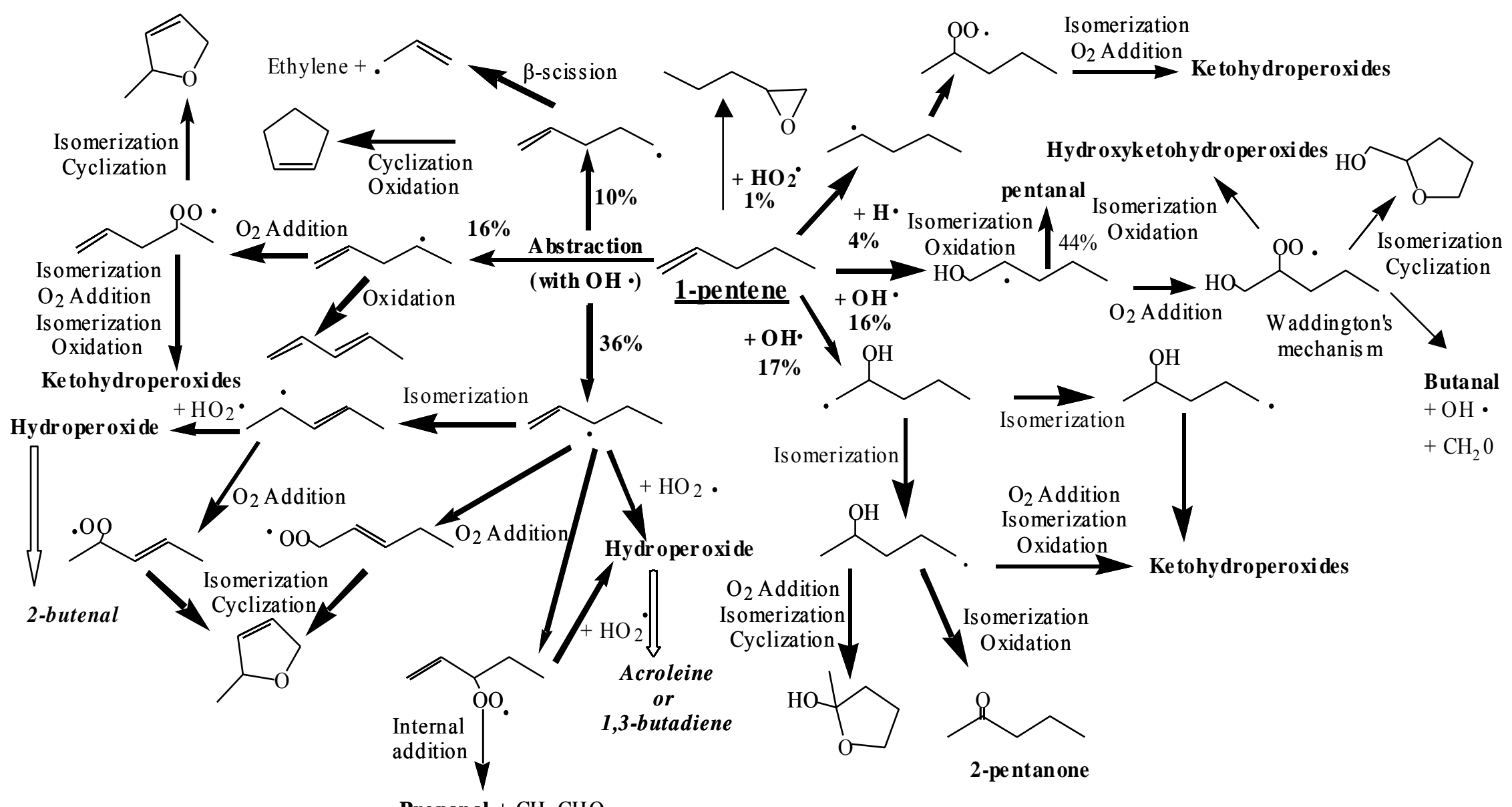

Figure 15: Low temperature mechanism oxidation of alkenes exemplified by a rate of production analysis of the oxidation of a stoichiometric 1-pentene/air mixture at $800 \mathrm{~K}$ and 7.5 bar [186]. 
Figure 16

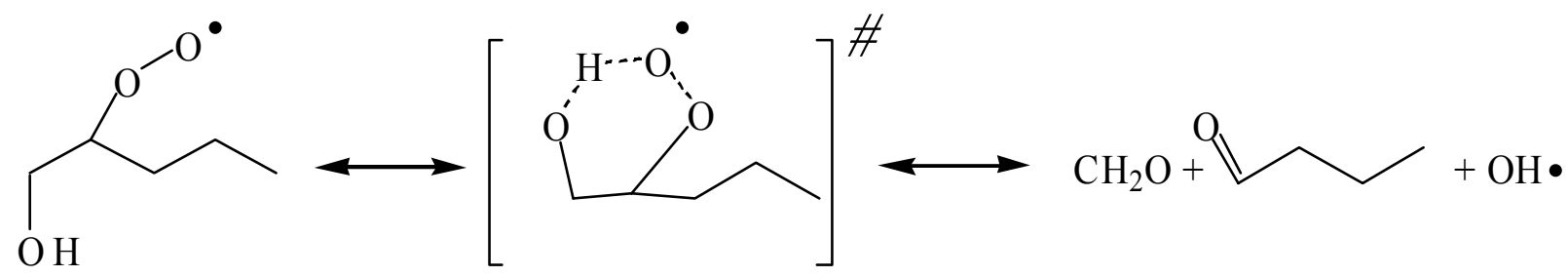

Figure 16: Isomerization of hydroxypentylperoxy radicals according to the mechanism of Waddington [190]. 

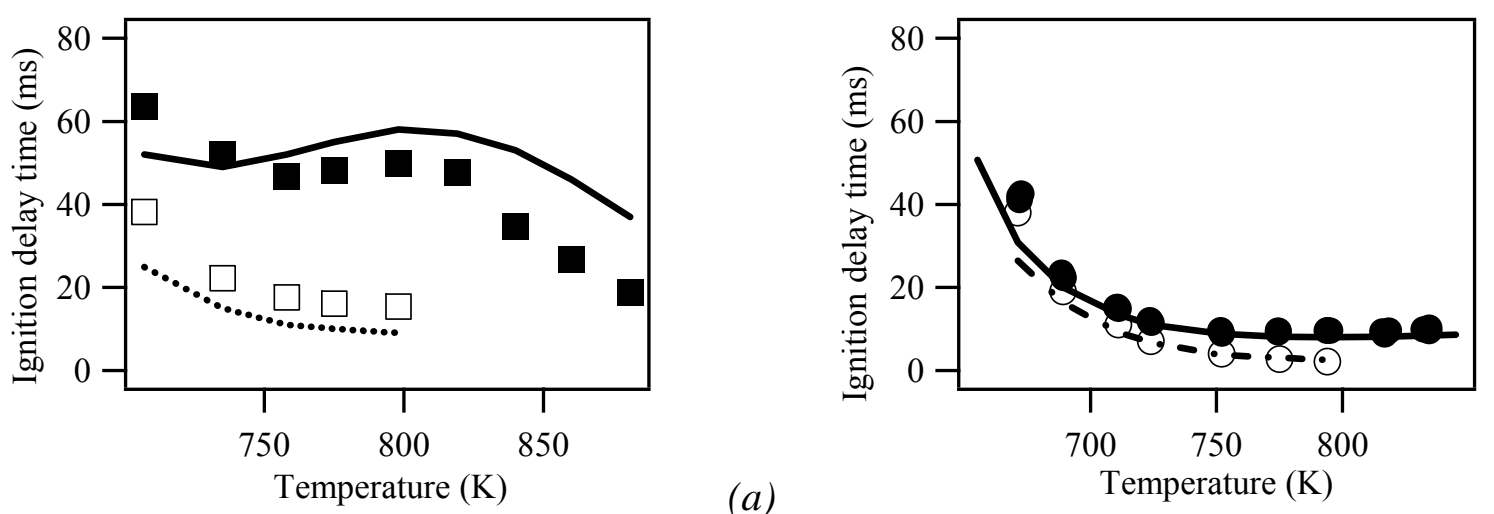

(b)

Figure 17: Validation of the models Nancy [186] for (a) 1-pentene and (b) 1-hexene using results obtained in the rapid compression machine of Lille ( $\mathrm{P}_{\mathrm{c}}$ from 3.7 bar to 4.6 bar , $\left.\phi=1[197,199]\right)$. Symbols (white dots for cool flame and black ones for autoignition) correspond to experiments and lines to simulations. 
Figure 18

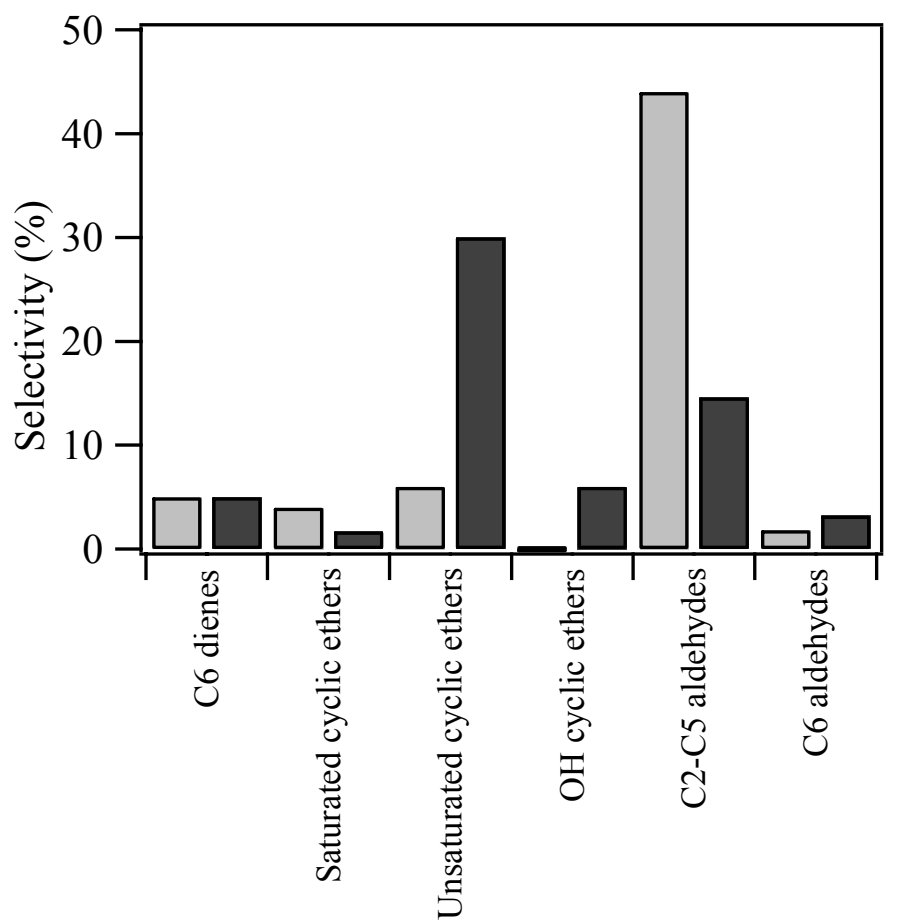

Figure 18: Comparison between the experimental (light grey) [198] and the predicted (dark grey) [203] selectivity of products during the pre-ignition period of 1-hexene under the conditions of figure $17 \mathrm{~b}\left(\mathrm{~T}_{\mathrm{c}}=707 \mathrm{~K}\right)$. 
Figure 19
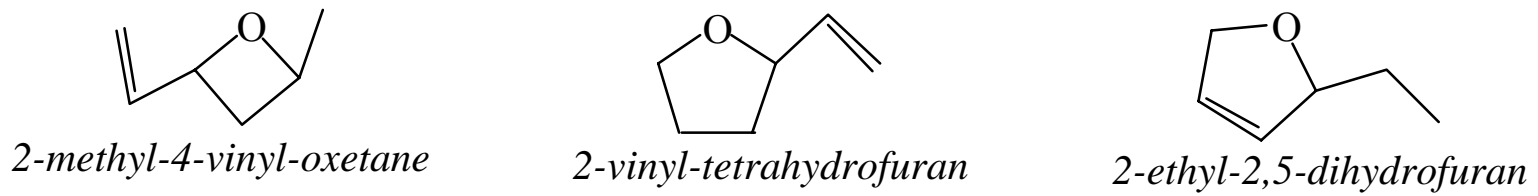

Figure 19: Unsaturated cyclic ethers observed by Vanhove et al. [199] during the pre-ignition period of 1-hexene in a rapid compression machine. 
Figure 20

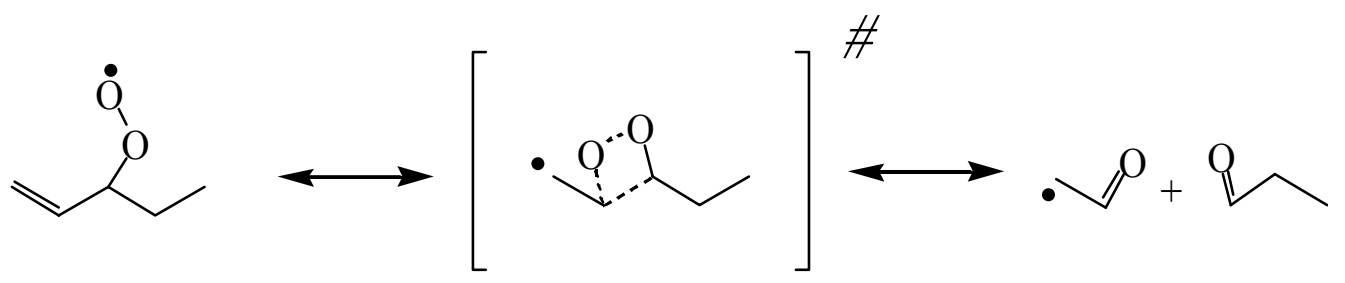

Figure 20: Internal addition/decomposition of 1-penten-3yl-peroxy radicals extrapolated from the mechanism proposed by Lodhi and Walker [183]. 
Figure 21

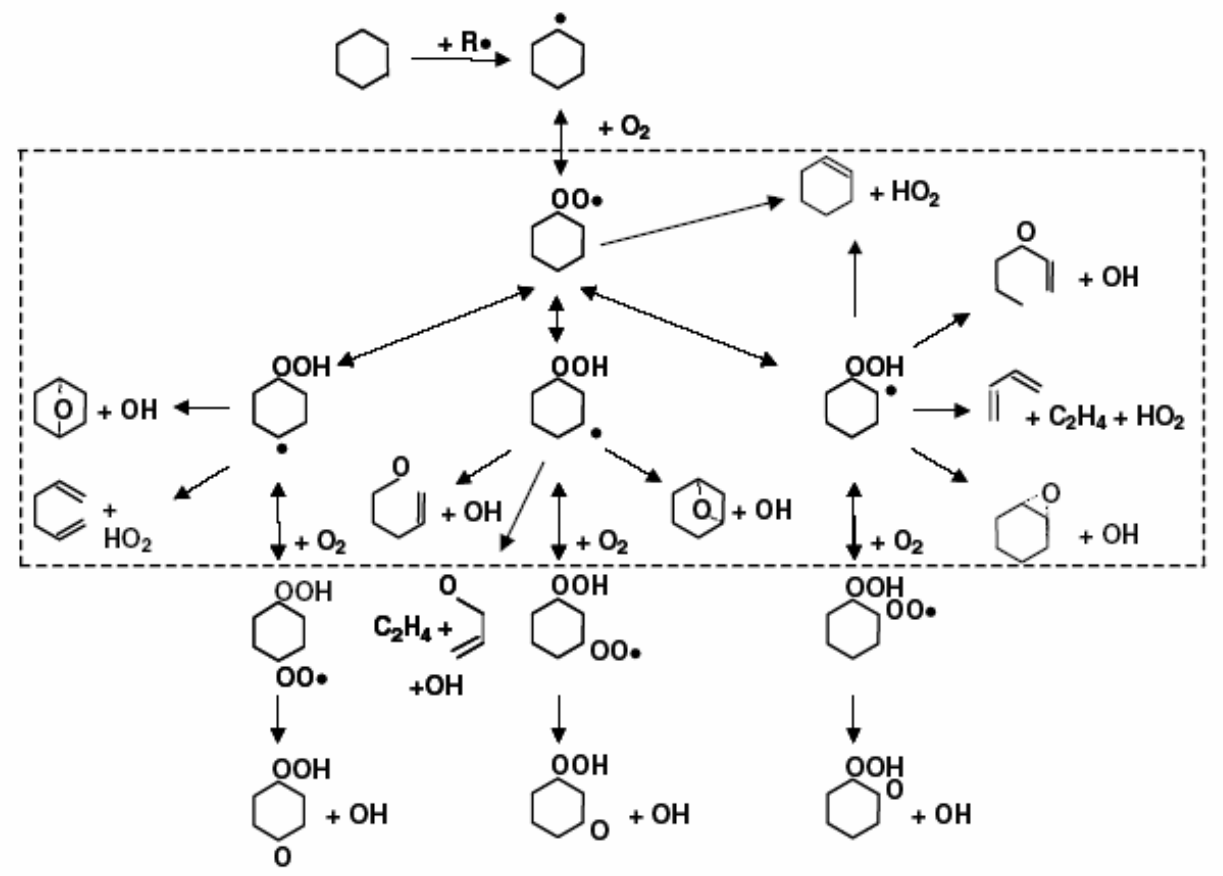

Figure 21: Low temperature mechanism oxidation of cycloalkanes as proposed by the team of Milano for cyclohexane [227]. 
Figure 22

\section{Livermore}

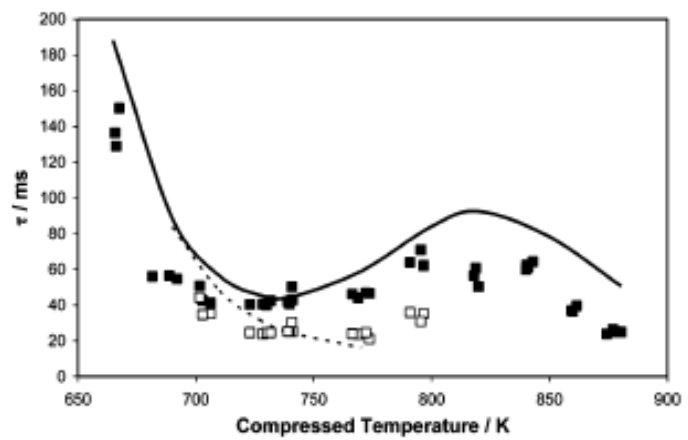

Milano

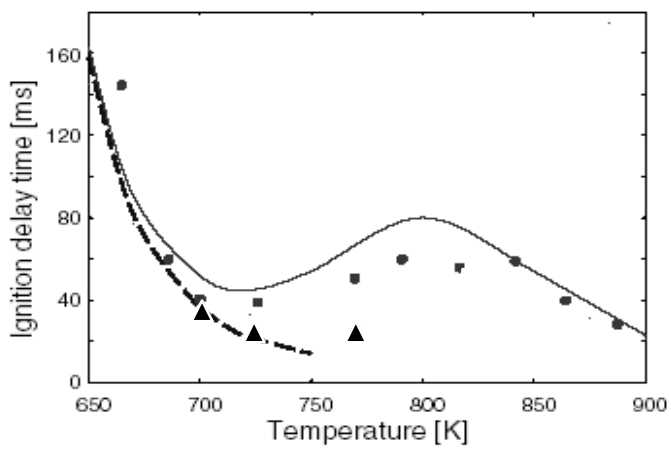

Nancy

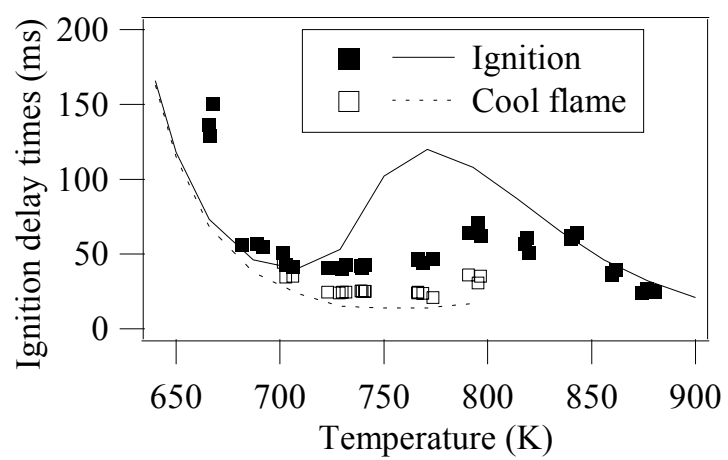

Figure 22: Comparison between the validation of the models of Livermore [224], Milano [227] and Nancy [228] for the oxidation of cyclohexane in a rapid compression machine $\left(\mathrm{P}_{\mathrm{c}}\right.$ from 7 to

9 bar, $\phi=1$ [229]) for autoignition and cool flame delay times vs. temperature. Symbols correspond to experiments and lines to simulations. 
Figure 23
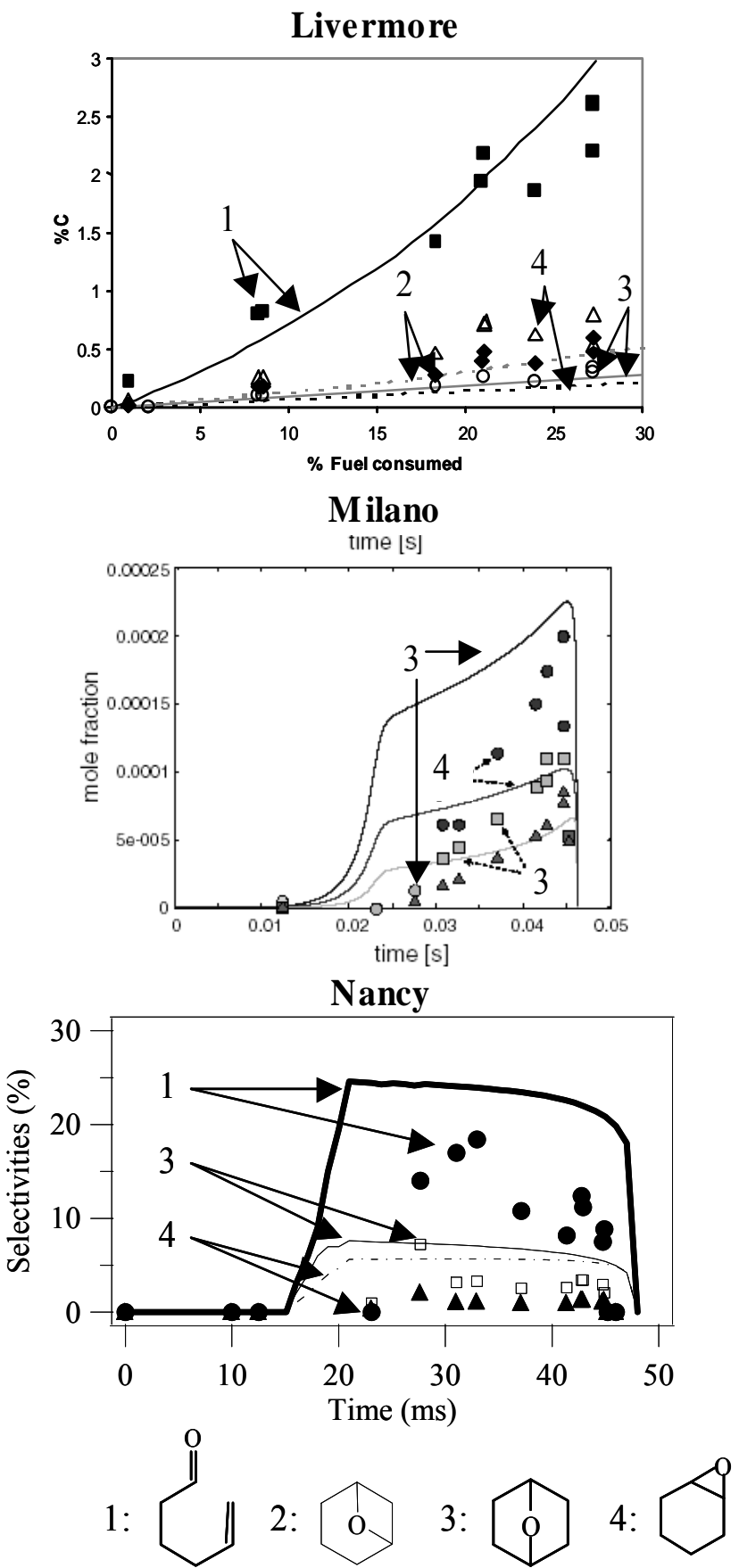

Figure 23: Comparison between the validation of the models of Livermore [224], Milano [227] and Nancy [228] for the oxidation of cyclohexane in a rapid compression machine $\left(\mathrm{P}_{\mathrm{c}}\right.$ from 7 to 9 bar, $\phi=1$ [229]) for the formation of oxygenated products at $722 \mathrm{~K}$. Symbols correspond to experiments and lines to simulations. 
Figure 24
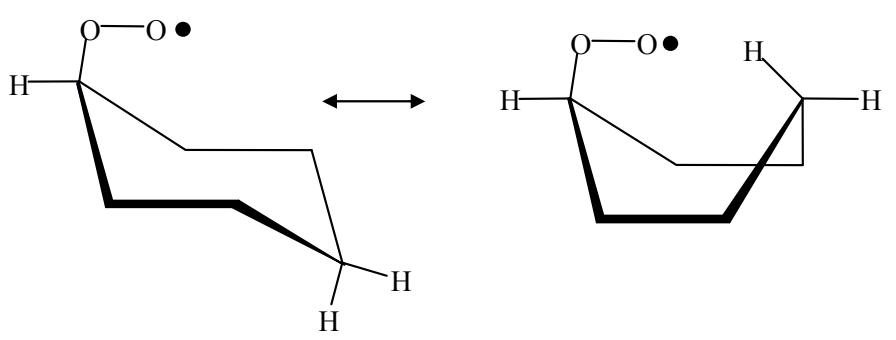

Chair form

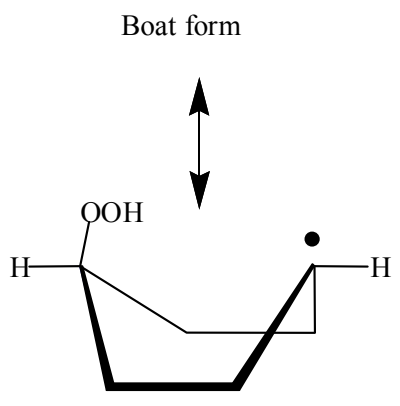

Figure 24: Internal isomerization of peroxycyclohexanyl radicals involving a 7 membered transition state ring. 


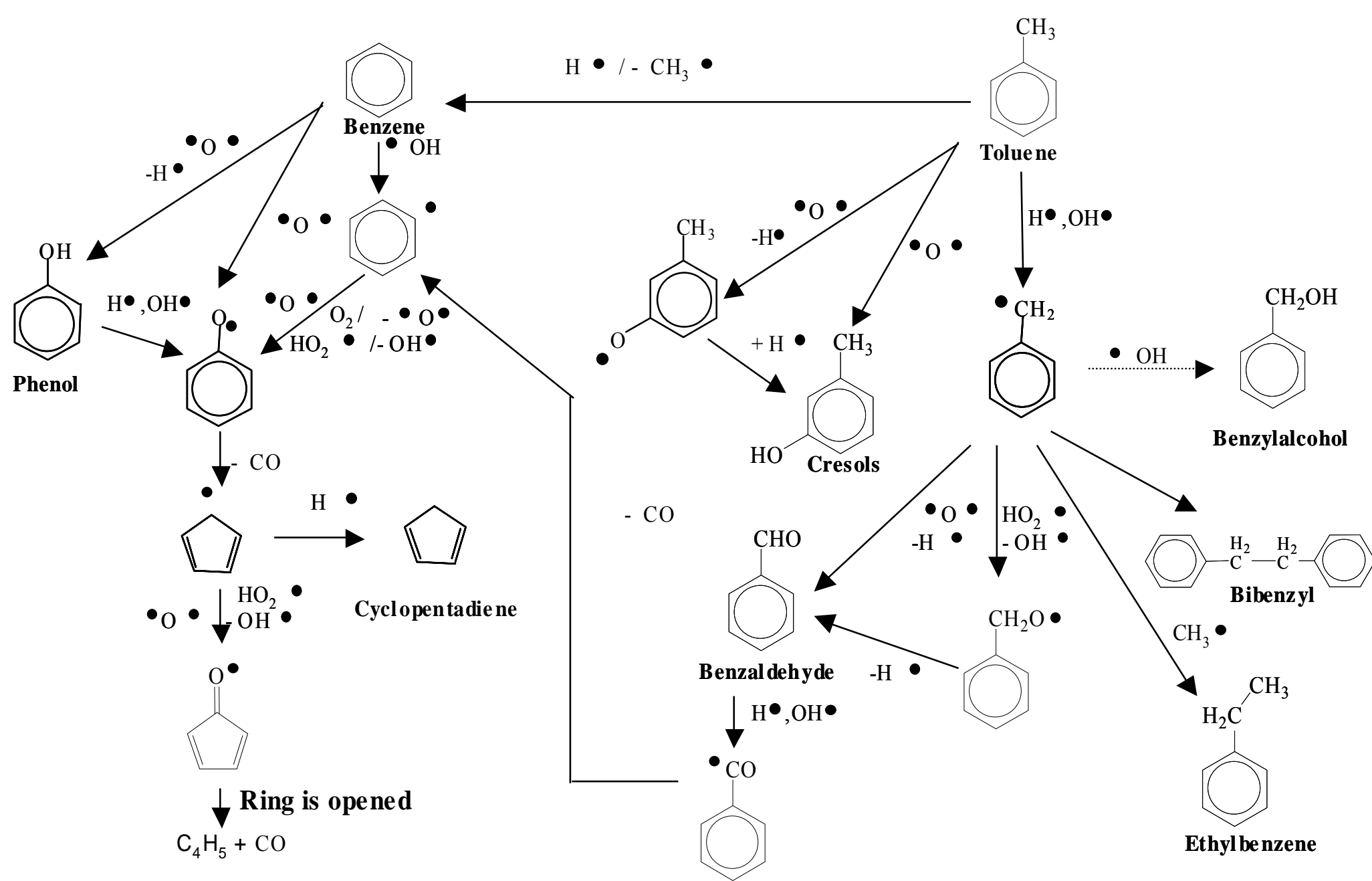

Mechanism of benzene

Mechanism of toluene

Figure 25: Mechanism for the oxidation of benzene and toluene as proposed by Brezinsky [237]. 
Figure 26<smiles>CC1CC(c2ccccc2)O1</smiles>

2-methyl-4-phenyloxetane<smiles>CCC1OC1c1ccccc1</smiles>

2-ethyl-3-phenyloxirane<smiles>c1ccc(CC2CCO2)cc1</smiles>

2-benzyloxetane<smiles>CC1OC1Cc1ccccc1</smiles>

2-benzyl-3-methyloxirane<smiles>c1ccc(C2CCCO2)cc1</smiles>

2-phenyltetrahydrofuran

Figure 26: Cyclic ethers detected by Roubaud et al. [244] during the oxidation of n-butylbenzene in a rapid compression machine prior autoignition $\left(T_{c}=691 \mathrm{~K}\right)$. 
Figure 27

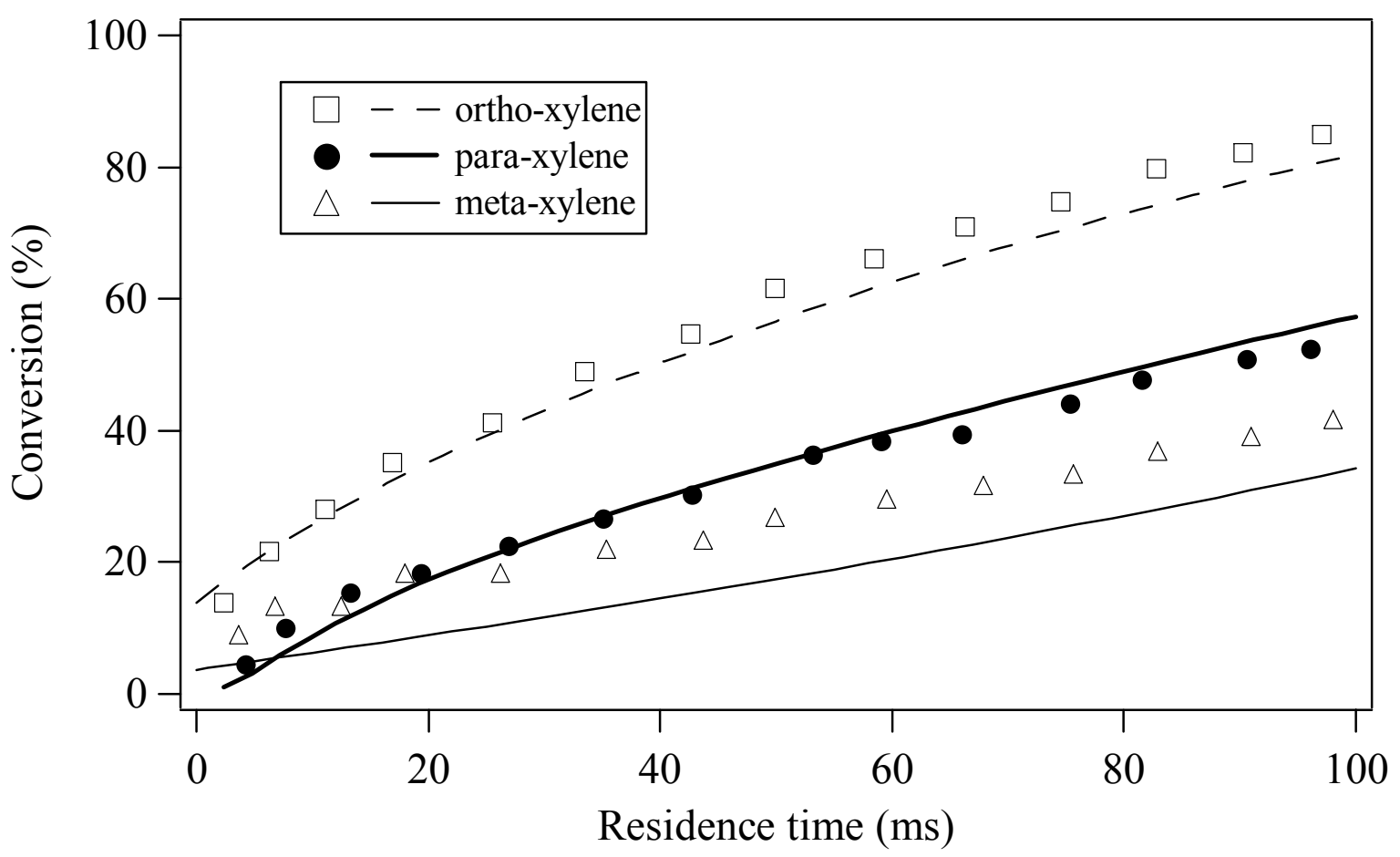

Figure 27: Comparison between the experimental [271-272] (symbols) and simulated [270] (lines) conversions of the three isomers of xylene in a flow reactor $(\mathrm{T}=1155 \mathrm{~K}, \mathrm{P}=1$ atm and $\phi$ close to 1$)$. 


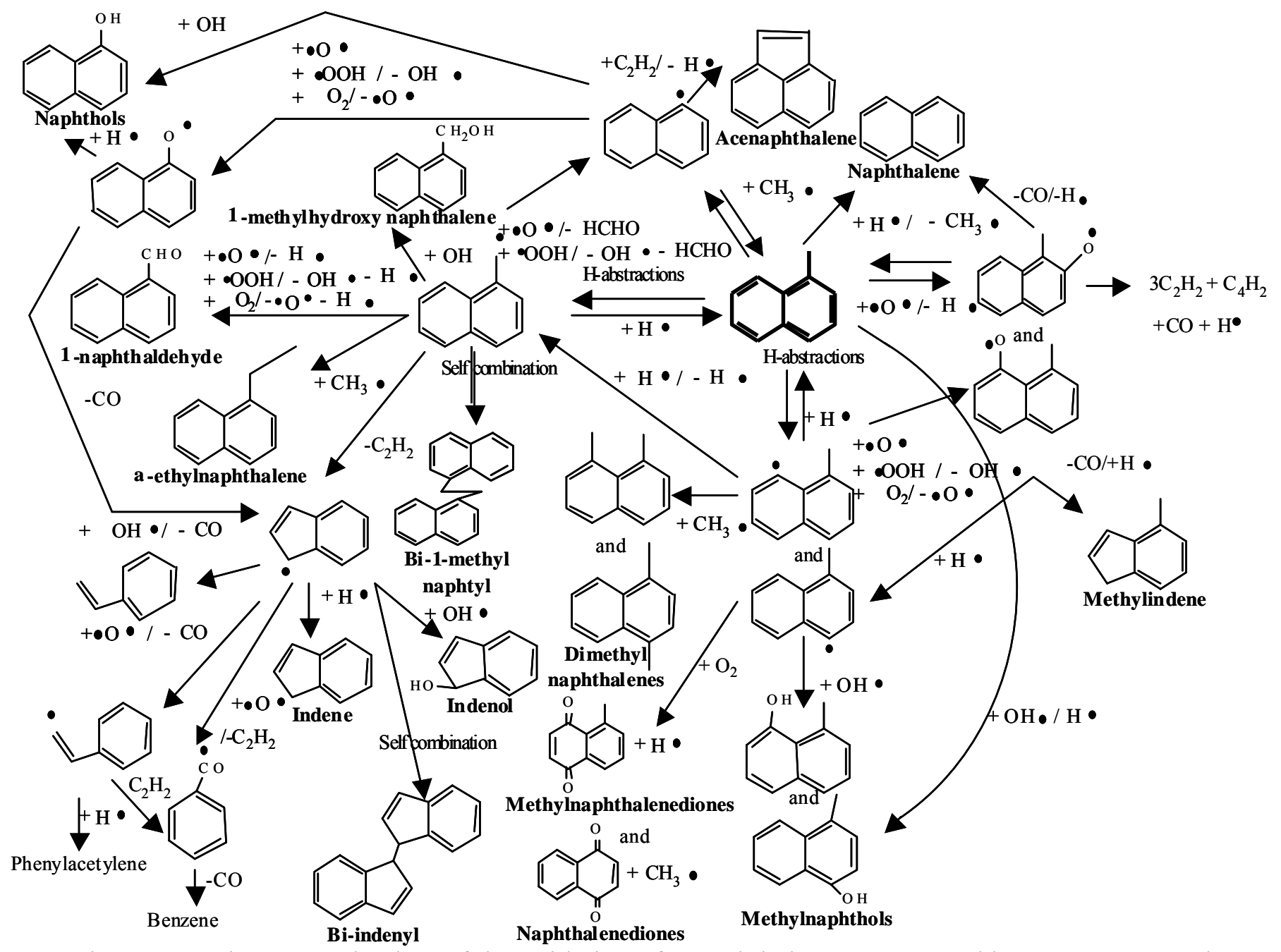

Figure 28: Primary mechanism of the oxidation of 1-naphthalene as proposed by Bounaceur et al. [10]. 
Figure 29
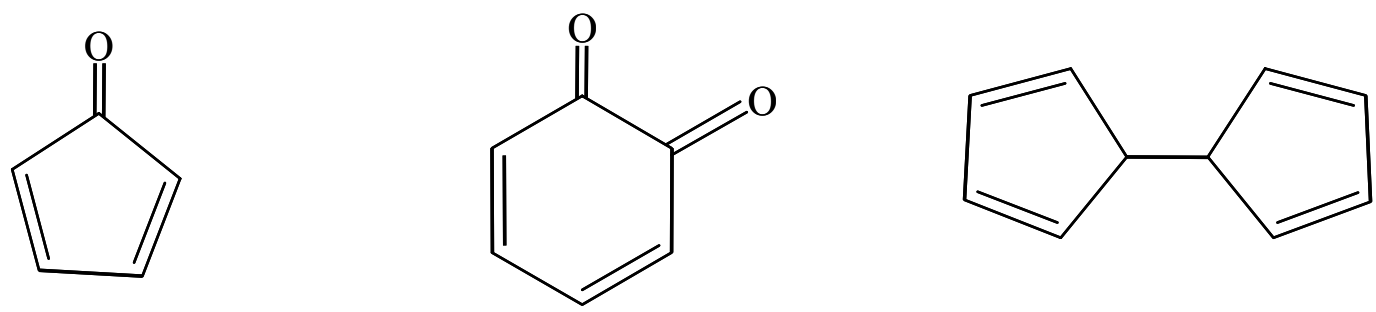

(a)

(b)

(c)

Figure 29: Structure of some species involved in the oxidation of benzene: (a) cyclopentadienone, (b) ortho-benzoquinone and (c) dihydrofulvalene. 
Figure 30

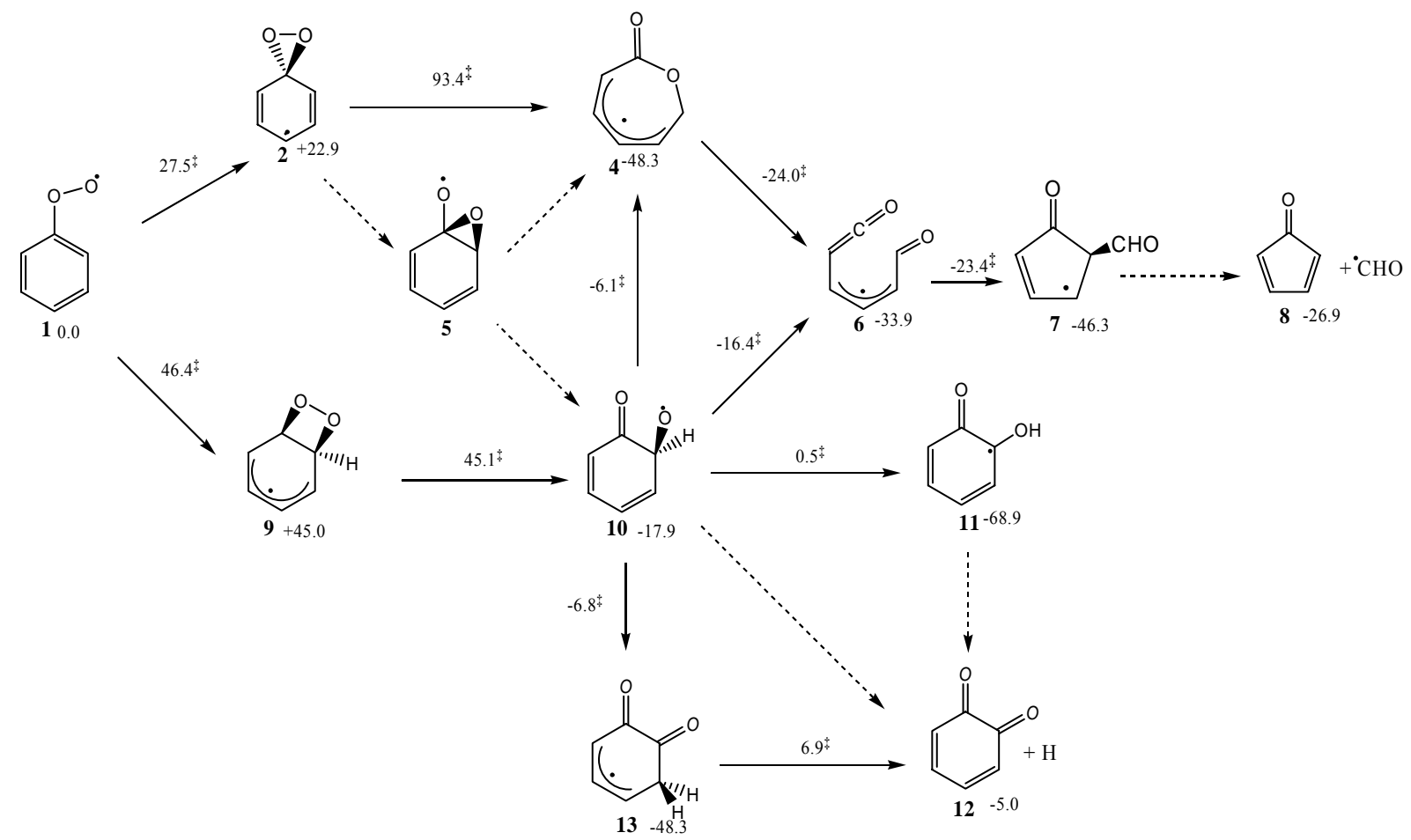

Figure 30: Possible pathways leading to o-benzoquinone and cyclopentadienone from phenyperoxy radicals according to Sirjean et al. (numbers are relative free energies (298 K, $\mathrm{kcal} / \mathrm{mol}$ ) calculated at the B3LYP/6-311++G** level) [295]. 
Figure 31

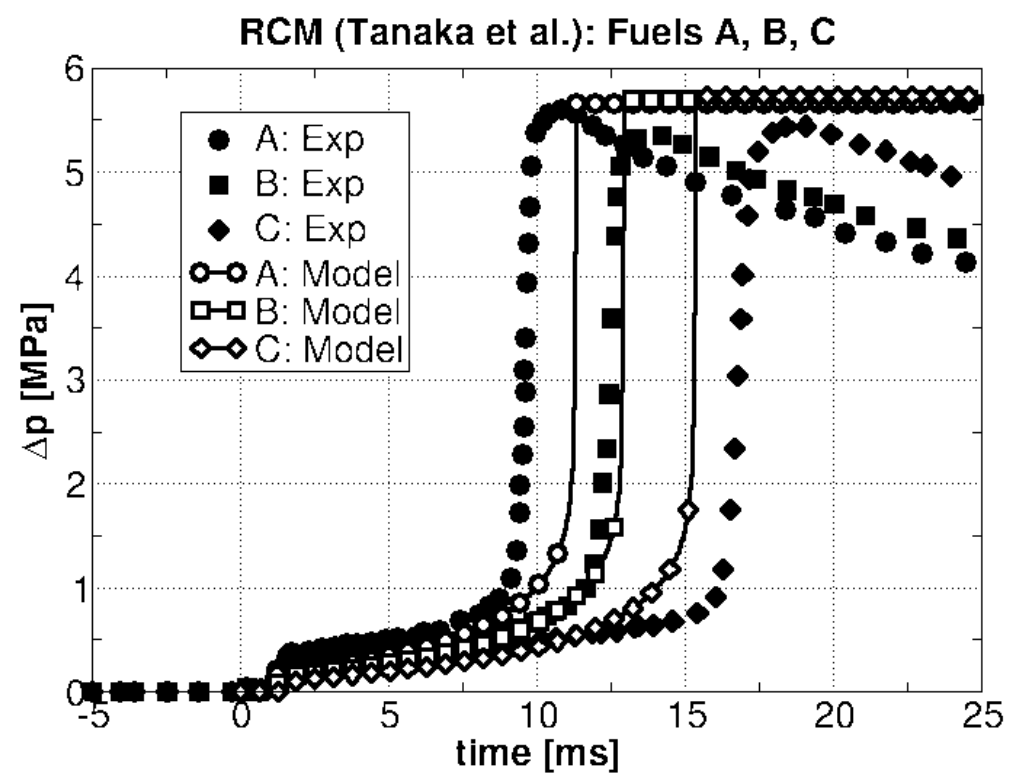

Figure 31: Experimental [117] and simulated [324] pressure change $(\Delta p)$ curves for fuels A (26 $\%$ n-heptane, $74 \%$ toluene), B (5\% iso-octane, $21 \%$ n-heptane, $74 \%$ toluene) and C (10\% iso-octane, $16 \% \mathrm{n}$-heptane, $74 \%$ toluene) in the rapid compression machine of Cambridge $(\mathrm{USA})\left(\mathrm{T}_{\mathrm{c}}=827 \mathrm{~K}, \mathrm{P}_{\mathrm{c}}=41.6 \mathrm{bar}, \phi=0.4\right)$. 
Figure 32

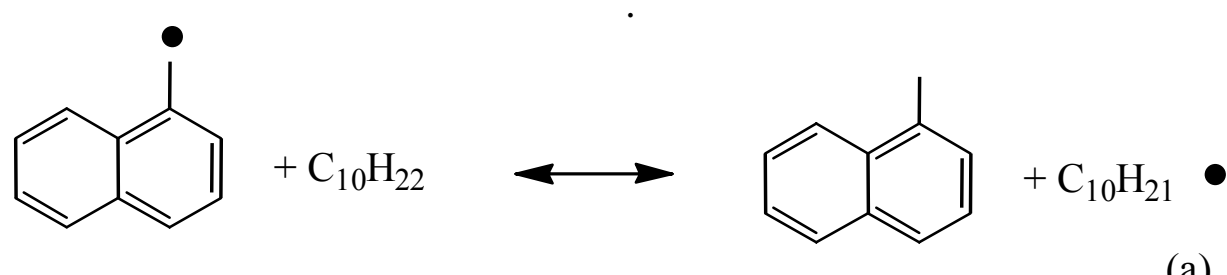

(a)<smiles>Cc1cccc2ccccc12</smiles>

(b)

Figure 32: Examples of "cross term" reactions during the oxidation of n-decane/1-methylnaphthalene mixture: (a) $\mathrm{H}$-abstractions by phenylbenzyl radicals from n-decane (the 5 isomers of decyl radicals can be obtained), (b) combinations between phenylbenzyl and decyl radicals [10]. 